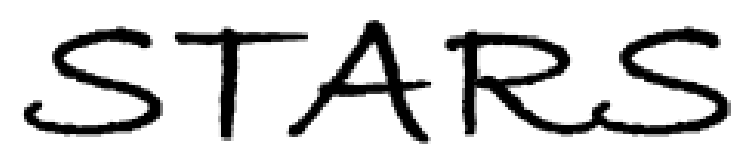

University of Central Florida

STARS

2014

\title{
Computational Fluid Dynamics Uncertainty Analysis For Payload Fairing Spacecraft Environmental Control Systems
}

Curtis Groves

University of Central Florida

Part of the Mechanical Engineering Commons

Find similar works at: https://stars.library.ucf.edu/etd

University of Central Florida Libraries http://library.ucf.edu

This Doctoral Dissertation (Open Access) is brought to you for free and open access by STARS. It has been accepted for inclusion in Electronic Theses and Dissertations, 2004-2019 by an authorized administrator of STARS. For more information, please contact STARS@ucf.edu.

\section{STARS Citation}

Groves, Curtis, "Computational Fluid Dynamics Uncertainty Analysis For Payload Fairing Spacecraft Environmental Control Systems" (2014). Electronic Theses and Dissertations, 2004-2019. 3024. https://stars.library.ucf.edu/etd/3024

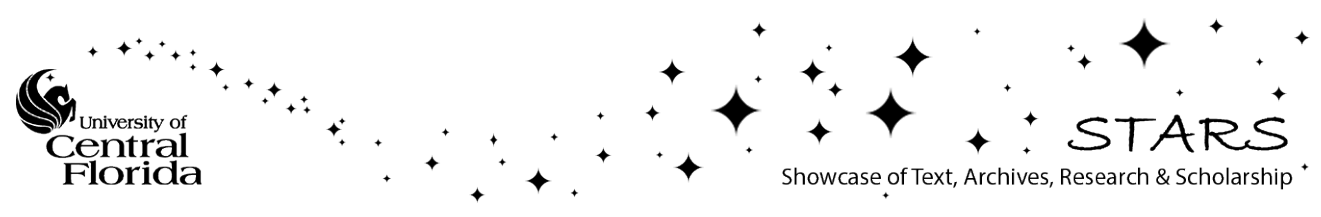




\title{
COMPUTATIONAL FLUID DYNAMICS UNCERTAINTY ANALYSIS FOR PAYLOAD FAIRING SPACECRAFT ENVIRONMENTAL CONTROL SYSTEMS
}

by

\section{CURTIS EDWARD GROVES}

B.S. West Virginia University, 2008

B.S. West Virginia University, 2008

M.S. University of Central Florida, 2012

\begin{abstract}
A dissertation submitted in partial fulfillment of the requirements for the degree of Doctor of Philosophy

in the Department of Mechanical and Aerospace Engineering in the College of Engineering and Computer Science at the University of Central Florida Orlando, Florida
\end{abstract}

Spring Term

2014

Major Professor: Alain Kassab 
(C) 2014 Curtis Edward Groves 


\begin{abstract}
Spacecraft thermal protection systems are at risk of being damaged due to airflow produced from Environmental Control Systems. There are inherent uncertainties and errors associated with using Computational Fluid Dynamics to predict the airflow field around a spacecraft from the Environmental Control System. This paper describes an approach to quantify the uncertainty in using Computational Fluid Dynamics to predict airflow speeds around an encapsulated spacecraft without the use of test data. Quantifying the uncertainty in analytical predictions is imperative to the success of any simulation-based product. The method could provide an alternative to traditional "validation by test only" mentality. This method could be extended to other disciplines and has potential to provide uncertainty for any numerical simulation, thus lowering the cost of performing these verifications while increasing the confidence in those predictions.
\end{abstract}

Spacecraft requirements can include a maximum airflow speed to protect delicate instruments during ground processing. Computational Fluid Dynamics can be used to verify these requirements; however, the model must be validated by test data. This research includes the following three objectives and methods. Objective one is develop, model, and perform a Computational Fluid Dynamics analysis of three (3) generic, non-proprietary, environmental control systems and spacecraft configurations. Several commercially available and open source solvers have the capability to model the turbulent, highly three-dimensional, incompressible flow regime. The proposed method uses FLUENT, STARCCM+, and OPENFOAM. Objective two is to perform an uncertainty analysis of the Computational Fluid Dynamics model using the 
methodology found in "Comprehensive Approach to Verification and Validation of Computational Fluid Dynamics Simulations". This method requires three separate grids and solutions, which quantify the error bars around Computational Fluid Dynamics predictions. The method accounts for all uncertainty terms from both numerical and input variables. Objective three is to compile a table of uncertainty parameters that could be used to estimate the error in a Computational Fluid Dynamics model of the Environmental Control System /spacecraft system.

Previous studies have looked at the uncertainty in a Computational Fluid Dynamics model for a single output variable at a single point, for example the re-attachment length of a backward facing step. For the flow regime being analyzed (turbulent, three-dimensional, incompressible), the error at a single point can propagate into the solution both via flow physics and numerical methods. Calculating the uncertainty in using Computational Fluid Dynamics to accurately predict airflow speeds around encapsulated spacecraft in is imperative to the success of future missions. 
This work is dedicated to my wife: Emily...

Thank you for always being there and not settling... 


\section{ACKNOWLEDGMENTS}

I would like to thank my advisor and mentor, Dr. Alain Kassab, for teaching and providing guidance. He allowed me to pursue research and continue working at the Kennedy Space Center. His patience and helpfulness is unmatched, and I am grateful to have met and worked with him. The University of Central Florida (UCF) has found a wonderful asset with Dr. Kassab and his compassion to student performance.

I would like to thank the PhD committee members Dr. Tuhin Das, Dr. Jeffery Kauffman, and and Dr. Brian Moore for their feedback and helpfulness while completing the dissertation. Every committee member made significant contributions to the research and I am thankful for their experience and willingness to help.

My sincere thanks to my Supervisor, Dr. Paul Schallhorn (Environments and Launch Approval Branch Chief), and my Leads, Lennie Duncil (Fluids Lead) and Larry Craig (Launch Approval Lead), at the Kennedy Space Center for the opportunity to work on the PhD. Without Paul's guidance, I never would have continued my education or pursued a $\mathrm{PhD}$. Without the Leads willingness to allow me to spend a year away from work through Kennedy Graduate Fellowship Progam (KGFP), I would never have been able to complete the requirements of the degree.

My sincere appreciations to NASA's Launch Service Program (LSP) and Kennedy Space Center (KSC) for funding the research, my time, and providing the computational resources needed to complete the simulations. The KGFP Program and management's support for employees to pursue advanced degrees while working is unsurpassed and I am sincerely thankful to work for 
National Aeronautics and Space Administration (NASA), Kennedy Space Center (KSC), and the Launch Services Program (LSP) and grateful to have had this opportunity. 


\section{TABLE OF CONTENTS}

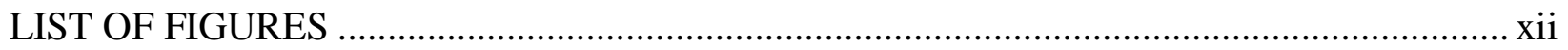

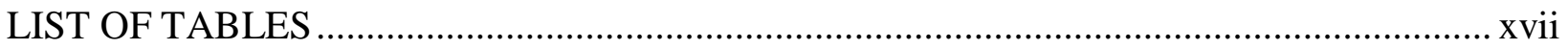

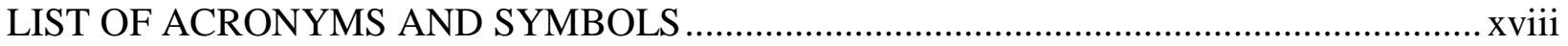

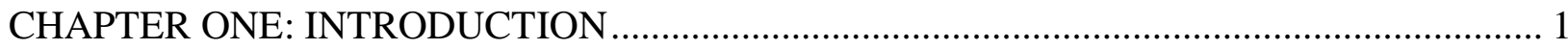

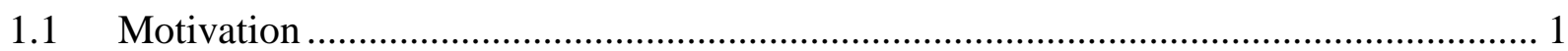

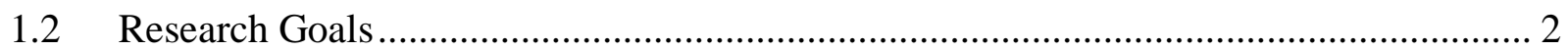

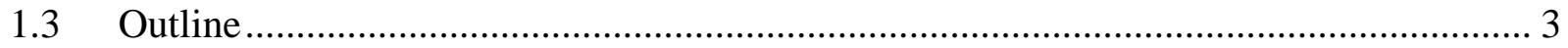

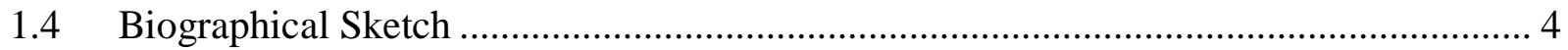

CHAPTER TWO: LITERATURE REVIEW ..................................................................... 5

2.1 Summary of Literature Review ......................................................................... 9

2.2 Summary of the State of the Art CFD Uncertainty Analysis .................................. 9

2.3 Proposed Methodology without Test Data........................................................ 12

CHAPTER THREE: APPLYING THE “STATE OF THE ART” CFD UNCERTAINTY

ANALYSIS TO A BACKWARD FACING STEP ......................................................... 16

3.1 Grid Refinement Study and Velocity Prediction.................................................. 16

3.2 CFD Uncertainty Analysis of Backward Facing Step............................................ 17

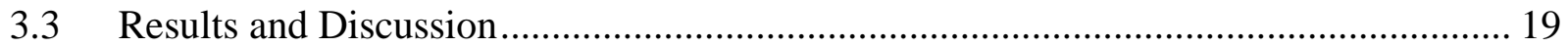




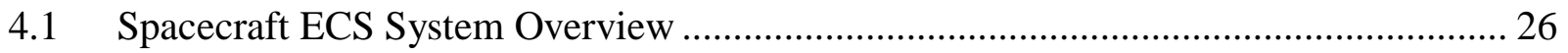

4.1.1 Publically Available Information on EELV ECS Systems .............................. 28

4.1.2 Modeling and CFD Analysis of (3) Generic Non-Proprietary Environmental

Control System and Spacecraft Configurations ....................................................... 30

CHAPTER FIVE: COMPUTATIONAL FLUID DYNAMICS UNCERTAINTY ANALYSIS. 38

5.1 Interpolation Scheme Needed for CFD Uncertainty Analysis ................................... 39

5.2 Feasibility of Using Richardson's Extrapolation for Entire Computational Domain .... 54

5.3 Proposed CFD Uncertainty Method Compared to Exact Solution -Laminar Flow

Between Parallel, Stationary Plates ........................................................................... 58

5.4 Proposed CFD Uncertainty Method Applied to Heat Transfer over a Flat Plate.......... 69

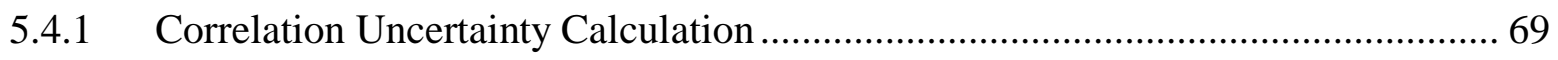

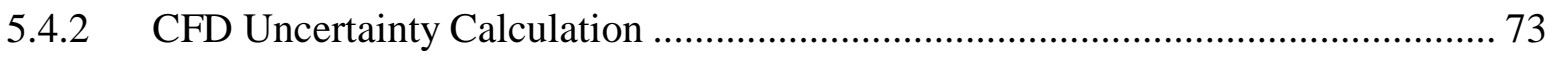

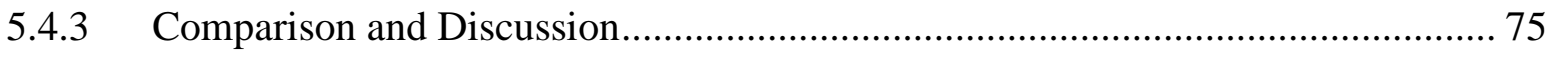

CHAPTER SIX: DEMONSTRATION AND IMPLEMENTATION OF THE PROPOSED CFD

UNCERTAINITY METHOD FOR SPACECRAFT ECS SYSTEMS ................................. 79

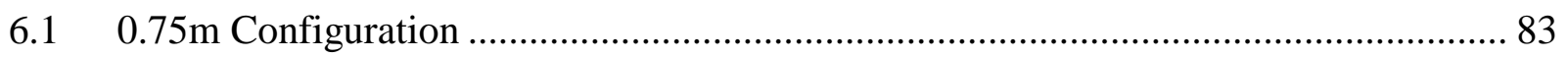

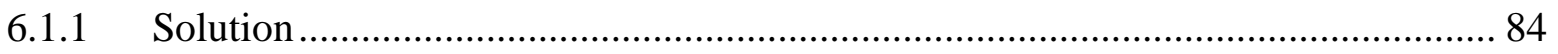

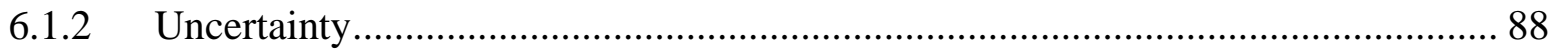




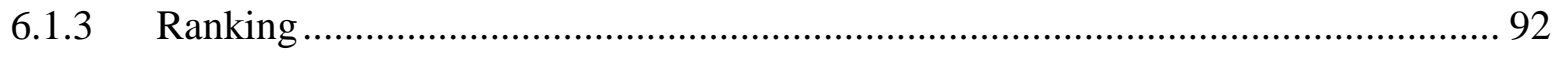

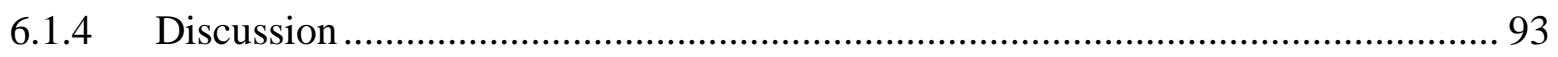

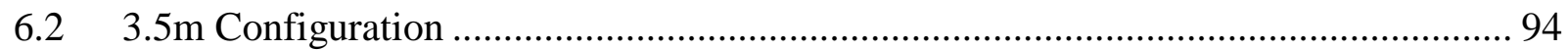

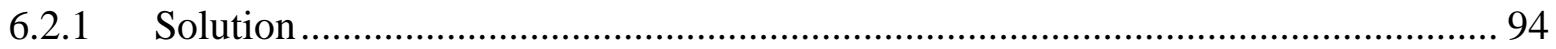

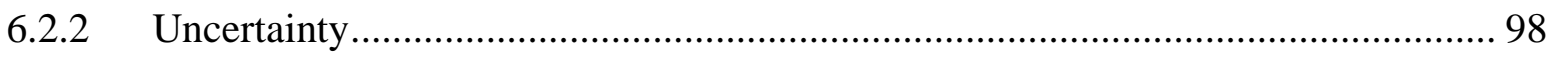

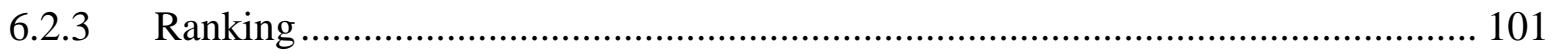

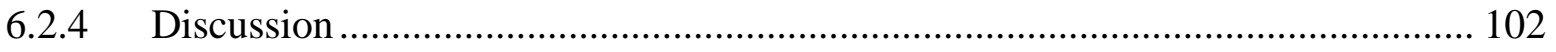

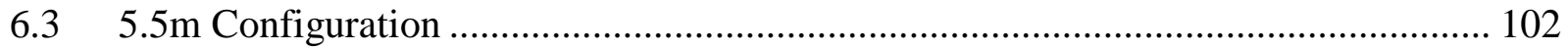

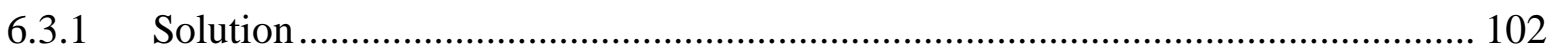

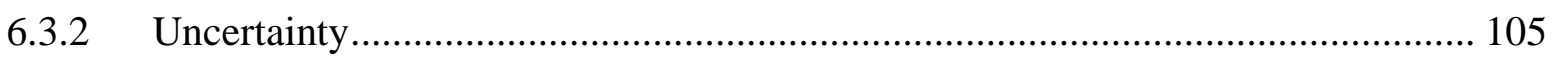

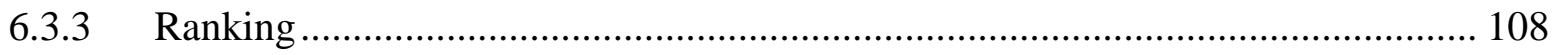

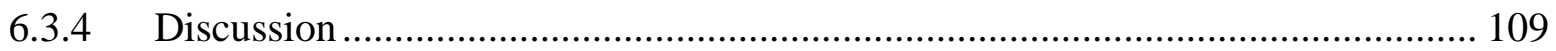

6.4 ECS System Experimental Comparison ........................................................................ 110

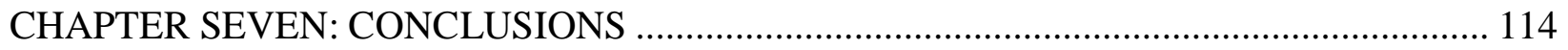

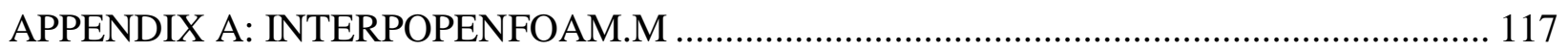

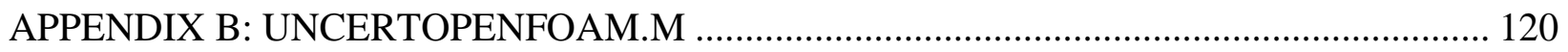

Appendix B.1 Uncertopenfoam.M -Max,Min,Uncert ....................................................... 122

Appendix B.2 Uncertopenfoam.M - Write Min …………………................................... 124

Appendix B.3 Unceropenfoam.M - Write Max.................................................................. 126 
Appendix B.4 Uncertopenfoam.M -Write Middle

Appendix B.5 Uncertopenfoam.M - Write Uncertainty ....

Appendix B.6 Uncertopenfoam.M - Write Uncertainty Low.

Appendix B.7 Uncertopenfoam.M - Write Uncertainty High

APPENDIX C: RKOPENFOAM.M - CALCULATE RK AND WRITE OPENFOAM .......... 136 APPENDIX D: NONDIMRANK.M - NON-DIMENSIONALIZATION AND RANKING OF INPUT UNCERTAINTY 138

APPENDIX E: FLAT PLATE UNCERTAINTY MATLAB (FLATPLAT.M) ..................... 146

Appendix E.1 Heat Transfer Correlation Uncertainty Calculation (Uncert_Hi.M) ........... 150

Appendix E.2 Remove Duplicates Script (Rem_Dup_2.M) ........................................ 153

APPENDIX F: SUMMARY OF AUTHORS PRIOR WORK AND BACKGROUND. 155

LIST OF REFERENCES 158 


\section{LIST OF FIGURES}

Figure 1: Convergence conditions for the backward facing step - Grid refinement .................. 16

Figure 2: Velocity Magnitude for Flow over a Backward Facing Step - 1,192,000 cells........... 17

Figure 3: Epsilon Turbulent Mixing Length Dissipation Rate Inlet - Velocity Uncertainty

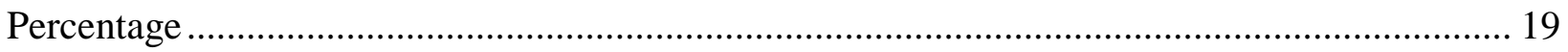

Figure 4: kTurbulent Intensity Kinetic Energy Inlet - Velocity Uncertainty Percentage ........... 20

Figure 5: Pressure Outlet - Velocity Uncertainty Percentage ............................................ 20

Figure 6: Velocity Inlet - Velocity Uncertainty Percentage................................................ 21

Figure 7: Kinematic Viscosity - Velocity Uncertainty Percentage ...................................... 21

Figure 8: Grid Size - Velocity Uncertainty Percentage ................................................... 22

Figure 9: Turbulence Models - Velocity Uncertainty Percentage .......................................... 22

Figure 10: Solver - Velocity Uncertainty Percentage ................................................... 23

Figure 11: Numerical - Velocity Uncertainty Percentage .................................................. 23

Figure 12: Velocity Prediction and Uncertainty Plot for ke-realizable Turbulence Model......... 24

Figure 13: Velocity Uncertainty Plot for ke-realiable Turbulence Model................................ 24

Figure 14: Environmental Control System (ECS) Overview [22] ....................................... 26

Figure 15: Environmental Control System (ECS) Airflow Swirl [22] ................................... 26

Figure 16: Environmental Control System (ECS) Airflow Testing [24] ................................ 27

Figure 17: 0.75m Diameter Fairing CAD Model of ECS / Spacecraft .................................. 31

Figure 18: 3.5m Diameter Fairing CAD Model of ECS / Spacecraft ....................................... 31

Figure 19: 5.5m Diameter Fairing CAD Model of ECS / Spacecraft ..................................... 32 
Figure 20: OpenFOAM SnappyHex Mesh Modeling CAD Model of ECS / Spacecraft - 0.75m

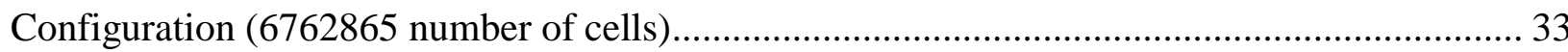

Figure 21: OpenFOAM SnappyHex Mesh Modeling CAD Model of ECS / Spacecraft - 3.5m

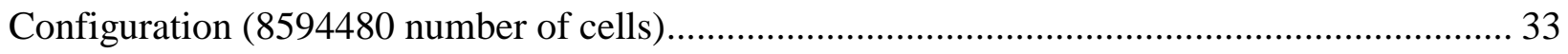

Figure 22: OpenFOAM SnappyHex Mesh Modeling CAD Model of ECS / Spacecraft - 5.5m

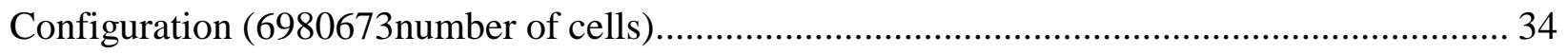

Figure 23: OpenFOAM SimpleFOAM Velocity Contours of ECS / Spacecraft - 0.75m

Configuration (6762865 number of cells) ....................................................................... 35

Figure 24: OpenFOAM SimpleFOAM Velocity Contours of ECS / Spacecraft - 3.5m

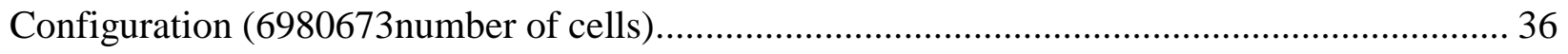

Figure 25: OpenFOAM SimpleFOAM Velocity Contours of ECS / Spacecraft $-5.5 \mathrm{~m}$

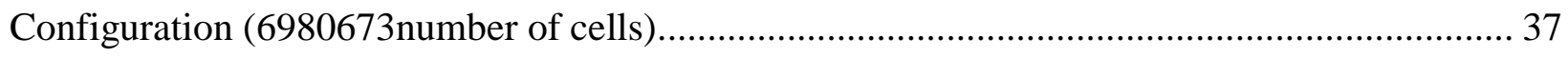

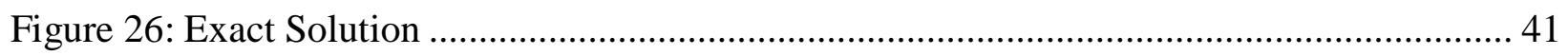

Figure 27: CFD Results (coarse, medium, fine) vs. Exact Solution ........................................ 42

Figure 28: "nearest" interpolation of CFD Results (coarse, medium, fine) vs. Exact Solution ... 43

Figure 29: "linear" interpolation of CFD Results (coarse, medium, fine) vs. Exact Solution...... 44

Figure 30: "cubic" interpolation of CFD Results (coarse, medium, fine) vs. Exact Solution ...... 45

Figure 31: 3D Linear Interpolation Contour Plot vs. Fine Solution ........................................ 47

Figure 32: 3D Linear Interpolation Line Plot ............................................................... 47

Figure 33: 3D Linear Interpolation Line Plot vs. Fine, Medium, and Coarse Solutions ............. 48

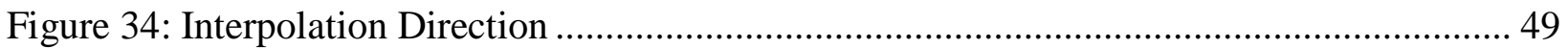

Figure 35: Interpolation Direction onto Fine Grid ........................................................ 50 


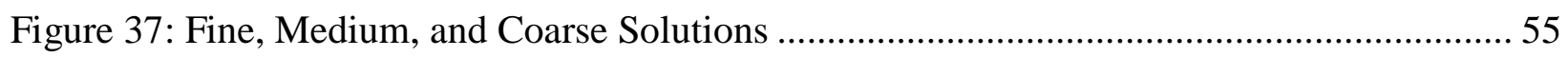

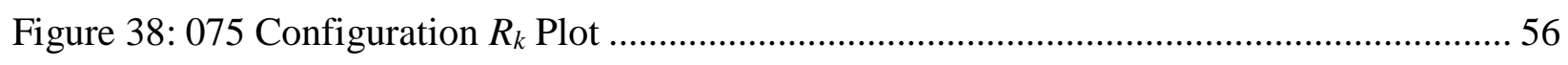

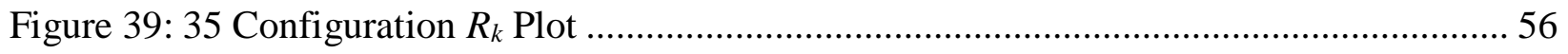

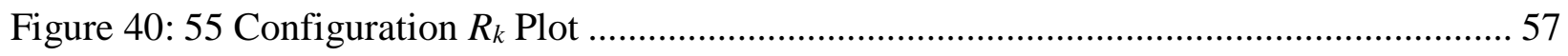

Figure 41: Exact Solution vs. CFD with Uncertainty (Centerline Velocity) - Grid ................... 59

Figure 42: Exact Solution vs. CFD with Uncertainty (Centerline Velocity) - Grid and Inlet

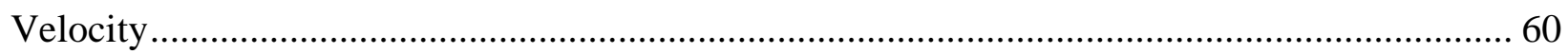

Figure 43: Exact Solution vs. CFD with Uncertainty (Centerline Velocity) - Grid, Inlet Velocity,

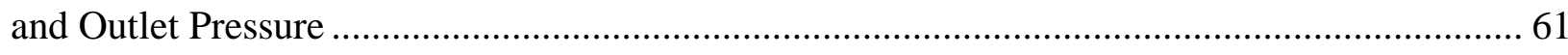

Figure 44: Exact Solution vs. CFD with Uncertainty (Centerline Velocity) - Grid, Inlet Velocity,

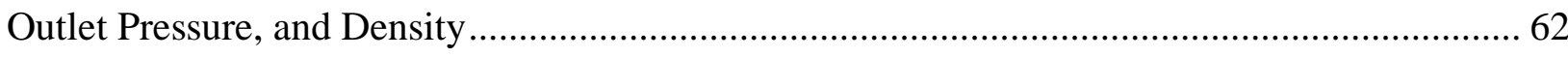

Figure 45: Exact Solution vs. CFD with Uncertainty (Centerline Velocity) - Grid, Inlet Velocity,

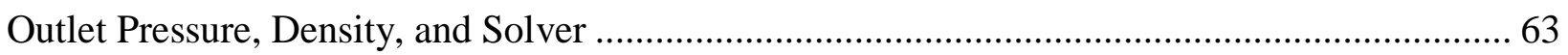

Figure 46: Exact Solution vs. CFD with Uncertainty (Parallel Plates - Half of Domain) - Grid,

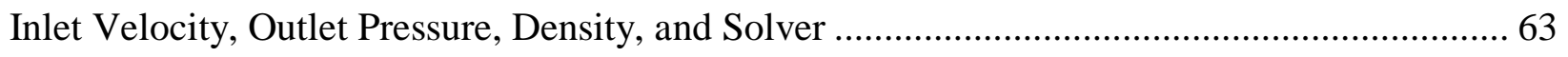

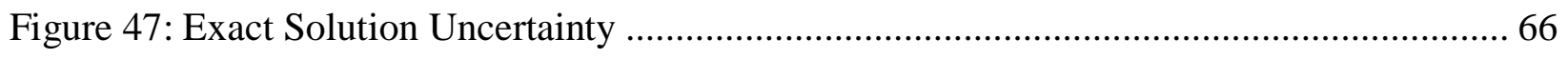

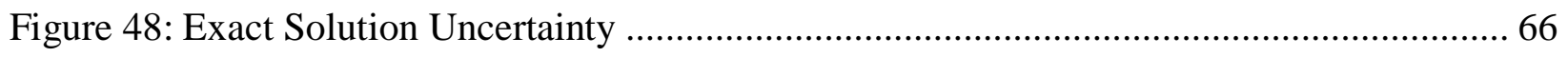

Figure 49: Exact Solution Uncertainty vs. Numerically Derived Uncertainty ......................... 67

Figure 50: Exact Solution with Uncertainty vs. Numerically Derived Uncertainty ................... 67

Figure 51: Exact Solution Uncertainty vs. Numerically Derived Uncertainty (with k=1.43) ...... 68

Figure 52: Exact Solution Uncertainty vs. Numerically Derived Uncertainty (with k=1.43) ..... 68 
Figure 53: Heat Transfer Coefficient - Correlation ................................................................. 72

Figure 54: Heat Transfer Coefficient and Uncertainty - Correlation............................................ 73

Figure 55: Heat Transfer Coefficient and Uncertainty - CFD Data ............................................. 74

Figure 56: Heat Transfer Coefficient and Uncertainty - CFD Data ............................................ 75

Figure 57: Heat Transfer Coefficient Uncertainty - CFD Data vs. Correlation .............................. 76

Figure 58: Heat Transfer Coefficient with Uncertainty - CFD Data vs. Correlation..................... 77

Figure 59: Heat Transfer Coefficient Uncertainty - CFD Data $(\mathrm{k}=1.2)$ vs. Correlation ............... 78

Figure 60: CFD Results for 0.75m Configuration - Contour Plot............................................ 84

Figure 61: CFD Results for 0.75m Configuration - Line Plot (m/s) ......................................... 85

Figure 62: CFD Results for 0.75m Configuration - Line Plot (m/s) ........................................... 86

Figure 63: CFD Results for 0.75m Configuration - Line Plot (m/s) ......................................... 87

Figure 64: CFD Results for 0.75m Configuration - Line Plot (m/s) ......................................... 88

Figure 65: CFD Results and Uncertainty for 0.75m Configuration - Contour Plot (m/s)............ 89

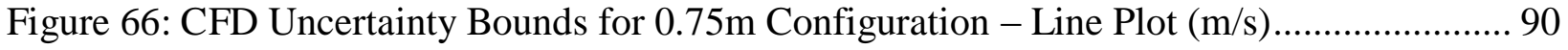

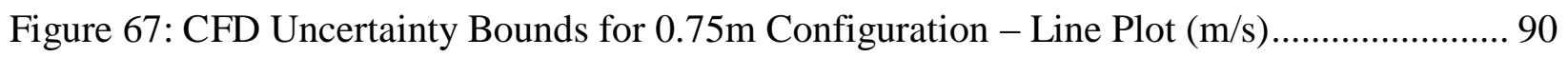

Figure 68: CFD Uncertainty Bounds for 0.75m Configuration - Line Plot $(\mathrm{m} / \mathrm{s}) \ldots \ldots \ldots \ldots \ldots \ldots \ldots \ldots . . . .191$

Figure 69: CFD Uncertainty Bounds for 0.75m Configuration - Line Plot $(\mathrm{m} / \mathrm{s}) \ldots \ldots \ldots \ldots \ldots \ldots \ldots \ldots . . . .91$

Figure 70: CFD Results for 3.5m Configuration - Contour Plot (m/s) ...................................... 95

Figure 71: CFD Results for 3.5m Configuration - Line Plot (m/s) .......................................... 96

Figure 72: CFD Results for 3.5m Configuration - Line Plot (m/s) ............................................ 97

Figure 73: CFD Results and Uncertainty for 3.5m Configuration - Contour Plot (m/s)............. 98

Figure 74: CFD Uncertainty Bounds for 3.5m Configuration - Line Plot (m/s)........................ 99 
Figure 75: CFD Uncertainty Bounds for 3.5m Configuration - Line Plot (m/s)................... 100

Figure 76: CFD Results for 5.5m Configuration - Contour Plot $(\mathrm{m} / \mathrm{s})$................................. 103

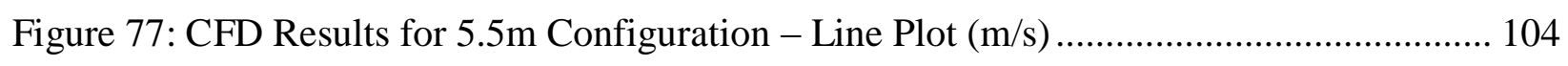

Figure 78: CFD Results for 5.5m Configuration - Line Plot $(\mathrm{m} / \mathrm{s})$..................................... 104

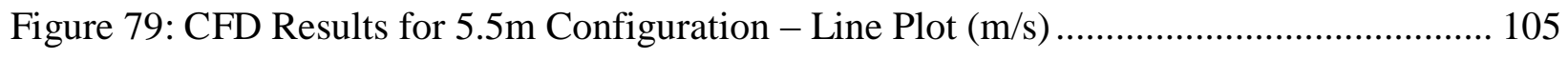

Figure 80: CFD Results for 5.5m Configuration - Line Plot (m/s) .................................. 105

Figure 81: CFD Results and Uncertainty for 5.5m Configuration - Contour Plot (m/s).......... 106

Figure 82: CFD Results and Uncertainty for 5.5m Configuration - Line Plot (m/s) .............. 107

Figure 83: CFD Results and Uncertainty for 5.5m Configuration - Line Plot (m/s) ............. 107

Figure 84: CFD Results and Uncertainty for 5.5m Configuration - Line Plot (m/s) .............. 108

Figure 85: CFD Results and Uncertainty for 5.5m Configuration - Line Plot (m/s) .............. 108

Figure 86: Proposed CFD Uncertainty Method vs. Experimental Data constant x .................. 111

Figure 87: Proposed CFD Uncertainty Method vs. Experimental Data constant z ................. 112

Figure 88: CFD Model and Experimental Apparatus [24] ................................................ 113 


\section{LIST OF TABLES}

Table 1: Student T Distribution, k Values [20].................................................................. 15

Table 2: Uncertainty Variables, Xi - Backward Facing Step ............................................ 18

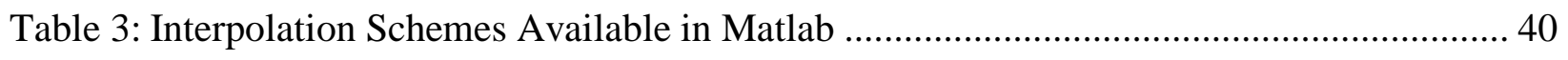

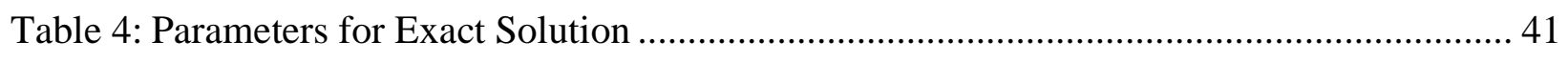

Table 5: Results Interpolating to Fine Grid for Flow Between Parallel Plates......................... 51

Table 6: Results Interpolating to Coarse Grid for Flow Between Parallel Plates ....................... 52

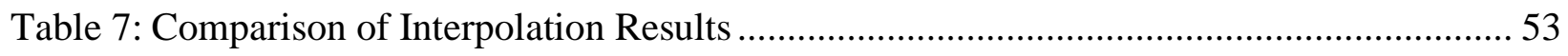

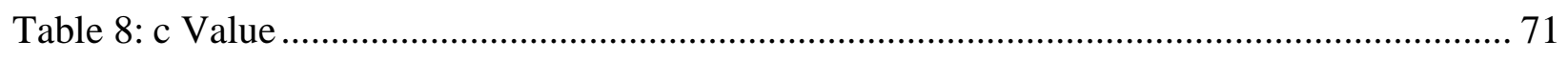

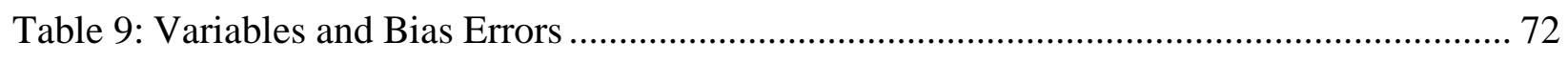

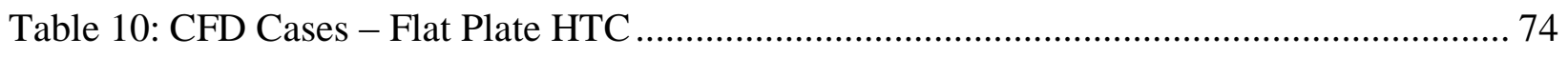

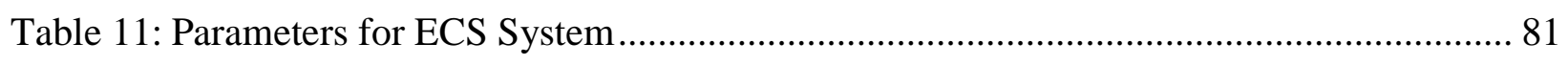

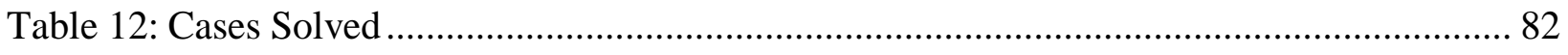

Table 13: 0.75m Configuration - Non-Dimensional Ranking of Uncertainty ......................... 93

Table 14: 3.5m Configuration - Non-Dimensional Ranking of Uncertainty .......................... 101

Table 15: 5.5m Configuration - Non-Dimensional Ranking of Uncertainty ......................... 109 


\section{LIST OF ACRONYMS AND SYMBOLS}

\begin{tabular}{|c|c|c|}
\hline $\mathbf{a}$ & $=$ & channel width \\
\hline c & $=$ & heat transfer coefficient correlation factor \\
\hline$\delta_{D}$ & $=$ & experimental error \\
\hline$\delta_{\text {input }}$ & $=$ & input error \\
\hline$\delta_{\text {model }}$ & $=$ & modeling error \\
\hline$\delta_{\text {num }}$ & $=$ & numerical error \\
\hline$\delta_{s}$ & $=$ & simulated error \\
\hline $\mathbf{D}$ & $=$ & experimental value \\
\hline dp/dx & $=$ & pressure gradient \\
\hline$\varepsilon_{21}$ & $=$ & solution changes medium to fine grid \\
\hline$\varepsilon_{32}$ & $=$ & solution changes coarse to medium grid \\
\hline$e_{a}^{21}$ & $=$ & extrapolated error \\
\hline $\mathbf{E}$ & $=$ & comparison error \\
\hline EELV & $=$ & evolved expendable launch vehicle \\
\hline$G C I_{\text {fine }} e^{21}$ & $=$ & grid convergence index \\
\hline h & $=$ & representative grid size \\
\hline h & $=$ & heat transfer coefficient \\
\hline 1 & $=$ & characteristic length \\
\hline $\mathbf{p}$ & $=$ & observed order \\
\hline $\boldsymbol{\rho}$ & $=$ & density \\
\hline
\end{tabular}




\begin{tabular}{|c|c|c|}
\hline $\mathbf{R}_{\mathbf{k}}$ & $=$ & convergence parameter \\
\hline$r_{21}$ & $=$ & ratio of grid sizes between grid 1 and 2 \\
\hline $\mathbf{r}_{32}$ & $=$ & ratio of grid sizes between grid 3 and 2 \\
\hline $\mathbf{S}$ & $=$ & simulated result \\
\hline $\mathbf{S}_{\mathbf{k} 1}$ & $=$ & solution variable for fine grid \\
\hline$S_{k 2}$ & $=$ & solution variable for medium grid \\
\hline$S_{k 3}$ & $=$ & solution variable for coarse grid \\
\hline$S_{e x t}^{21}$ & $=$ & extrapolated solution variable \\
\hline$S_{L}$ & $=$ & lowest solution variable \\
\hline Su & $=$ & highest solution variable \\
\hline Uoscillatory & $=$ & uncertainty for oscillatory portion of the solution \\
\hline $\mathbf{U}_{\text {monotonic }}$ & $=$ & uncertainty for monotonic portion of the solution \\
\hline Uh & $=$ & uncertainty in heat transfer coefficient \\
\hline Uu & $=$ & uncertainty in velocity profile between parallel plates \\
\hline $\mathbf{u}$ & $=$ & velocity profile between parallel plates \\
\hline Uinput & $=$ & input uncertainty \\
\hline uD & $=$ & experimental uncertainty \\
\hline $\mathbf{U}_{\text {num }}$ & $=$ & numerical uncertainty \\
\hline $\mathbf{U}_{\text {val }}$ & $=$ & validation uncertainty \\
\hline $\boldsymbol{\mu}$ & $=$ & viscosity \\
\hline $\mathbf{V}$ & $=$ & velocity \\
\hline$\overline{\boldsymbol{V}}$ & $=$ & average velocity \\
\hline
\end{tabular}


distance from wall 


\section{CHAPTER ONE: INTRODUCTION}

\subsection{Motivation}

Delicate spacecraft instruments are needed for satellite technology enhancement of agricultural yield, environment sustainability, or telecommunications. Before spacecraft are released into orbit to complete their science goals, the spacecraft must survive the ground and launch environments. Environmental Control Systems (ECS) systems supply air to keep the spacecraft cool, dry, and clean. Delicate spacecraft instruments are sensitive to high velocity flow from the ECS systems and manufactures set impingement requirements to protect these instruments. CFD is often chosen to complete verifications of the impingement requirements rather than testing. Using CFD to predict the airflow field around a spacecraft enclosed in a fairing has been documented and validated using test data [1], [2].

The problem is there are inherent uncertainties and errors associated with using CFD to predict the airflow field, and there is no standard method for evaluating uncertainty in the CFD community [3]. Some potentials errors include physical approximation error, computer round-off error, iterative convergence error, discretization errors, computer programming errors, and usage errors [4]. An uncertainty, as defined by the American Institute of Aeronautics and Astronautics (AIAA), is a potential deficiency in any phase or activity of modeling and simulation that is due to the lack of knowledge [5]. An example of an uncertainty in performing a CFD analysis is turbulence modeling [4]. There is a lot about turbulence modeling that is not understood [4]. There has been progress in estimating the uncertainty of CFD, but the approaches have not converged [3]. 
CFD is used primarily for analytical predictions of the velocity, heat transfer coefficient, and pressure. CFD is the current state of the art and industry standard used for spacecraft ECS flow analysis; however CFD has many challenges. The users must select the appropriate models to characterize their specific problem. The proposed research will use different turbulence models as an input uncertainty to help the community evaluate the accuracy of turbulence modeling. There are many other input variables. These include boundary conditions, wall functions, fluid properties, turbulence models, solution schemes, solvers, mesh, and numerical calculations. The current state of the art uncertainty analysis will evaluate each of the error sources and provide the corresponding uncertainty of the velocity around a spacecraft due to the ECS system. No one to date has ever calculated the uncertainty in using CFD to predict the velocity of spacecraft/ECS systems for the entire domain. The benefit to the community is a proven, documented approach, and provided table of all uncertainty variables, which can be used to estimate the error in a velocity prediction.

\subsection{Research Goals}

The work focuses on solving the following issues:

1. Demonstrate a CFD Uncertainty Analysis for 3-D, low speed, incompressible, highly turbulent, internal flow can be calculated for an entire simulation domain.

2. Investigate a higher order interpolation scheme to be used for grid interpolations and uncertainty quantification. 
3. Investigate the applicability of using the ASME 5-Step procedure for the entire computational domain to estimate numerical uncertainties.

4. Calculate the uncertainty in using different turbulent models.

5. Demonstrate this method can contribute to the study of importance of input parameters in CFD.

6. Compile a table for uncertainty estimates by input parameter. The table will benefit the community by providing an uncertainty estimate in lieu of running hundreds of CFD simulations.

7. Demonstrate the ability to use OPENFOAM to calculate the velocity field of an Environmental Control System.

8. Compare the results of OPENFOAM verses an industry standard CFD software program (ie FLUENT and STARCCM+).

\subsection{Outline}

Chapter Two is a thorough Literature Review to find the "current state of the art" method for performing Computational Fluid Dynamics (CFD) Uncertainty Analysis. Application of the current state of the art method to a simple backward facing academic problem is discussed in Chapter Three. Modeling of the Spacecraft/ECS System is provided in Chapter Four. Chapter Five describes the proposed CFD Uncertainty Approach. Chapter Five applies the proposed CFD Uncertainty Approach to the simple problems of fully developed flow between parallel plates and heat transfer over a flat plate. The results are compared to an exact solution and experimental data. 
Chapter 6 documents the approach for spacecraft/ECS systems. The conclusions are provided in Chapter Seven.

\subsection{Biographical Sketch}

The author, Mr. Curtis Groves, is a PhD candidate at the University of Central Florida. Mr. Groves has worked for NASA at the Kennedy Space Center in the Launch Services Program since 2006 where he performs independent verifications of NASA's science payload requirements. Mr. Groves has performed ECS impingement verifications for the following missions: GLORY, MSL, TDRSS-K/L, and IRIS and external aerodynamics verification on the Atlas V vehicle. Mr. Groves completed dual Bachelor's Degrees in aerospace engineering and mechanical engineering from West Virginia University and graduated Summa Cum Laude. Mr. Groves has graduated from the University of Central Florida with a master's in aerospace engineering in May 2012 and is working to complete a $\mathrm{PhD}$ in May 2014. A summary of Mr. Groves' background is provided in Appendix F. Mr. Groves has research interests in Computational Fluid Dynamics, Turbulence Modeling, Uncertainty Analysis, External Aerodynamics, Spacecraft Venting, Environmental Control Systems, and Heat Transfer. 


\section{CHAPTER TWO: LITERATURE REVIEW}

A literature review was performed to determine the "State of the Art" method for calculating CFD uncertainties. CFD is extensively used in industry, government, and academia to design, investigate, operate, and improve understanding of fluid physics [5]. The rate of growth in using CFD as a research and engineering tool will be directly proportional to the level of credibility the simulation can produce [5]. One needs to evaluate the uncertainty in the results of a CFD simulation to postulate a level of credibility. In 1986, The American Society of Mechanical Engineers (ASME) Journal of Fluids Engineering published a policy statement stating the need for quantification of numerical accuracy [3]. These statements lead to research on the best method to determine numerical uncertainty. In 1995, Celik and Zhang published "Calculation of Numerical Uncertainty Using Richardson Extrapolation: Application to Some Turbulent Flow Calculations" which used Richardson's Extrapolation method to estimate the uncertainty in CFD [6]. In 1997, Roache published "Quantification of Uncertainty in Computational Fluid Dynamics" [7]. Roaches research also used the Richardson Extrapolation method to quantify CFD uncertainties.

In 1998, the AIAA has published a "Guide for the Verification and Validation of Computational Fluid Dynamics Simulations" [5]. This document provides guidelines for assessing credibility via verification and validation [5]. The document does not recommend standards due to issues not yet resolved, but defines several terms [5]. "Uncertainty is defined as a potential deficiency in any phase or activity of the modeling process that is due to lack of knowledge [5]." "Error is defined as a recognizable deficiency in any phase or activity of modeling and simulation 
that is not due to lack of knowledge [5]." "Prediction is defined as the use of a CFD model to foretell the state of a physical system under conditions for which the CFD model has not been validated [5]." Uncertainty and error are normally linked to accuracy in modeling and simulation [5]. The guide defines four predominate error sources: insufficient spatial discretization convergence, insufficient temporal discretization convergence, lack of iterative convergence, and computer programming, but does not make claims about the accuracy of predictions [5]. The guide emphasizes that systematically refining the grid size and time step is the most important activity in verification [5]. Once the grid has been refined to where the discretization error is in the asymptotic region, Richardson's extrapolation can be used to estimate zero-grid spacing [5]. A sensitivity analysis and uncertainty analysis are two methods for determining the uncertainty in CFD [5]. The validation test compares a CFD solution to experimental data [5]. The guide has outlined the terms and an overall structure to performing validation, but does not offer a quantitative method.

In 1999, Stern, Wilson, Coleman, and Paterson, E. G., published Iowa Institute of Hydraulic Research (IIHR) Report No. 407 titled "Verification and Validation of CFD Simulations" [8]. In 2001, the American Society of Mechanical Engineers (ASME) Journal of Fluids Engineering published a "Comprehensive Approach to Verification and Validation of CFD Simulations" in an attempt to provide a comprehensive framework for overall procedures and methodology [9]. Two papers were published on the subject in Parts I [9] and Parts II [10] and used the methodology documented in IIHR Report 407. Numerical errors and uncertainties in CFD can be estimated using iterative and parameter convergence studies [9]. The method uses three convergence conditions as possible in estimating uncertainties; (1) monotonic convergence which uses 
Richardson's extrapolation, (2) oscillatory convergence which uses the upper and lower bounds to estimate uncertainty, (3) divergence in which errors and uncertainties cannot be estimated [9]. The literature provides an approach for estimating errors and uncertainties in CFD simulations for each of the three cases [9], [10], [8]. The approach uses Richardson's extrapolation, which is not new, however; the method has been extended to use input parameters and correction factors to estimate errors and uncertainties [9], [10], [8]. The method examines two sources for error and uncertainty: modeling and simulation. Examples of modeling errors include geometry, mathematical equations, boundary conditions, turbulence models, etc. [9]. Examples of numerical errors include discretization, artificial dissipations, incomplete iterative and grid convergence, lack of conservation of mass, momentum, energy, internal and external boundary non-continuity, computer round-off etc. [4]. The method lacks correlations among errors and assumes these are negligible, which may be inappropriate for some circumstances [9]. Additionally, the method provides a quantitative approach for determining the iterative convergence uncertainty [9]. Iterative Convergence must be evaluated and is typically done by monitoring the residuals order of magnitude drop graphically [9]. For oscillatory convergence, the deviation of a residual from the mean provides estimates of the iterative convergence [9]. This is based on the range of the maximum $S_{U}$ and minimum $S_{L}$ values [9]. For convergent iterative convergence, a curve-fit is used [9]. For a mixed convergent/oscillatory, iterative convergence is estimated using the amplitude and the maximum and minimum values [9]. A method for confirming validation is presented as compared to experimental data [9].

In 2008, the International Towing Tank Conference (ITTC) has published "Recommended Procedures and Guidelines - Uncertainty Analysis in CFD Verification and Validation 
Methodology and Procedures" [11]. The ITTC guide was largely based off of the methodology and procedures presented in the ASME Journal of Fluids Engineering a "Comprehensive Approach to Verification and Validation of CFD Simulations" [11]. Also in 2008, the ASME Journal of Fluids Engineering published a "Procedure for Estimating and Reporting of Uncertainty Due to Discretization in CFD Applications" [12].

In 2011, the National Energy Technology Laboratory (NETL) conference proceedings held a major section related to CFD Uncertainty Calculation [13]. Celik presented "Critical Issues with Quantification of Discretization Uncertainty in CFD" [13]. The proceedings were based off of the ASME “Comprehensive Approach to Verification and Validation of CFD Simulations” [9].

In 2009, the American Society of Mechanical Engineers published "Standard for Verification and Validation in Computational Fluid Dynamics and Heat Transfer", ASME V\&V 20-2009 [14]. The standard provides a procedure for estimating the uncertainty and is based off of the literature presented above.

In 2010, Roy published "Review of Discretization Error Estimators in Scientific Computing" [15]. The paper is quite extensive and discusses the methodology of using the Grid Convergence Index (GCI) which is the basis of the ASME standard and previous research. Roy references the original paper by Roache in 1994 [16]. Roache's GCI method is based off of the original Richardson's Extrapolation in 1911 and 1927 which is a basic concept of having separate solutions for systematically refined grids, one can approximate the exact solution from two systematically refined meshes [15], [17], [18]. The assumptions to Richardson's extrapolation are as follows: 1) both discrete solutions are in the asymptotic range, 2) the meshes have a uniform spacing, 3) coarse 
and fine meshes are related through systematic refinement, 4) solutions are smooth, 5) other sources of numerical error are small [15]. Roy further provides a section on open research issues [15]. These include singularities and discontinuities, oscillatory convergence with mesh refinement, multi-scale models, and coarse grid error estimators [15].

\subsection{Summary of Literature Review}

A thorough literature review has been performed to determine the best method to evaluate the uncertainty in CFD predictions. Both major journals in mechanical and aerospace engineering, AIAA and ASME, have published articles on this subject. The ASME Standard methodology has been adopted by many researchers and provides a detailed approach to calculate uncertainty in CFD from different levels of grid refinement and input parameter studies. The method published by the ASME Journal of Fluids Engineering (ASME V\&V 20-2009 [14]) is the state of the art for determining the uncertainty in CFD predictions and was used for the completed research problem.

\subsection{Summary of the State of the Art CFD Uncertainty Analysis}

A summary of the ASME V\&V 20-2009 "Standard for Verification and Validation in Computational Fluid Dynamics and Heat Transfer" is provided in this section. The methodology is as follows. The validation comparison error, E, is the difference between the

simulated result, S, and the experimental value, D [14]. The goal is to characterize the interval modeling error, $\delta_{\text {model }}$. The coverage factor, $\mathrm{k}$, used to provide a given degree of confidence (ie 90\% assuming a uniform distribution, $\mathrm{k}=1.65$ ) [14]. The standard also outlines procedures to 
calculate numerical uncertainty, $\mathrm{u}_{\text {num }}$, the uncertainty in the simulated result from input parameters, $\mathrm{u}_{\text {input, }}$ and the experimental uncertainty, $\mathrm{u}_{\mathrm{D}}[14]$.

$$
\begin{gathered}
\delta_{\text {model }} \varepsilon\left[E-u_{\text {val }}, E+u_{\text {val }}\right] \\
E=S-D \\
u_{\text {val }}=k\left(\sqrt{u_{\text {num }}^{2}+u_{\text {input }}^{2}+u_{D}^{2}}\right)
\end{gathered}
$$

$\mathrm{U}_{\text {num }}$ is calculated using a Richardson's Extrapolation approach and defined as a five-step procedure [14].

Step 1, calculate representative grid size, $\boldsymbol{h}$ as shown in ( 4 ), ( 5 ), ( 6 ).

$$
\begin{gathered}
h_{1}=\left(\frac{\text { Total Volume }}{\text { total number of cells in fine grid }}\right)^{\frac{1}{3}} \\
h_{2}=\left(\frac{\text { Total Volume }}{\text { total number of cells in medium grid }}\right)^{\frac{1}{3}} \\
h_{3}=\left(\frac{\text { Total Volume }}{\text { total number of cells in coarse grid }}\right)^{\frac{1}{3}}
\end{gathered}
$$

Step 2 is to select three significantly $(r>1.3)$ grid sizes and computer the ratio as shown in equation ( 7 ), ( 8 ) [14]. 


$$
\begin{aligned}
& r_{21}=\frac{h_{2}}{h_{1}} \\
& r_{32}=\frac{h_{3}}{h_{2}}
\end{aligned}
$$

Step 3 is to calculate the observed order, p, as shown in equation ( 11 ) [14]. This equation must be solved iteratively.

$$
\begin{gathered}
\varepsilon_{21}=\mathrm{S}_{\mathrm{k} 2}-\mathrm{S}_{\mathrm{k} 1} \\
\varepsilon_{32}=\mathrm{S}_{\mathrm{k} 3}-\mathrm{S}_{\mathrm{k} 2} \\
p=\left[\frac{1}{\ln \left(r_{21}\right)}\right] *\left[\ln \left(\frac{\varepsilon_{32}}{\varepsilon_{21}}\right)+\ln \left(\frac{r_{21} p-\operatorname{sign}\left(\frac{\varepsilon_{32}}{\varepsilon_{21}}\right)}{r_{32} p-\operatorname{sign}\left(\frac{\varepsilon_{32}}{\varepsilon_{21}}\right)}\right)\right.
\end{gathered}
$$

Step 4 is to calculate the extrapolated values as shown in equation ( 12 ) [14].

$$
\begin{gathered}
S_{\text {ext }}^{21}=\frac{\left(r_{21}^{p} * S_{k 1}-S_{k 2}\right)}{\left(r_{21}^{p}-1\right)} \\
e_{a}{ }^{21}=\frac{\left(S_{k 1}-S_{k 2}\right)}{\left(S_{k 1}\right)}
\end{gathered}
$$

Step 5 is to calculate the fine grid convergence index and numerical uncertainty as shown in equation ( 14 ) [14]. This approached used a factor of safety of 1.25 and assumes the distribution is Gaussian about the fine grid, $95 \%$ confidence.

$$
\begin{aligned}
G C I_{\text {fine }}{ }^{21} & =\frac{1.25 * e_{a}^{21}}{\left(r_{21}{ }^{p}-1\right)} \\
U_{\text {num }} & =G C I_{\text {fine }}{ }^{21}
\end{aligned}
$$

$\mathrm{U}_{\text {input }}$ is calculated using a Taylor Series expansion in parameter space [14]. 


$$
u_{\text {input }}=\sqrt{\sum_{i=1}^{n}\left(\frac{\vartheta S}{\vartheta X_{i}} u_{x i}\right)^{2}}
$$

$\mathrm{U}_{\mathrm{D}}$ is calculated using test uncertainty methodology as defied in the standard [14]. The purpose of this paper is to show an estimate of numerical uncertainty without test data. The reader is referred to the ASME standard for further information.

\subsection{Proposed Methodology without Test Data}

There are a few items to note from the summary of the ASME standard. The summary assumes there are no random errors and none of the input variables are correlated. Additionally, the standard states the numerical error can be calculated by the 5-step procedure, which is essentially Richardson's Extrapolation Method. There are additional assumptions to Richardson's Extrapolation. To apply this method, the variable must be monotonically increasing or decreasing (ie in the extrapolated region). The input variables are assumed to be oscillatory convergence. A convergence study can be calculated to determine if the grid is monotonic, oscillatory, or divergence.

Convergence studies require a minimum of three solutions to evaluate convergence with respect to an input parameter [8]. Consider the situation for 3 solutions corresponding to fine $\mathrm{S}_{\mathrm{k} 1}$, medium $S_{\mathrm{k} 2}$, and coarse $S_{\mathrm{k} 3}$ values for the $k$ th input parameter [8]. Solution changes $\boldsymbol{\varepsilon}$ for mediumfine and coarse-medium solutions and their ratio $\mathrm{R}_{\mathrm{k}}$ are defined by [8]:

$$
\varepsilon_{21}=S_{k 2}-S_{k 1}
$$




$$
\begin{gathered}
\varepsilon_{32}=S_{k 3}-S_{k 2} \\
R_{k}=\varepsilon_{21} / \varepsilon_{32}
\end{gathered}
$$

Three convergence conditions are possible [8]:

(i) Monotonic convergence: $0<\mathrm{R}_{\mathrm{k}}<1$

(ii) Oscillatory convergence: $\mathrm{R}_{\mathrm{k}}<0^{\mathrm{i}}$

(iii) Divergence: $\mathrm{R}_{\mathrm{k}}>1$

The methodology outlined in ASME V\&V-2009 [14] assumes monotonic convergence criteria for $\mathrm{u}_{\text {num. }}$ Further increasing the grid does not always provide a monotonically increasing result. This is shown in AIAA-2013-0258 [19]. The proposed methodology is to treat all input parameters including the grid as an oscillatory convergence study. The uncertainty for cells with oscillatory convergence, using the following method outlined by Stern, Wilson, Coleman, and Paterson [8], can be calculated as follows in equation ( 20$)$. $S$ is the simulated result. For this case it is the upper velocity $\boldsymbol{S}_{U}$ and the lower velocity $\boldsymbol{S}_{\boldsymbol{L}}$.

$$
U_{\text {Oscillatory }}=\left|\frac{1}{2}\left(S_{U}-S_{L}\right)\right|
$$

The proposed methodology as compared to the ASME Standard is as follows. If there is no experimental data, $\mathrm{D}=0, \delta_{\mathrm{D}}=0$, and $\mathrm{u}_{\mathrm{D}}=0$.

$$
\begin{gathered}
E=S-D=S \\
\delta S=S-T \\
E=S-D=T+\delta S-\left(T+\delta_{D}\right)=\delta_{S}-\delta_{D}=\delta_{S} \\
u_{\text {val }}=k\left(\sqrt{u_{\text {num }}^{2}+u_{\text {input }}^{2}+u_{D}^{2}}\right)=u_{\text {val }}=k\left(\sqrt{u_{\text {num }}^{2}+u_{\text {input }}^{2}}\right)
\end{gathered}
$$


Report the simulated result, $\mathrm{S}$ as

$$
S \stackrel{+}{-} u_{v a l}
$$

Also instead of assuming a gauss-normal distribution as in the standard when including test data, the k-value will come from the Student-T distribution as shown in Table 1. The Student-T distribution is used for experimental uncertainty calculations for a limited number of samples [20].

The quantity of interest for the ECS / spacecraft system is velocity magnitude. Three grids can be compared, and the convergence conditions determined for every point in the computational domain. This is accomplished through interpolation between the medium to coarse grid and the fine to coarse grid. The velocity magnitude from the medium and fine grids are interpolated on to the coarse grid. Then the solutions changes, $\mathbf{\varepsilon 2 1}, \mathbf{\varepsilon} \mathbf{3 2}, \mathbf{R}_{\mathbf{k}}$, and convergence conditions are calculated for every point in the domain.

This interpolation can induce errors in the solution. The method used in the backward facing step used a 'zeroth' order interpolation scheme in FLUENT. Section 5.1 describes an approach to find a higher order interpolation scheme and plot the three different convergence conditions. Treating the grid as a monotonically increasing parameter in the entire domain may be inappropriate. Additionally for an oscillatory convergence parameter, Stern, Wilson, Coleman, and Paterson recommend equation 12.

It will be shown in section 5.2 that treating the grid as an oscillatory input parameter provides an alternative method to estimate the uncertainty in the numeric's. 
Table 1: Student T Distribution, k Values [20]

\begin{tabular}{|c|c|c|}
\hline $\begin{array}{l}\text { Number of } \\
\text { Cases }\end{array}$ & $\begin{array}{c}\text { Degrees of } \\
\text { Freedom }\end{array}$ & $\begin{array}{c}\text { Confidence } \\
90 \%\end{array}$ \\
\hline 2 & 1 & 6.314 \\
\hline 3 & 2 & 2.92 \\
\hline 4 & 3 & 2.353 \\
\hline 5 & 4 & 2.132 \\
\hline 6 & 5 & 2.015 \\
\hline 7 & 6 & 1.943 \\
\hline 8 & 7 & 1.895 \\
\hline 9 & 8 & 1.86 \\
\hline 10 & 9 & 1.833 \\
\hline 11 & 10 & 1.812 \\
\hline 12 & 11 & 1.796 \\
\hline 13 & 12 & 1.782 \\
\hline 14 & 13 & 1.771 \\
\hline 15 & 14 & 1.761 \\
\hline 16 & 15 & 1.753 \\
\hline 17 & 16 & 1.746 \\
\hline 18 & 17 & 1.74 \\
\hline 19 & 18 & 1.734 \\
\hline 20 & 19 & 1.729 \\
\hline 21 & 20 & 1.725 \\
\hline 22 & 21 & 1.721 \\
\hline 23 & 22 & 1.717 \\
\hline 24 & 23 & 1.714 \\
\hline 25 & 24 & 1.711 \\
\hline 26 & 25 & 1.708 \\
\hline 27 & 26 & 1.706 \\
\hline 28 & 27 & 1.703 \\
\hline 29 & 28 & 1.701 \\
\hline 30 & 29 & 1.699 \\
\hline 31 & 30 & 1.697 \\
\hline 41 & 40 & 1.684 \\
\hline 51 & 50 & 1.676 \\
\hline 61 & 60 & 1.671 \\
\hline 81 & 80 & 1.664 \\
\hline 101 & 100 & 1.66 \\
\hline 121 & 120 & 1.658 \\
\hline infty & infty & 1.645 \\
\hline
\end{tabular}




\section{CHAPTER THREE: APPLYING THE "STATE OF THE ART" CFD UNCERTAINTY ANALYSIS TO A BACKWARD FACING STEP}

\subsection{Grid Refinement Study and Velocity Prediction}

The author applied the ASME standard to a backward facing step in AIAA-2013-0258 [19]. A summary of this paper is included here.

The quantity of interest for the backward facing setup is velocity magnitude. Three grids were compared, and the convergence conditions were determined for every point in the computational domain. This is accomplished through interpolation between the medium to coarse grid and the fine to coarse grid. The velocity magnitude from the medium and fine grids are interpolated on to the coarse grid. Then the solutions changes, $\mathbf{\varepsilon 2 1}, \mathbf{\varepsilon 3 2}, \mathbf{R}_{\mathbf{k}}$, and convergence conditions are calculated for every point in the domain. Figure 1 shows the different convergence conditions inside the computational domain for the grid refinement study.

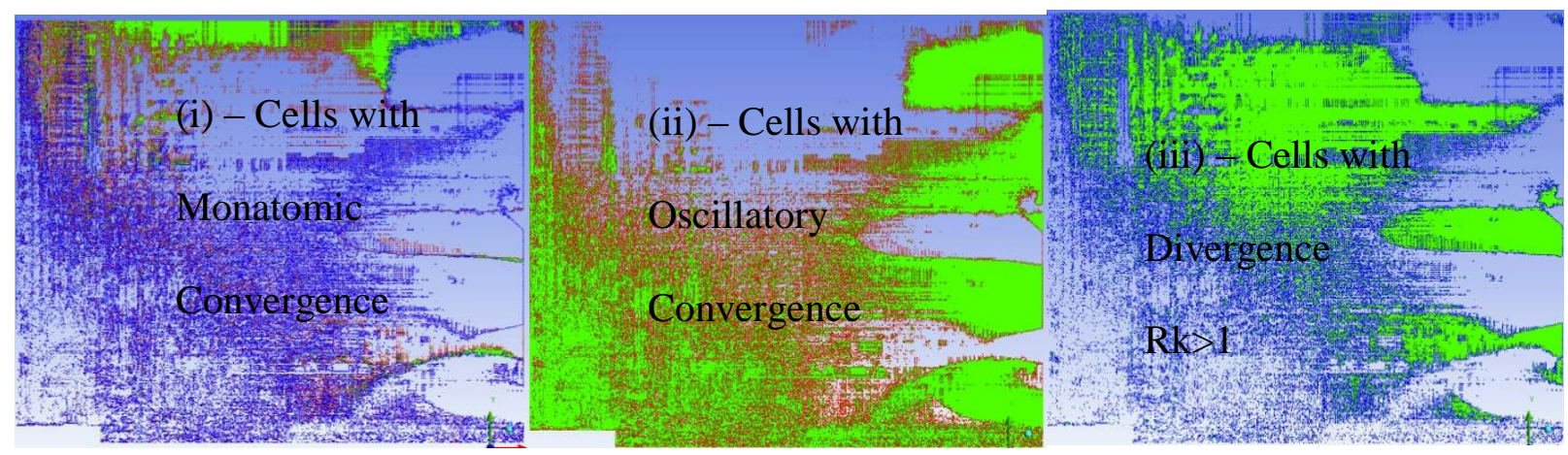

Figure 1: Convergence conditions for the backward facing step - Grid refinement 


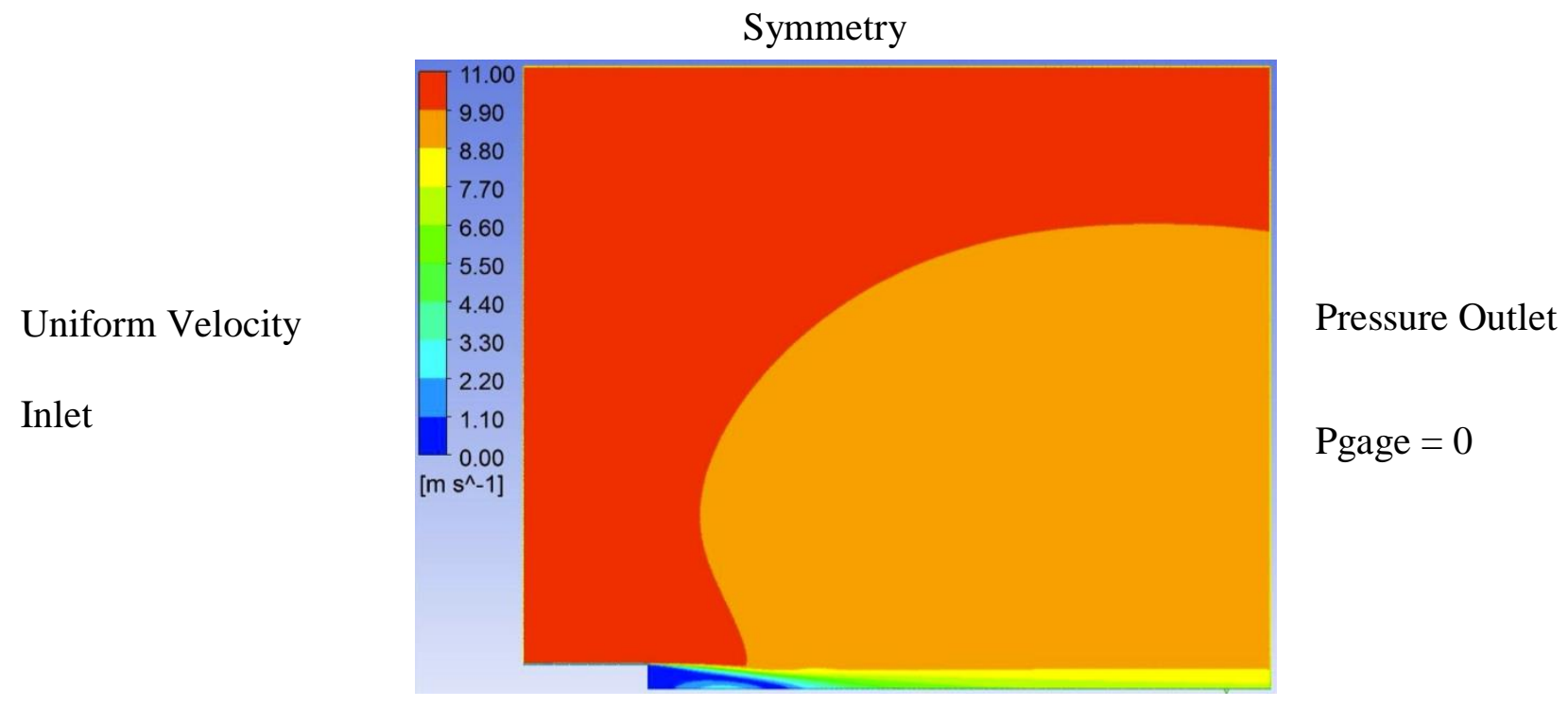

Figure 2: Velocity Magnitude for Flow over a Backward Facing Step - 1,192,000 cells

\subsection{CFD Uncertainty Analysis of Backward Facing Step}

The following input variables were considered for the uncertainty analysis.

Input Variables:

$$
U_{C F D}=\left(\sum_{i=1}^{J}\left\{\left(\frac{\partial r}{\partial X_{i}}\right)^{2} B_{i}^{2}\right\}\right)^{1 / 2}
$$

A list of variables for the k-e-realizable turbulence model analyzed is listed in Table 2. Expanding the data reduction equation for the listed variables as shown in order from top to bottom. 


$$
\begin{aligned}
U_{C F D-\text { Velocity }} & =\left(\left(\left(\frac{\partial V}{\partial e}\right)^{2} B_{e}^{2}\right)+\left(\left(\frac{\partial V}{\partial k}\right)^{2} B_{k}^{2}\right)+\left(\left(\frac{\partial V}{\partial p}\right)^{2} B_{p}^{2}\right)\right. \\
& +\left(\left(\frac{\partial V}{\partial U}\right)^{2} B_{u}^{2}\right)+\left(\left(\frac{\partial V}{\partial n u}\right)^{2} B_{\text {nu }}^{2}\right)+\left(\left(\frac{\partial V}{\partial g}\right)^{2} B_{g}^{2}\right) \\
& +\left(\left(\frac{\partial V}{\partial n u m}\right)^{2} B_{\text {num }}^{2}\right)+\left(\left(\frac{\partial V}{\partial \text { solver }}\right)^{2} B_{\text {solver }}^{2}\right) \\
& \left.+\left(\left(\frac{\partial V}{\partial t u r b}\right)^{2} B_{\text {turb }}^{2}\right)\right)^{1 / 2}
\end{aligned}
$$

\begin{tabular}{|c|c|c|c|}
\hline $\begin{array}{c}\text { Type of } \\
\text { Variable }\end{array}$ & Variables Xi & Value & $\begin{array}{c}\text { Bias } \\
\text { Error }\end{array}$ \\
\hline $\begin{array}{l}\text { Boundary } \\
\text { Conditions }\end{array}$ & $\begin{array}{l}\text { epsilion turbulent mixing length dissipation rate } \\
\text { inlet }(\mathrm{m} 2 / \mathrm{s} 3)\end{array}$ & 0.5 & 0.5 \\
\hline & $\mathrm{k}$ turbulent intensity kinetic energy inlet $(\mathrm{m} 2 / \mathrm{s} 2)$ & 0.05 & 0.05 \\
\hline & pressure outlet $(\mathrm{Pa})$ & 101325 & $2 \%$ \\
\hline & velocity inlet $(\mathrm{m} / \mathrm{s})$ & 10 & 0.5 \\
\hline $\begin{array}{c}\text { Fluid } \\
\text { Properties }\end{array}$ & $\begin{array}{l}\text { kinematic viscosity nu represents air [0-50-100] } \\
\text { deg C }\end{array}$ & $1.79 \mathrm{E}-06$ & $\begin{array}{l}{[13.6 \mathrm{e}-06} \\
->23.06 \mathrm{e}- \\
06]\end{array}$ \\
\hline Grid Size & Method - Uses Oscillatory Uncertainty & \multirow[t]{2}{*}{$\begin{array}{l}1,192,000 \\
1,862,500 \\
3,311,689\end{array}$} & \\
\hline Numerical & $\begin{array}{l}\text { Method - Uses Richardson's Extrapolation (ASME } \\
5 \text { Step Procedure) - Calculated for Velocity at each } \\
\text { Cell } \\
\end{array}$ & & \\
\hline Solver & OpenFOAM (SimpleFoam) vs. Fluent & & \\
\hline $\begin{array}{c}\text { Turbulence } \\
\text { Models }\end{array}$ & ke-realiable, kwSST, and SpalartAllmaras & & \\
\hline
\end{tabular}

Table 2: Uncertainty Variables, Xi - Backward Facing Step 


\section{3 $\underline{\text { Results and Discussion }}$}

Each of the variables was analyzed separately for their elemental error sources. The following plots show the each variables and their corresponding uncertainty plot as a function of the percent uncertainty in the CFD Velocity prediction. The percent uncertainty is calculated by dividing by the local velocity (ie the uncertainty velocity in each cell divided by the velocity in each cell). There may be a more appropriate way to non-dimensionalize, such as using the average inlet velocity.

The uncertainty for each of the following was calculated as shown below for each cell using the following method outlined by Stern, Wilson, Coleman, and Paterson [8]. $S$ is the simulated result. For this case it is the upper velocity $\boldsymbol{S}_{U}$ and the lower velocity $\boldsymbol{S}_{\boldsymbol{L}}$.

$$
U_{\text {Oscillatory }}=\frac{1}{2}\left(S_{U}-S_{L}\right)
$$

For the epsilon turbulent mixing dissipation rate of the inlet a value of $0.5+/-0.5 \mathrm{~m}^{2} / \mathrm{s}^{3}$ was used. The uncertainty in the velocity prediction was $0-1.155$ percent as shown in Figure 3.

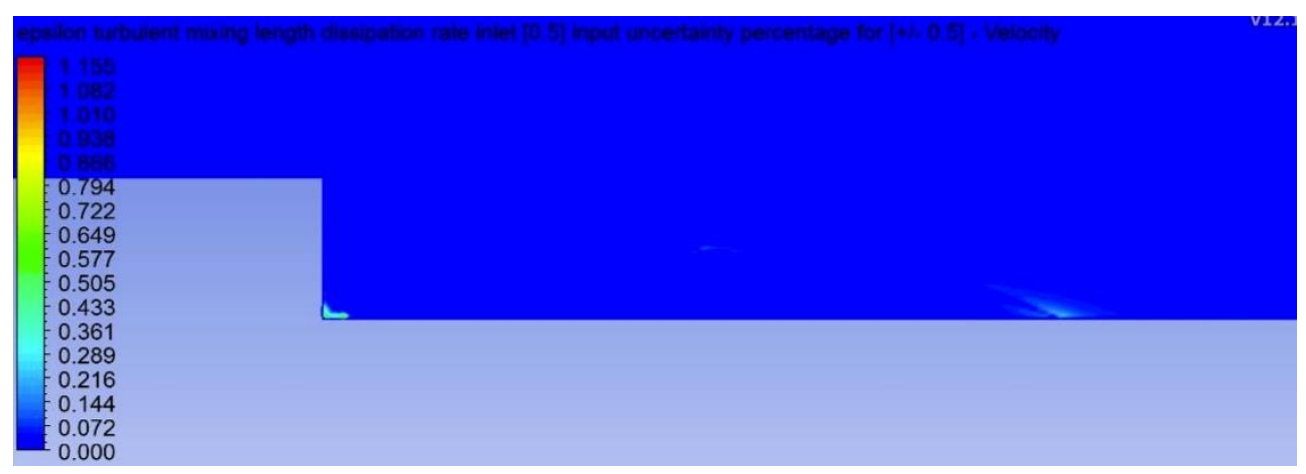

Figure 3: Epsilon Turbulent Mixing Length Dissipation Rate Inlet - Velocity Uncertainty Percentage 
For the turbulent intensity kinetic energy of the inlet a value of $0.05+/-0.05 \mathrm{~m}^{2} / \mathrm{s}^{2}$ was used. The uncertainty in the velocity prediction was $0-0.785$ percent as shown in Figure 4.

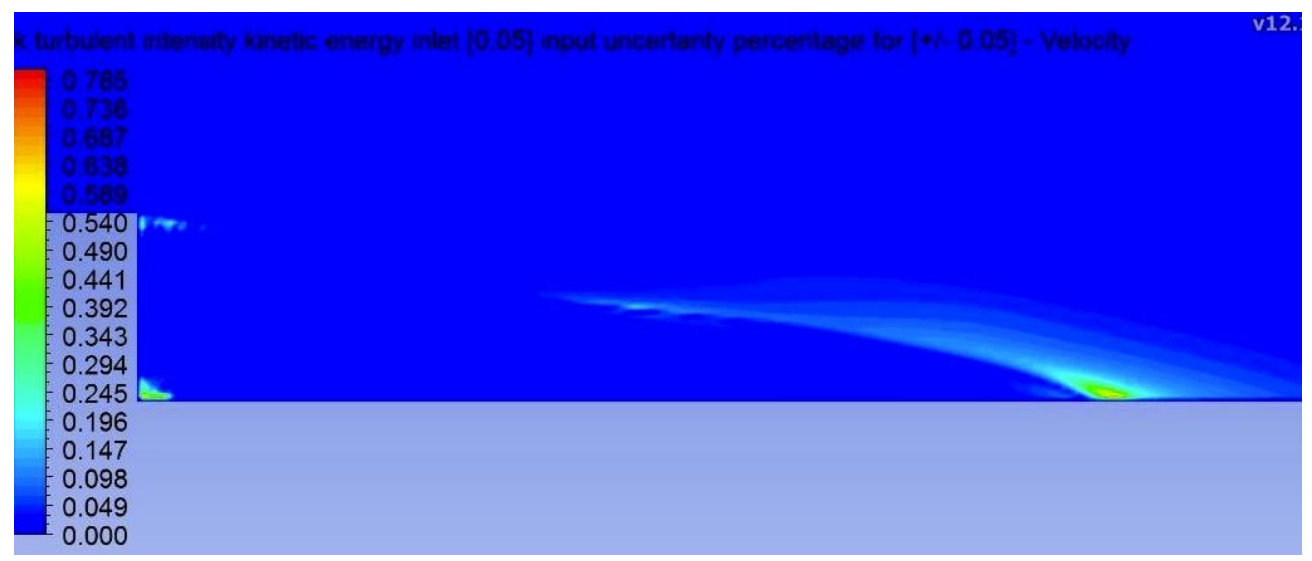

Figure 4: kTurbulent Intensity Kinetic Energy Inlet - Velocity Uncertainty Percentage

For the pressure outlet a value of $101325+/-2 \%$ Pa was used and the uncertainty in the velocity prediction was $0-20$ percent as shown in Figure 5.

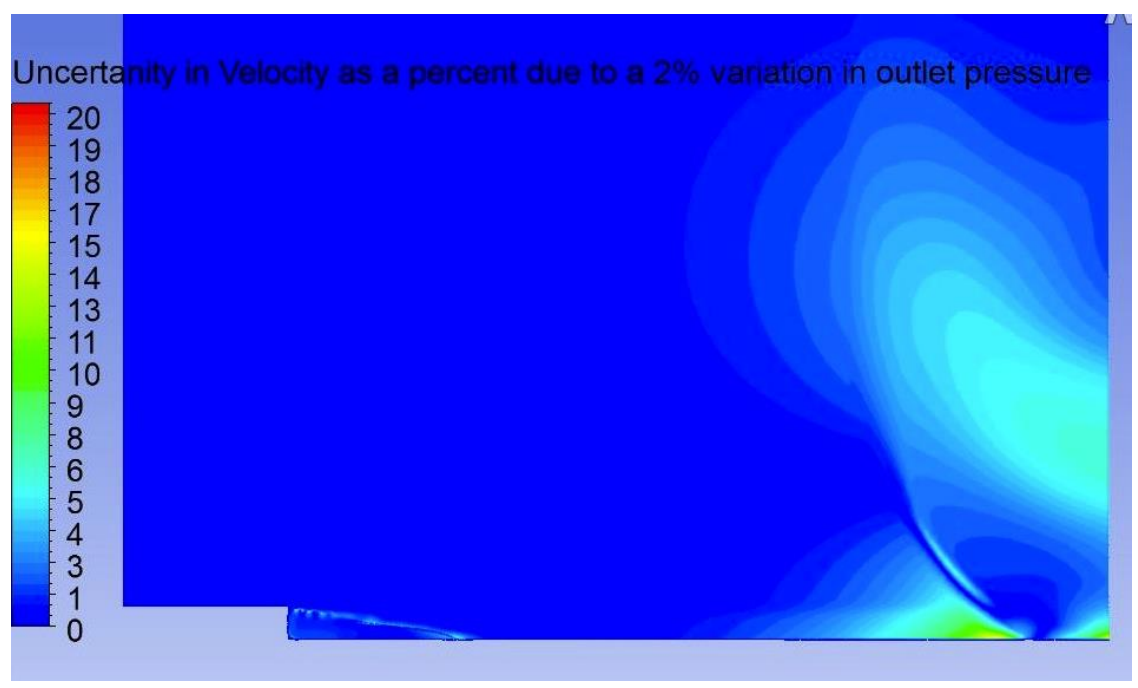

Figure 5: Pressure Outlet - Velocity Uncertainty Percentage

For the inlet velocity a value of $10+/-0.5 \mathrm{~m} / \mathrm{s}$ was used and the uncertainty in the velocity prediction was $0-6.558$ percent as shown in Figure 6 . 


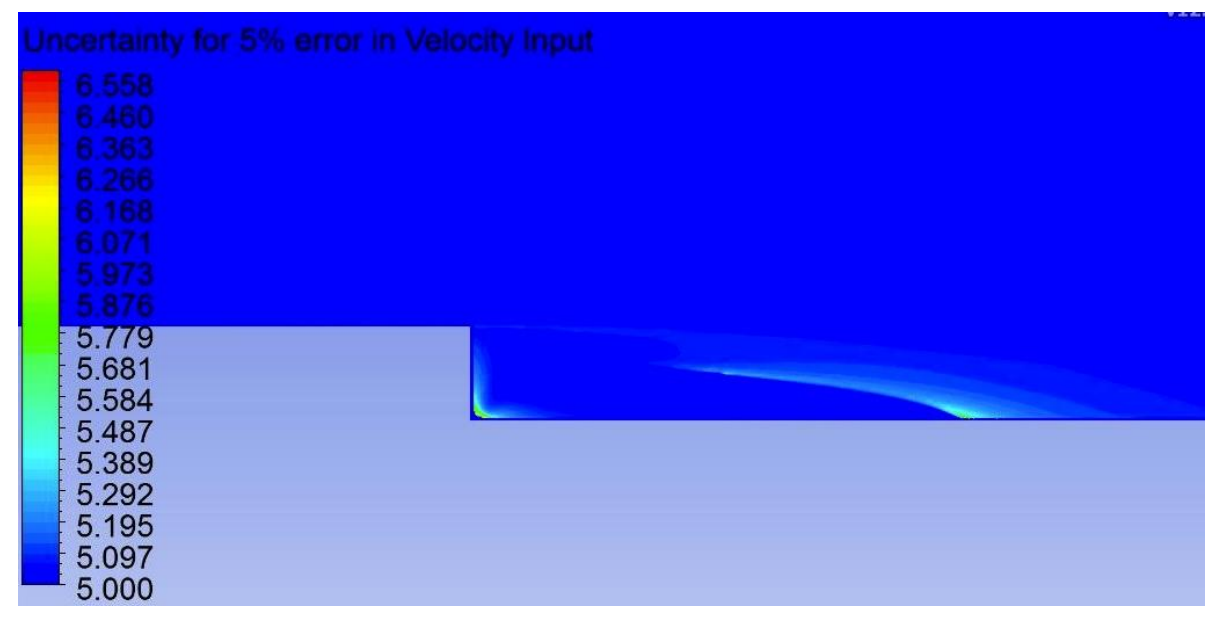

Figure 6: Velocity Inlet - Velocity Uncertainty Percentage

For the kinematic viscosity of air a value of nu=17.06e-06 [13.6e-06 -> 23.06e-06] $\left(\mathrm{m}^{2} / \mathrm{s}\right)$ was chosen to represent air from 0 to 100 degrees Celsius. The uncertainty in the velocity prediction was $0-27.727$ percent as shown in Figure 7 .

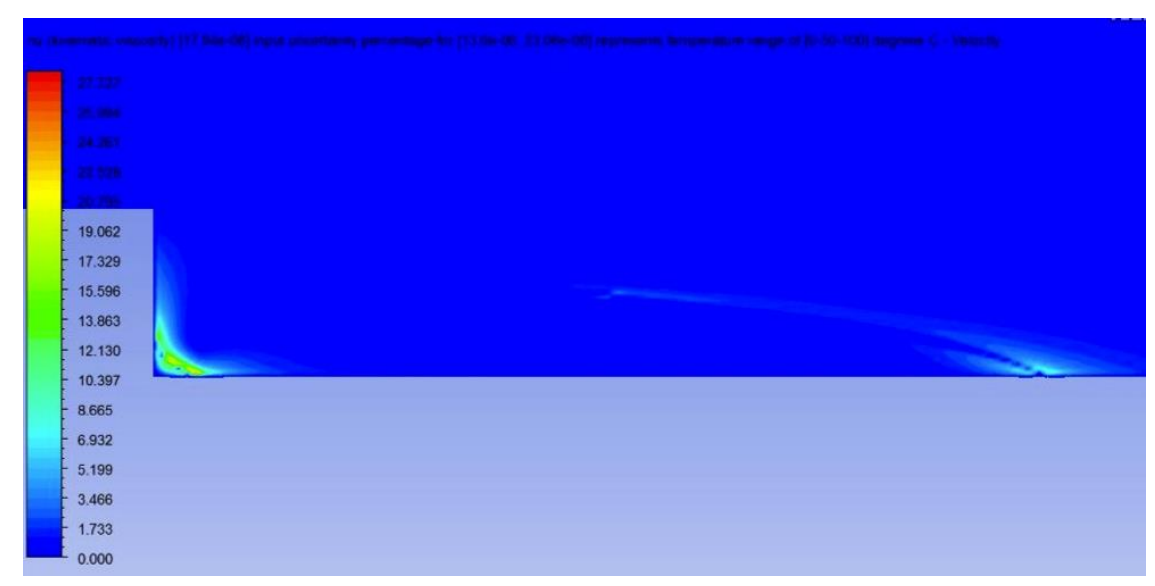

Figure 7: Kinematic Viscosity - Velocity Uncertainty Percentage

For a grid size of 1,192,000 cells [grid $2-1,862,500$ cells], [grid3 - 3,311,689 cells], the uncertainty in the velocity prediction was $0-698$ percent as shown in Figure 8. 


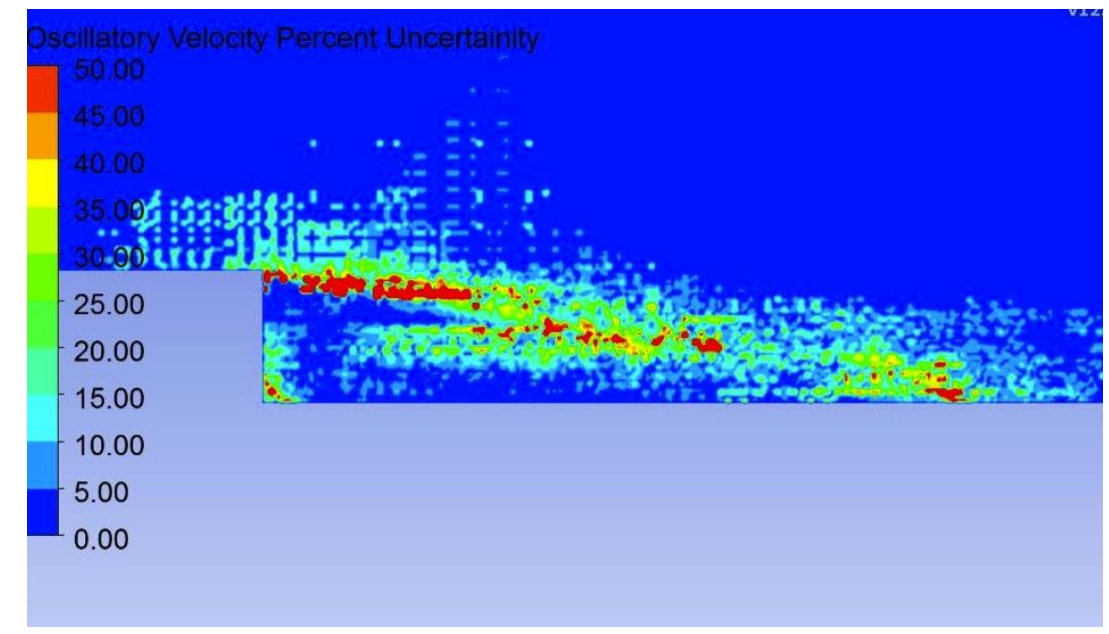

Figure 8: Grid Size - Velocity Uncertainty Percentage

The ke-realiable, kwSST, and SpalartAllmaras turbulence models converged using OpenFoam and the uncertainty was calculated as an oscillatory input parameter as shown in Figure 9.

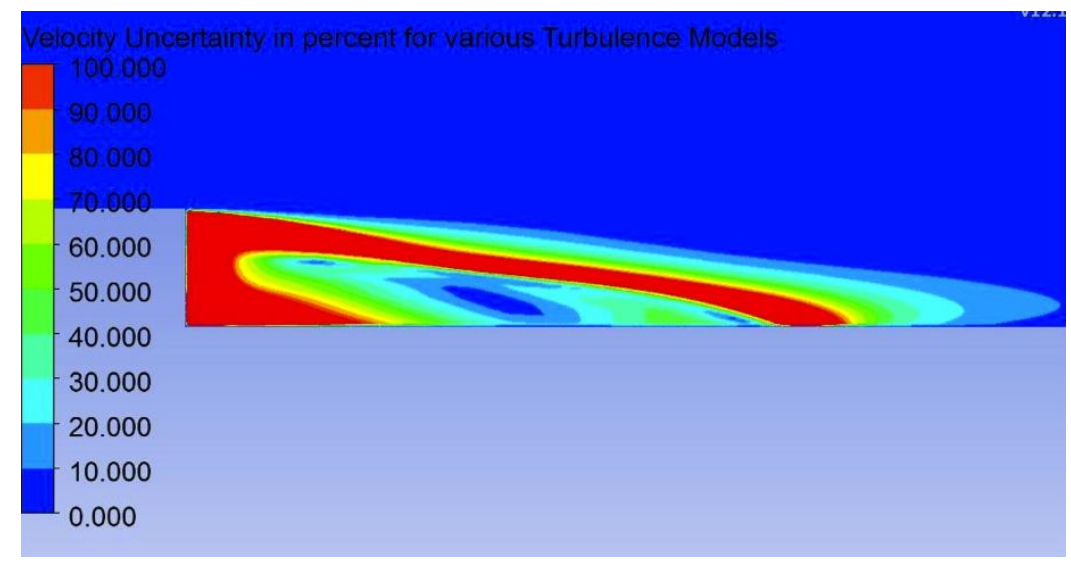

Figure 9: Turbulence Models - Velocity Uncertainty Percentage

OpenFoam and Fluent were used as the solvers to calculate the velocity distribution on the backward facing step and the uncertainty was calculated as an oscillatory input parameter as shown in Figure 10. 


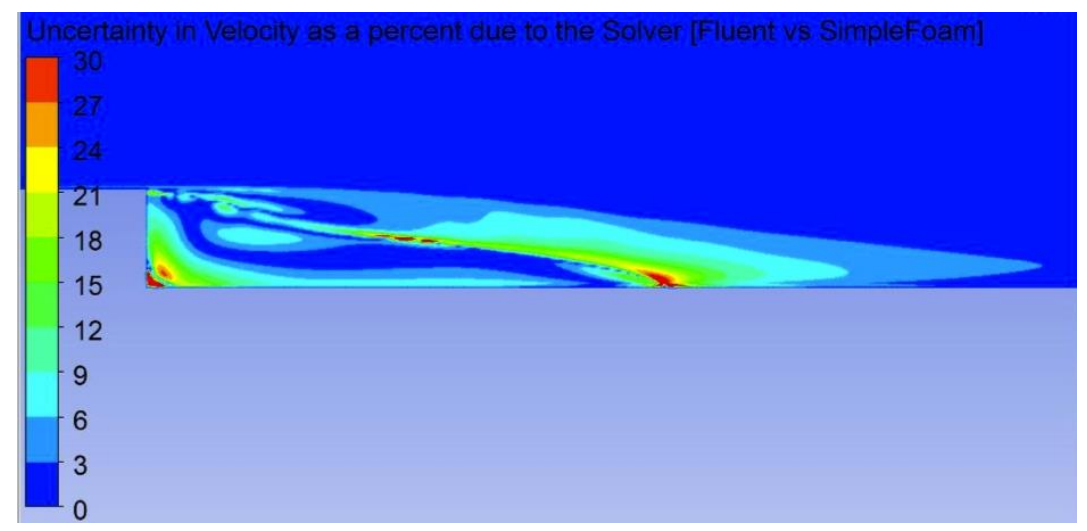

Figure 10: Solver - Velocity Uncertainty Percentage

The uncertainties of the variables with monotonic convergence (numerical) are calculated using Richardson's extrapolation as outlines by ASME V\&V-2009 [14]. This is accomplished through the five-step procedure described in equations ( 4 ), ( 5 ), ( 6 ), ( 7 ), ( 8 ), ( 9 ), ( 10 ), ( 11 )$,(12),(13),(14)$.

For a grid size of 1,192,000 cells [grid 2 -1,862,500 cells], [grid3 - 3,311,689 cells], the uncertainty in the velocity prediction was $0-5300$ percent as shown in Figure 11 as estimated by Richardson's extrapolation method.

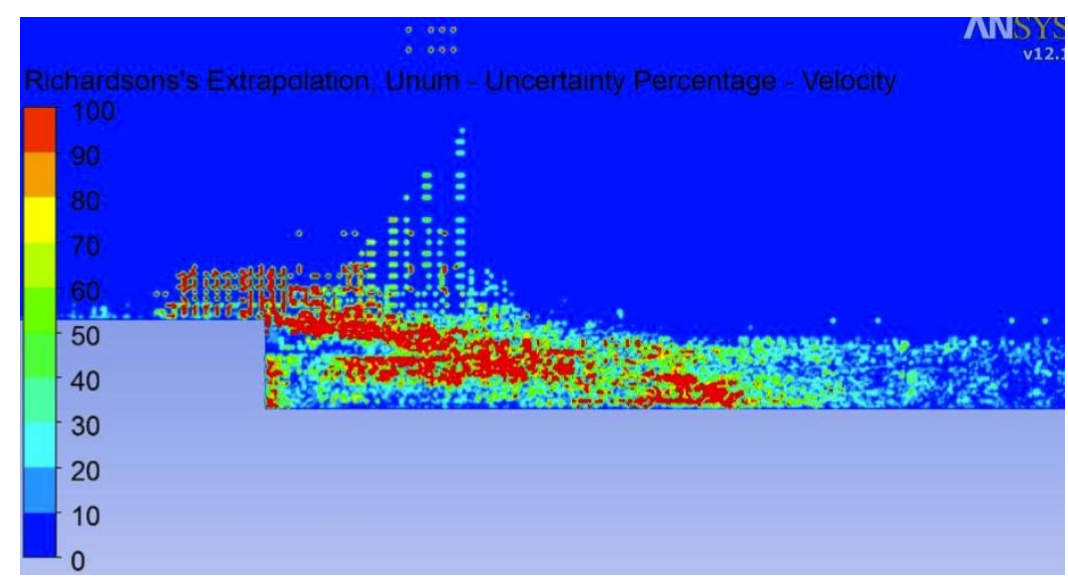

Figure 11: Numerical - Velocity Uncertainty Percentage 
A root-sum-squared (rss) of the uncertainty variables was calculated (omitting Richardson's Extrapolation) and the velocity magnitude is shown in Figure 12 with the corresponding uncertainty.
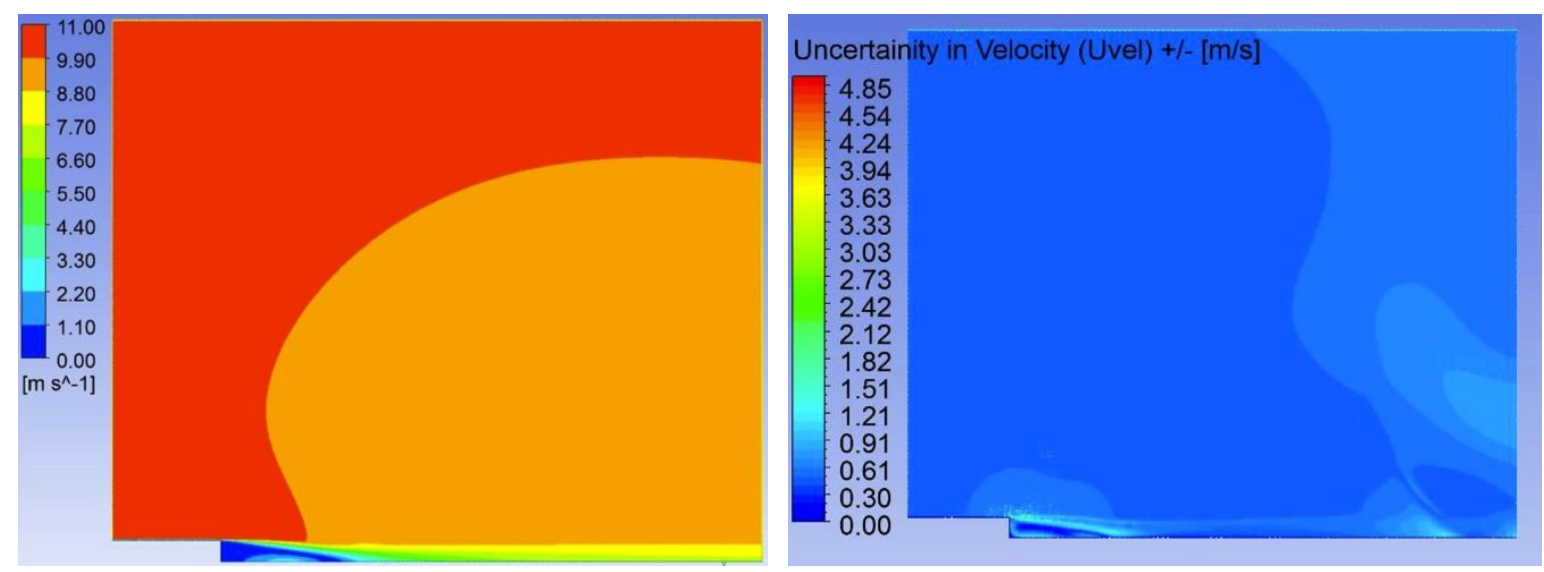

Figure 12: Velocity Prediction and Uncertainty Plot for ke-realizable Turbulence Model

The highest uncertainty is $+/-4.85 \mathrm{~m} / \mathrm{s}$. This occurs in the region shown in Figure 14 in red. Figure 14 is the same data presented on the right hand side of Figure 13, except zoomed in to the region near the backward step and a smaller scale is used.

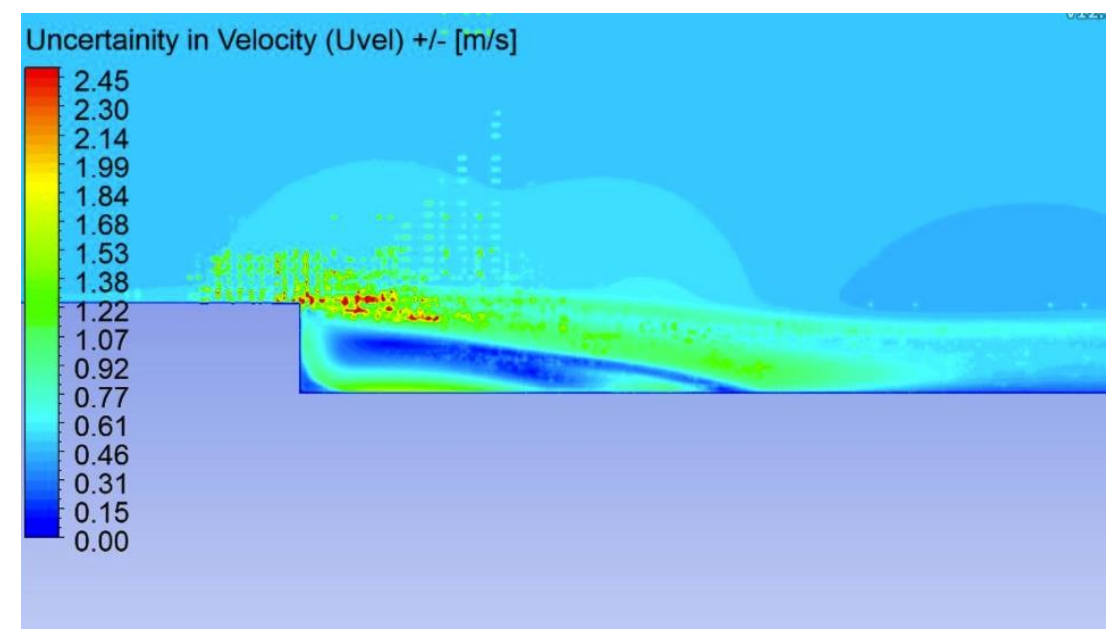

Figure 13: Velocity Uncertainty Plot for ke-realiable Turbulence Model 
The monotonic convergence uncertainty calculation was omitted in the rss uncertainty plot due to the values produced by using this method. The method produced uncertainty values on the order of 5000 percent of the localized velocity in the region near the backward step. It is believed this is due to the turbulence and/or the interpolation between the 3 grids. Turbulence is calculated as a steady state value and fluctuations about the steady state. The fluctuations are inducing a nonlinear result between the three grids and providing very large uncertainty bands in the localized region near the backward step. However, once you move approximately 5 lengths downstream of the backward step, the method begins producing reasonable results of $0-30$ percent of the localized velocity. Treating the highly turbulent region behind the backward step as a monotonic case is inappropriate. It is believed that treating the grid as an input parameter with oscillatory convergence provides better results for a steady state, turbulent CFD simulation. This is evident in the $R_{k}$ values shown in Figure 1. Most of the cells are exhibiting oscillatory convergence. It is believed all cells are exhibiting oscillatory convergence, however depending on when the sample takes place, one could misrule the results as monotonic or divergent. The interpolation between the three grids could also be inducing this non-linear result. The current method for interpolation is using FLUENT to write out an interpolation file, then reading the file back into FLUENT onto a different grid. This method has been evaluated in section 5.1 and other interpolations methods considered. 


\section{CHAPTER FOUR: SPACECRAFT ECS SYSTEM OVERVIEW AND MODELING}

\subsection{Spacecraft ECS System Overview}

The author published the work in this chapter as AIAA-2014-0440 [21]. Prior to launch, cold air (air conditioning) flows downward around the spacecraft after it has been encapsulated in the Payload Fairing [2]. The cold air is delivered through an air-conditioning (AC) pipe, which intersects the fairing and flows past a diffuser located at the pipe/fairing interface [2]. After passing over the spacecraft, it is finally discharged through vents [2]. The Payload Fairing air conditioning is cut off at lift off [2]. An overview of the geometry for an Environmental Control System (ECS) along with the swirled airflow is shown in Figure 14 and Figure 15, respectively.

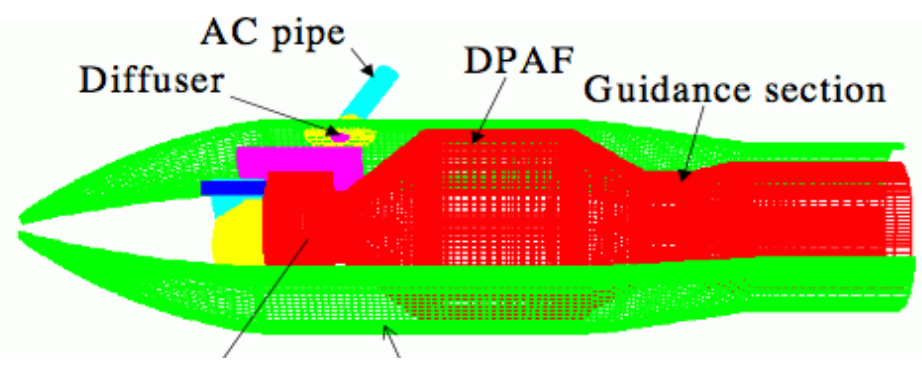

Figure 14: Environmental Control System (ECS) Overview [22]

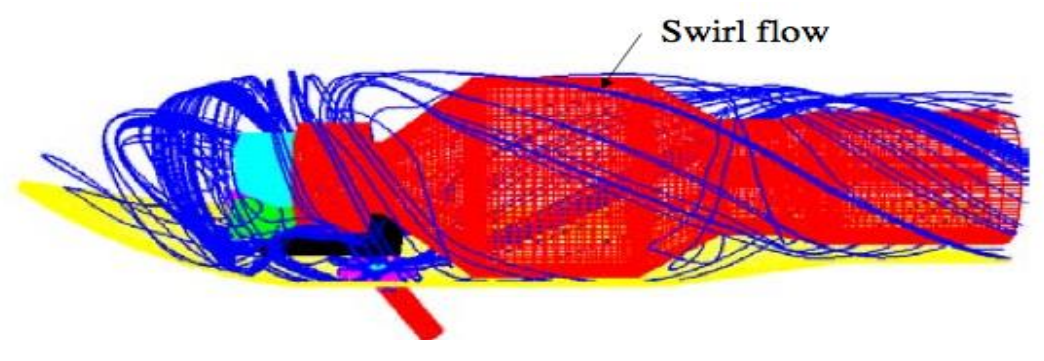

Figure 15: Environmental Control System (ECS) Airflow Swirl [22] 
This problem has been previously solved using overset grids and compared to laser doppler test data as described in AIAA-2005-4910 [23]. An example of the airflow testing performed is shown in Figure 16.
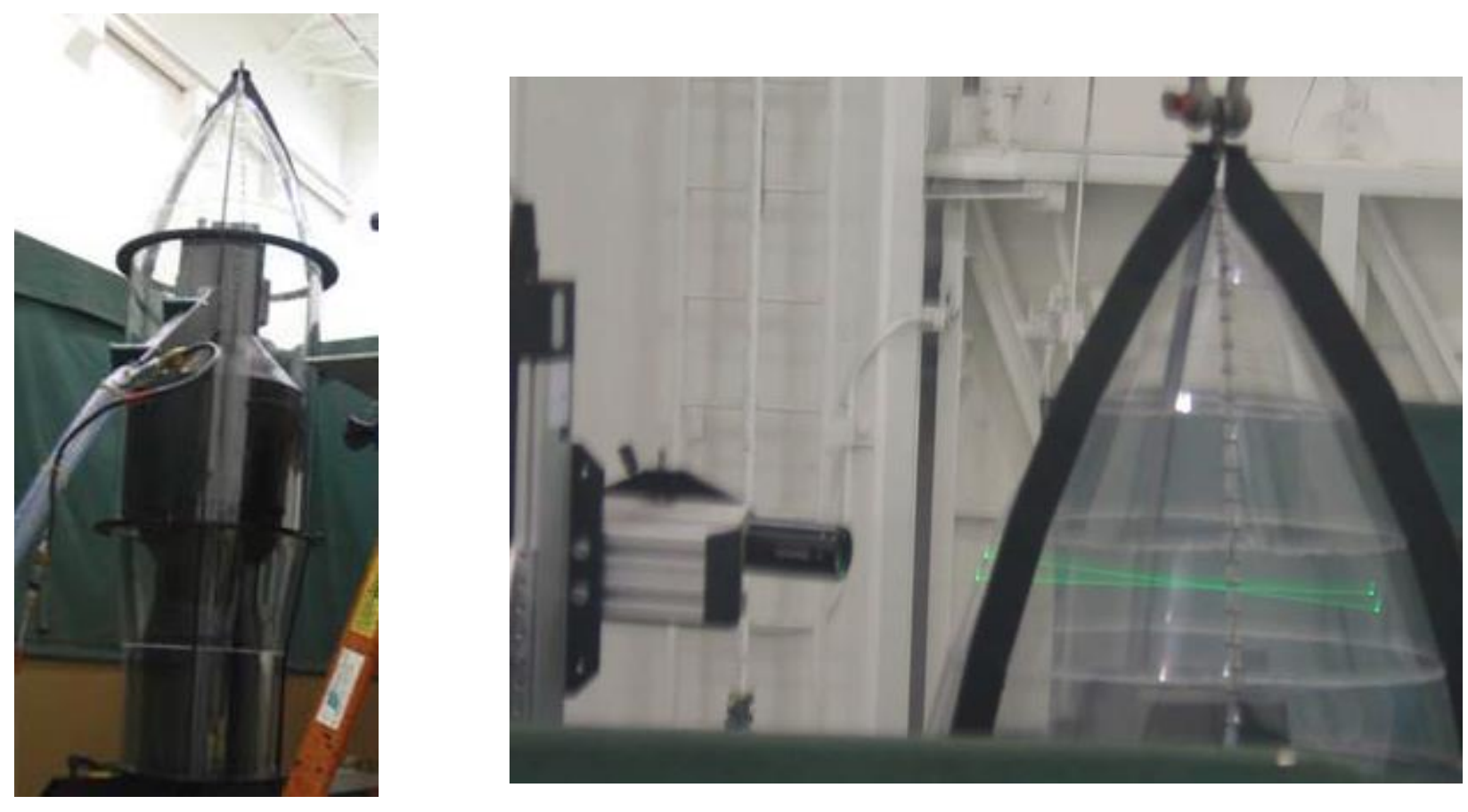

Figure 16: Environmental Control System (ECS) Airflow Testing [24]

The example shown above is the only published result of the ECS airflow problem. It is difficult to publish this material due to the proprietary information needed. There are seven different rockets currently being used in the United States for Evolved Expendable Launch Vehicles (EELV). These rockets include the Delta II, Delta IV, Atlas V, Pegasus, Taurus, and Falcon 9 [25]. A summary of each of these rockets's ECS systems which are available in the public information is included below. To investigate the problem (3) generic representations were created which encompass the flow regimes seen in the EELV fleet. Each of these rockets has a publicly available source called a payload planners guide or users guide. Each of these guides has 
been studied extensively and the appropriate information related to the ECS systems are presented next.

\subsubsection{Publically Available Information on EELV ECS Systems}

For the Delta II vehicle, air-conditioning is supplied to the spacecraft via an umbilical after the payload fairing is mated to the launch vehicle [26]. The payload air-distribution system provides air at the required temperature, relative humidity, and flow rate as measured [26]. The airdistribution system uses a diffuser on the inlet air-conditioning duct at the fairing interface [26]. If required, a deflector can be installed on the inlet to direct the airflow away from sensitive spacecraft components [26]. The air can be supplied to the payload between a rate of 1300 to 1700 scfm [26]. The diameter of Fairing is 3 meters [26].

For the Delta IV, the air is supplied to the payload at a maximum flow rate of $36.3 \mathrm{~kg} / \mathrm{min}$ to $72.6 \mathrm{~kg} / \mathrm{min}$ (80 to $160 \mathrm{lb} / \mathrm{min}$ ) for $4-\mathrm{m}$ fairing launch vehicles and $90.7 \mathrm{~kg} / \mathrm{min}$ to $136.0 \mathrm{~kg} / \mathrm{min}$ (200 to $300 \mathrm{lb} / \mathrm{min}$ ) for 5-m fairing launch vehicles [27]. Air flows around the payload and is discharged through vents in the aft end of the fairing [27]. Fairing sizes are 4 meters and 5 meters in diameter [27].

For the Atlas V, internal ducting defectors in the PLF direct the gas upward to prevent direct impingement on the spacecraft [28]. The conditioning gas is vented to the atmosphere through one-way flapper doors below the spacecraft [28]. The PLF air distribution system will provide a maximum air flow velocity in all directions of no more than $9.75 \mathrm{mps}$ (32 fps) for the Atlas V 400 and $10.67 \mathrm{mps}$ (35 fps) for the Atlas V 500 [28]. There will be localized areas of higher flow 
velocity at, near, or associated with the air conditioning outlet [28]. Maximum air flow velocities correspond to maximum inlet mass flow rates [28]. Reduced flow velocities are achievable using lower inlet mass flow rates [28].

- Flow Rates
A) Atlas V 400: $0.38-1.21 \mathrm{~kg} / \mathrm{s} \pm 0.038 \mathrm{~kg} / \mathrm{s}(50-160 \mathrm{lb} / \mathrm{min} \pm 5 \mathrm{lb} / \mathrm{min})$, [28]
B) Atlas V 500: $0.38-2.27 \mathrm{~kg} / \mathrm{s} \pm 0.095 \mathrm{~kg} / \mathrm{s}(50-300 \mathrm{lb} / \mathrm{min} \pm 12.5 \mathrm{lb} / \mathrm{min})$ [28]

The fairing sizes are 4meters and 5 meters in diameter [28].

For the Pegasus vehicle, the fairing is continuously purged with filtered air [29]. The flowrate of air through the fairing is maintained between 50 and $200 \mathrm{cfm}$ [29]. The air flow enters the fairing forward of the payload and exits aft of the payload [29]. There are baffles on the inlet that minimize the impingement velocity of the air on the payload [29]. The fairing diameter is 0.97 meters [29].

For the Taurus vehicle, upon encapsulation within the fairing and for the remainder of ground operations, the payload environment will be maintained by the Taurus Environmental Control System (ECS) [30]. The fairing inlet conditions are selected by the Customer [30]. The fairing diameters are 63 inches and 92 inches [30].

For the Falcon 9 vehicle, once fully encapsulated and horizontal, the Environmental Control System (ECS) is connected [31]. Payload environments during various processing phases are [31]:

- In hanger, encapsulated - Flow Rate: 1,000 cfm [31]

- During rollout: 1,000 cfm [31] 
- On pad: Variable from 1000 to $4500 \mathrm{cfm}[31]$

The fairing diameter is 5.2 meters [31].

4.1.2 Modeling and CFD Analysis of (3) Generic Non-Proprietary Environmental Control System and Spacecraft Configurations

The following information can be concluded about the publically releasable ECS system data presented in the previous section. The fairing sizes are approximately $1 \mathrm{~m}, 1.6 \mathrm{~m}, 2.3 \mathrm{~m}, 3 \mathrm{~m}, 4 \mathrm{~m}$, $5 \mathrm{~m}$ in diameter. The following (3) generic fairing diameters are selected to envelop the EELV fairing configurations as follows.

$$
\begin{aligned}
& -0.75 \mathrm{~m} \\
& -\quad 3.5 \mathrm{~m} \\
& -\quad 5.5 \mathrm{~m}
\end{aligned}
$$

The inlet conditions range from $1000 \mathrm{cfm}$ to $4500 \mathrm{cfm}$.

The three proposed generic models have been created via Computer Aided Drafting (CAD) model software Pro/ENGINEER. The configurations are shown in Figure 17, Figure 18, and Figure 19, respectively. 

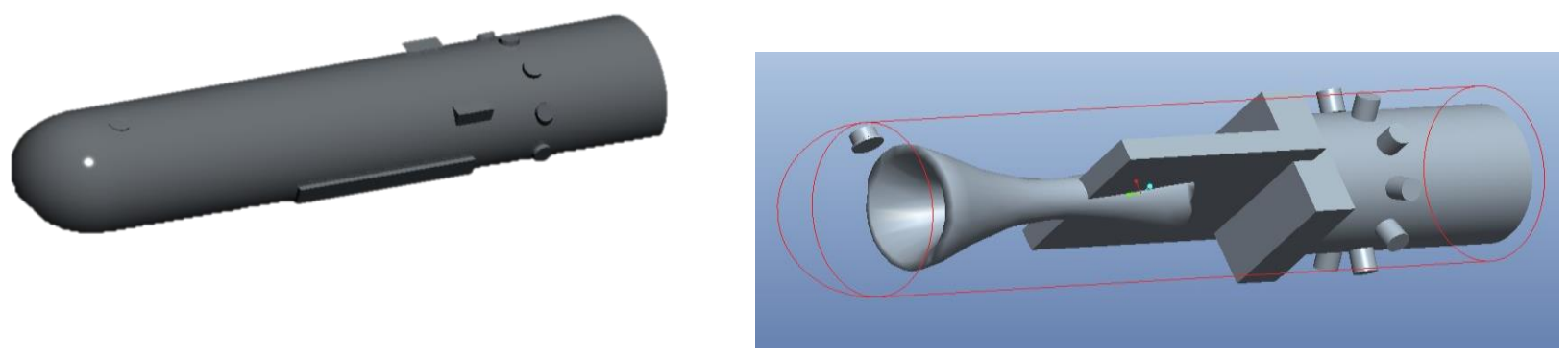

Figure 17: 0.75m Diameter Fairing CAD Model of ECS / Spacecraft
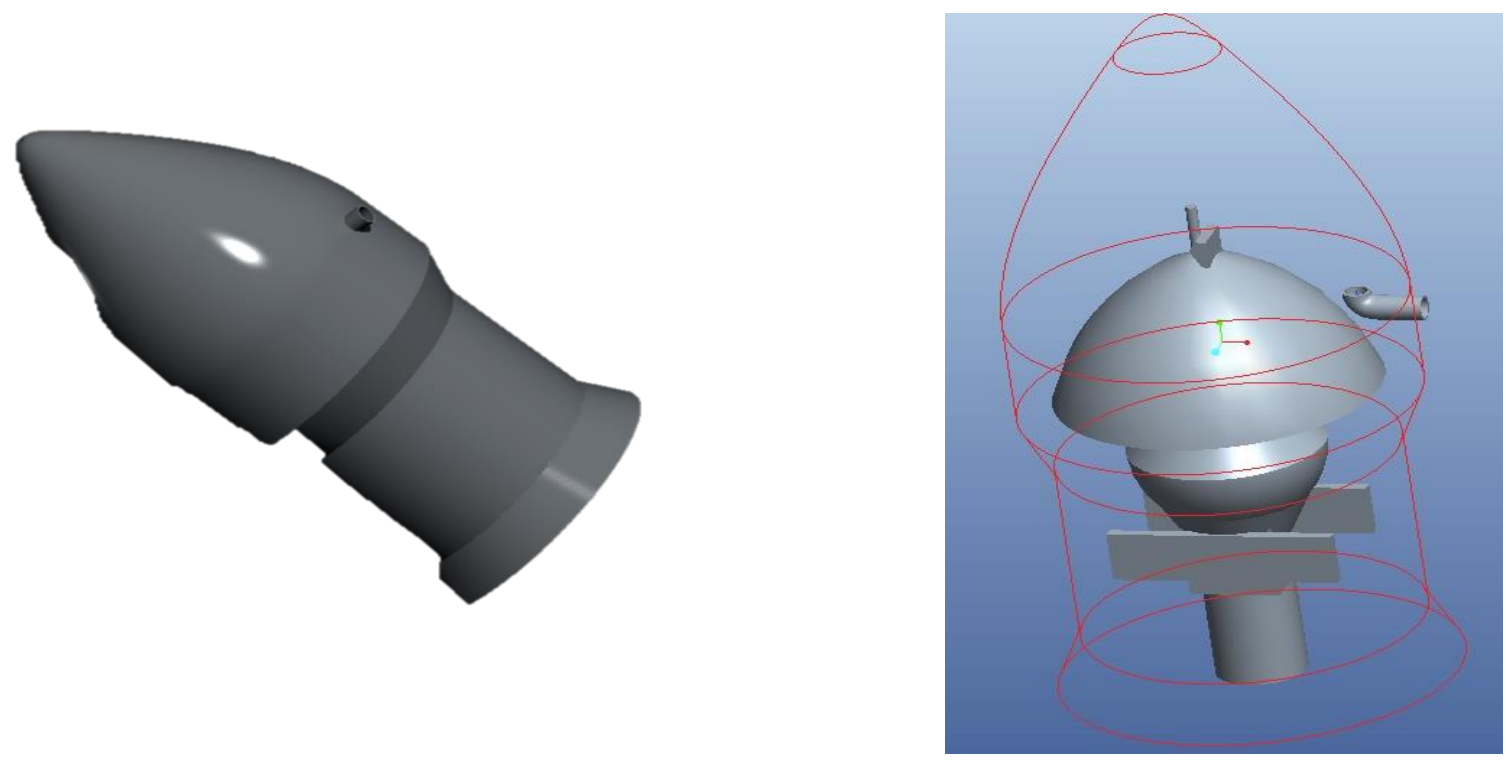

Figure 18: 3.5m Diameter Fairing CAD Model of ECS / Spacecraft 

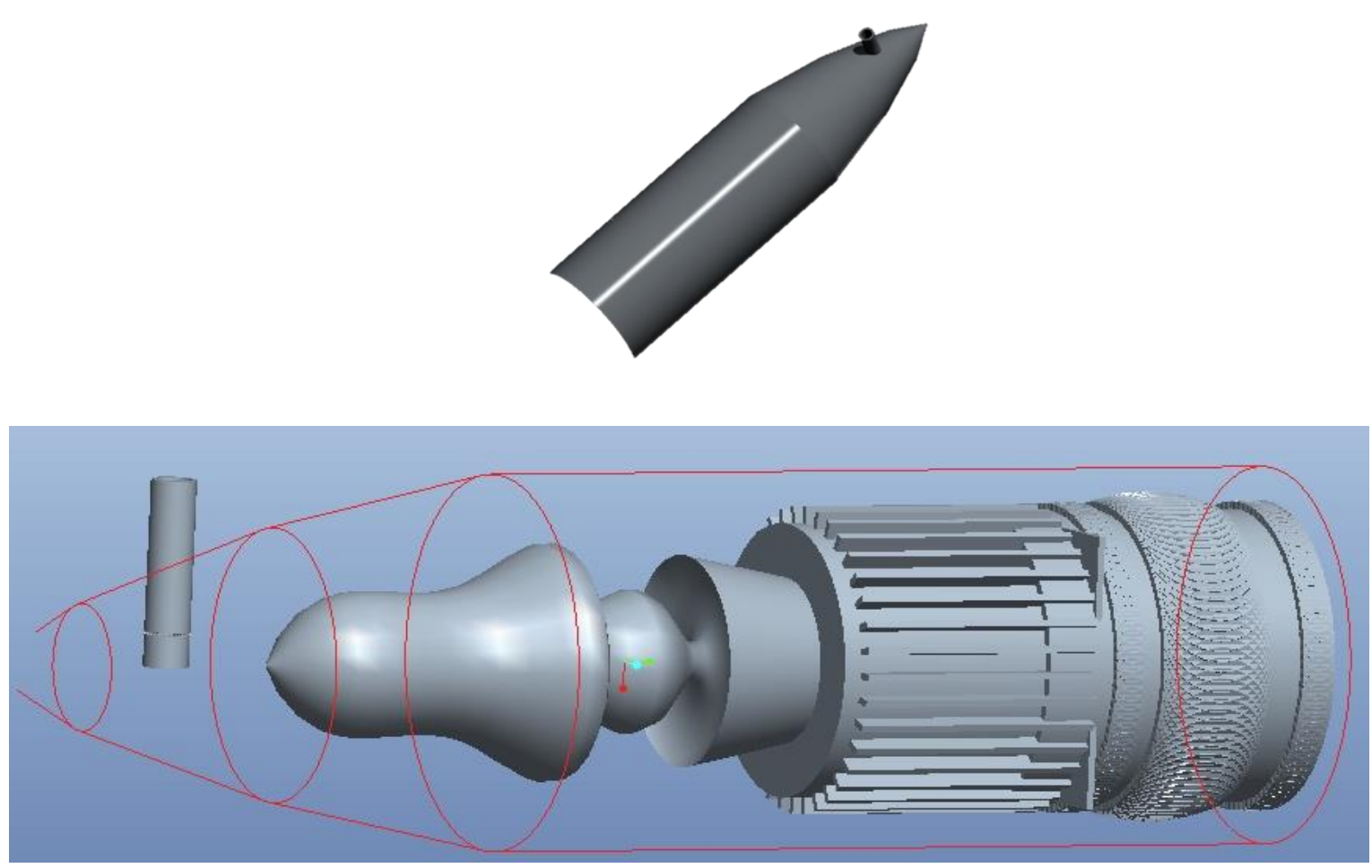

Figure 19: 5.5m Diameter Fairing CAD Model of ECS / Spacecraft

OPENFOAM was used as the primary solver. FLUENT and STARCCM+ were only used to access the uncertainty of the solver. OPENFOAM is more versatile for this research problem due to the open source code and no licensing issues. OPENFOAM additionally has the capability through snappy hex-mesh to import the CAD as an .STL files and mesh the geometry. Each of the CAD models shown above were created in Pro/ENGINEER were translated into an .STL file and meshed using snappy hex-mesh. The 0.75 meter geometry is shown in Figure 20, 3.5 meter geometry in Figure 21, and the $5.5 \mathrm{~m}$ geometry shown in Figure 22. 

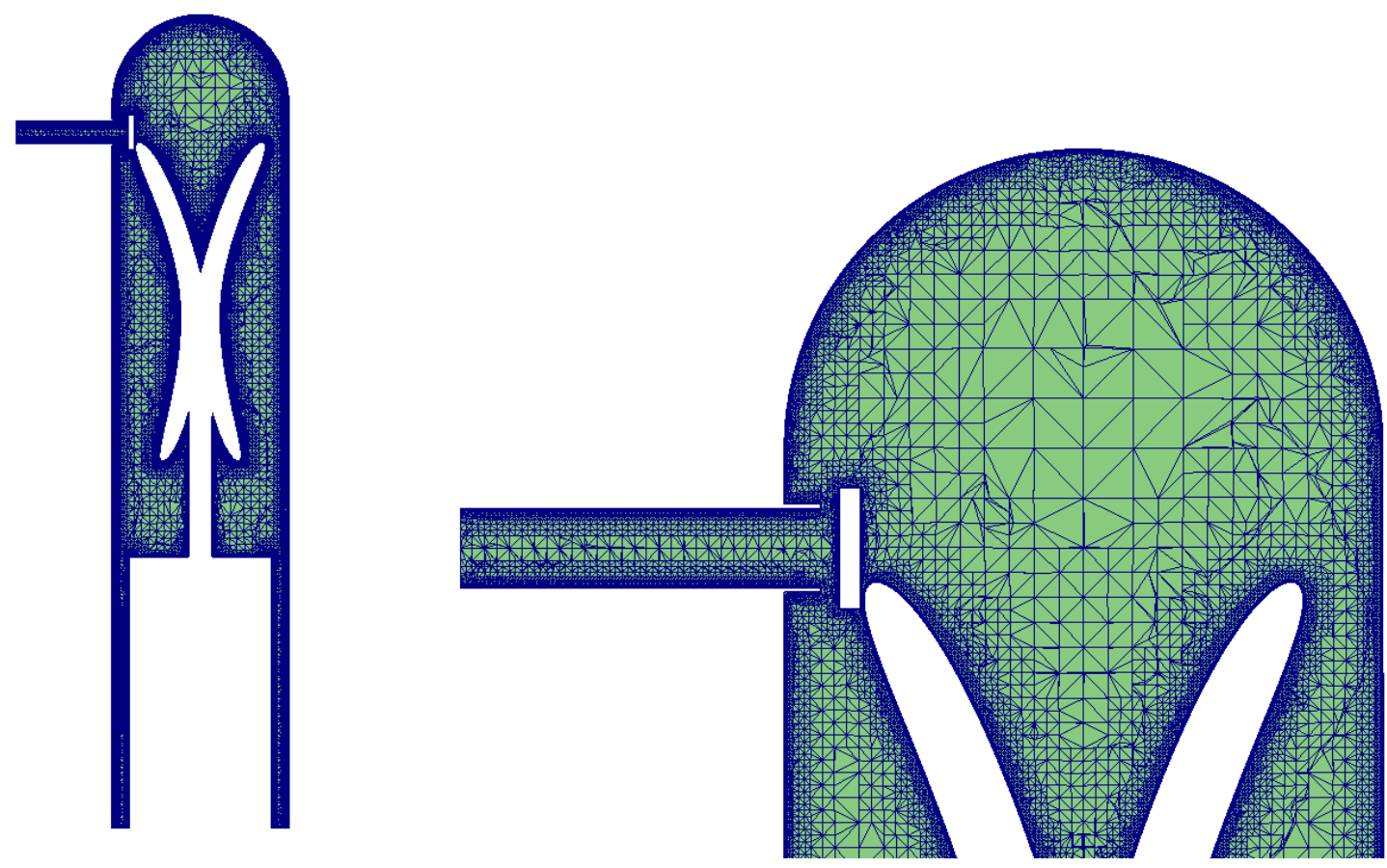

Figure 20: OpenFOAM SnappyHex Mesh Modeling CAD Model of ECS / Spacecraft $-0.75 \mathrm{~m}$ Configuration (6762865 number of cells)
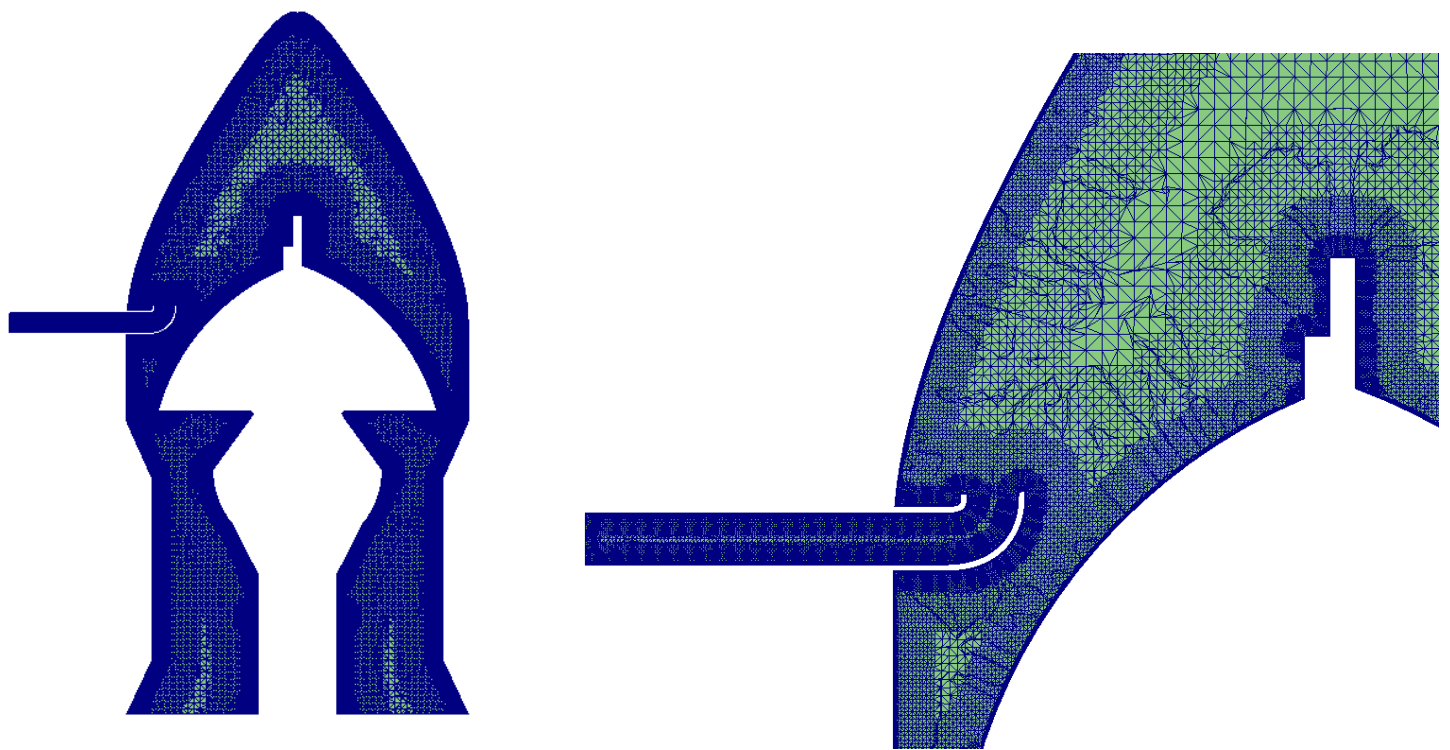

Figure 21: OpenFOAM SnappyHex Mesh Modeling CAD Model of ECS / Spacecraft - 3.5m Configuration (8594480 number of cells) 

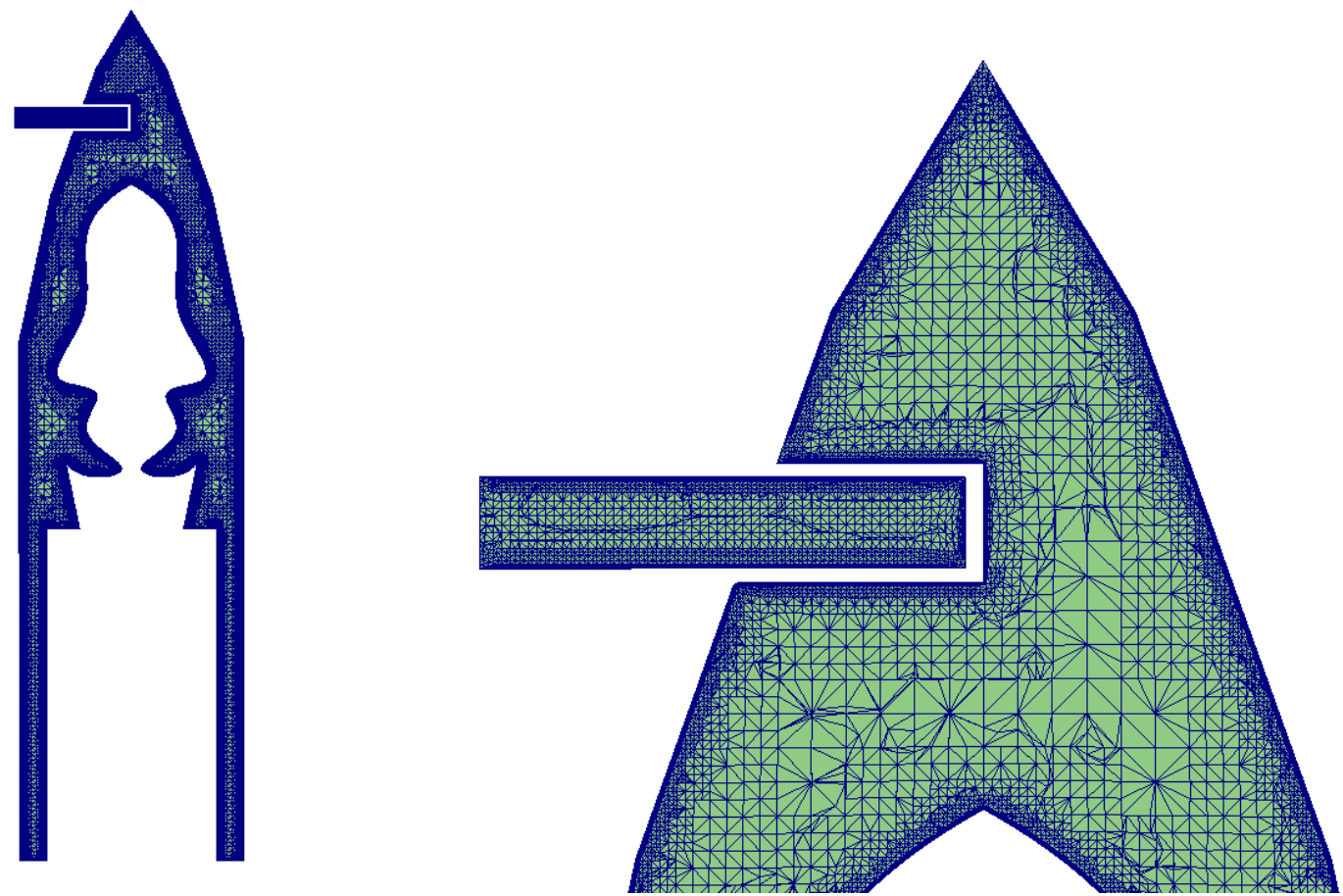

Figure 22: OpenFOAM SnappyHex Mesh Modeling CAD Model of ECS / Spacecraft - 5.5m Configuration (6980673number of cells)

The results from the CFD modeling using OPENFOAM's simpleFoam are displayed using ParaFoam. The velocity contours are shown for each configuration in Figure 23, Figure 24, and Figure 25, respectively. 


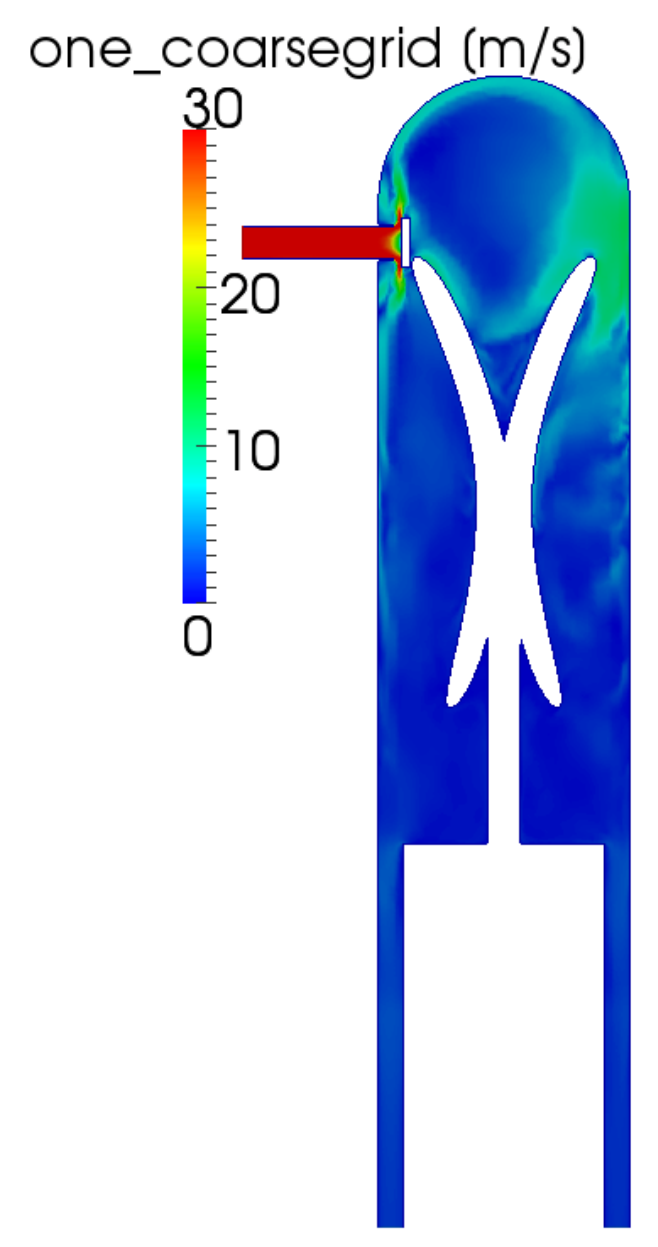

Figure 23: OpenFOAM SimpleFOAM Velocity Contours of ECS / Spacecraft - 0.75m Configuration (6762865 number of cells) 


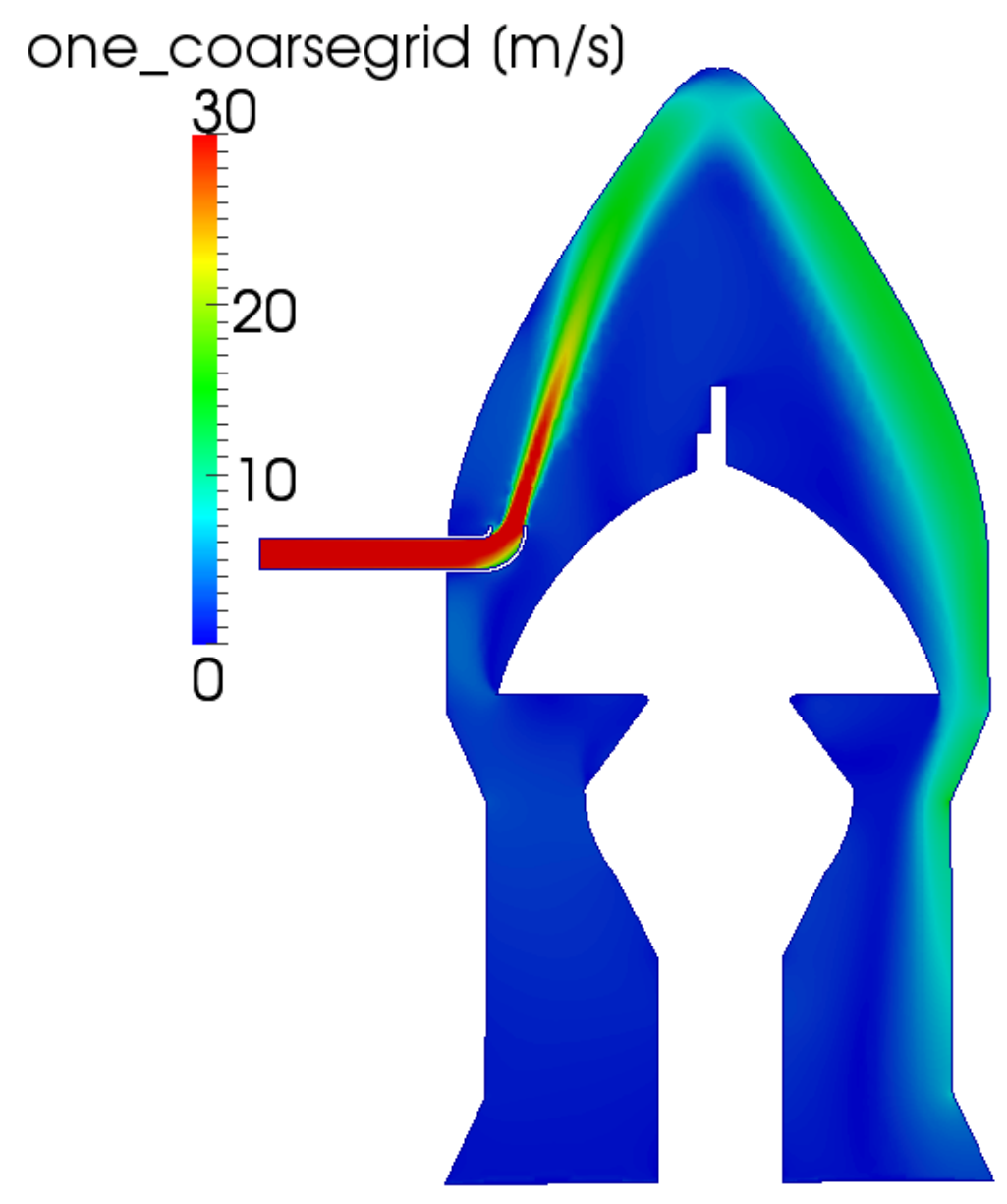

Figure 24: OpenFOAM SimpleFOAM Velocity Contours of ECS / Spacecraft - 3.5m Configuration (6980673number of cells) 


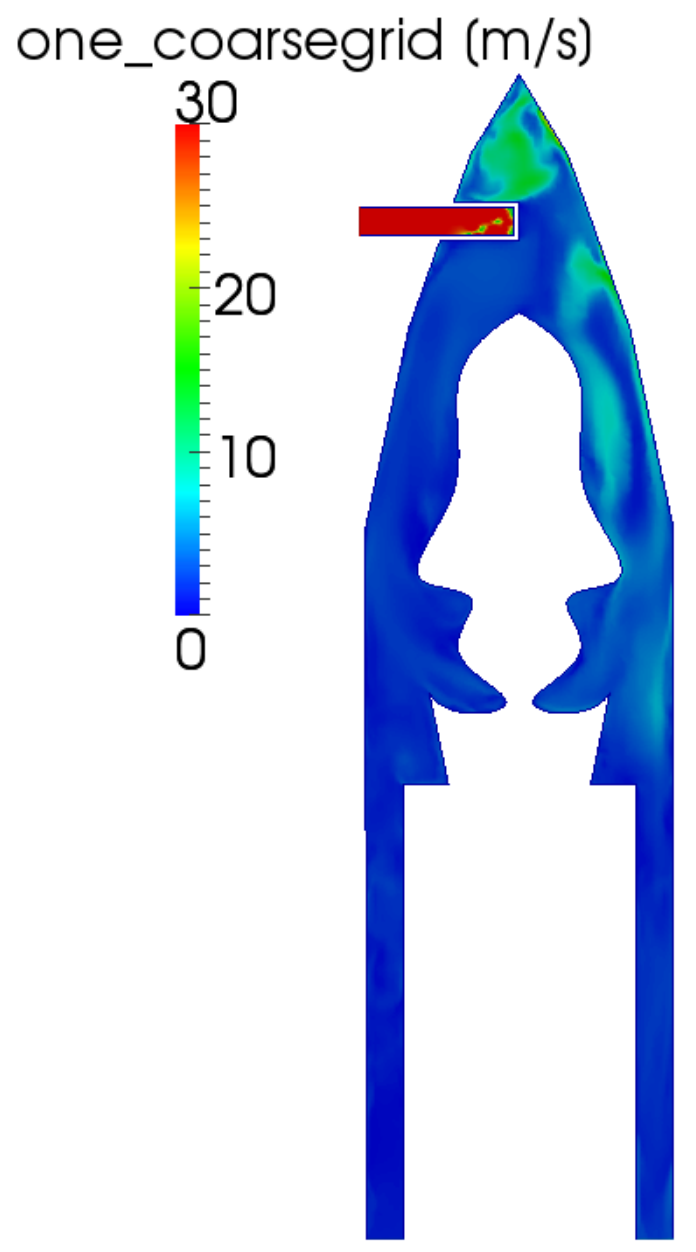

Figure 25: OpenFOAM SimpleFOAM Velocity Contours of ECS / Spacecraft $-5.5 \mathrm{~m}$ Configuration (6980673number of cells) 


\section{CHAPTER FIVE: COMPUTATIONAL FLUID DYNAMICS UNCERTAINTY ANALYSIS}

The uncertainty of using CFD to analyze the ECS system for airflow velocities around spacecraft is unknown and not documented. The objective of this section is to apply the methodology laid out in section 2.3. There are several items needing to be addressed in the section. First, the interpolation scheme used for the backward step is unacceptable. The interpolation scheme is introducing errors may make using the monotonic (Richardson's extrapolation method) un-realistic. A better interpolation scheme between the three grids will be sought. Second, using the monotonic numerical results and extrapolating a solution for the entire computational domain will need to be assessed for feasibility. It may prove using the grid as a separate oscillatory input parameter will suffice. To complete this objective a Comprehensive Approach to Verification and Validation of CFD Simulations - ASME Journal of Fluids Methodology outlined in previous section 2 will be used as a starting point and any inconsistencies or issues will be analyzed and solutions recommended. Again summarizing the method, three separate grids (rough, medium, fine) along with the uncertainty of all input parameters will be used to evaluate the uncertainty in the CFD velocity prediction. The velocity at every point in each of the three solutions will be compared to one another.

Before proceeding directly into the spacecraft/ECS system, two simpler problems are analyzed using the proposed methodology (laminar flow between parallel stationary plates and heat transfer over a flat plate). 


\subsection{Interpolation Scheme Needed for CFD Uncertainty Analysis}

The author published the work in this section as AIAA-2014-1433 [32]. CFD is used in many forums to approximate flow solutions of the Navier-Stokes equations. The Navier-Stokes equations are second order, non-homogenous, non-linear partial differential equations. Several papers have been published on the use of progressive grid refinement to estimate the errors in a CFD Simulation [8], [5], [33], [9], [7], [6], [10], [11], [12], [14], [19]. The procedure is to compare the differences in the solutions between at least three different grids. The computational domain discretization (grid) is significantly different in terms of the number of cells. This requires an interpolation between the grids and solutions to approximate the error. This interpolation will induce errors and the extrapolated uncertainty estimates become unreasonable and inaccurate [19]. The commercially available code ANSYS FLUENT includes mesh-to-mesh interpolation functionality [34]. This method performs a zeroth-order interpolation (nearest neighbor) for interpolating the solution data from one mesh to another [34]. This functionality is used to initialize data from one mesh on to another mesh for the purpose of an initial condition only. Using this method to approximate errors is inappropriate. OPENFOAM is an open source solver, which includes a similar functionality to the FLUENT mesh-to-mesh interpolation using a "mapfields" function [35]. The mapfields function is also designed as an initial guess to be used when iterating a solution. Using the FLUENT interpolation file or OpenFOAM mapfields to estimate errors in a grid convergence study will produce unrealistic results. The purpose of this section is to compare several other interpolation schemes which may be used for post-processing different solutions on different grids for the purpose of uncertainty estimation. 
Matlab is a high-level language used for numerical computations and includes several interpolation functions for one-dimensional data, uniformly spaced, gridded data in two and three dimensions, and scattered data interpolation [36]. CFD data comes in various forms, 1D, 2D, 3D, uniform, and non-uniform data. Matlab offers interp1, interp2, and interp3 for the corresponding dimensions. Interp1,2,3 includes the following schemes as shown in Table 3.

Table 3: Interpolation Schemes Available in Matlab

\begin{tabular}{|c|c|c|c|}
\hline & \multicolumn{3}{|c|}{ Matlab Function } \\
\hline & interp1 & interp2 & interp3 \\
\hline \multicolumn{4}{|l|}{ Interpolation Method } \\
\hline 'nearest' - Nearest neighbor interpolation & $\mathrm{X}$ & $\mathrm{X}$ & $\mathrm{X}$ \\
\hline 'linear' - Linear interpolation (default) & $\mathrm{X}$ & $\mathrm{X}$ & $\mathrm{X}$ \\
\hline 'spline' - Cubic spline interpolation & $\mathrm{X}$ & $\mathrm{X}$ & $\mathrm{X}$ \\
\hline 'pchip' - Piecewise cubic Hermite interpolation & $\mathrm{X}$ & & \\
\hline 'cubic' & $\mathrm{X}$ & $\mathrm{X}$ (uniformly-spaced only) & $\mathrm{X}$ (uniformly-spaced only) \\
\hline 'v5cubic' - cubic interpolation used in Matlab 5 & $X$ & & \\
\hline
\end{tabular}

A generic scheme is sought that would be available for $1 \mathrm{D}, 2 \mathrm{D}, 3 \mathrm{D}$, uniform, and non-uniform grids. The three schemes available are "nearest", "linear", and "spline". The nearest is the same scheme available in the current CFD codes for mesh-to-mesh interpolation and would not provide additional benefit. The linear scheme and spline however could provide a better interpolation method for estimating numerical uncertainty in grid refinement studies.

Flow between parallel plates has an exact solution and provides a good example of the interpolation errors induced by using the "nearest" scheme and will provide a metric for comparing the errors in the "linear" and "spline" to an exact solution. 
Fully developed laminar flow between stationary, parallel plates is an exact solution to the Navier-Stokes Equations as derived in "Introduction to Fluid Mechanics" [37]. The width of the channel is (a).

$$
u=\frac{a^{2}}{2 \mu}\left(\frac{\partial P}{\partial x}\right)\left[\left(\frac{y}{a}\right)^{2}-\left(\frac{y}{a}\right)\right]
$$

A CFD model of this problem was created in FLUENT. The fluid is air. Table 4 outlines the parameters used.

Table 4: Parameters for Exact Solution

\begin{tabular}{|c|c|}
\hline $\mathrm{a}(\mathrm{m})$ & 0.1 \\
\hline $\mathrm{rho}(\mathrm{kg} / \mathrm{m} 3)$ & 1.225 \\
\hline $\mathrm{mu}(\mathrm{Ns} / \mathrm{m} 2)$ & 0.00001789 \\
\hline $\mathrm{dp} / \mathrm{dx}(\mathrm{N} / \mathrm{m} 3)$ & -0.0004 \\
\hline
\end{tabular}

The exact solution is shown in Figure 26.

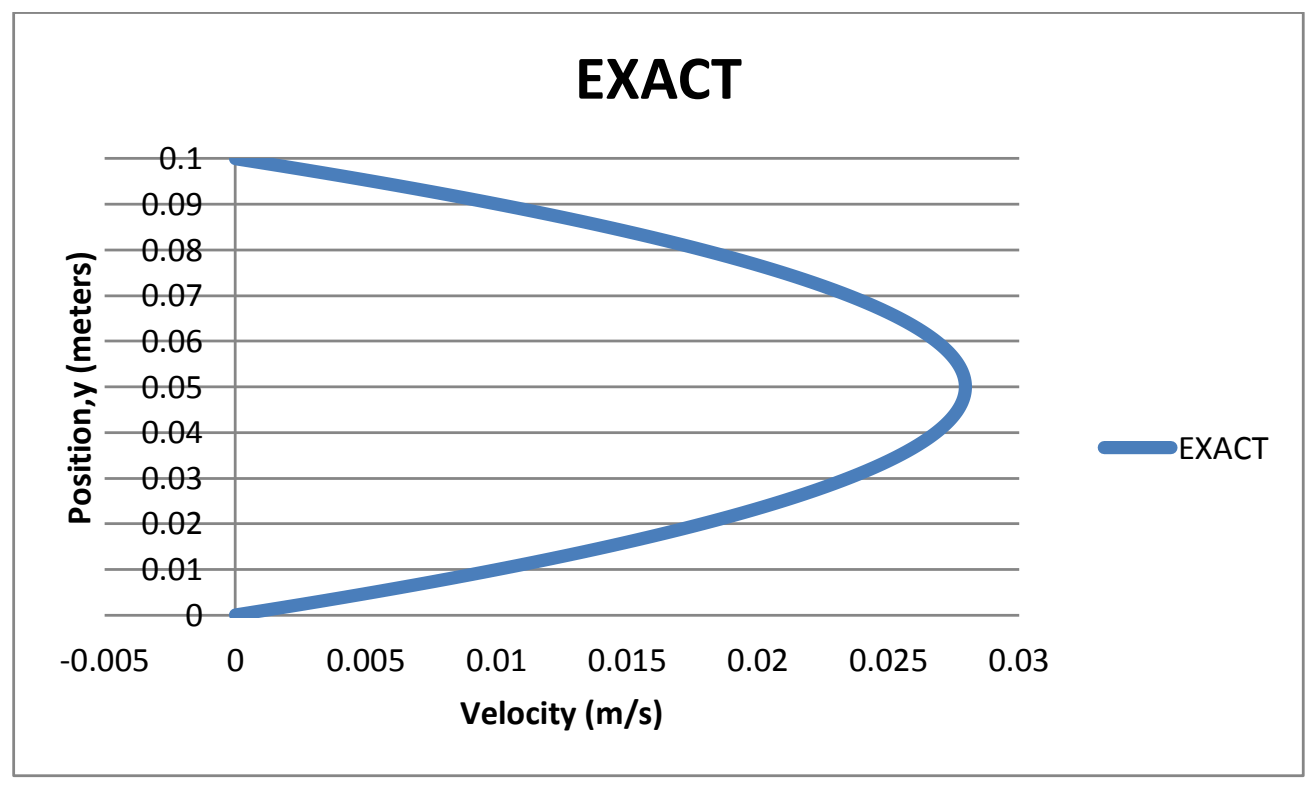

Figure 26: Exact Solution 
A CFD model was created for half of the domain. Flow between parallel plates has symmetry about the centerline. The inlet boundary condition used was the average velocity as shown in equation 16 and the domain was made long enough to be considered fully developed.

$$
\bar{V}=-\frac{1}{12 \mu}\left(\frac{\partial P}{\partial x}\right) a^{2}
$$

Three grids can be used to extrapolate an error. Three separate CFD models were created (coarse, medium, and fine). The coarse medium and fine grids have the following number of cells, $7140,14186,24780$, respectively. The three solutions plotted for flow between parallel plates and compared to the exact solution are shown in Figure 27.

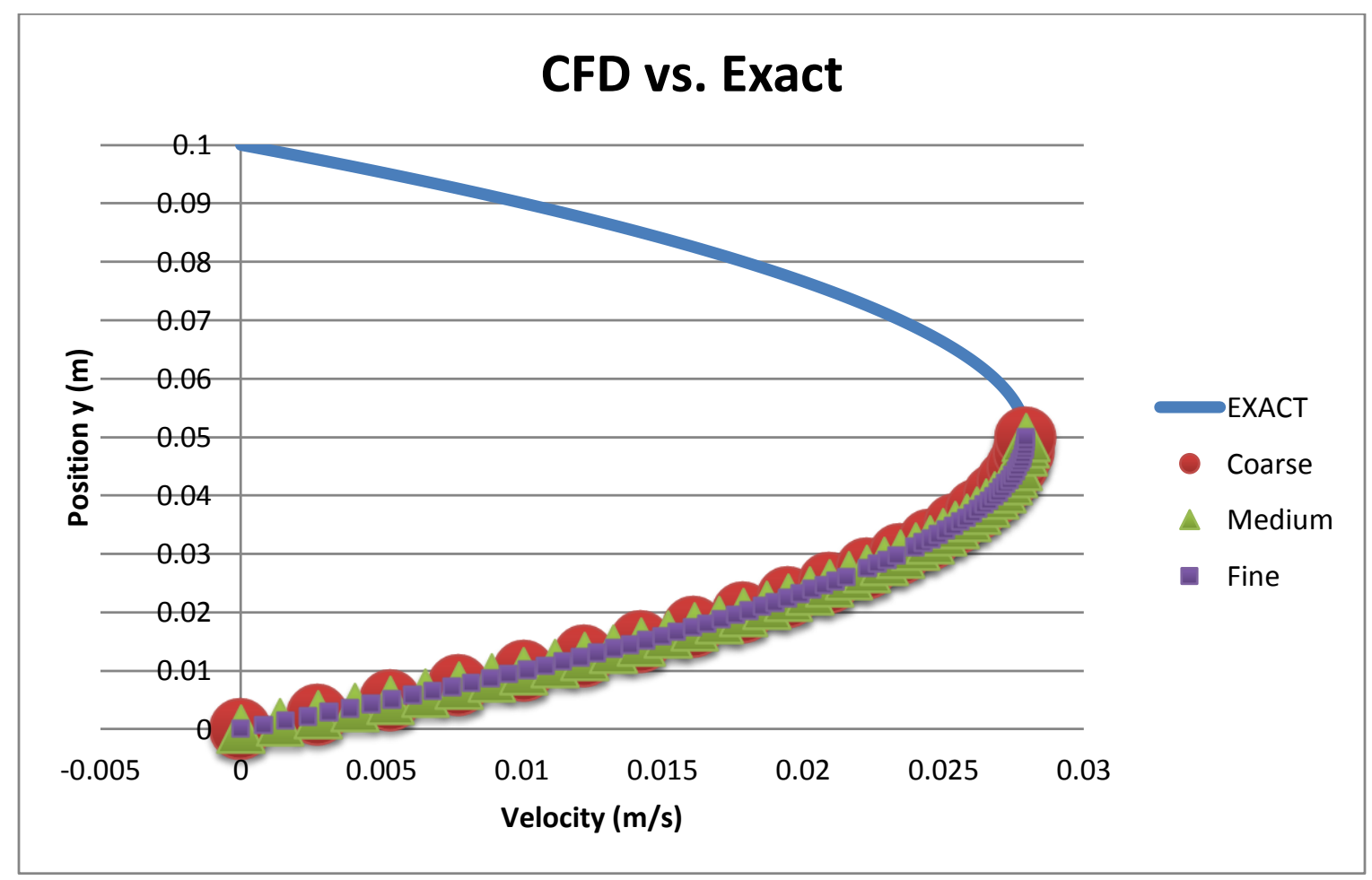

Figure 27: CFD Results (coarse, medium, fine) vs. Exact Solution 
The interpolation methods outlined "nearest", "linear", and "cubic" were investigated by interpolating the results from the fine grid and medium grid onto the coarse grid. The coarse grid was chosen because ideally it should be good enough to approximate the solution and all recommendations for normal grid refinement already followed. The medium and fine grids are used only for the error approximation.

FLUENT's mesh-to-mesh interpolation functionality was used and the results are shown in Figure 28. From a plot of the entire computational domain, the reader would not be able to see the variation, so the plot was zoomed in to show the errors being induced by using the "nearest" interpolation.

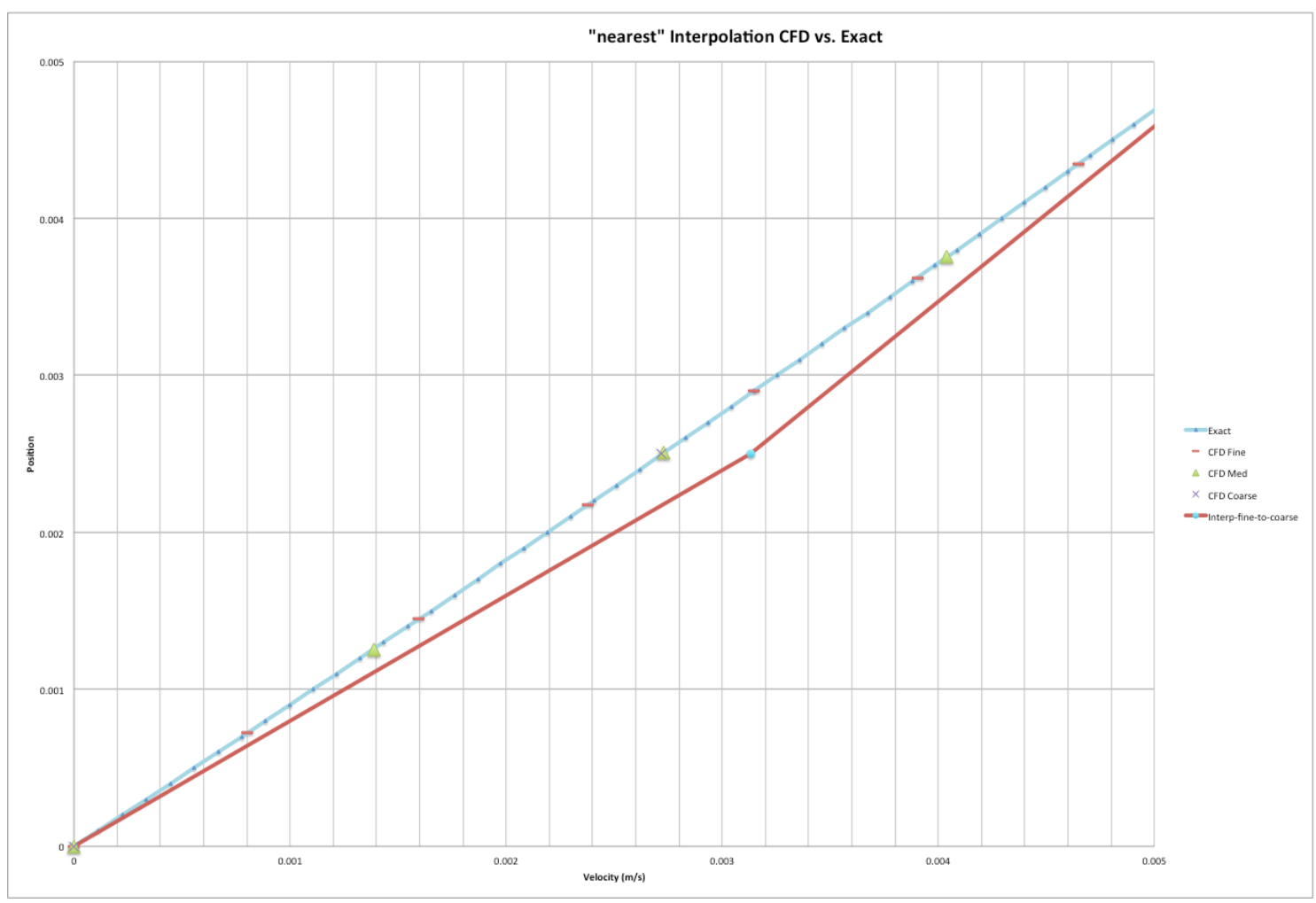

Figure 28: "nearest" interpolation of CFD Results (coarse, medium, fine) vs. Exact Solution 
The error induced by using the "nearest" interpolation scheme was as high as 15 percent of the exact value. Also, this high error was in the critical region closest to the wall.

To compare the linear interpolation scheme Matlab was used.

$$
\text { yfi =interp1(fine(:,2),fine(:,1),coarse(:,2),'linear') }
$$

The percent difference was greatly reduced to 0.08 percent of the exact solution and plotting the results does not visually show a difference as shown in Figure 29.

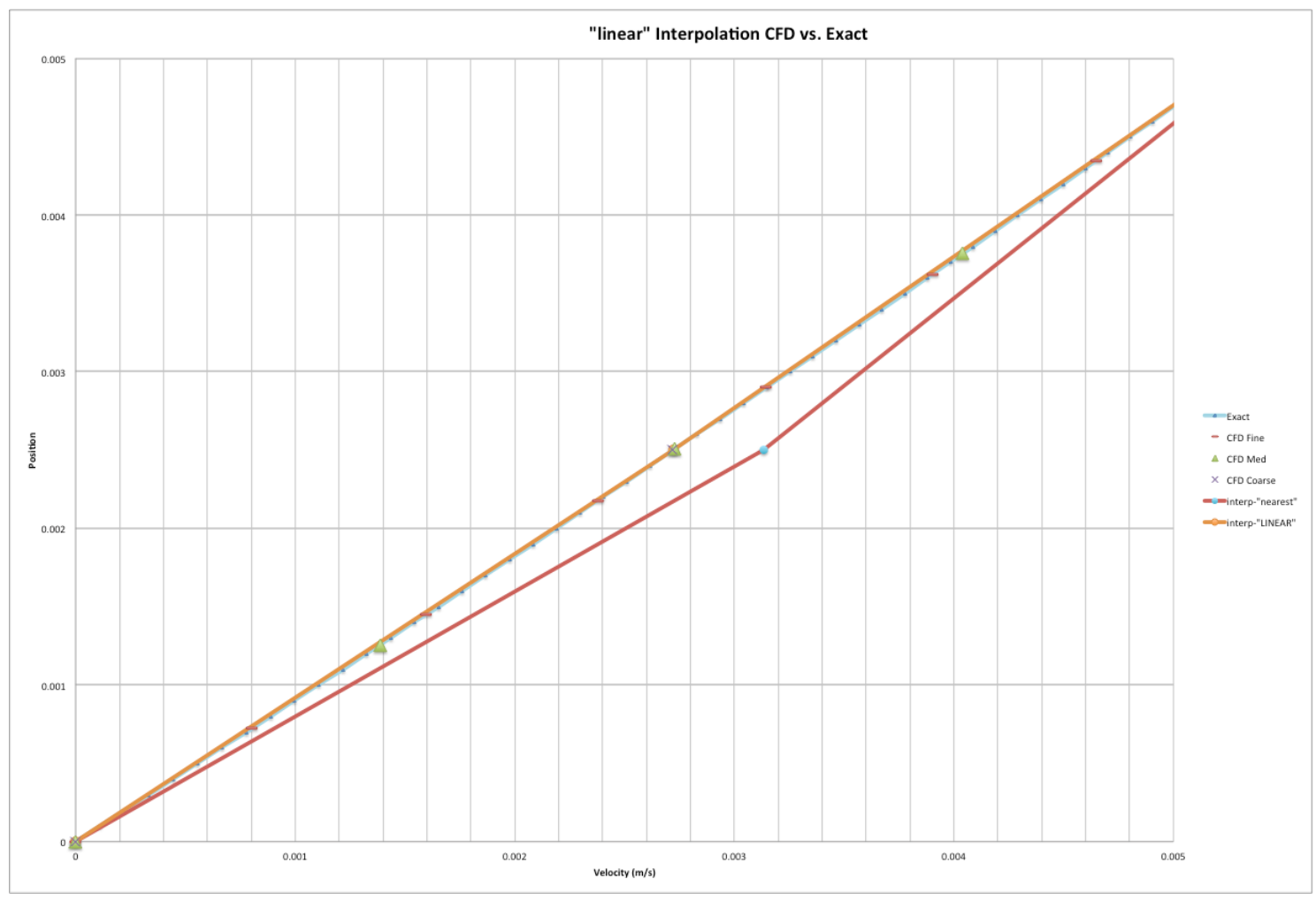

Figure 29: "linear" interpolation of CFD Results (coarse, medium, fine) vs. Exact Solution 
To compare the cubic interpolation scheme, Matlab was again used as follows.

$$
\text { yfi = interp1(fine(:,2),fine(:,1),coarse(:,2),'cubic') }
$$

The percent difference was even further reduced to 0.07 percent of the exact solution and plotting the results does not visually show a difference as shown in Figure 30.

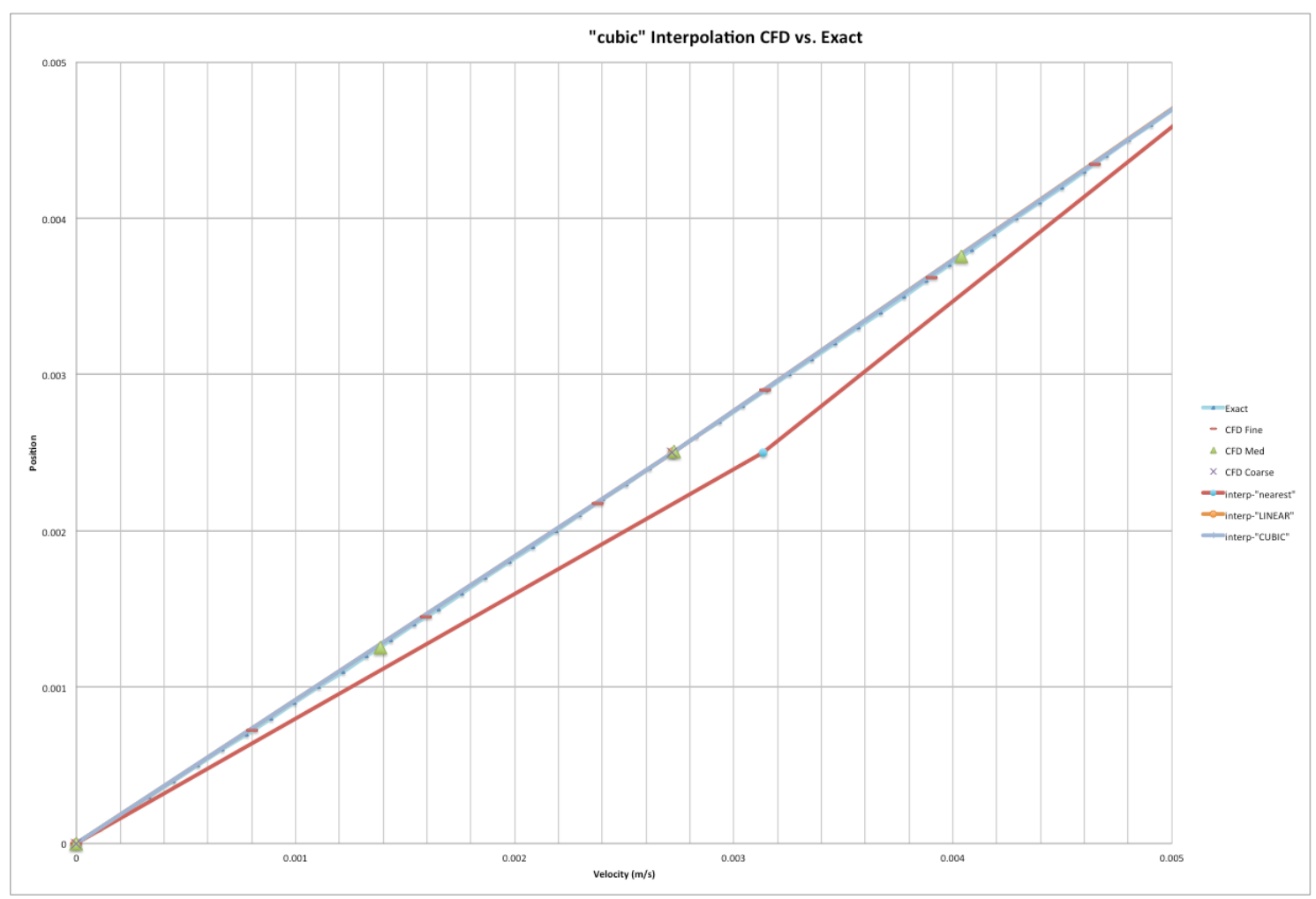

Figure 30: "cubic" interpolation of CFD Results (coarse, medium, fine) vs. Exact Solution 
The method was extended to 2D and 3D. Matlab uses a meshgrid function, which is needed before using the interp2 or interp3 functions. Using the meshgrid function transforms the domain specified by vectors into arrays. Performing this option for CFD meshes in the 4 million to 8 million cells yields a "maximum variable size allowed by the program is exceeded". Using the interp2 or interp3 did not provide a feasible way to perform the interpolation.

Matlab contains “griddata” function, which includes the following methods ('nearest', 'linear', 'natural', 'cubic', and 'v4'). The only options available in both 2D and 3D are 'nearest', 'linear', and 'natural'. Again, a general method is sought that works in 1D, 2D, and 3D. The only options available in interp1 and griddata are 'nearest' and 'linear'. The linear method is considered only from this point forward.

A CFD model was created for a 3D internal flow problem and three grids considered. The 'linear' option was used to interpolate between the fine and coarse grids. The interpolation does induce some error between the meshes; however, the error is very similar to the variation between the grids. Figure 31 shows the velocity contour plots between the fine grid and the coarse grid. The contour plots are visually identical.

A line plot was constructed comparing the three grids and the interpolated solutions as shown in Figure 32. The line is extracted from the contour plot as shown with the pink line in Figure 33. 


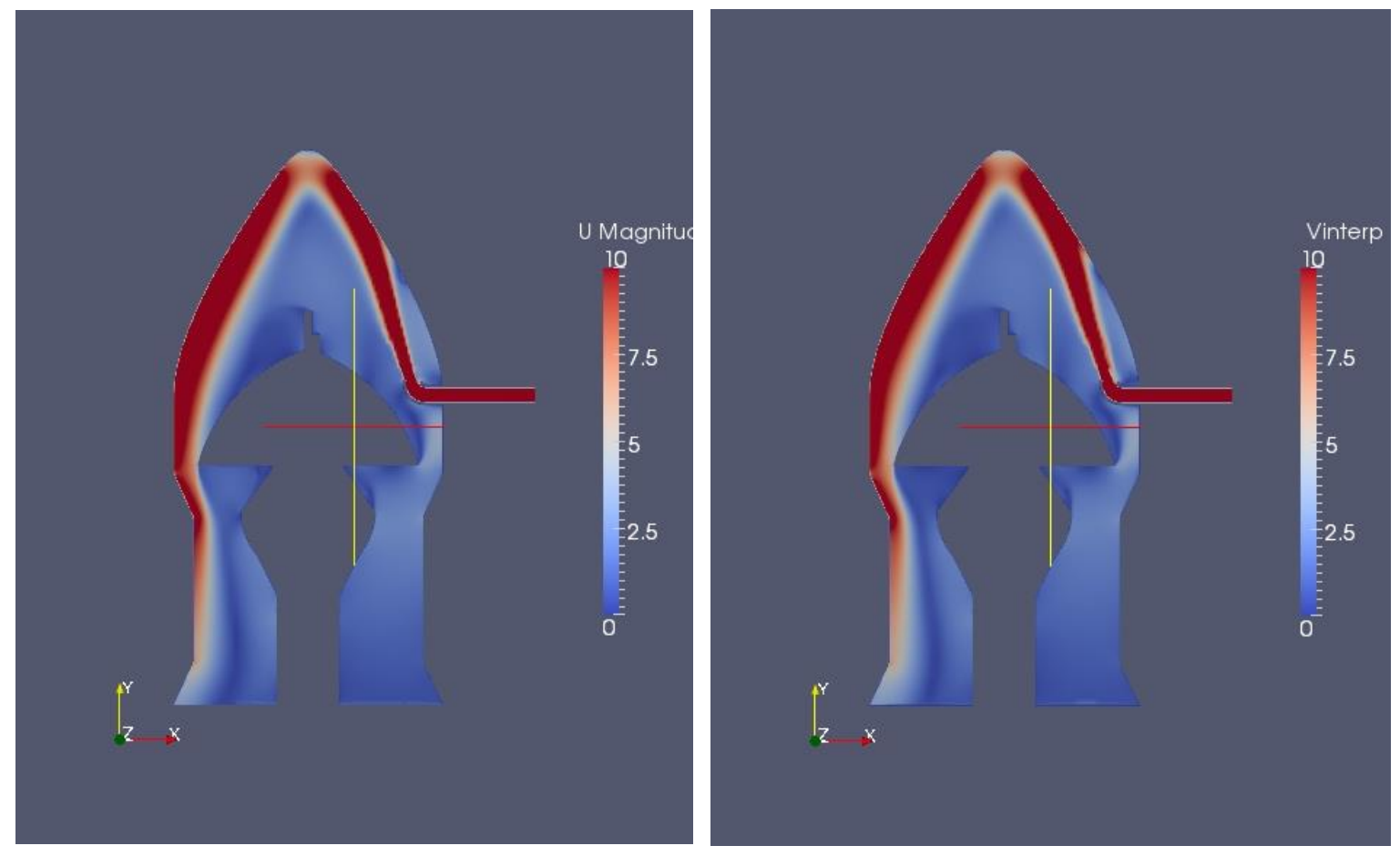

Figure 31: 3D Linear Interpolation Contour Plot vs. Fine Solution
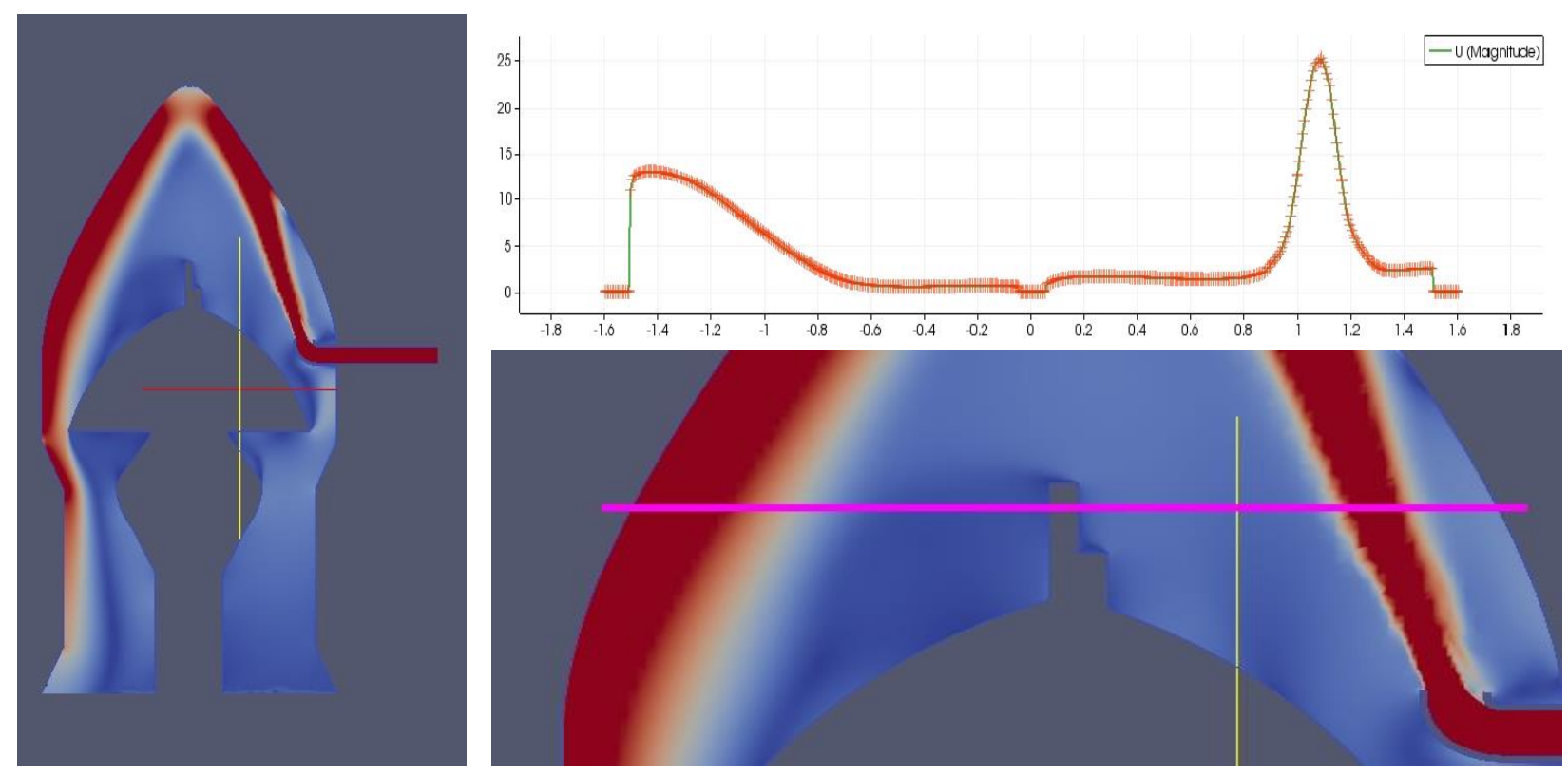

Figure 32: 3D Linear Interpolation Line Plot 


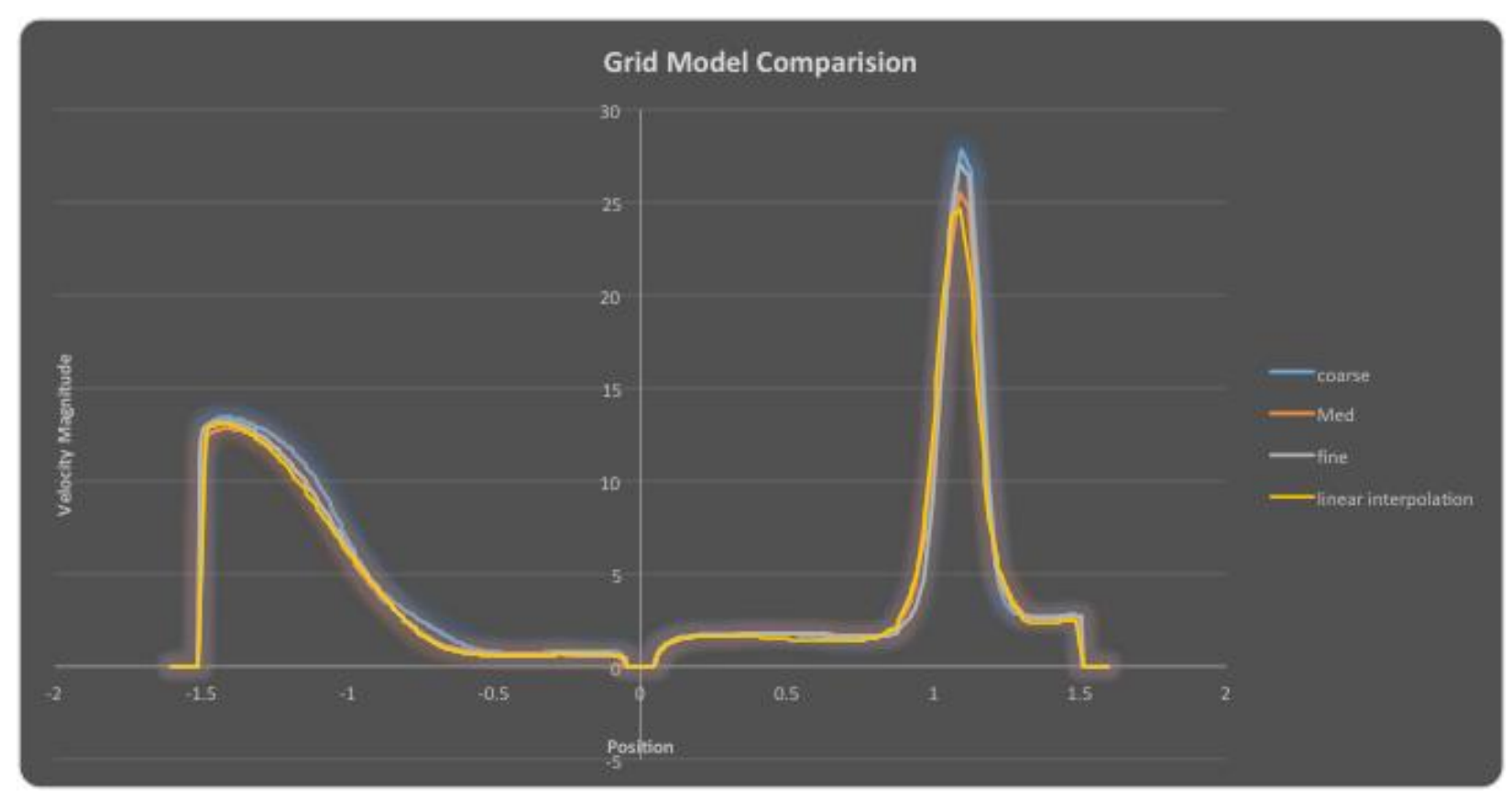

Figure 33: 3D Linear Interpolation Line Plot vs. Fine, Medium, and Coarse Solutions

By comparing the interpolation schemes in one, two, and three dimensions and investigating the options readily available in Matlab, it is recommended the "linear" option be used when comparing the error or uncertainty due to the grid. The matlab code used for this interpolation is provided in Appendix A: InterpOPENFOAM.m.

There is another issue with interpolation. Which direction to use? 


\section{Interpolate Coarse and Medium Mesh -> Fine}
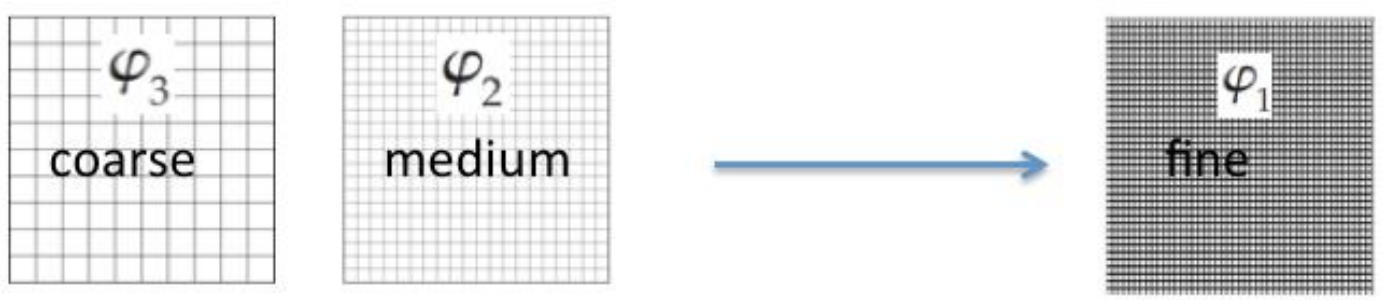

\section{Interpolate Medium and Fine Mesh -> Coarse}
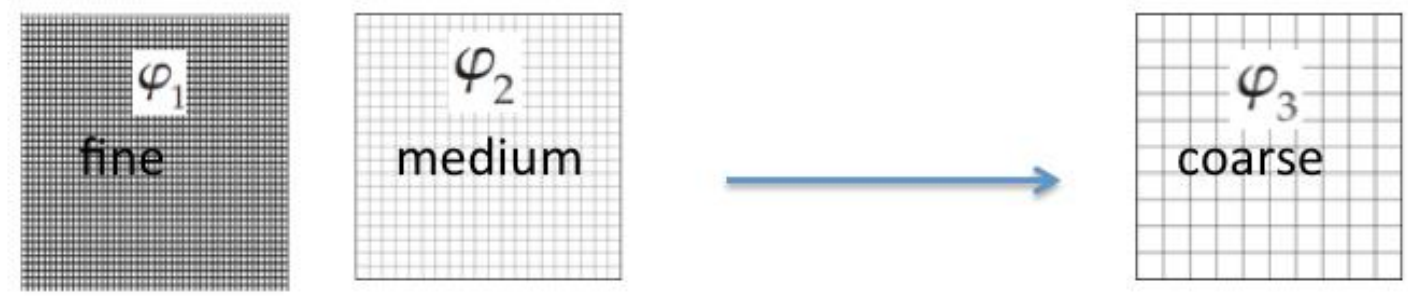

Figure 34: Interpolation Direction

The paper provided by Roach and Knupp suggests, "There is no value in producing two grids with the fine and coarse spacings. Only the fine grid need to be generated, and the course grid formed by using every other point" [38]. The paper uses the fine grid extrapolated solution. This is equivalent to interpolating via option1, all grids get interpolated onto the fine grid and the extrapolated solution is about the fine grid. The interpolation direction was investigated by further comparing the schemes and Richardson's extrapolation to the exact solution of parallel flow between parallel plates via the 5 step procedure. The results are shown in Figure 35, Figure 36 and Table 5, Table 6, Table 7. 

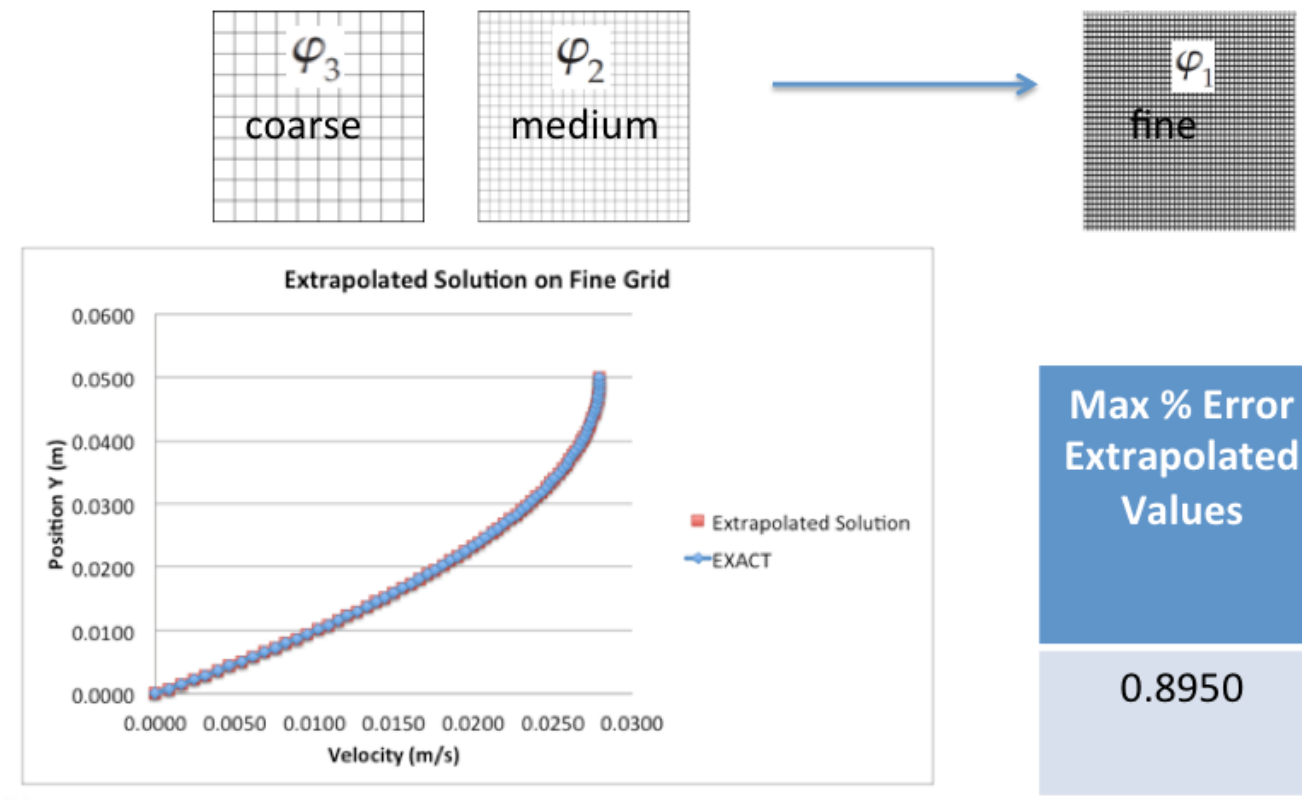

\begin{tabular}{|c|c|}
\hline $\begin{array}{c}\text { Max \% Error } \\
\text { Extrapolated } \\
\text { Values }\end{array}$ & $\begin{array}{c}\text { Average \% } \\
\text { Error } \\
\text { Extrapolated } \\
\text { Values }\end{array}$ \\
\hline 0.8950 & 0.0596 \\
\hline
\end{tabular}

Figure 35: Interpolation Direction onto Fine Grid
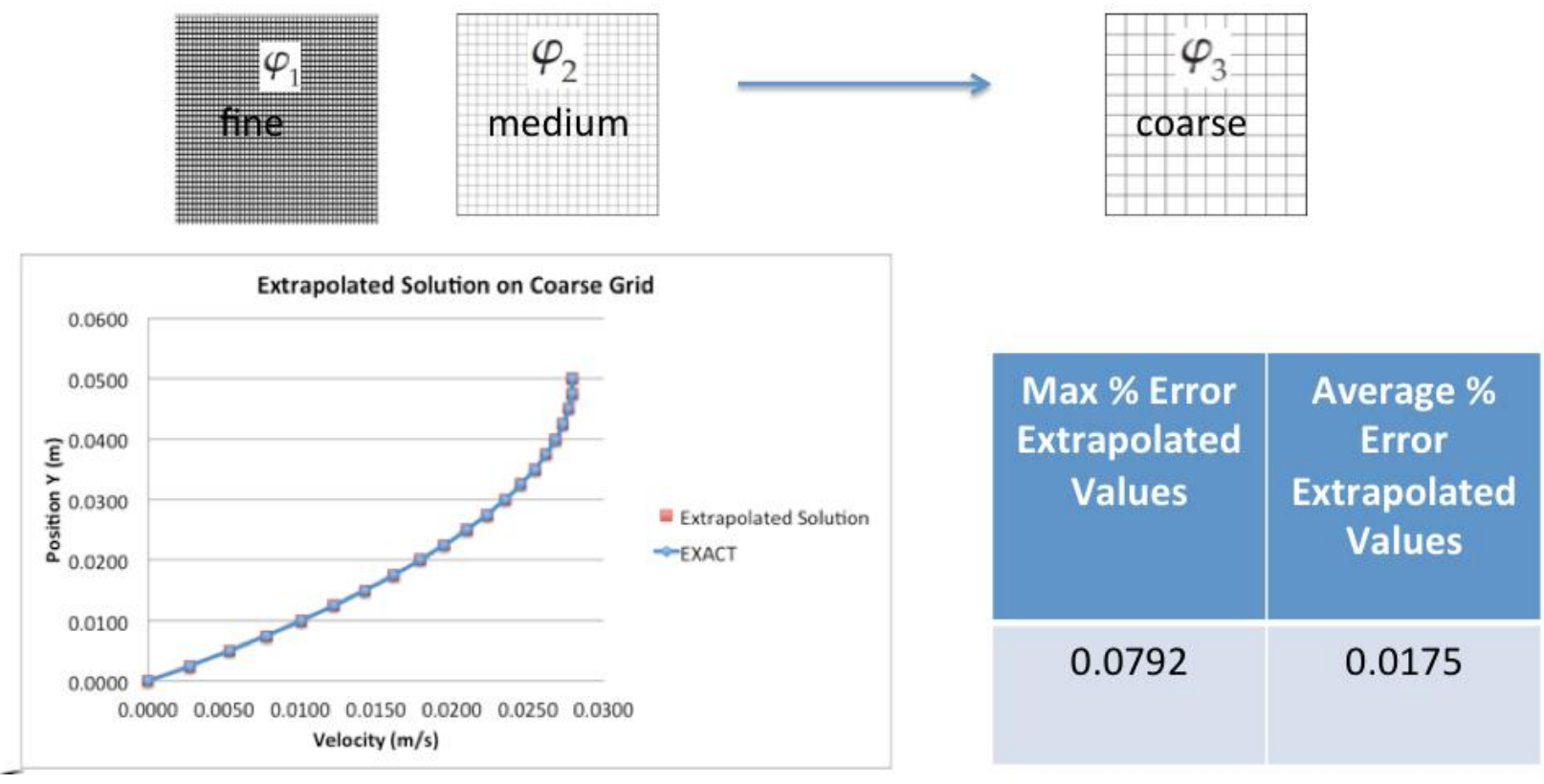

Figure 36: Interpolation Direction onto Coarse Grid 
Table 5: Results Interpolating to Fine Grid for Flow Between Parallel Plates

\begin{tabular}{|l|r|}
\hline \multicolumn{2}{|c|}{ Interpolation to Fine Grid 'Linear' } \\
\hline Average Observed Order, $p$ & 5.0441 \\
\hline Max \% Error of Extrapolated Values & 0.8950 \\
\hline Average \% Error of Extrapolated & 0.0596 \\
\hline \multicolumn{1}{|l|}{ Intues } & \\
\hline Interpolation to Fine Grid 'Cubic' & 5.4401 \\
\hline Average Observed Order, $p$ & 1.1612 \\
\hline Max \% Error of Extrapolated Values & \\
\hline Average \% Error of Extrapolated & \\
Values & \\
\hline
\end{tabular}


Table 6: Results Interpolating to Coarse Grid for Flow Between Parallel Plates

\begin{tabular}{|l|r|}
\hline \multicolumn{2}{|c|}{ Interpolation to Coarse Grid 'Linear' } \\
\hline Average Observed Order, $\mathrm{p}$ & 7.5200 \\
\hline Max \% Error of Extrapolated Values & 0.0792 \\
\hline Average \% Error of Extrapolated & \\
Values & 0.0175 \\
\hline \multicolumn{2}{|l|}{} \\
\hline Interpolation to Coarse Grid 'Cubic' \\
\hline Average Observed Order, p \\
\hline Max \% Error of Extrapolated Values \\
\hline $\begin{array}{l}\text { Average \% Error of Extrapolated } \\
\text { Values }\end{array}$ \\
\hline
\end{tabular}


Table 7: Comparison of Interpolation Results

\begin{tabular}{|c|c|c|}
\cline { 2 - 3 } \multicolumn{1}{c|}{ Grids } & $\begin{array}{c}\text { Max \% } \\
\text { Error }\end{array}$ & $\begin{array}{c}\text { Average \% } \\
\text { Error }\end{array}$ \\
\hline (Coarse vs Exact) & 0.1910 & 0.1265 \\
\hline (Medium vs Exact) & 0.0969 & 0.0367 \\
\hline (Fine vs Exact) & 0.0289 & 0.0121 \\
\hline
\end{tabular}

1. Linearly Interpolated Coarse, Medium to Fine

\begin{tabular}{|c|c|c|}
\hline (Interpolated Coarse vs Exact) & 1.9760 & 0.2528 \\
\hline (Interpolated Medium vs Exact) & 0.6322 & 0.0679 \\
\hline
\end{tabular}

2. Linearly Interpolated Medium, Fine to Coarse

\begin{tabular}{|c|c|c|}
\hline (Interpolated Medium vs Exact) & 0.0728 & 0.0362 \\
\hline (Interpolated Fine vs Exact) & 0.0787 & 0.0223 \\
\hline
\end{tabular}

Extrapolated

\begin{tabular}{|c|c|c|}
\hline $\begin{array}{c}\text { 1. Linear Interpolation Coarse and Medium to Fine } \\
\text { (Extrapolated vs Exact) }\end{array}$ & 0.8950 & 0.0596 \\
\hline $\begin{array}{c}\text { 2. Linear Interpolation Medium and Fine to Coarse } \\
\text { (Extrapolated vs Exact) }\end{array}$ & 0.0792 & 0.0175 \\
\hline
\end{tabular}

It can be shown by interpolating the fine and medium grids onto the coarse grid and performing Richardson's Extrapolation provides better results. The average error between the extrapolated values and exact solution is reduced by factor of 3.4 for the 'linear' method and factor of 14 for the 'cubic' method. However, there is an underlying advantage to interpolating to the finest grid because the analyst has more data available. Also, by interpolating from the fine and medium 
grids onto the coarse grid to perform Richardson's Extrapolation, a higher observed order of convergence is accomplished and a lower error in the extrapolated solution.

\subsection{Feasibility of Using Richardson's Extrapolation for Entire Computational Domain}

To use Richardson's extrapolation, the solution must be monotonically increasing or decreasing with grid refinement. The solution is in the asymptotic range. By plotting some data in the computational domain for three different grids, it can be shown that increasing the grid does not provide a monotonic solution at every point. Please see Figure 37. The two_medgrid would have to be between the one_coarsegrid and three_fine grid at every location to use Richardson's extrapolation. Using this method to estimate uncertainty for the entire computational domain is inappropriate due to the grid refinement not being monotonically increasing or decreasing at every location.

Another way to present this argument is to look at the $R_{k}$ values as described in equation 11 . For all $R_{k}$ values greater than 1 , the solution is divergent. For all $R_{k}$ values between 0 and 1 , the solution is monotonic and Richardson's extrapolation may be used. For all $R_{k}$ values less than 0 , oscillatory convergence is prevalent. Figure 38, Figure 39, and Figure 40 are plots of the $R_{k}$ values for each configuration being analyzed. For the plots all values in blue represent cells with oscillatory convergence. All values shown in red represent the cells with divergence. All other colors represent cells with monotonic convergence. Therefore, it is inappropriate to use Richardson's extrapolation or the ASME 5 step procedure for estimating uncertainties associated with the numeric's. 


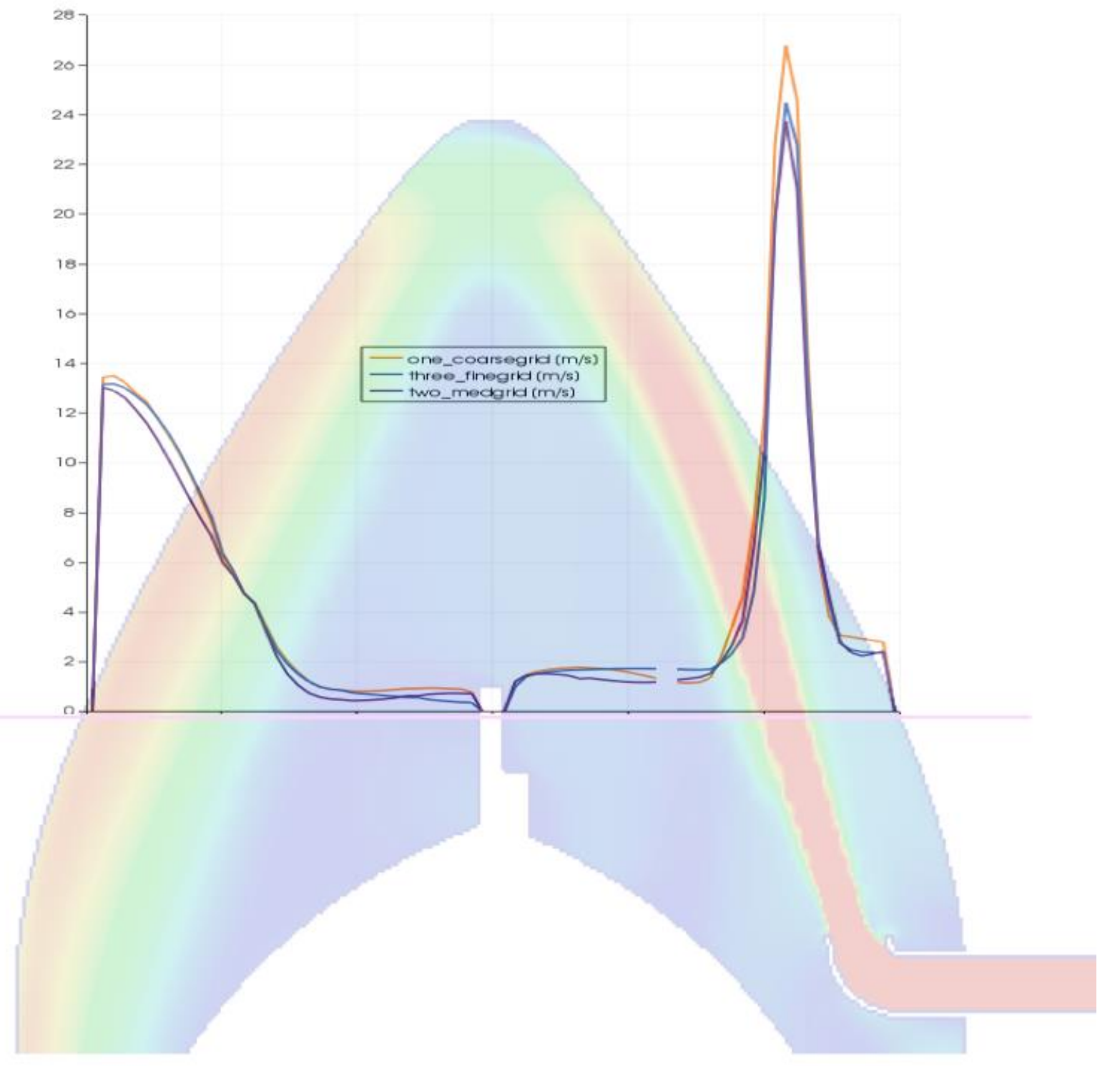

Figure 37: Fine, Medium, and Coarse Solutions 


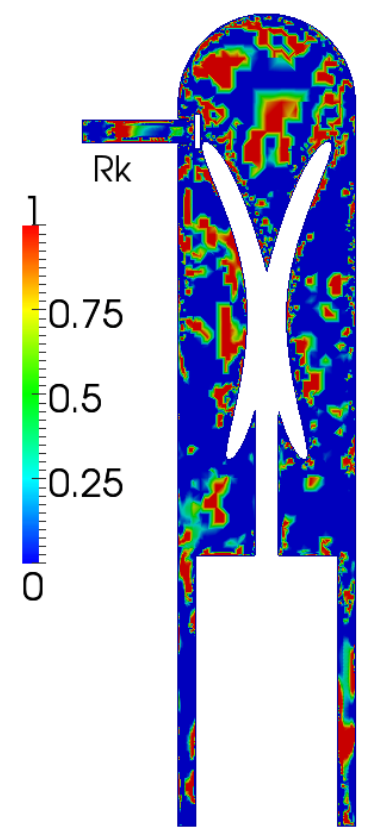

Figure 38: 075 Configuration $R_{k}$ Plot

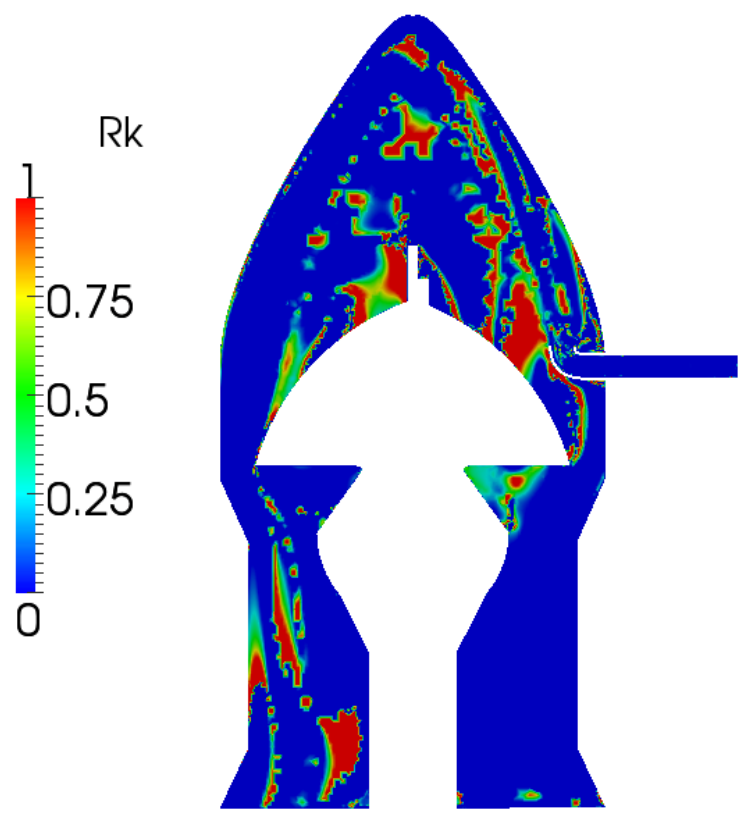

Figure 39: 35 Configuration $R_{k}$ Plot 


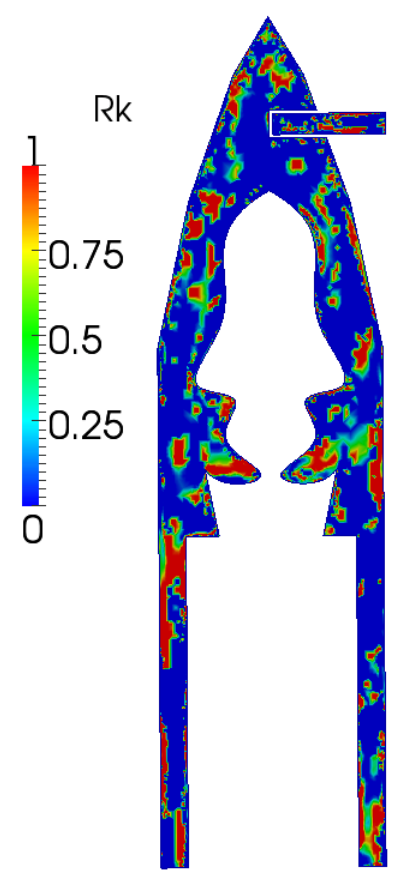

Figure 40: 55 Configuration $R_{k}$ Plot

The solution is not monotonic or divergent. The solution's residuals showed fully converged and well behaved for all models. The solution is actually oscillatory at all points but is being miss represented as monotonic or divergent depending on where the points were taken on the oscillatory curve. This is still an area of discussion [15], [39], [40], [41]. It is extremely difficult to achieve the asymptotic range for a complex problem [15]. This could also be attributed to non-uniform mesh refinement [42]. The proposed methodology is to treat the grid as a separate oscillatory parameter as shown in the next section. 


\subsection{Proposed CFD Uncertainty Method Compared to Exact Solution -Laminar Flow Between Parallel, Stationary Plates}

The uncertainty can be calculated by expanding equation 13 for pressure, density, numerical (grid), and solver.

$$
\begin{gathered}
u_{\text {val }}=k *\left(\left(\left(\frac{\partial V}{\partial \text { pressure }}\right)^{2} B_{\text {pressure }}^{2}\right)+\left(\left(\frac{\partial V}{\partial r h o}\right)^{2} B_{\text {rho }}^{2}\right)+\right. \\
\left.\left(\left(\frac{\partial V}{\partial \text { num }}\right)^{2} B_{\text {num }}^{2}\right)+\left(\left(\frac{\partial V}{\partial \text { solver }}\right)^{2} B_{\text {solver }}^{2}\right)++\left(\left(\frac{\partial V}{\partial \text { velocity }}\right)^{2} B_{\text {velocity }}^{2}\right)\right)^{1 / 2}
\end{gathered}
$$

The proposed method is to calculate the uncertainty as an oscillatory input parameter and multiply by the appropriate Student-T k-factor.

For Numerical, three grids were used and the t value of 2.92 .

$$
\begin{gathered}
u_{v a l}=2.92 *\left(\left(\left(\frac{\partial V}{\partial n u m}\right)^{2} B_{\text {num }}^{2}\right)\right)^{1 / 2} \\
u_{v a l}=2.92 *\left|\frac{1}{2}\left(S_{U}-S_{L}\right)\right|
\end{gathered}
$$

The centerline velocity was chosen as an example to plot, however at all points the uncertainty bands always encompass the exact solution. 


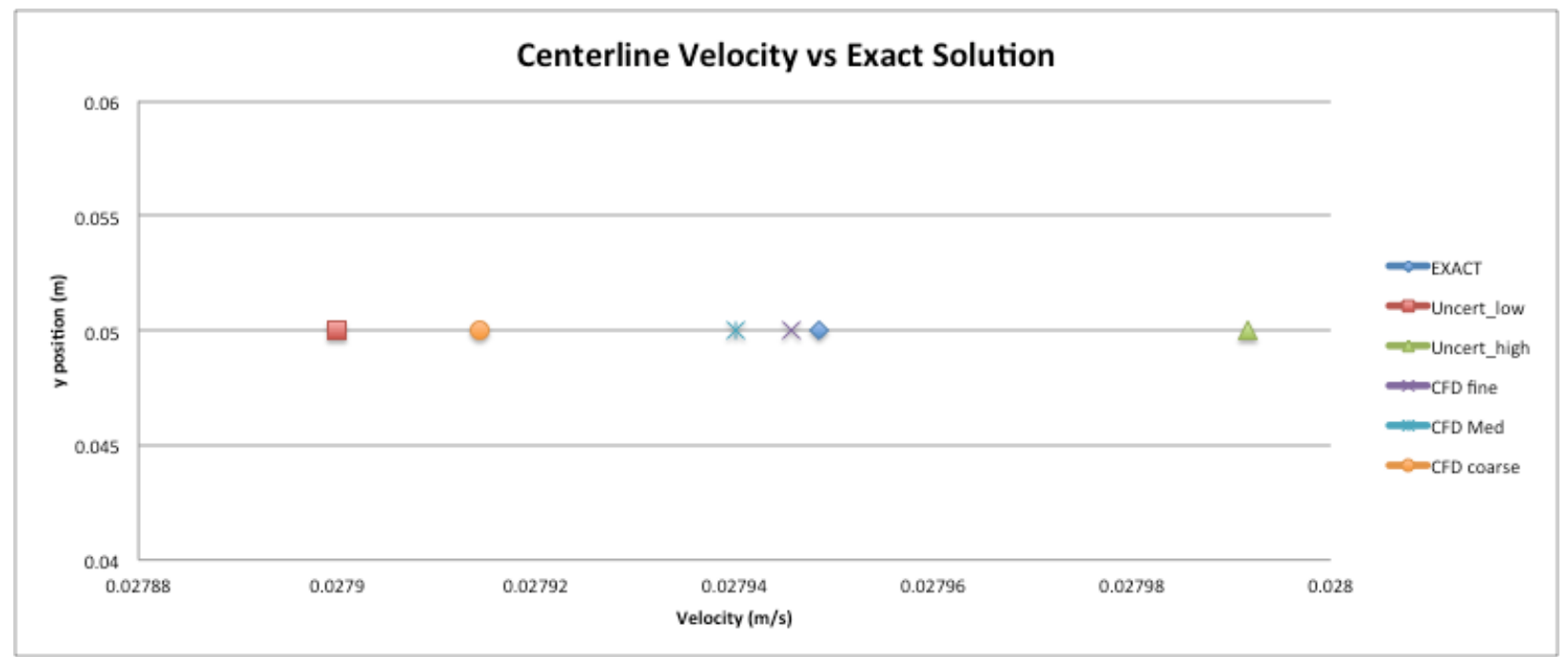

Figure 41: Exact Solution vs. CFD with Uncertainty (Centerline Velocity) - Grid

If there is also a variation in the inlet velocity due to a tolerance or known bias, run the model at the low and high limits and use a new t-value of 2.132, which corresponds to five cases. The five cases would be three for grids and two for flow rates. A five percent variation in inlet velocity was chosen for this example.

$$
\begin{gathered}
u_{\text {val }}=2.132 *\left(\left(\left(\frac{\partial V}{\partial \text { num }}\right)^{2} B_{\text {num }}^{2}\right)+\left(\left(\frac{\partial V}{\partial \text { velocity }}\right)^{2} B_{\text {velocity }}^{2}\right)\right)^{1 / 2} \\
u_{\text {val }}=2.132 *\left|\frac{1}{2}\left(S_{U}-S_{L}\right)\right|
\end{gathered}
$$




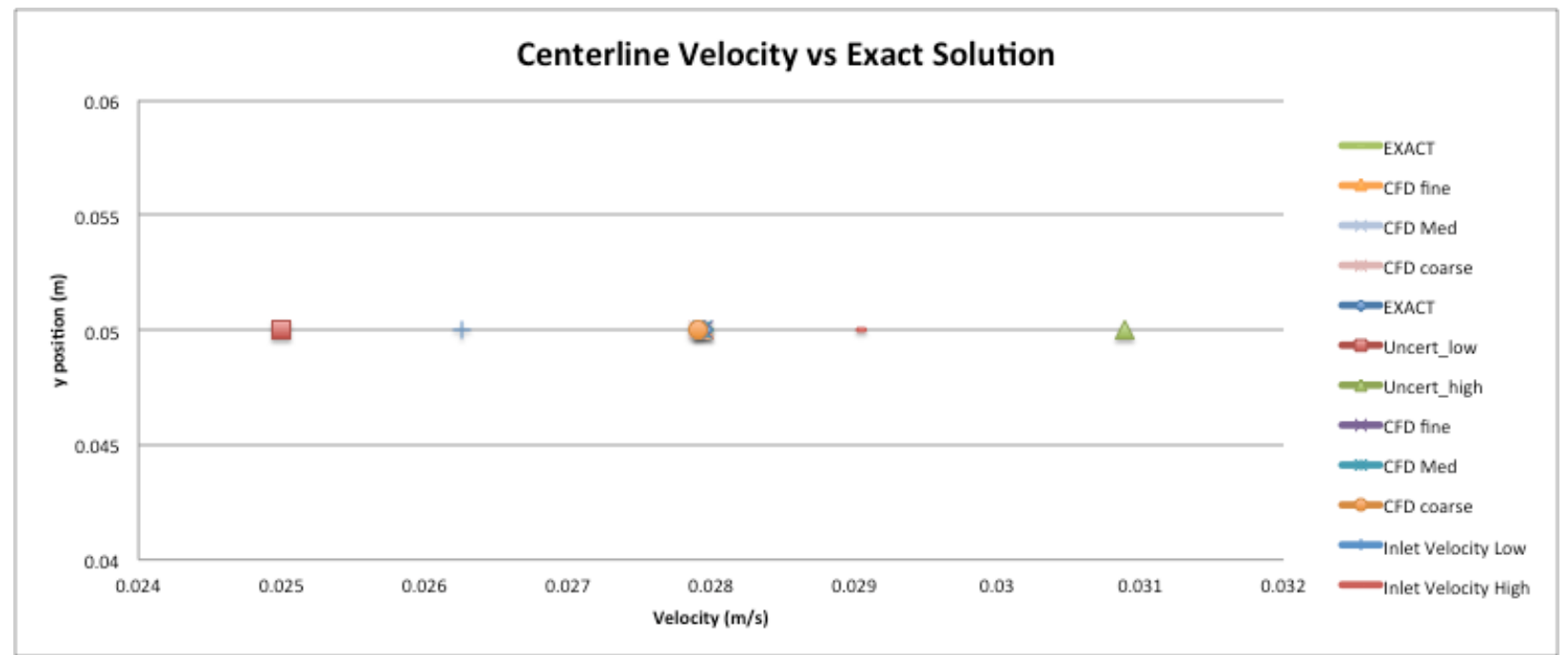

Figure 42: Exact Solution vs. CFD with Uncertainty (Centerline Velocity) - Grid and Inlet Velocity

Also to include the outlet pressure boundary condition, run the model at the low and high known bias or tolerances and use a new t-value of 1.943, which corresponds to seven cases. The seven cases would be three for grid, two for flow rate, and two for pressure outlet boundary condition.

$$
\begin{gathered}
u_{\text {val }}=1.943 *\left(\left(\left(\frac{\partial V}{\partial \text { num }}\right)^{2} B_{\text {num }}^{2}\right)+\left(\left(\frac{\partial V}{\text { dvelocity }}\right)^{2} B_{\text {velocity }}^{2}\right)+\right. \\
\left.\left(\left(\frac{\partial V}{\text { opressure }}\right)^{2} B_{\text {pressure }}^{2}\right)\right)^{1 / 2} \\
u_{\text {val }}=1.943 *\left|\frac{1}{2}\left(S_{U}-S_{L}\right)\right|
\end{gathered}
$$




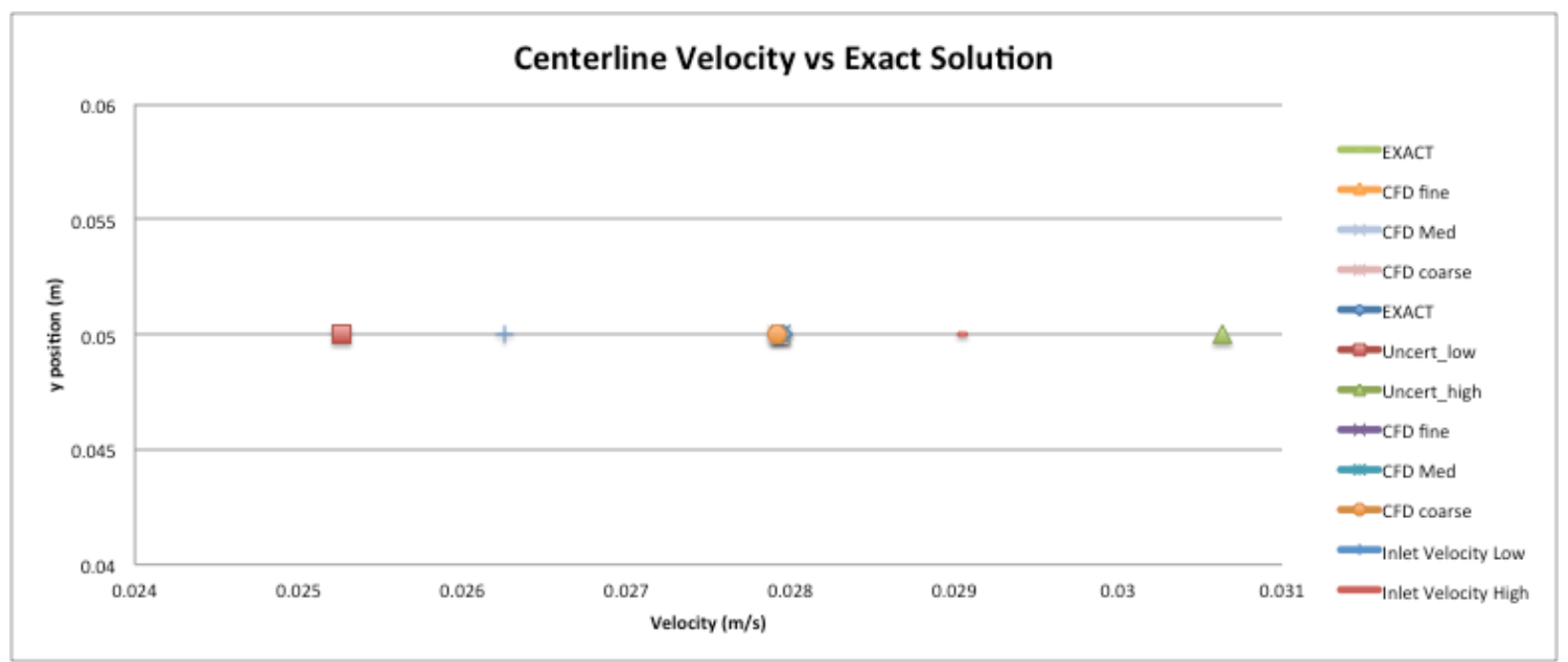

Figure 43: Exact Solution vs. CFD with Uncertainty (Centerline Velocity) - Grid, Inlet Velocity, and Outlet Pressure

To account for the variation in fluid properties, the kinematic viscosity for air between 0 and 100 degrees Celsius is $13.6 \times 10^{-6}$ to $23.06 \times 10^{-6}$. The model was run at these limits to account for the possible variation in fluid properties and a new value of $t=1.86$ was chosen, which corresponds to the nine cases.

$$
\begin{gathered}
u_{\text {val }}=1.86 *\left(\left(\left(\frac{\partial V}{\partial \text { num }}\right)^{2} B_{\text {num }}^{2}\right)+\left(\left(\frac{\partial V}{\partial \text { velocity }}\right)^{2} B_{\text {velocity }}^{2}\right)+\right. \\
\left.\left(\left(\frac{\partial V}{\partial \text { pressure }}\right)^{2} B_{\text {pressure }}^{2}\right)++\left(\left(\frac{\partial V}{\partial r h o}\right)^{2} B_{\text {rho }}^{2}\right)\right)^{1 / 2} \\
u_{\text {val }}=1.86 *\left|\frac{1}{2}\left(S_{U}-S_{L}\right)\right|
\end{gathered}
$$




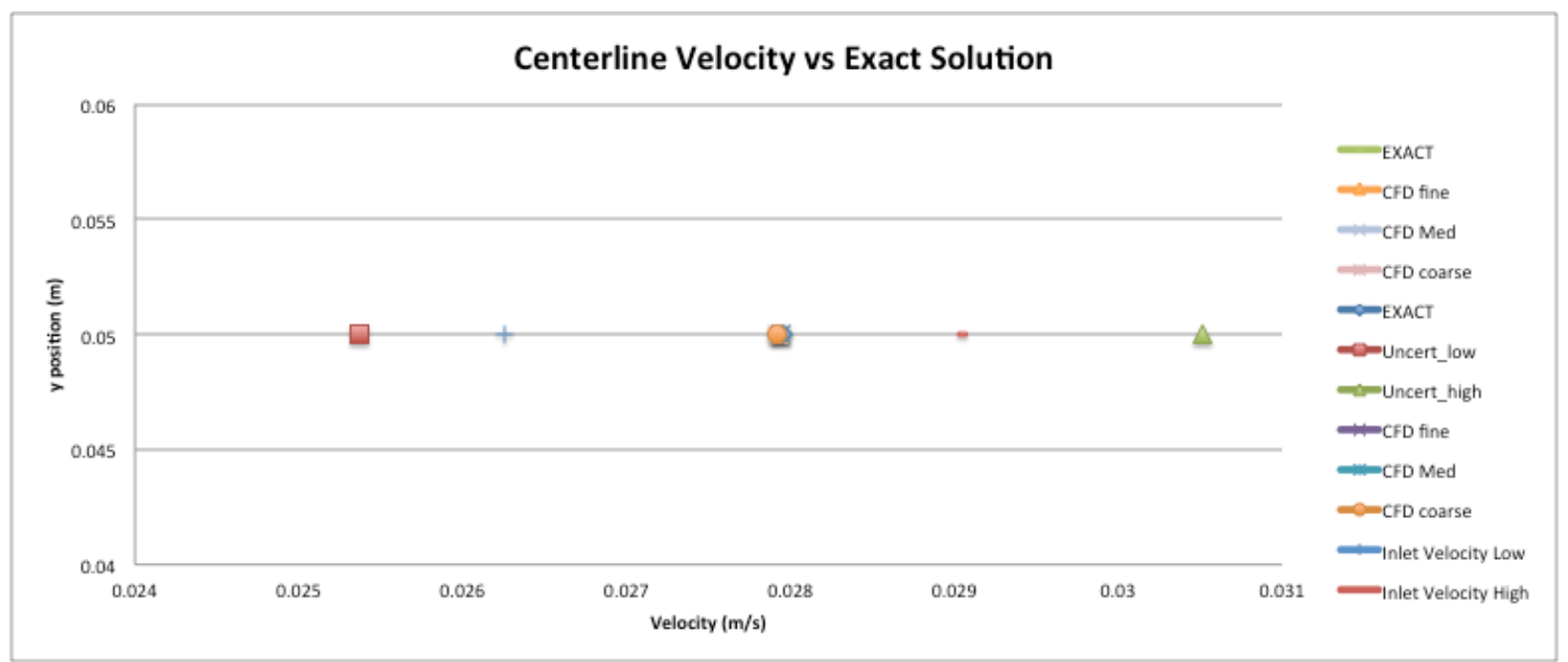

Figure 44: Exact Solution vs. CFD with Uncertainty (Centerline Velocity) - Grid, Inlet Velocity, Outlet Pressure, and Density

Fluent has been used to calculate the results above; we also consider the solver as an input to the model. To account for the variation in the solver, the model was run in OpenFOAM. The $t$ value was updated to 1.833 because the numbers of cases are ten.

$$
\begin{gathered}
u_{\text {val }}=1.833 *\left(\left(\left(\frac{\partial V}{\partial n u m}\right)^{2} B_{\text {num }}^{2}\right)+\left(\left(\frac{\partial V}{\partial \text { velocity }}\right)^{2} B_{\text {velocity }}^{2}\right)+\right. \\
\left.\left(\left(\frac{\partial V}{\partial \text { pressure }}\right)^{2} B_{\text {pressure }}^{2}\right)++\left(\left(\frac{\partial V}{\partial r h o}\right)^{2} B_{\text {rho }}^{2}\right)+\left(\frac{\partial V}{\partial \text { solver }}\right)^{2} B_{\text {solver }}^{2}\right)^{1 / 2} \\
u_{\text {val }}=1.833 *\left|\frac{1}{2}\left(S_{U}-S_{L}\right)\right|
\end{gathered}
$$




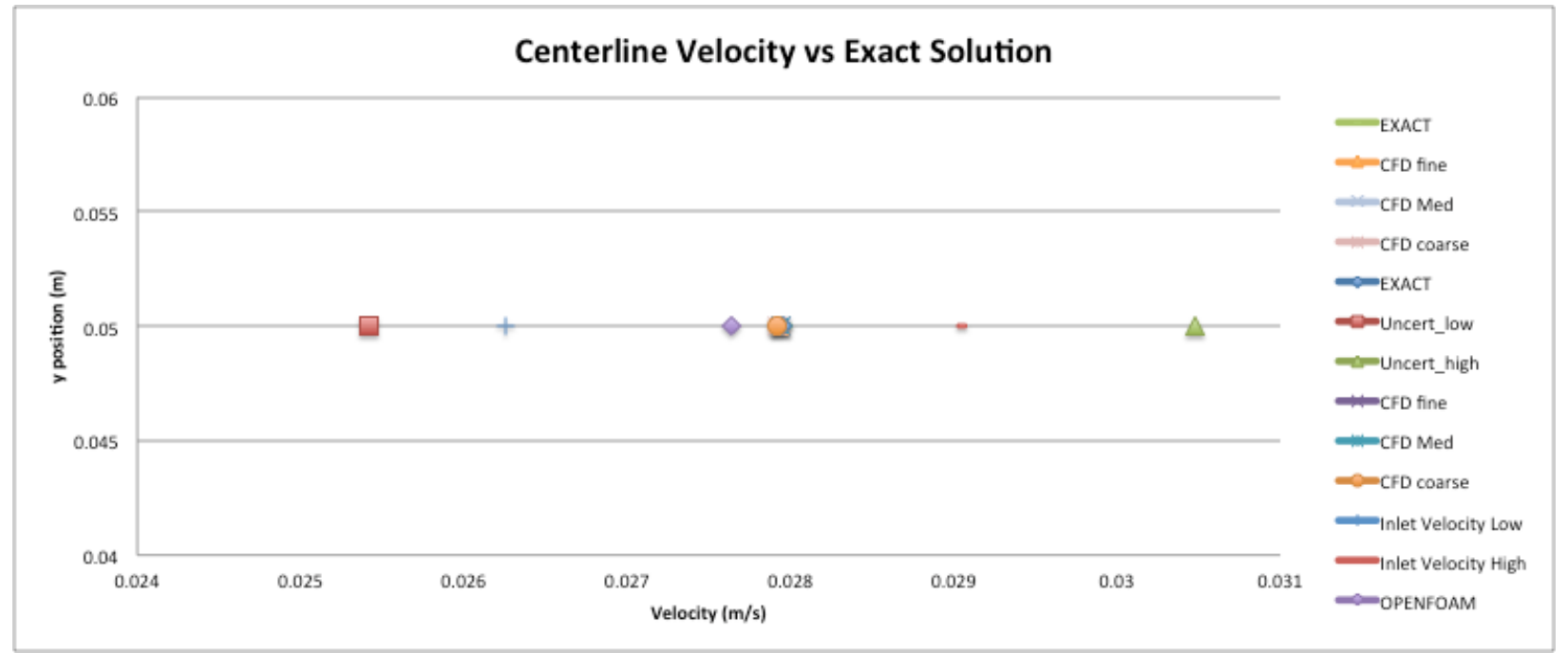

Figure 45: Exact Solution vs. CFD with Uncertainty (Centerline Velocity) - Grid, Inlet Velocity, Outlet Pressure, Density, and Solver

Figure 46 is a plot of all the CFD cases, uncertainty, and an exact comparison.

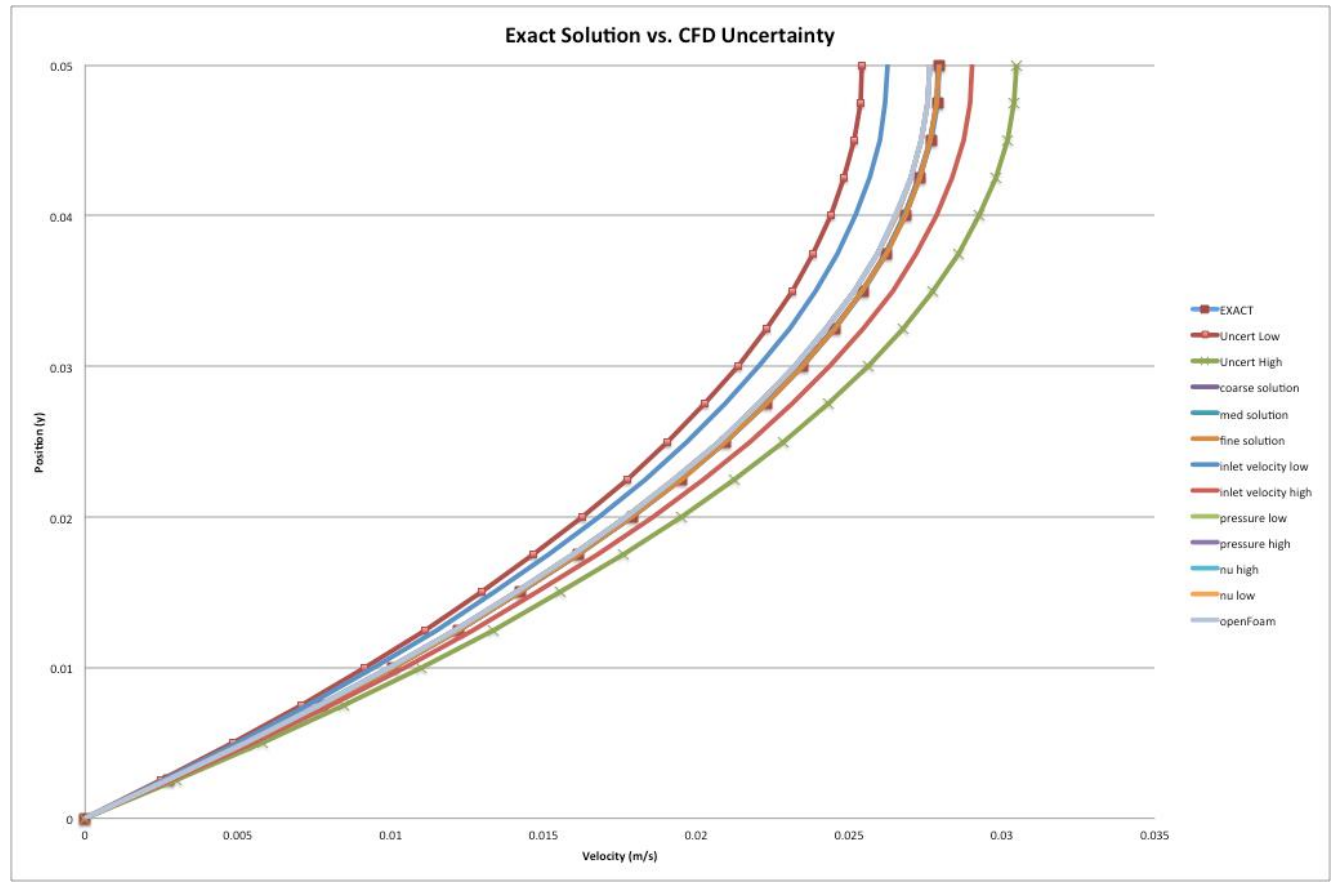

Figure 46: Exact Solution vs. CFD with Uncertainty (Parallel Plates - Half of Domain) - Grid, Inlet Velocity, Outlet Pressure, Density, and Solver 
It can be concluded by treating all inputs to a CFD model as oscillatory uncertainty parameters coupled with the Student-T distribution can supply an uncertainty estimate that encompasses the exact solution for the case considered above (fully developed, laminar, flow between stationary parallel plates). To summarize the approach and general idea, there is a standard [14] for calculating verification and validation of CFD using a combined numerical and experimental data. The approach described above is a way to estimate the uncertainty of a model if test data is not available. An analyst should make use of all available test data or data which can be funded and use the ASME standard. However, if test data is missing or not attainable, the method described makes assumptions about each CFD solution belonging to an underlying Student-T distribution and a corresponding uncertainty can be estimated for a selected confidence interval.

This method can be contrasted with traditional uncertainty analysis of the exact solution. The exact solution for the velocity profile between parallel, stationary plates is shown in equation ( 29 ) .

Traditional uncertainty analysis of this equation can be calculated for the parameters considered ( $5 \%$ uncertainty in $\frac{\partial P}{\partial x}$ and $\mu$ ). This should provide a nearly identical result as compared to the numerical method described above.

$$
u_{u}=\left(\left(\left(\frac{\partial u}{\partial \mu}\right)^{2} B_{\mu}^{2}\right)+\left(\left(\frac{\partial u}{\partial \frac{\partial P}{\partial x}}\right)^{2} B_{\frac{\partial P}{\partial x}}^{2}\right)\right)^{1 / 2}
$$

Calculating the partial derivatives: 


$$
\begin{gathered}
\frac{\partial u}{\partial \mu}=-\frac{\partial P}{\partial x} * y *(y-a) /(2 \mu) \\
\frac{\partial u}{\partial \frac{\partial P}{\partial x}}=\frac{a^{2}}{2 \mu}\left[\left(\frac{y}{a}\right)^{2}-\left(\frac{y}{a}\right)\right]
\end{gathered}
$$

Plugging the partial derivatives into equation ( 42 ). The equation becomes the exact uncertainty for a $5 \%$ variation in $\frac{\partial P}{\partial x}$ and $\mu$ as was the numerical method described above.

$$
\begin{gathered}
u_{u}=\left(\left(\left(-\frac{\partial P}{\partial x} * y *(y-a) /(2 \mu)\right)^{2} B_{\mu}^{2}\right)\right. \\
\left.+\left(\left(\frac{a^{2}}{2 \mu}\left[\left(\frac{y}{a}\right)^{2}-\left(\frac{y}{a}\right)\right]\right)^{2} B_{\frac{\partial P}{\partial x}}^{2}\right)\right)^{1 / 2}
\end{gathered}
$$

Figure 47 and Figure 48 was created to show the uncertainty and the exact solution with uncertainty bands. 


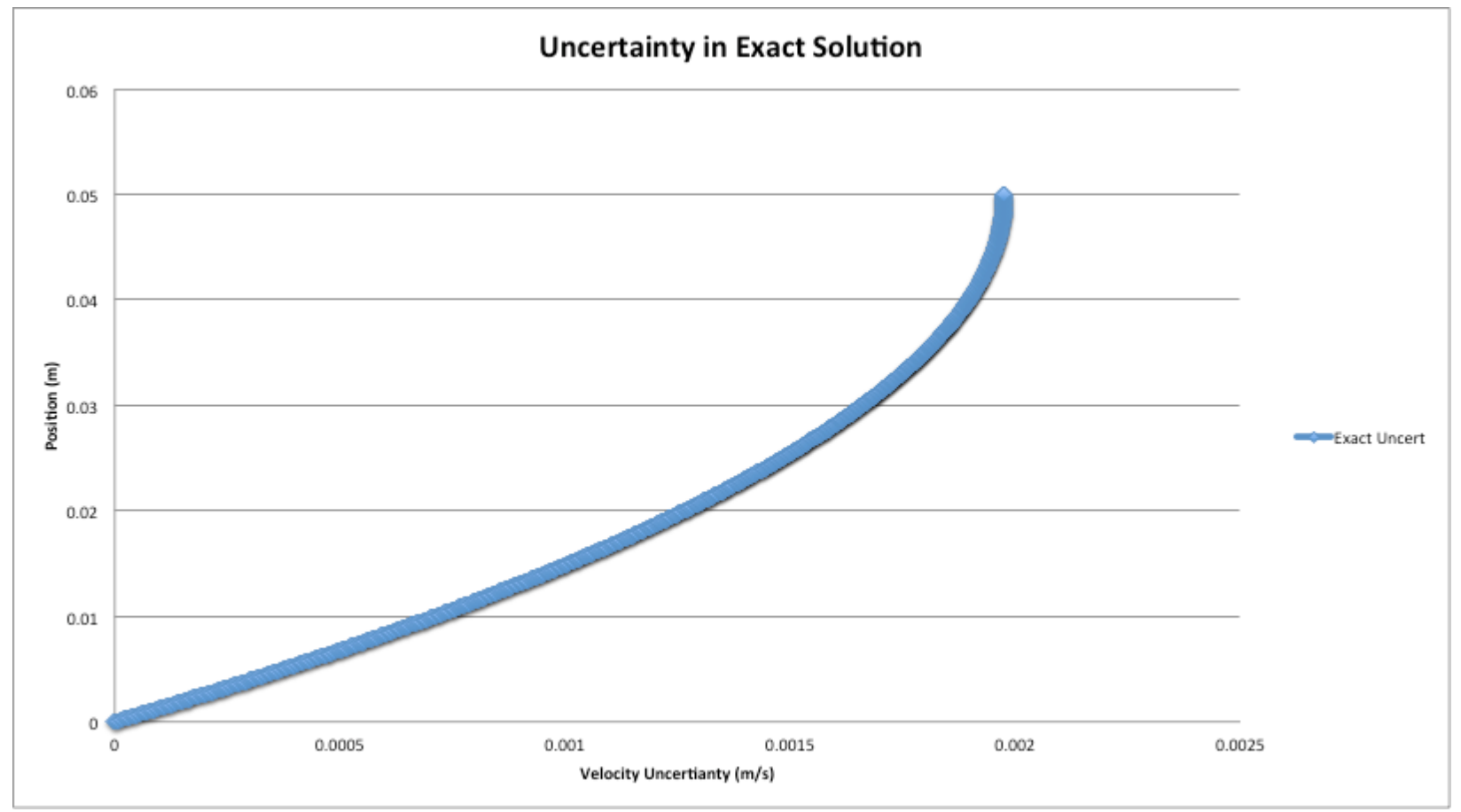

Figure 47: Exact Solution Uncertainty

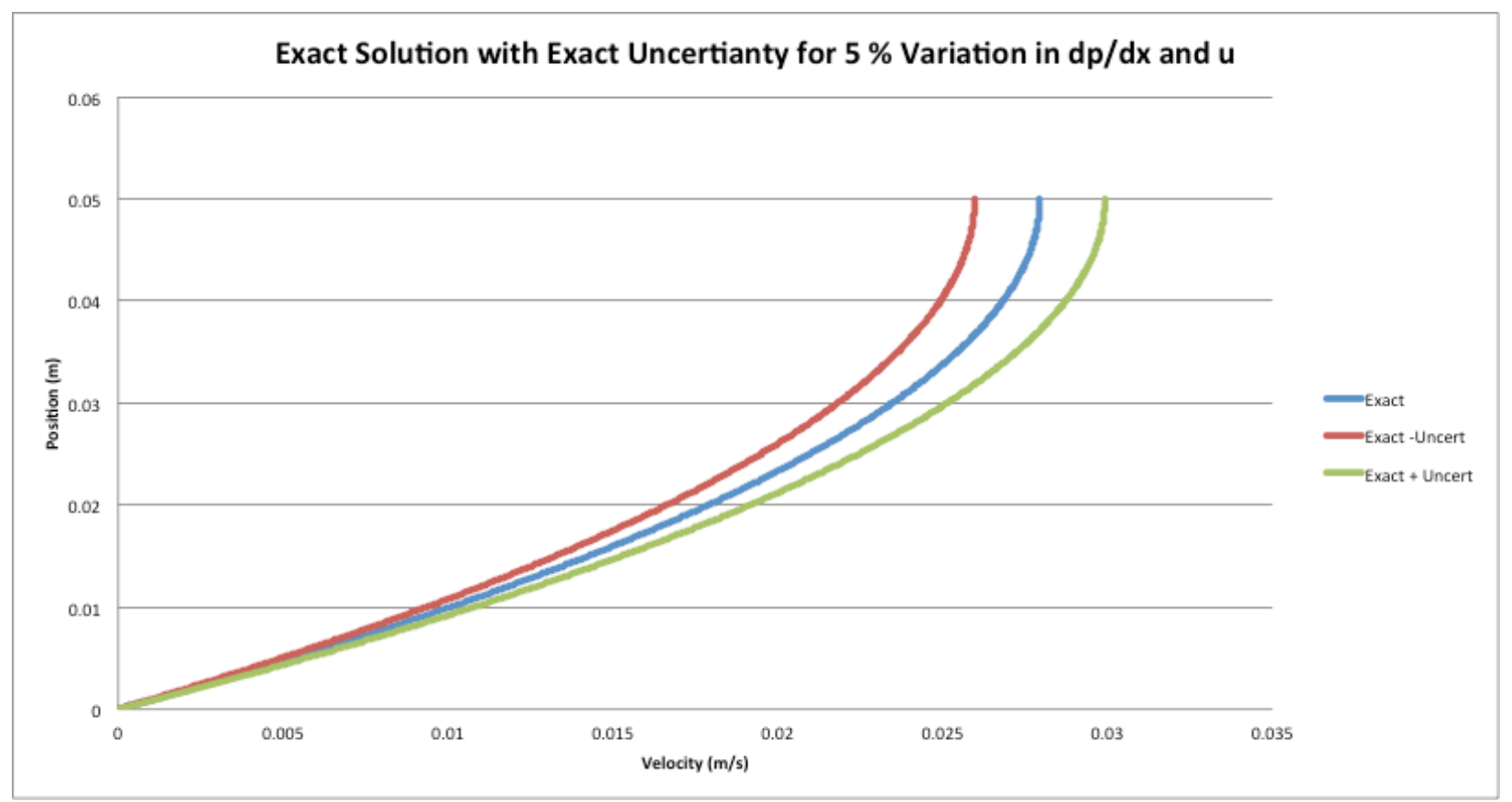

Figure 48: Exact Solution Uncertainty 
To contrast the methods, the numerically derived uncertainty in equations 26, 27 and Figure 43 are compared to the traditional exact derivation of uncertainty as shown in Figure 50.

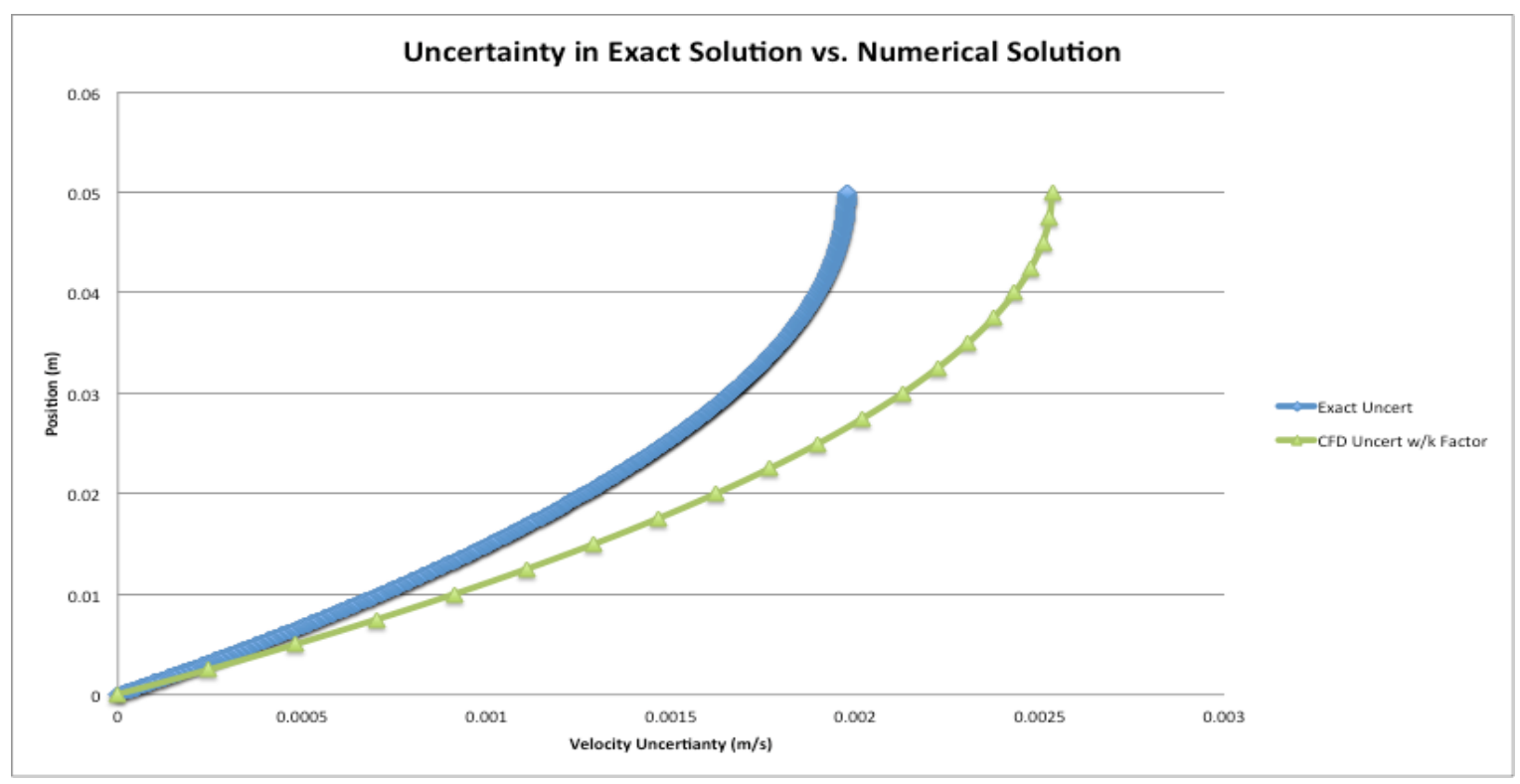

Figure 49: Exact Solution Uncertainty vs. Numerically Derived Uncertainty

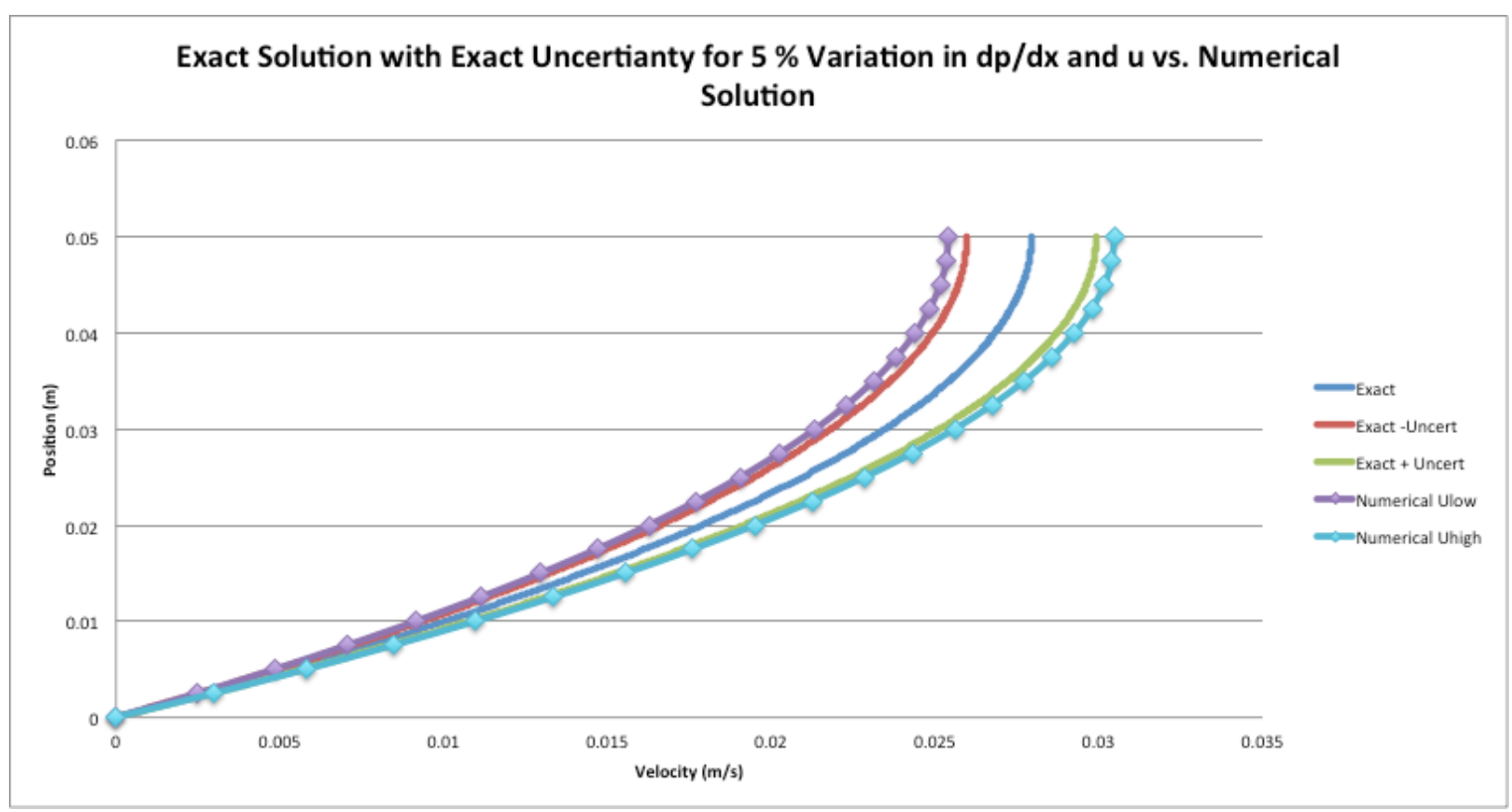

Figure 50: Exact Solution with Uncertainty vs. Numerically Derived Uncertainty 
The numerically derived uncertainty method using the student $\mathrm{t}$ value of $\mathrm{k}=1.833$ is conservative and over-predicts the uncertainty. Also important to note is a k-value of 1.43 matches the two methods as shown in Figure 52. $\mathrm{K}=1.43$ is an interesting result and there may be some correlation between this number and other distributions.

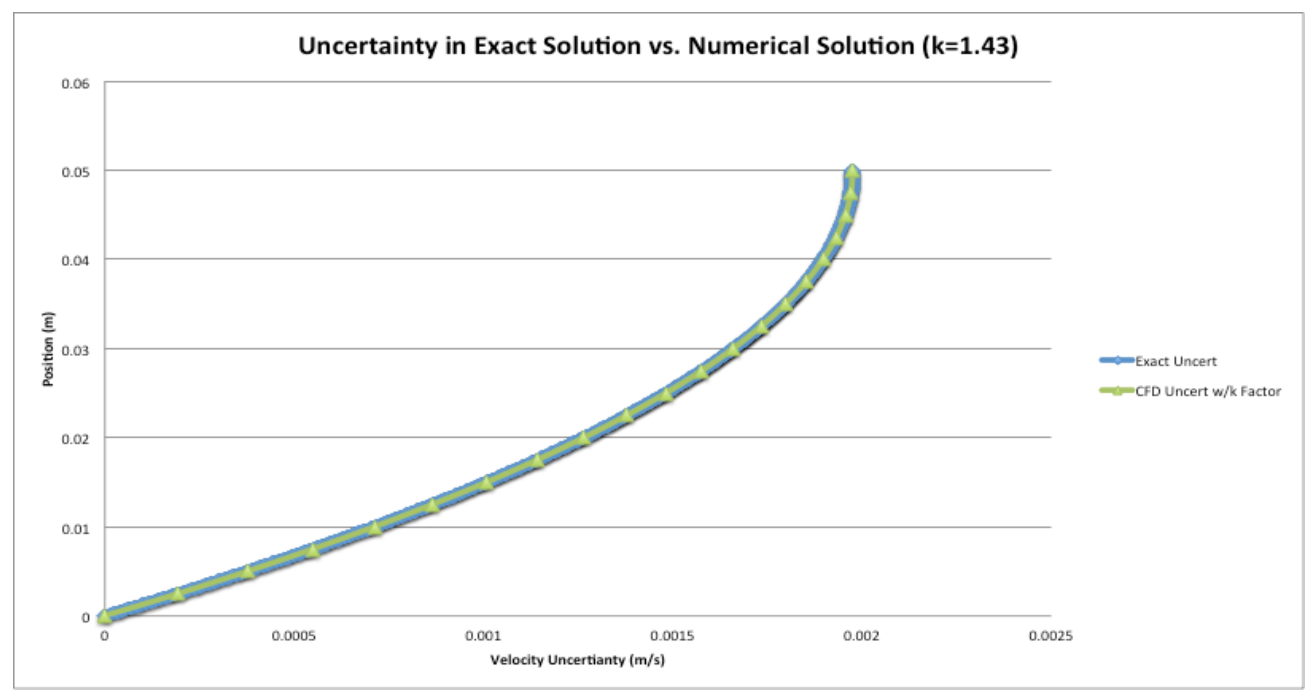

Figure 51: Exact Solution Uncertainty vs. Numerically Derived Uncertainty (with k=1.43)

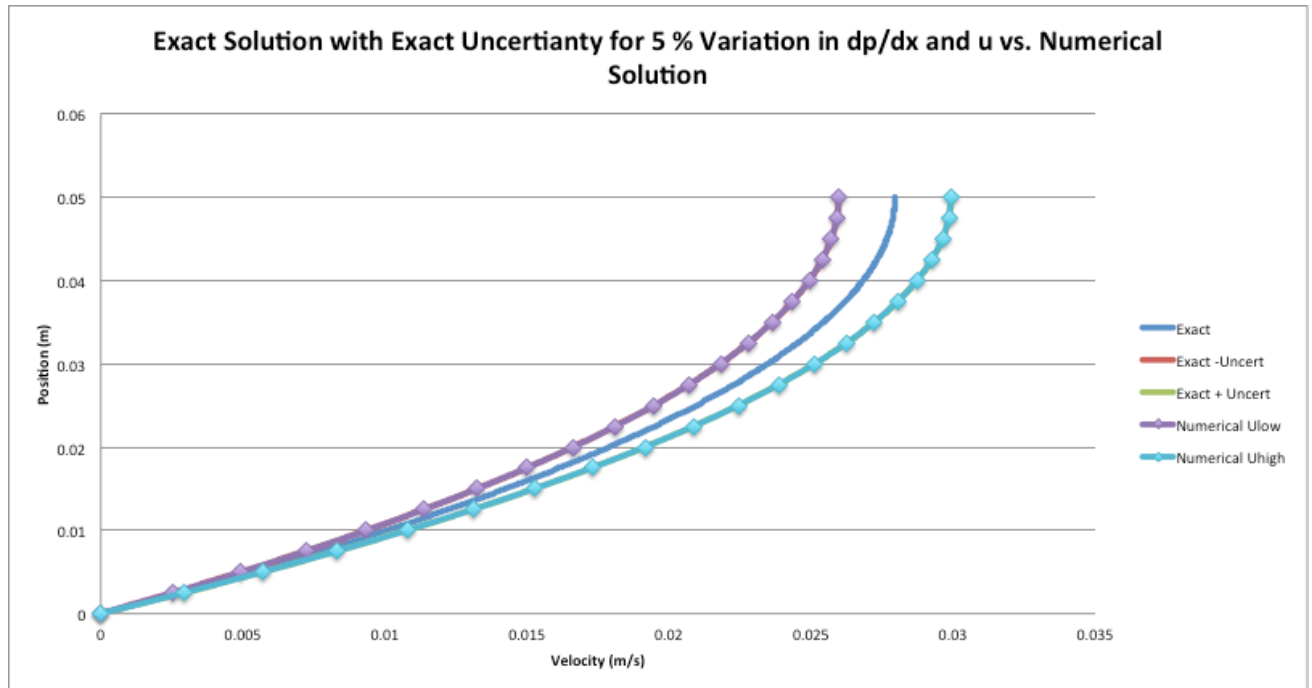

Figure 52: Exact Solution Uncertainty vs. Numerically Derived Uncertainty (with k=1.43) 


\subsection{Proposed CFD Uncertainty Method Applied to Heat Transfer over a Flat Plate}

The data in this section was published by the author in DFD13-2013-00087 [43]. Cornell University has posted a FLUENT example problem of forced convection over a flat plate [44]. This example problem is a good demonstration of traditional uncertainty analysis with experimental data verses the proposed methodology using CFD.

\subsubsection{Correlation Uncertainty Calculation}

Different correlations have been derived for this simple problem based off experimental data. They are generally in the form of equation ( 46 ).

$$
\frac{h L}{k}=c\left(\frac{\rho V L}{\mu}\right)^{4 / 5}
$$

The uncertainty in the heat transfer coefficient can be calculated as follows.

$$
h=c\left(\frac{\rho V L}{\mu}\right)^{4 / 5} \frac{k}{L}
$$

Differentiating with respect to each of the variables:

$$
\begin{aligned}
& \frac{d h}{d V}=\frac{4 c k \rho}{5 \mu\left(\frac{L V \rho}{\mu}\right)^{\frac{1}{5}}} \\
& \frac{d h}{d \rho}=\frac{4 c k V}{5 \mu\left(\frac{L V \rho}{\mu}\right)^{\frac{1}{5}}}
\end{aligned}
$$




$$
\begin{gathered}
\frac{d h}{d k}=\frac{c}{L}\left(\frac{L V \rho}{\mu}\right)^{4 / 5} \\
\frac{d h}{d \mu}=-\frac{4 C V k \rho}{5 \mu^{2}\left(\frac{L V \rho}{\mu}\right)^{\frac{1}{5}}} \\
\frac{d h}{d L}=\frac{4 C V k \rho}{5 L \mu\left(\frac{L V \rho}{\mu}\right)^{\frac{1}{5}}-\frac{C k\left(\frac{L V \rho}{\mu}\right)^{4 / 5}}{L^{2}}} \\
\frac{d h}{d C}=\frac{k}{L}\left(\frac{\rho V L}{\mu}\right)^{4 / 5}
\end{gathered}
$$

Expanding the uncertainty equation

$$
\begin{gathered}
U_{h}=\left(\left(\left(\frac{\partial h}{\partial V}\right)^{2} B_{V}^{2}\right)+\left(\left(\frac{\partial h}{\partial \rho}\right)^{2} B_{\rho}^{2}\right)+\left(\left(\frac{\partial h}{\partial k}\right)^{2} B_{k}^{2}\right)+\left(\left(\frac{\partial h}{\partial \mu}\right)^{2} B_{\mu}^{2}\right)\right. \\
+\left(\left(\frac{\partial h}{\partial L}\right)^{2} B_{L}^{2}\right)+\left(\left(\frac{\partial h}{\partial C}\right)^{2} P_{C}^{2}\right)+2\left(\frac{\partial h}{\partial \rho}\right)\left(\frac{\partial h}{\partial k}\right) B_{\rho} B_{k} \\
\left.+2\left(\frac{\partial h}{\partial \rho}\right)\left(\frac{\partial h}{\partial \mu}\right) B_{\rho} B_{\mu}+2\left(\frac{\partial h}{\partial k}\right)\left(\frac{\partial h}{\partial \mu}\right) B_{k} B_{\mu}\right)^{1 / 2}
\end{gathered}
$$

Plugging in the partial derivatives 


$$
\begin{aligned}
& U_{h}=\left(\left(\left(\frac{4 c k \rho}{5 \mu\left(\frac{L V \rho}{\mu}\right)^{\frac{1}{5}}}\right)^{2} B_{V}^{2}\right)+\left(\left(\frac{4 c k V}{5 \mu\left(\frac{L V \rho}{\mu}\right)^{\frac{1}{5}}}\right)^{2} B_{\rho}^{2}\right)+\left(\left(\frac{c}{L}\left(\frac{L V \rho}{\mu}\right)^{4 / 5}\right)^{2} B_{k}^{2}\right)\right. \\
& +\left(\left(\frac{\partial h}{\partial \mu}\right)^{2} B_{\mu}^{2}\right)+\left(\left(\frac{4 C V k \rho}{5 L \mu\left(\frac{L V \rho}{\mu}\right)^{\frac{1}{5}}}-\frac{C k\left(\frac{L V \rho}{\mu}\right)^{4 / 5}}{L^{2}}\right)^{2} B_{L}^{2}\right) \\
& +\left(\left(\frac{k}{L}\left(\frac{\rho V L}{\mu}\right)^{4 / 5}\right)^{2} P_{C}^{2}\right)+2\left(\frac{4 c k V}{5 \mu\left(\frac{L V \rho}{\mu}\right)^{\frac{1}{5}}}\right)\left(\frac{c}{L}\left(\frac{L V \rho}{\mu}\right)^{4 / 5}\right) B_{\rho} B_{k} \\
& +2\left(\frac{4 c k V}{5 \mu\left(\frac{L V \rho}{\mu}\right)^{\frac{1}{5}}}\right)\left(-\frac{4 C V k \rho}{5 \mu^{2}\left(\frac{L V \rho}{\mu}\right)^{\frac{1}{5}}}\right) B_{\rho} B_{\mu} \\
& \left.+2\left(\frac{c}{L}\left(\frac{L V \rho}{\mu}\right)^{4 / 5}\right)\left(-\frac{4 C V k \rho}{5 \mu^{2}\left(\frac{L V \rho}{\mu}\right)^{\frac{1}{5}}}\right) B_{k} B_{\mu}\right)^{1 / 2}
\end{aligned}
$$

Using the variables and bias errors in Table 8 and Table 9 to numerically evaluating the expression in equation ( 55 ) results in the following. The c values are shown below for a couple of correlations.

Table 8: c Value

\begin{tabular}{|c|c|}
\hline$\underline{\mathbf{c}}$ & \\
\hline Seban \& Doughty & 0.0236 \\
\hline Jakob & 0.024 \\
\hline Sugawara & 0.023 \\
\hline $\begin{array}{c}\text { Fundamentals of Heat and Mass } \\
\text { Transfer }\end{array}$ & 0.0296 \\
\hline & 0.0263 \\
\hline c middle & 0.0033 \\
\hline c uncert (random) & \\
\hline
\end{tabular}


Table 9: Variables and Bias Errors

\begin{tabular}{|c|c|}
\hline Variable & Bias \\
\hline Velocity, V & $3 \%$ \\
\hline Density, rho & $3 \%$ \\
\hline Thermal Conductivity, $\mathrm{k}$ & $3 \%$ \\
\hline Viscosity, mu & $3 \%$ \\
\hline
\end{tabular}

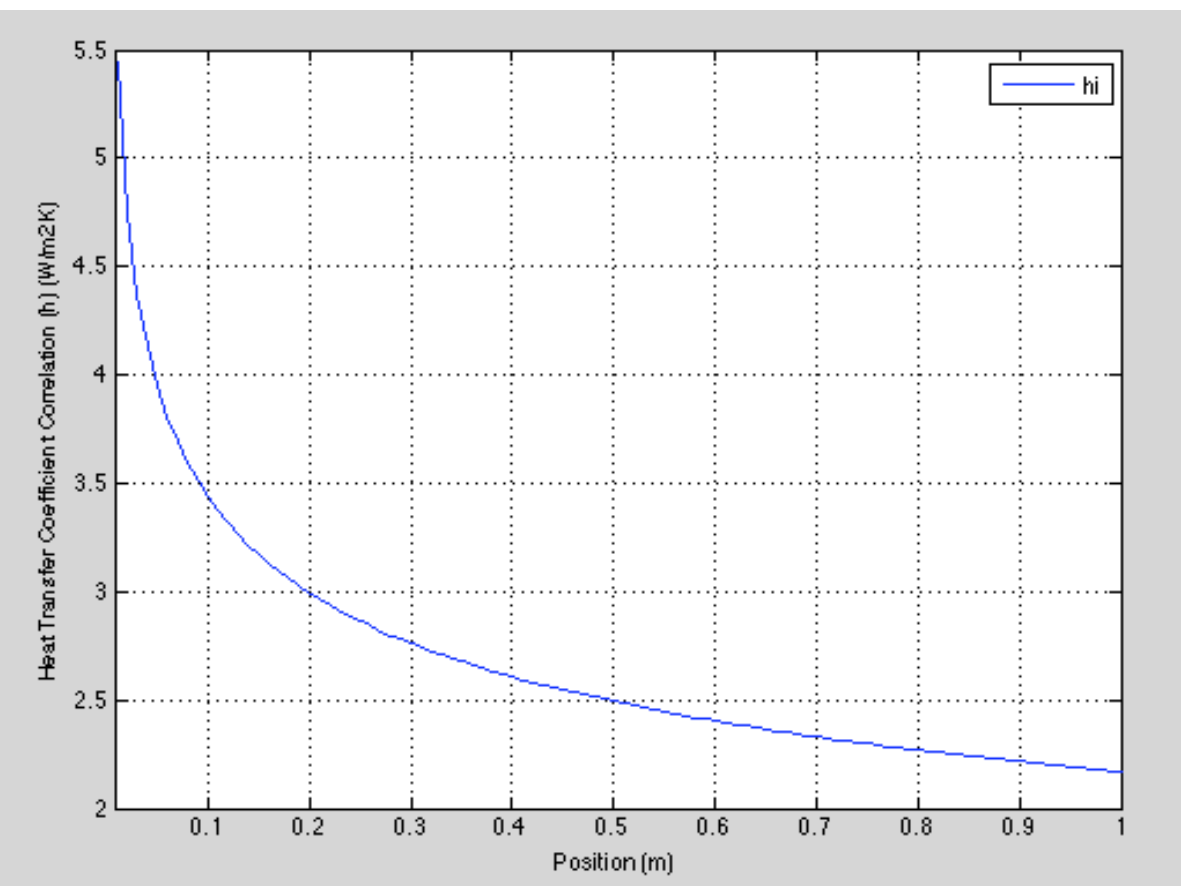

Figure 53: Heat Transfer Coefficient - Correlation 


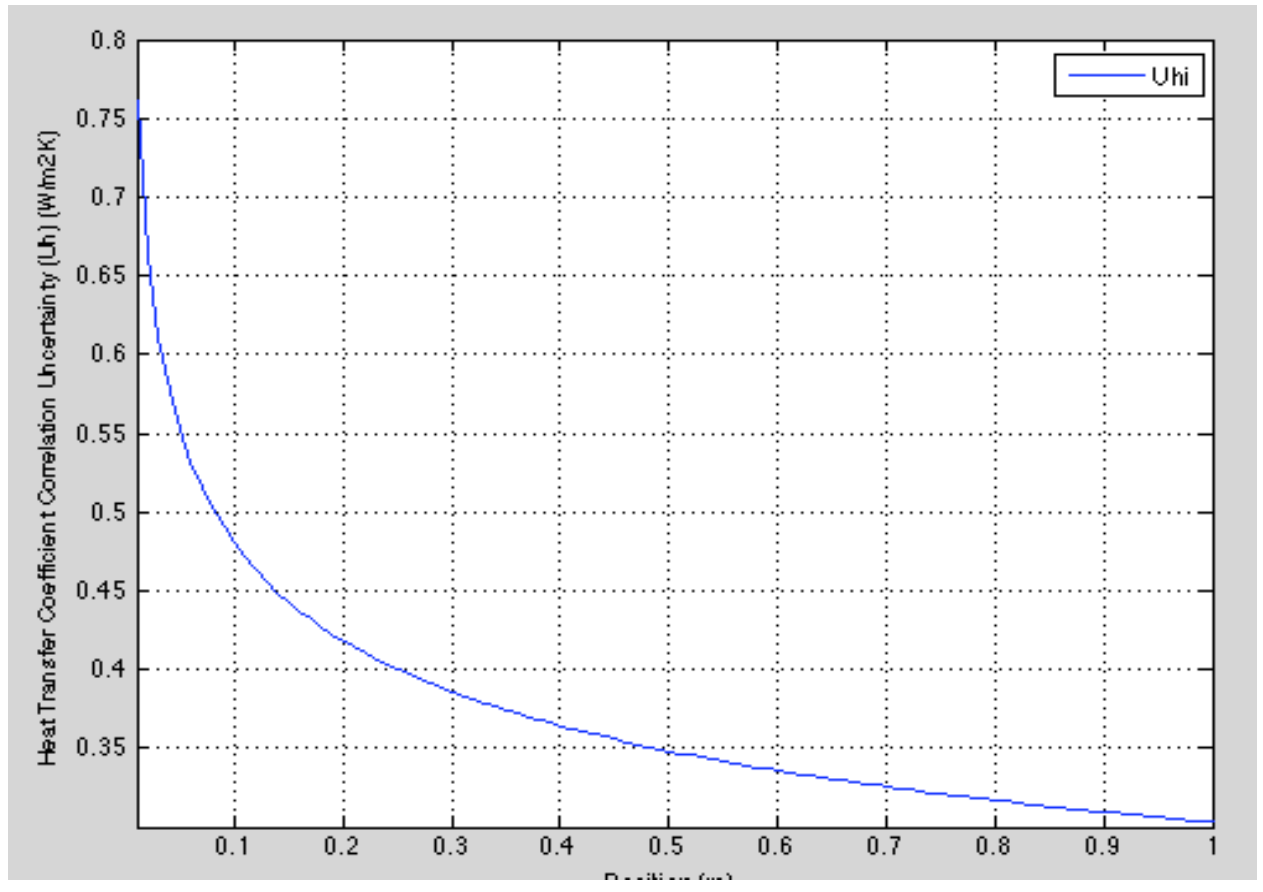

Figure 54: Heat Transfer Coefficient and Uncertainty - Correlation

\subsubsection{CFD Uncertainty Calculation}

To calculate the uncertainty of heat transfer over a flat plate using CFD, the proposed methodology was used as follows.

$$
\begin{gathered}
U_{h}=\left(\left(\left(\frac{\partial h}{\partial V}\right)^{2} B_{V}^{2}\right)+\left(\left(\frac{\partial h}{\partial \rho}\right)^{2} B_{\rho}^{2}\right)+\left(\left(\frac{\partial h}{\partial k}\right)^{2} B_{k}^{2}\right)+\left(\left(\frac{\partial h}{\partial \mu}\right)^{2} B_{\mu}^{2}\right)+\left(\left(\frac{\partial h}{\partial L}\right)^{2} B_{L}^{2}\right)\right. \\
+\left(\left(\frac{\partial h}{\partial C}\right)^{2} P_{C}^{2}\right)+2\left(\frac{\partial h}{\partial \rho}\right)\left(\frac{\partial h}{\partial k}\right) B_{\rho} B_{k}+2\left(\frac{\partial h}{\partial \rho}\right)\left(\frac{\partial h}{\partial \mu}\right) B_{\rho} B_{\mu} \\
\left.+2\left(\frac{\partial h}{\partial k}\right)\left(\frac{\partial h}{\partial \mu}\right) B_{k} B_{\mu}\right)^{1 / 2}
\end{gathered}
$$




$$
u_{v a l}=1.782 *\left|\frac{1}{2}\left(S_{U}-S_{L}\right)\right|
$$

The following cases were run in FLUENT and shown in Table 10.

Table 10: CFD Cases - Flat Plate HTC

\begin{tabular}{|c|c|}
\hline & CFD Uncertainty Cases \\
\hline 1 & Coarse Grid \\
\hline 2 & Medium Grid \\
\hline 3 & Fine Grid \\
\hline 4 & Velocity Low \\
\hline 5 & Velocity High \\
\hline 6 & Density Low \\
\hline 7 & Density High \\
\hline 8 & Thermal Conductivity High \\
\hline 9 & Thermal Conductivity Low \\
\hline 10 & Viscosity Low \\
\hline 11 & Viscosity High \\
\hline 12 & SA Turbulence Model \\
\hline 13 & kwSST Turbulence Model \\
\hline
\end{tabular}

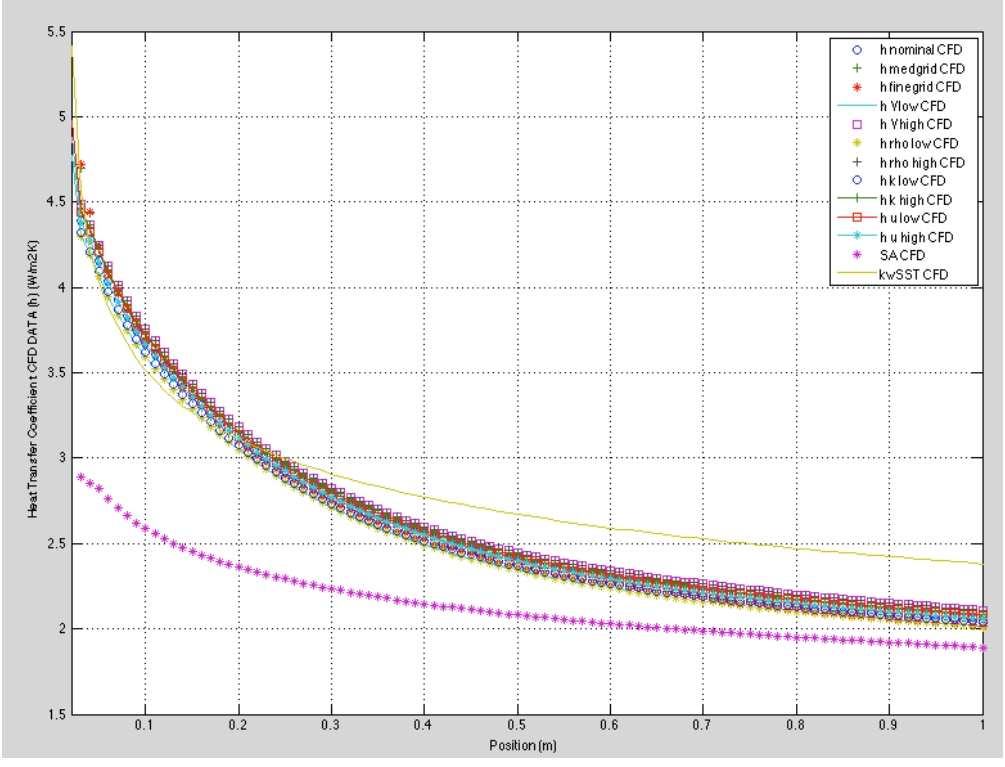

Figure 55: Heat Transfer Coefficient and Uncertainty - CFD Data 


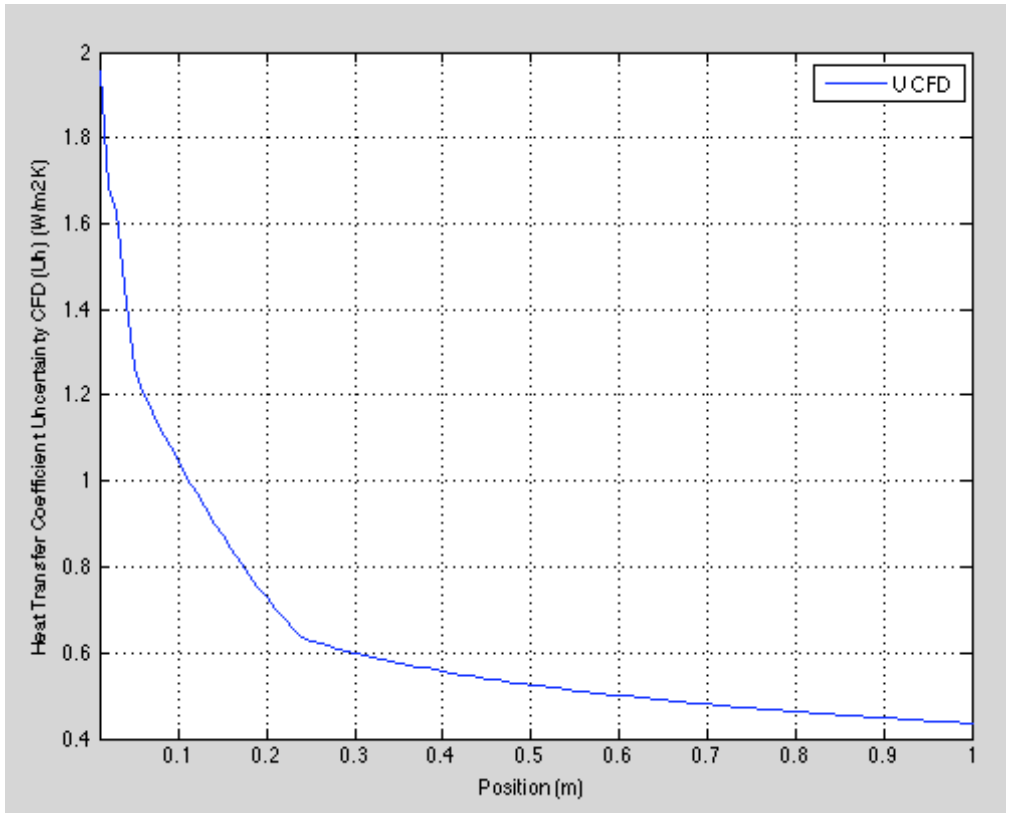

Figure 56: Heat Transfer Coefficient and Uncertainty - CFD Data

\subsubsection{Comparison and Discussion}

To compare the two methods, the results from both cases are plotted together. The CFD uncertainty method is more conservative than the traditional uncertainty analysis using the correlation. 


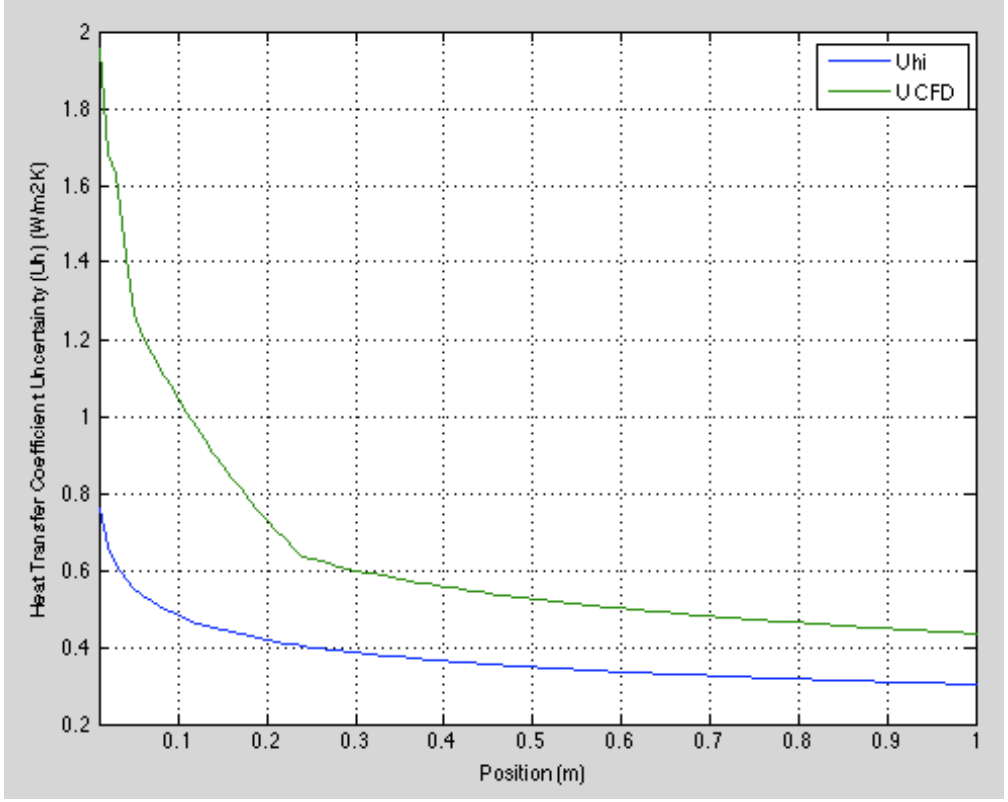

Figure 57: Heat Transfer Coefficient Uncertainty - CFD Data vs. Correlation

Figure 58 is a plot of both the CFD results with uncertainty and the correlation results with uncertainty. The proposed CFD uncertainty method encompasses the correlation results. 


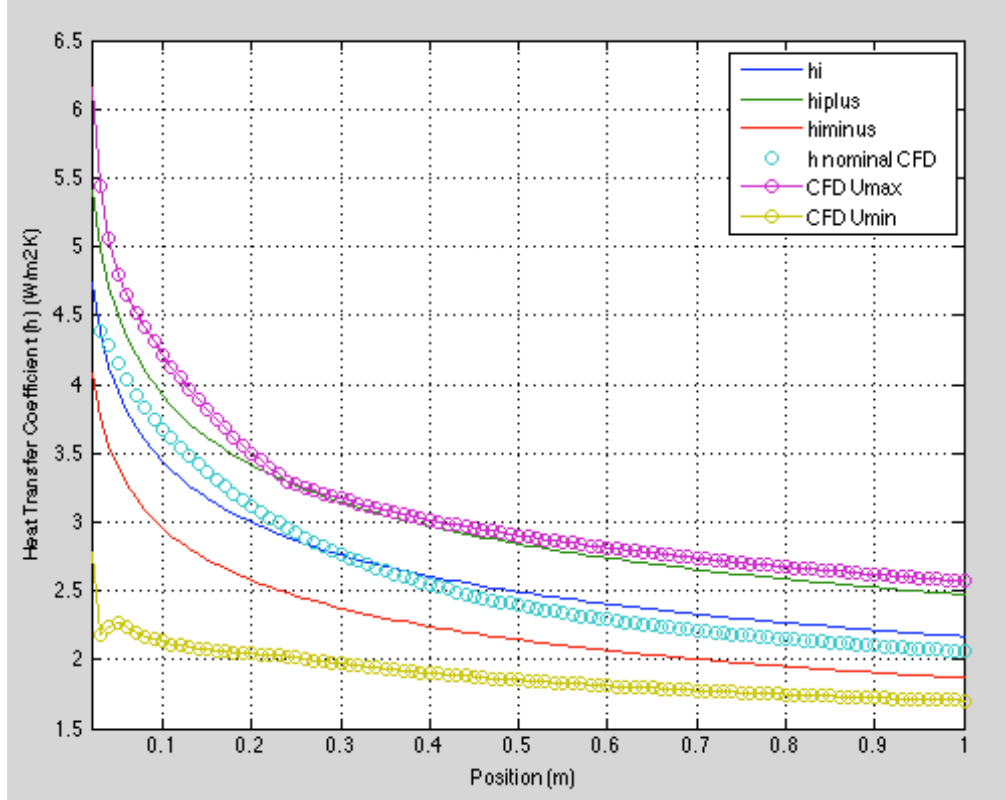

Figure 58: Heat Transfer Coefficient with Uncertainty - CFD Data vs. Correlation

Another interesting item to note is the k-value of 1.2 provides an uncertainty estimate close to the traditional correlation estimate as shown in Figure 59. 


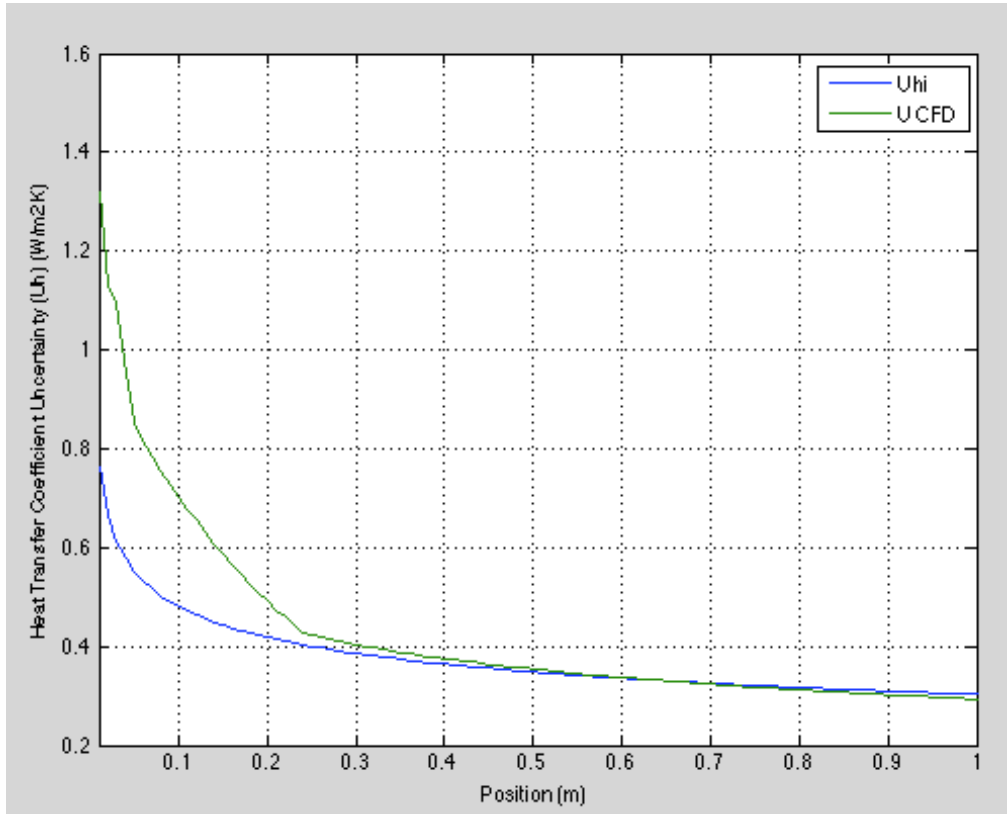

Figure 59: Heat Transfer Coefficient Uncertainty - CFD Data $(\mathrm{k}=1.2)$ vs. Correlation

Another item of interest is the average heat transfer coefficient over the entire flat plate and uncertainty as calculated from each method. The traditional way using the correlation and partial derivatives the average heat transfer coefficient is $2.66+/-0.74\left[\mathrm{~W} / \mathrm{m}^{2} \mathrm{~K}\right]$ and the proposed methodology is $2.66+/-1.39\left[\mathrm{~W} / \mathrm{m}^{2} \mathrm{~K}\right]$.

The work is this chapter was needed before moving into the problem of interest, Spacecraft ECS Systems. The proposed methodology proved accurate for two simple problems, laminar flow between parallel plates and heat transfer over a flat plate. Additionally, the interpolation direction and methodology was developed using the 'linear' option to interpolate all solutions onto the course grid. 


\section{CHAPTER SIX: DEMONSTRATION AND IMPLEMENTATION OF THE PROPOSED CFD UNCERTAINITY METHOD FOR SPACECRAFT ECS SYSTEMS}

The information in this chapter was published by the author in AIAA-2014-0440 [21]. To further elaborate the problem and purpose. There have been few discussions on using Computational Fluid Dynamics (CFD) without experimental validation. Pairing experimental data, uncertainty analysis, and analytical predictions provides a comprehensive approach to verification and is the current state of the art. With pressed budgets, collecting experimental data is rare or nonexistent. This section investigates and proposes a method to perform CFD uncertainty analysis only from computational data. The method uses current CFD uncertainty techniques coupled with the Student-T distribution to predict the velocity magnitude and uncertainty for payload fairing ECS systems. The inputs to the CFD model are varied from a specified tolerance or bias error and the difference in the results are used to estimate the uncertainty. The variation in each input is ranked from least to greatest to determine the order of importance. The results provide a tactic to analytically estimate the uncertainty in a CFD model when experimental data is unavailable.

Chapter 6 is organized by configuration. Chapter 6.1 describes the results of the $0.75 \mathrm{~m}$ configuration, chapter 6.2 the $3.5 \mathrm{~m}$ configuration, and chapter 6.3 the $5.5 \mathrm{~m}$ configuration. Chapter 6.4 demonstrates the proposed methodology as compared to experimental data previously published. The remainder of this section is a summary of the uncertainty calculation and input parameters used for the ECS system analysis. The following uncertainty parameters were considered as shown in Table 11. 
The input parameter uncertainties in the simulations were selected by the physical variables in the problem. To simulate reality using a CFD model, boundary conditions are selected for the appropriate simulation domain. The boundary conditions are physical in nature (ie Pressure, Velocity, Mass Flow Rate) and there is a corresponding bias uncertainty (simulation value contains an offset) and random uncertainty (repeated measurements generally provide a value different from the previous value). The input parameters selected should reflect the boundary conditions and known uncertainties. For example in the ECS spacecraft case, the inlet velocity should be based on the known volumetric flow rate set point. The flow is physically set by selecting (dialing in) an upstream pressure that results in the desired flow rate. The flow rate is being measured and will have an associated bias error. This bias error is usually found in the flow rate manufactures documentation as a known value that contains a bias (for example $+/-5 \%$ of the measured value) from calibration. The other item with the flow meter is a tolerance on the set point. The velocity inlet should consider both the low set point with the low bias and the high set point with a high bias. These two conditions describe the inlet velocity high and inlet velocity low cases considered. The other input uncertainties come from other physical conditions like outlet pressure (how much does the outlet pressure condition vary from a standard conditions day?) and the CFD modeling selections (ie Turbulence Model, Wall Function approach, Solver). It is recommended that the input parameters be selected by examining the known uncertainties in the boundary conditions and known (appropriate) models that could simulate the actual physics. This process may be somewhat subjective. The analyst should ask the questions. How well do I know my inputs? What models or codes are applicable to solve this problem? By asking these questions, one could compile a table of input uncertainty parameters. 
Table 11: Parameters for ECS System

\begin{tabular}{|c|c|c|}
\hline Input Variable & Description & Bias \\
\hline Grid & 3 grids considered for each configuration & \\
\hline Inlet Velocity & Boundary Condition low and high & $10 \%$ \\
\hline Outlet Pressure & Boundary Condition low and high & $2 \%$ \\
\hline Turbulence Model & SA, ke-realizable, kwSST & \\
\hline Wall Functions & with and without & \\
\hline $\begin{array}{c}\text { Rough Wall } \\
\text { Function }\end{array}$ & smooth vs. rough & \\
\hline Compressibility & incompressible vs. compressible & \\
\hline Solver & OpenFoam, Fluent, STARCCM+ & $1.36,1.5,2.306 \mathrm{e}-$ \\
\hline & C & 05 \\
\hline
\end{tabular}

The proposed uncertainty calculation is shown in equation 42 and 43 . The process is the same as described in section 2.3. The k-value of 1.746 was used because there are seventeen different CFD cases considered for each configuration. The cases are listed in Table 12. 
Table 12: Cases Solved

Configuration

Parameter

$\begin{array}{lll}0.75 & 3.5 & 5.5\end{array}$

Case \#

Grid

\begin{tabular}{|c|c|c|c|c|c|}
\hline 1 & coarse & 1 & 1 & 1 \\
\hline 2 & med & 2 & 2 & 2 \\
\hline 3 & fine & 3 & 3 & 3 \\
\hline
\end{tabular}

Boundary

Conditions

\begin{tabular}{|l|l|c|c|c|c|}
\hline 4 & & inlet velocity low & 4 & 4 & 4 \\
\hline 5 & & inlet velocity high & 5 & 5 & 5 \\
\hline 6 & & pressure outlet low & 6 & 6 & 6 \\
\hline 7 & & pressure outlet high & 7 & 7 & 7 \\
\hline
\end{tabular}

Turbulence Models

\begin{tabular}{|c|c|c|c|c|c|}
\hline 8 & & SA & 8 & 8 & 8 \\
\hline 9 & & ke-realizable - same as1 & 9 & 9 & 9 \\
\hline 10 & & kwsst & 10 & 10 & 10 \\
\hline
\end{tabular}

\begin{tabular}{|c|c|c|c|c|c|}
\hline 11 & Wall Functions & without wall functions & 11 & 11 & 11 \\
\hline 12 & Surface Roughness & rough wall function & 12 & 12 & 12 \\
\hline & Compressibility & $\begin{array}{c}\text { different openfoam } \\
\text { solver }\end{array}$ & 13 & 13 & 13 \\
\hline
\end{tabular}

Solver

\begin{tabular}{|l|l|c|c|c|c|}
\hline 14 & & fluent & 14 & 14 & 14 \\
\hline 15 & & starccm & 15 & 15 & 15 \\
\hline
\end{tabular}

Fluid Properties

\begin{tabular}{|l|l|c|c|c|c|}
\hline 16 & & nut high & 16 & 16 & 16 \\
\hline 17 & & nut low & 17 & 17 & 17 \\
\hline
\end{tabular}




$$
\begin{aligned}
u_{\text {val }}=1.746 & +\left(\left(\frac{\partial V}{\partial \text { grid }}\right)^{2} B_{\text {grid }}^{2}\right)+\left(\left(\frac{\partial V}{\text { dinletvelocity }}\right)^{2} B_{\text {inletvelocity }}^{2}\right. \\
& +\left(\left(\frac{\partial V}{\text { doutletpressure }}\right)^{2} B_{\text {outletpressure }}^{2}\right. \\
& +\left(\left(\frac{\partial V}{\partial \text { turbulence }}\right)^{2} B_{\text {turbulence }}^{2}\right) \\
& +\left(\frac{\partial V}{\partial \text { wallfunctions }}\right)^{2} B_{\text {wallfunctions }}^{2}+\left(\frac{\partial V}{\partial r o u g h w a l l}\right)^{2} B_{\text {roughwall }}^{2} \\
& +\left(\frac{\partial V}{\partial \text { compressibility }}\right)^{2} B_{\text {compressibility }}^{2}+\left(\frac{\partial V}{\partial s o l v e r}\right)^{2} B_{\text {solver }}^{2} \\
& \left.+\left(\frac{\partial V}{\partial f \text { fluidproperties }}\right)^{2} B_{\text {fluidproperties }}^{2}\right)^{1 / 2} \\
& \quad u_{\text {val }}=1.746 *\left|\frac{1}{2}\left(S_{U}-S_{L}\right)\right|
\end{aligned}
$$

\section{$6.1 \quad \underline{0.75 m \text { Configuration }}$}

The $0.75 \mathrm{~m}$ configuration was analyzed according to the methodology laid out above and a solution iterated on three separate grids and all input variables considered in the uncertainty analysis. The solution is shown in section 6.1.1, the uncertainty in section 6.1.2, the ranking of input parameters in section 6.1.3. Section 6.1.4 is a discussion of the data presented. 


\subsubsection{Solution}

Contour plots of the $0.75 \mathrm{~m}$ configuration are shown in this section along with corresponding line plots. The line plots include all of the models considered in the uncertainty analysis.

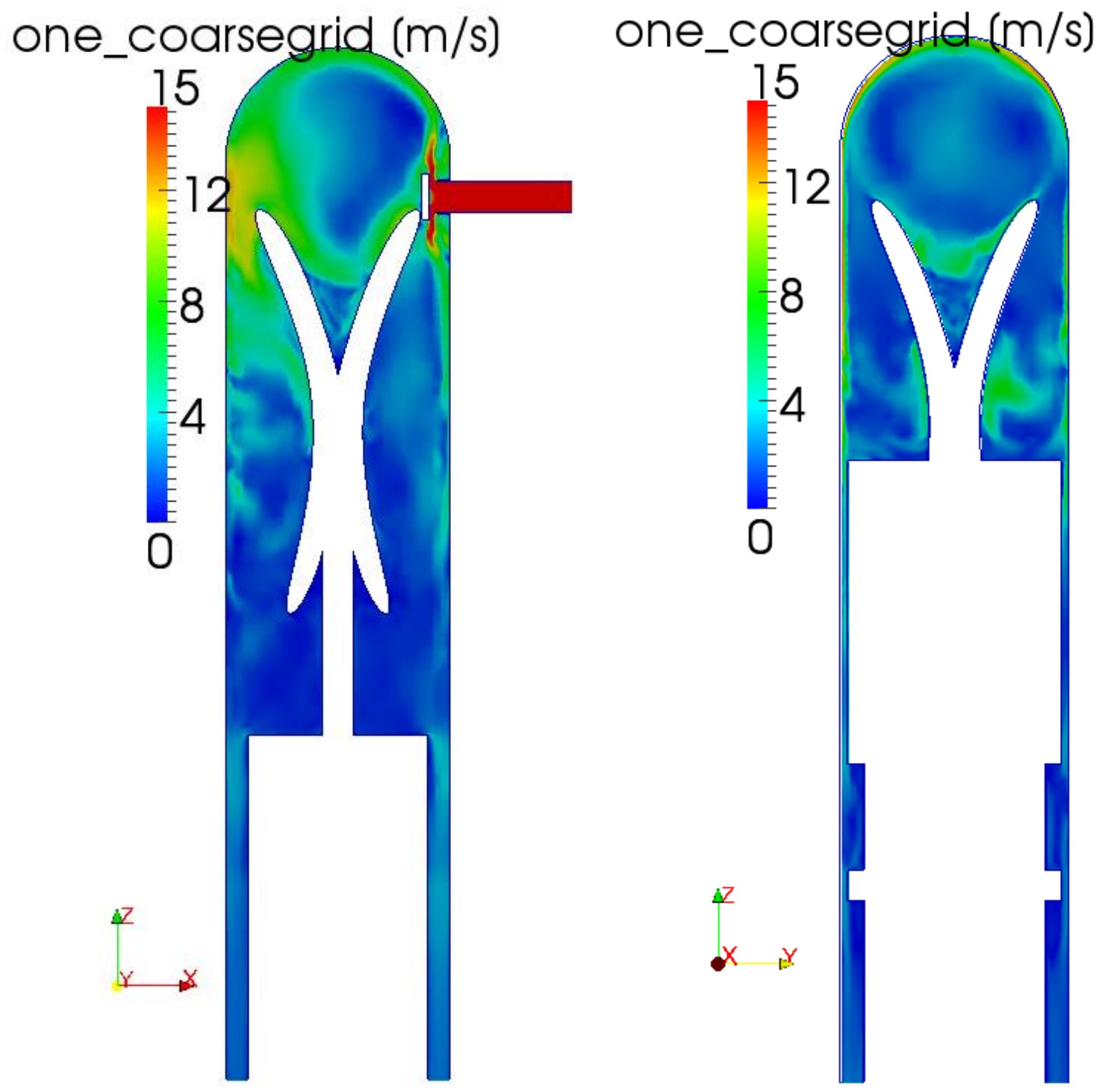

Figure 60: CFD Results for 0.75m Configuration - Contour Plot 


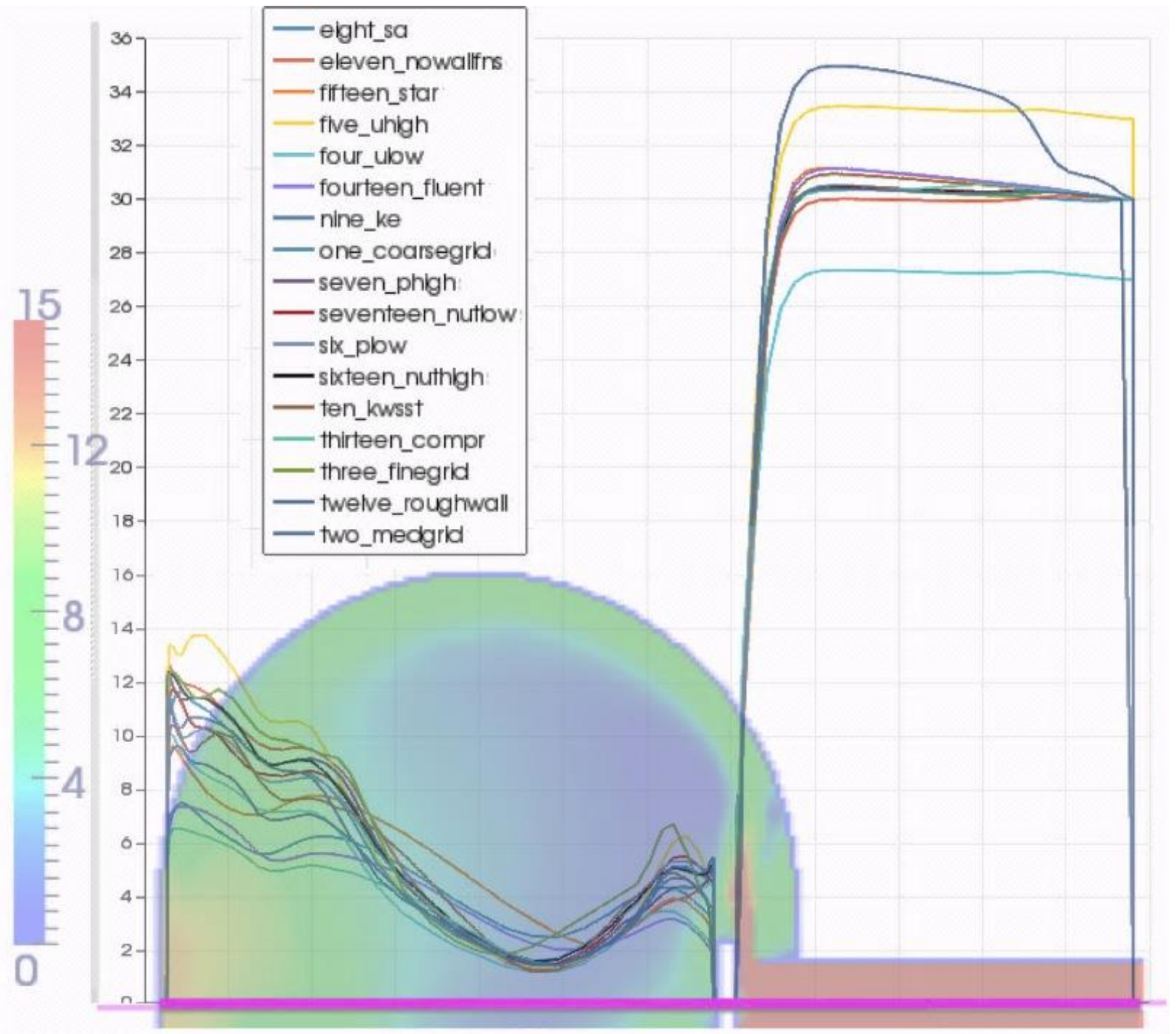

Figure 61: CFD Results for 0.75m Configuration - Line Plot (m/s) 


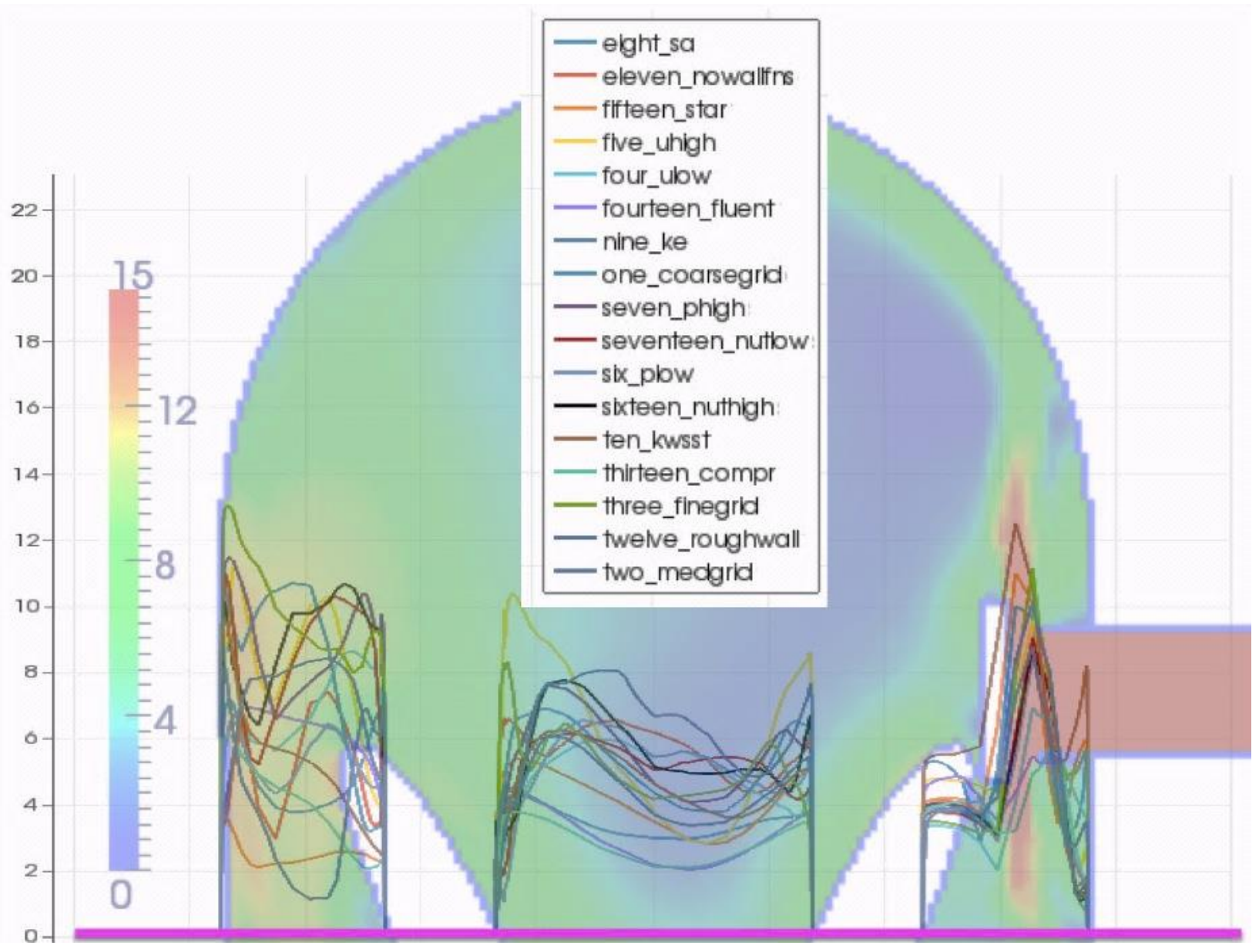

Figure 62: CFD Results for $0.75 \mathrm{~m}$ Configuration - Line Plot (m/s) 


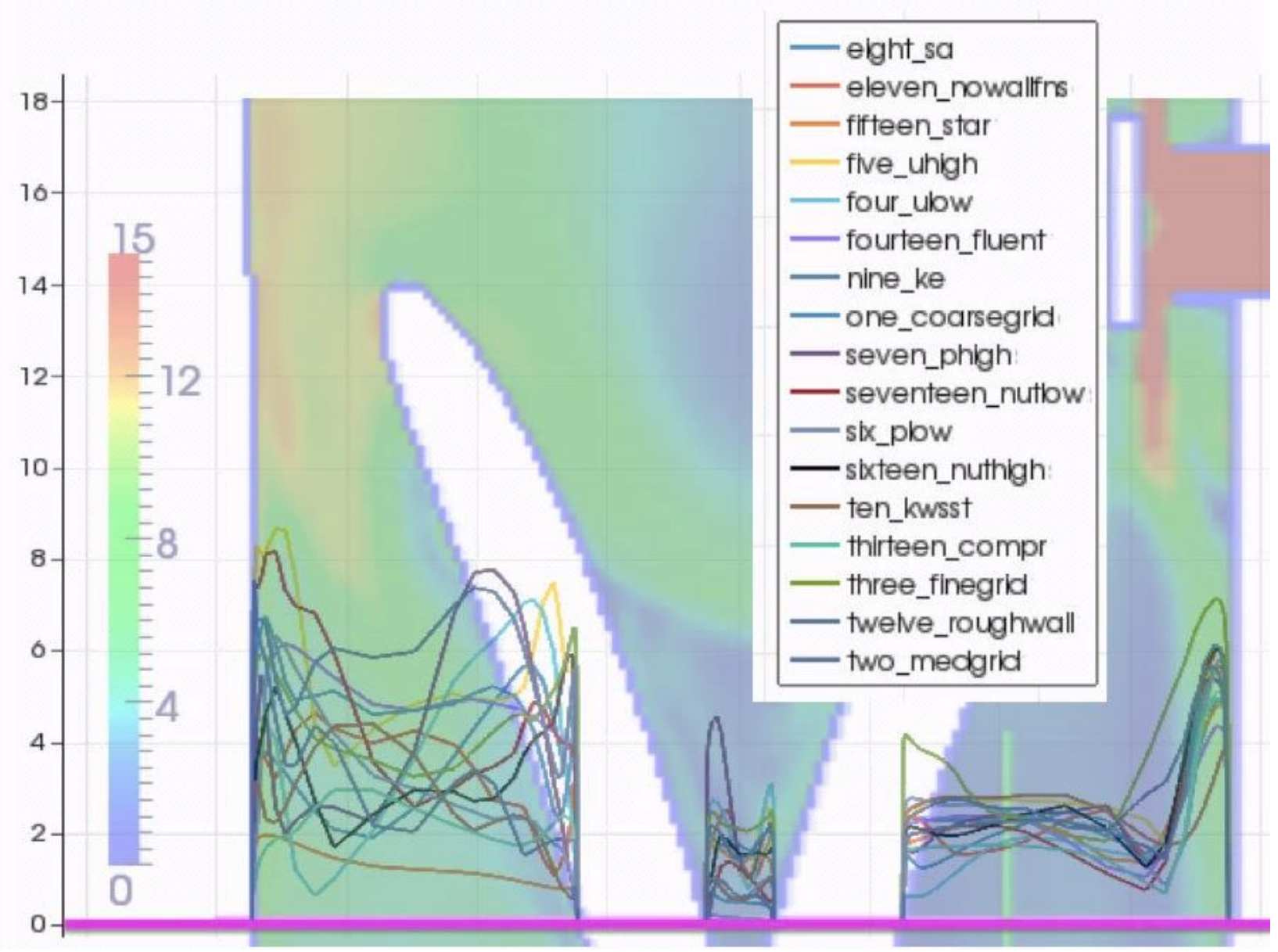

Figure 63: CFD Results for $0.75 \mathrm{~m}$ Configuration - Line Plot (m/s) 


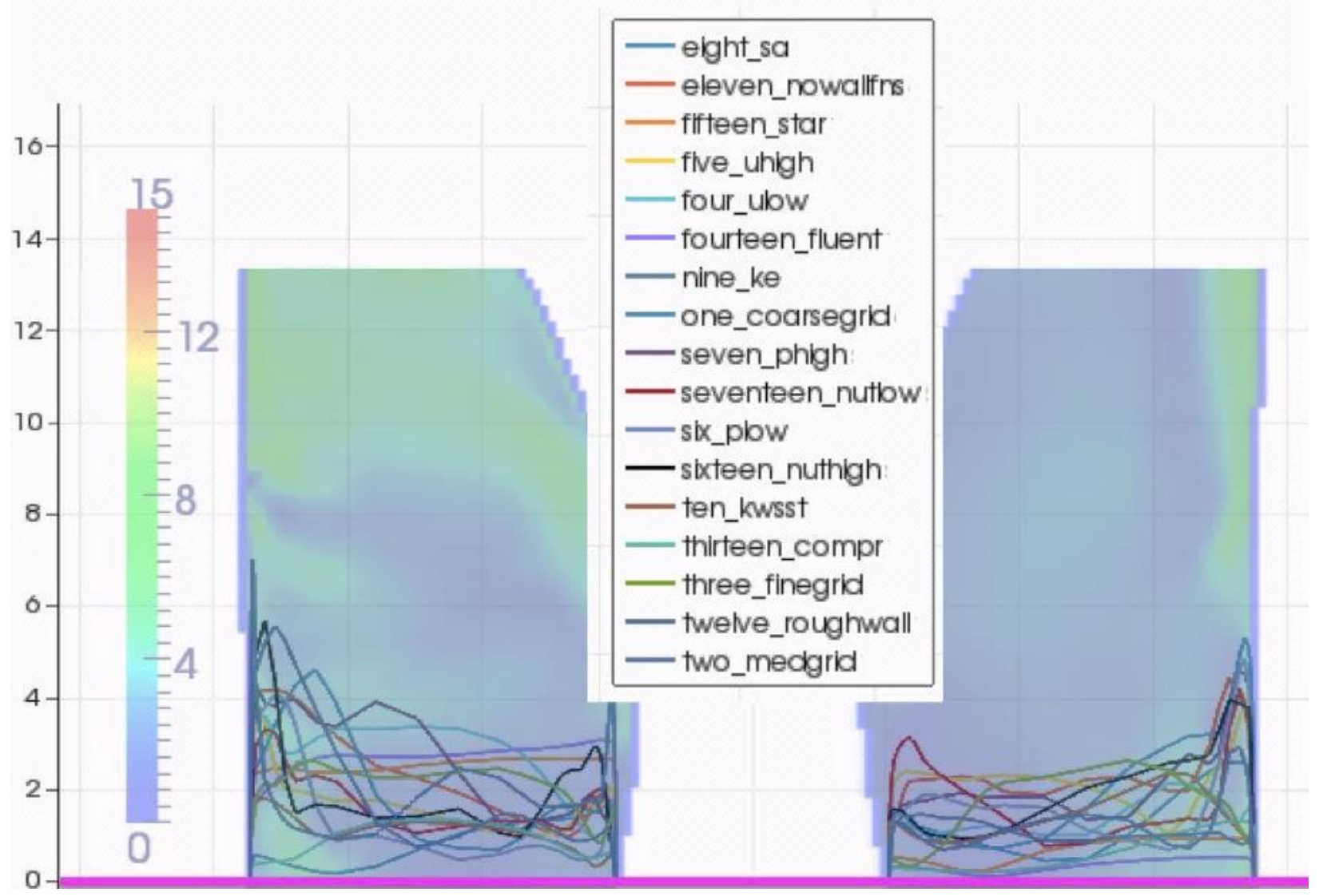

Figure 64: CFD Results for 0.75m Configuration - Line Plot (m/s)

\subsubsection{Uncertainty}

The uncertainty was calculated using equation 29 . The process was to find the minimum, maximum, and middle for each point in the domain and add or subtract the uncertainty calculated from equation 29. Figure 65 plots the results in contour plot with the corresponding uncertainty contour plot. 


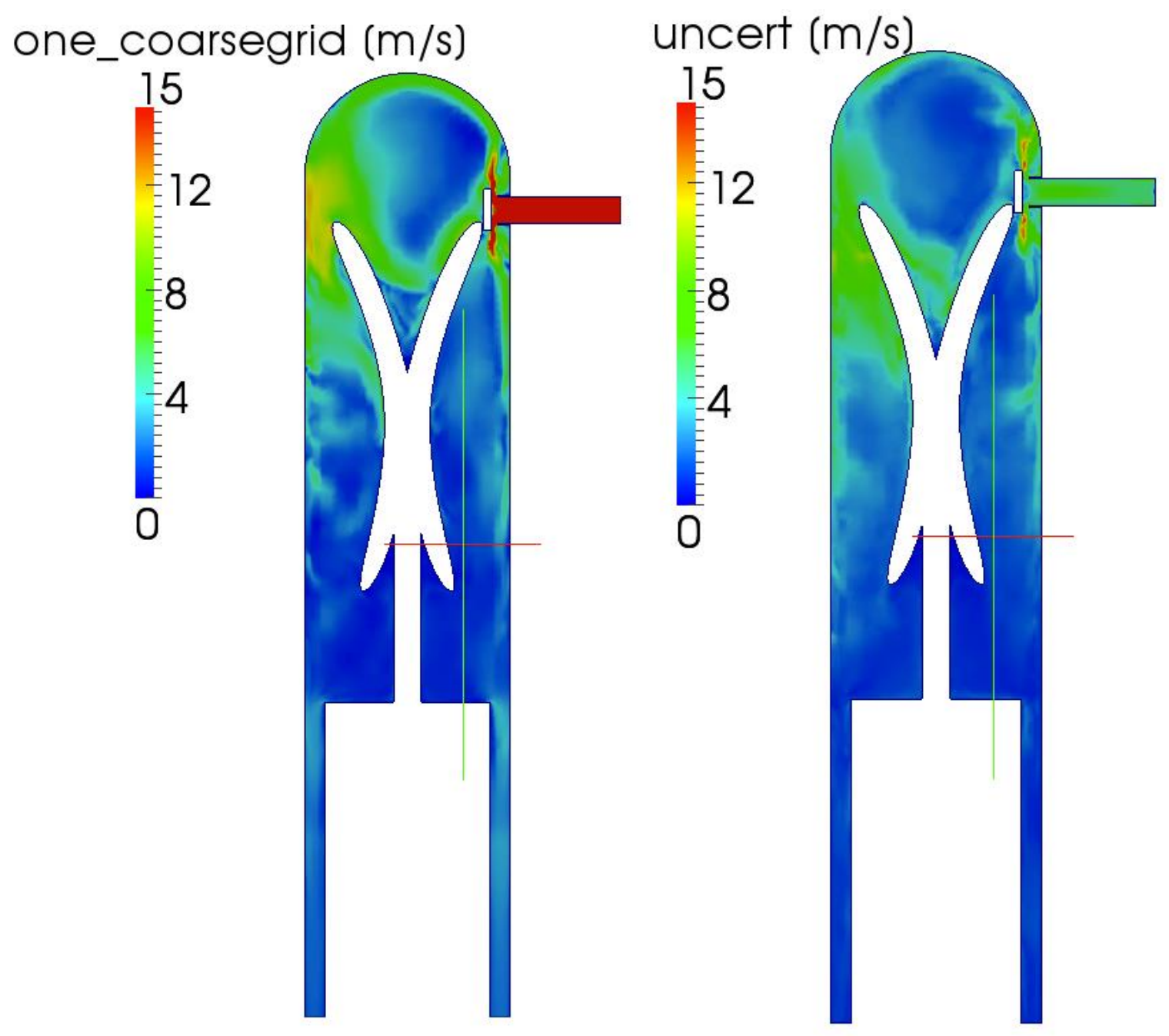

Figure 65: CFD Results and Uncertainty for $0.75 \mathrm{~m}$ Configuration - Contour Plot (m/s)

A more qualitative way to view the results is to plot the uncertainty minimum and maximum for the locations of interest in the domain on a line plot. This is shown in Figure 66 to Figure 69. 


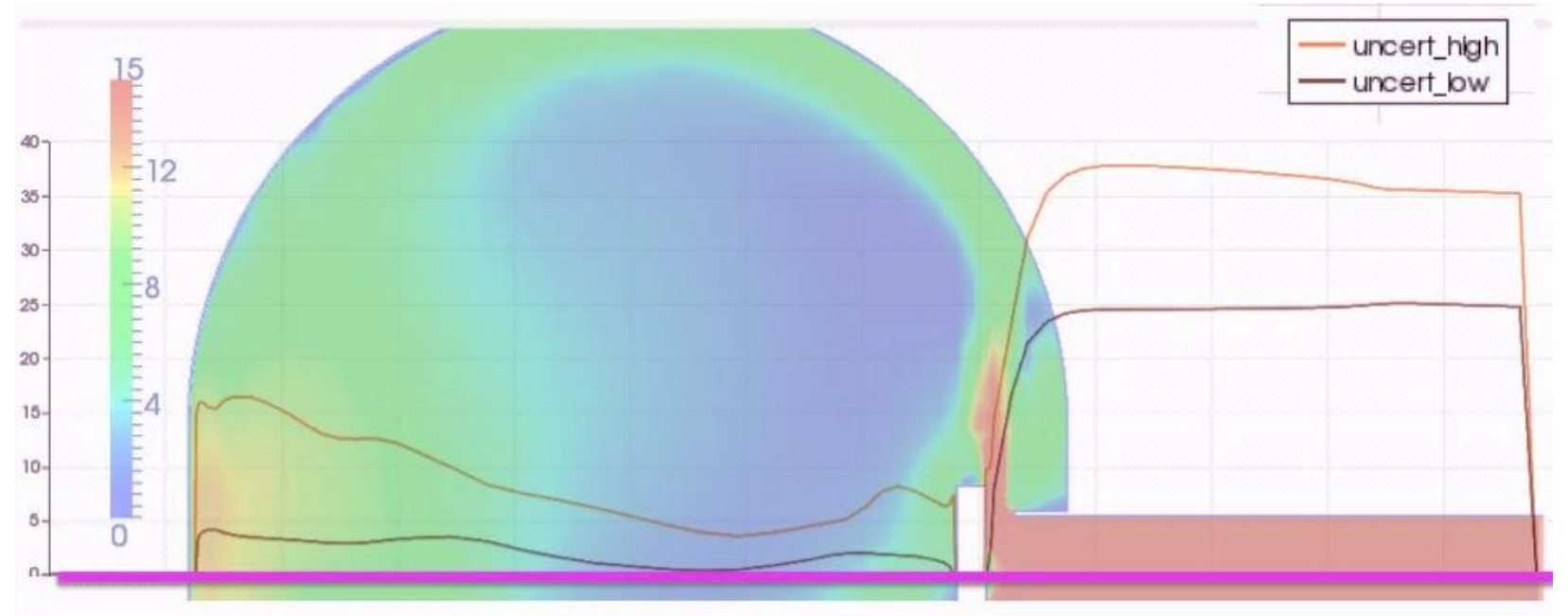

Figure 66: CFD Uncertainty Bounds for $0.75 \mathrm{~m}$ Configuration - Line Plot (m/s)

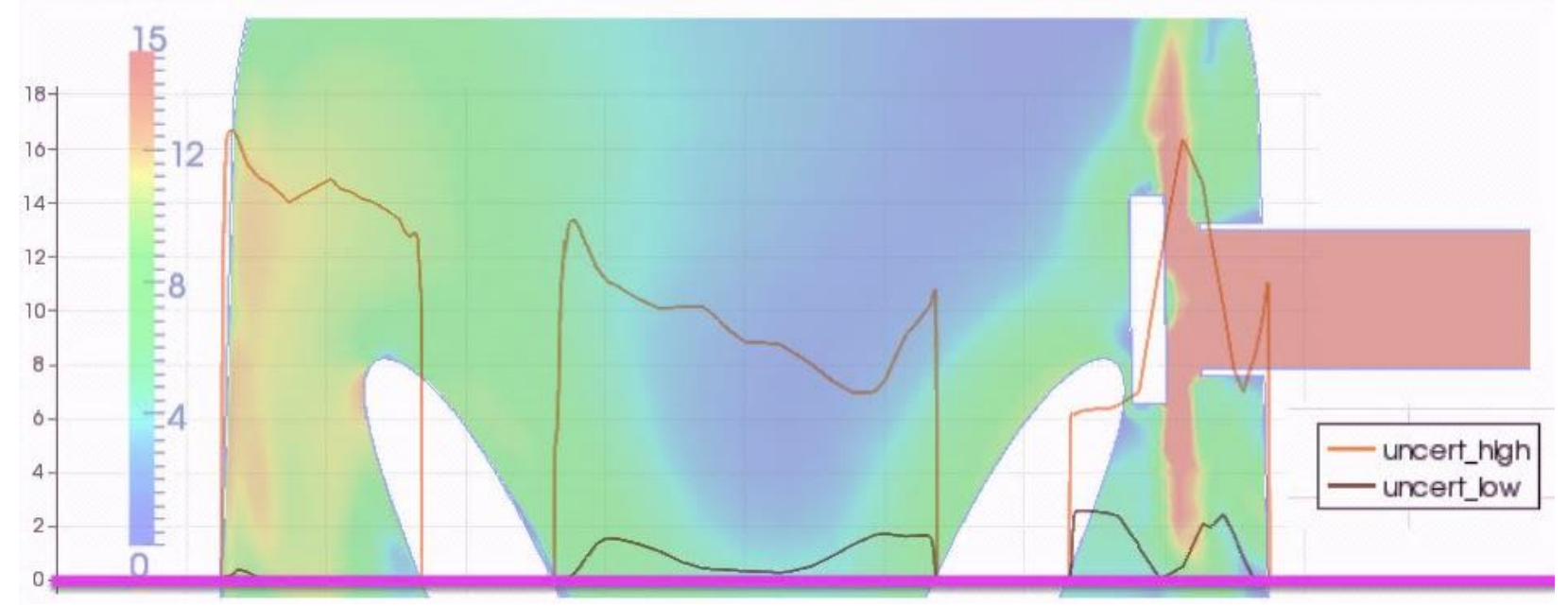

Figure 67: CFD Uncertainty Bounds for $0.75 \mathrm{~m}$ Configuration - Line Plot (m/s) 


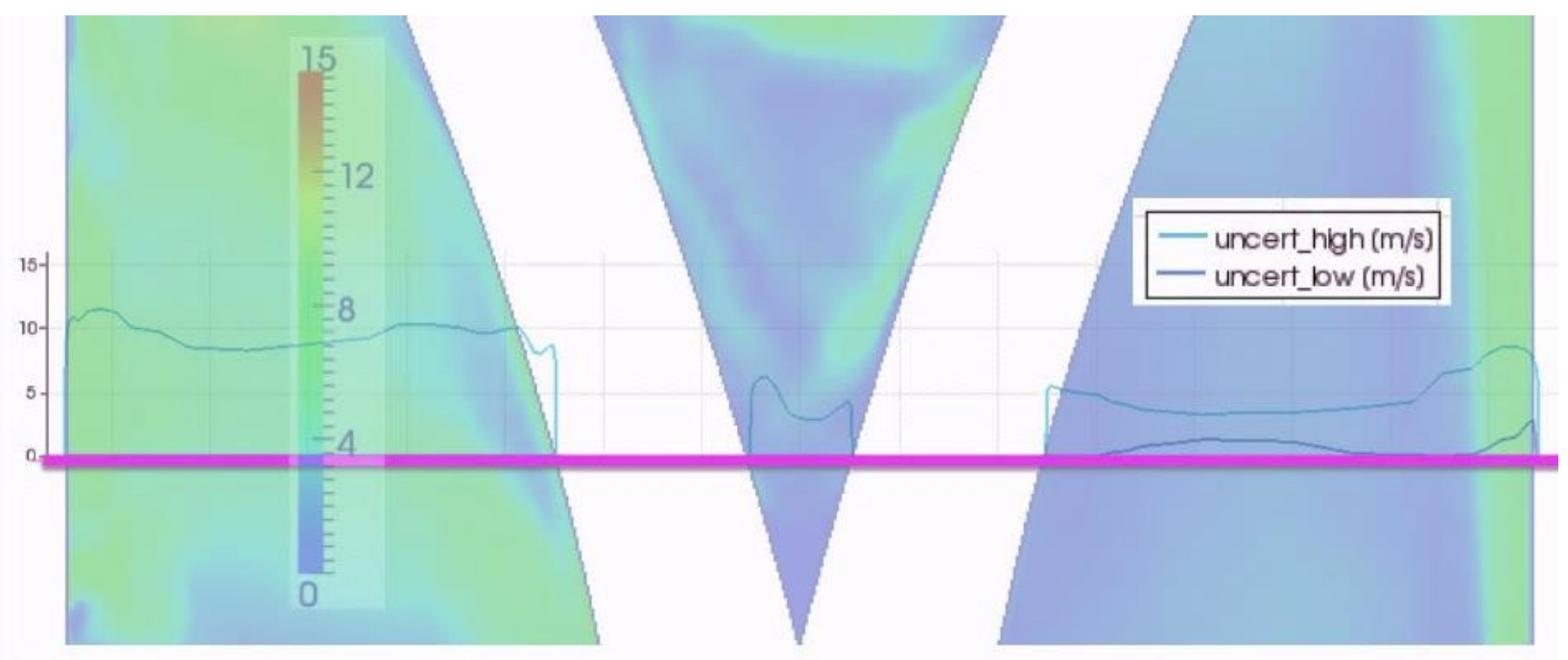

Figure 68: CFD Uncertainty Bounds for $0.75 \mathrm{~m}$ Configuration - Line Plot (m/s)

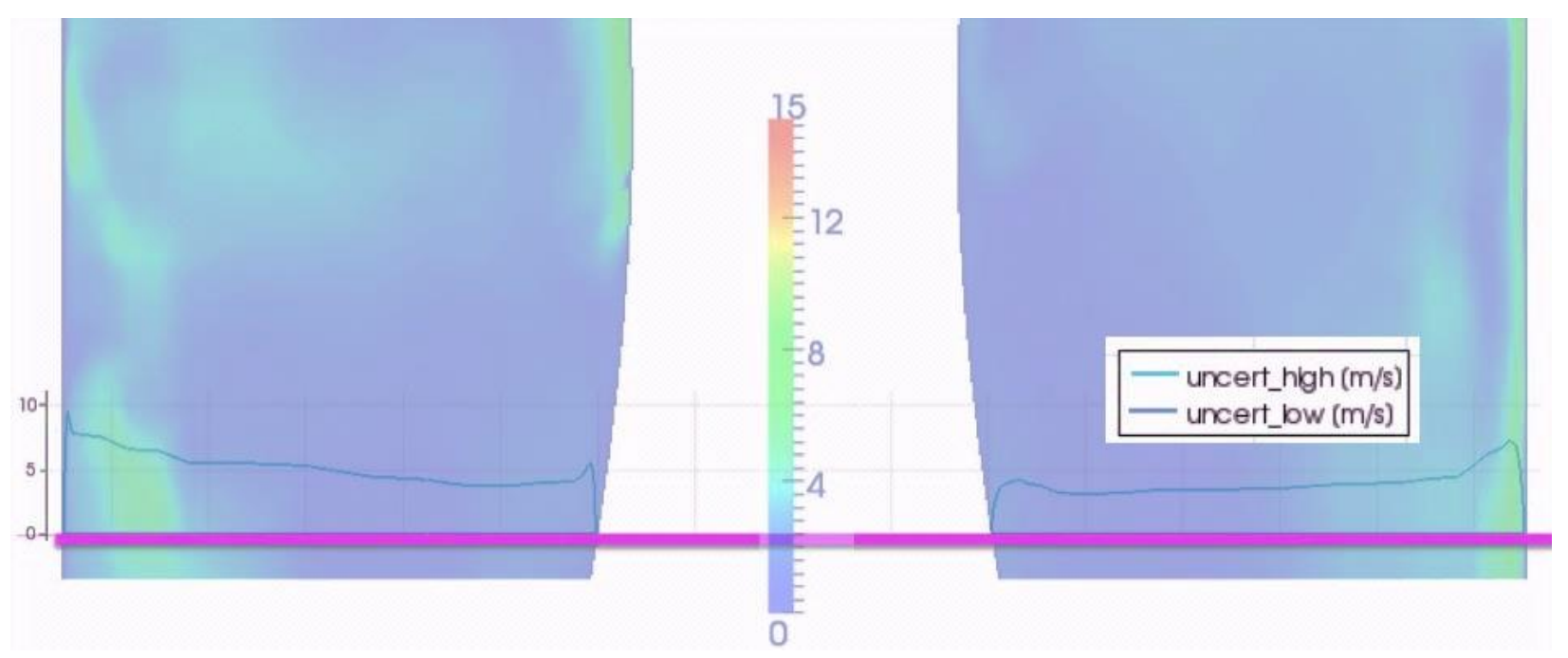

Figure 69: CFD Uncertainty Bounds for $0.75 \mathrm{~m}$ Configuration - Line Plot (m/s) 


\subsubsection{Ranking}

The uncertainty for each of the input variables were ranked by the non-dimensionalizing the difference in the results by the freestream value and ranking from greatest uncertainty to least uncertainty. The results are provided in Table 13. The mean velocity uncertainty is the average of all the cells uncertainty by input variable. The normalized ranking percentage is calculated by adding all the mean non-dimensionalized uncertainties and normalizing each input variable by the sum. The ranking is numbered from 1 to 9 and corresponds to the input variables which have the most uncertainty from greatest to least. This was done to rank the inputs from most uncertain to least uncertain in terms of the velocity magnitude results. 
Table 13: 0.75m Configuration - Non-Dimensional Ranking of Uncertainty

\begin{tabular}{|c|c|c|c|c|c|c|}
\hline $\begin{array}{c}\text { Input } \\
\text { Variable }\end{array}$ & Description & Bias & $\begin{array}{c}\text { Mean } \\
\text { Velocity } \\
\text { Uncertai } \\
\text { nty }(\mathrm{m} / \mathrm{s}) \\
\end{array}$ & $\begin{array}{c}\text { Mean Non- } \\
\text { Dimensionali } \\
\text { zed } \\
\text { Uncertainty }\end{array}$ & $\begin{array}{c}\text { Normali } \\
\text { zed } \\
\text { Ranking } \\
\% \\
\end{array}$ & $\begin{array}{c}\text { Number } \\
\text { ed } \\
\text { Ranking }\end{array}$ \\
\hline Grid & $\begin{array}{c}3 \text { grids } \\
\text { considered }\end{array}$ & & 1.6287 & 0.0543 & 13.40 & 2 \\
\hline $\begin{array}{c}\text { Inlet } \\
\text { Velocity }\end{array}$ & $\begin{array}{l}\text { Boundary } \\
\text { Condition }\end{array}$ & $10 \%$ & 1.3115 & 0.04737 & 11.69 & 5 \\
\hline $\begin{array}{l}\text { Outlet } \\
\text { Pressure }\end{array}$ & $\begin{array}{l}\text { Boundary } \\
\text { Condition }\end{array}$ & $2 \%$ & 1.1478 & 0.0383 & 9.45 & 8 \\
\hline $\begin{array}{c}\text { Turbulence } \\
\text { Model }\end{array}$ & $\begin{array}{l}\text { SA, ke- } \\
\text { realizable, } \\
\text { kwSST }\end{array}$ & & 1.4628 & 0.0488 & 12.04 & 4 \\
\hline $\begin{array}{c}\text { Wall } \\
\text { Functions }\end{array}$ & $\begin{array}{l}\text { with and } \\
\text { without }\end{array}$ & & 0.8286 & 0.0276 & 6.81 & 9 \\
\hline $\begin{array}{c}\text { Rough Wall } \\
\text { Function }\end{array}$ & $\begin{array}{l}\text { smooth vs. } \\
\text { rough }\end{array}$ & & 1.5237 & 0.0508 & 12.53 & 3 \\
\hline $\begin{array}{c}\text { Compressibi } \\
\text { lity }\end{array}$ & $\begin{array}{c}\text { incompressi } \\
\text { ble vs. } \\
\text { compressibl } \\
\text { e }\end{array}$ & & 1.3128 & 0.0438 & 10.81 & 6 \\
\hline Solver & $\begin{array}{c}\text { OpenFoam, } \\
\text { Fluent, } \\
\text { STARCCM } \\
+\end{array}$ & & 1.673 & 0.0558 & 13.77 & 1 \\
\hline $\begin{array}{c}\text { Fluid } \\
\text { Properties }\end{array}$ & $\begin{array}{l}\text { kinematic } \\
\text { viscosity nu } \\
\text { represents } \\
\text { air [0-50- } \\
\text { 100] deg C }\end{array}$ & $\begin{array}{c}1.36,1.5,2.30 \\
6 \mathrm{e}-05\end{array}$ & 1.1536 & 0.0385 & 9.50 & 7 \\
\hline
\end{tabular}

\subsubsection{Discussion}

The variable with the most uncertainty for this configuration was the solver. The same grid and boundary conditions were used in OPENFOAM, STARCCM+, and FLUENT. The other items to note are the uncertainty for each input variable is on the same order of magnitude. It was 
hypothesized there may be only a few input variables of significant importance. No order of magnitude analysis can be completed or conclusions drawn about the significance of each input variable. All of the input variables are significant and contribute to the overall uncertainty calculated.

\section{$6.2 \quad 3.5 \mathrm{~m}$ Configuration}

The $3.5 \mathrm{~m}$ configuration was analyzed according to the methodology laid out above and a solution iterated on three separate grids and all input variables considered in the uncertainty analysis. The solution is shown in section 6.2.1, the uncertainty in section 6.2.2, the ranking of input parameters in section 6.2.3. Section 6.2.4 is a discussion of the data presented.

\subsubsection{Solution}

Contour plots of the $3.5 \mathrm{~m}$ configuration are shown in this section along with corresponding line plots. The line plots include all of the models considered in the uncertainty analysis. 

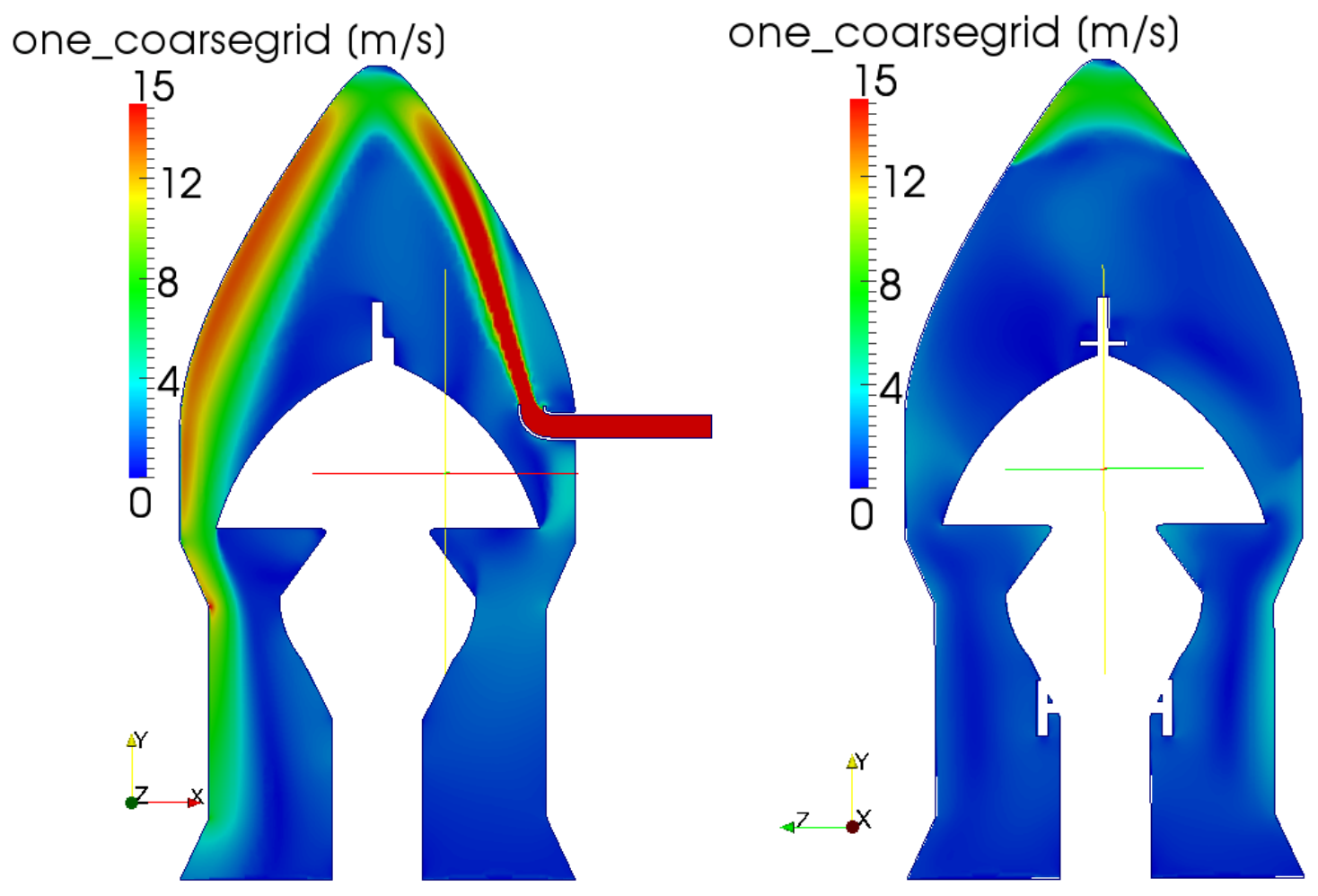

Figure 70: CFD Results for 3.5m Configuration - Contour Plot (m/s) 


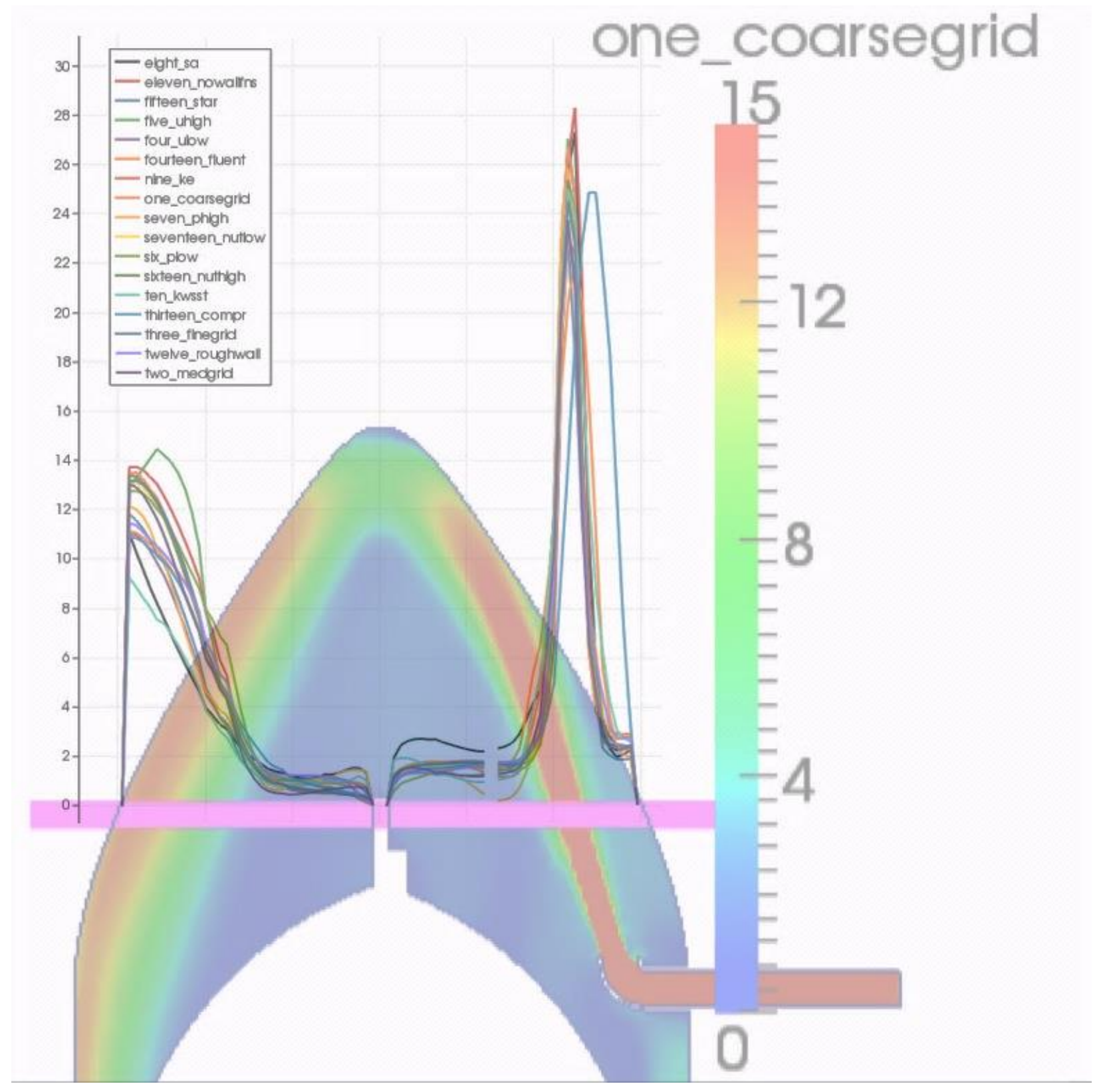

Figure 71: CFD Results for 3.5m Configuration - Line Plot (m/s) 


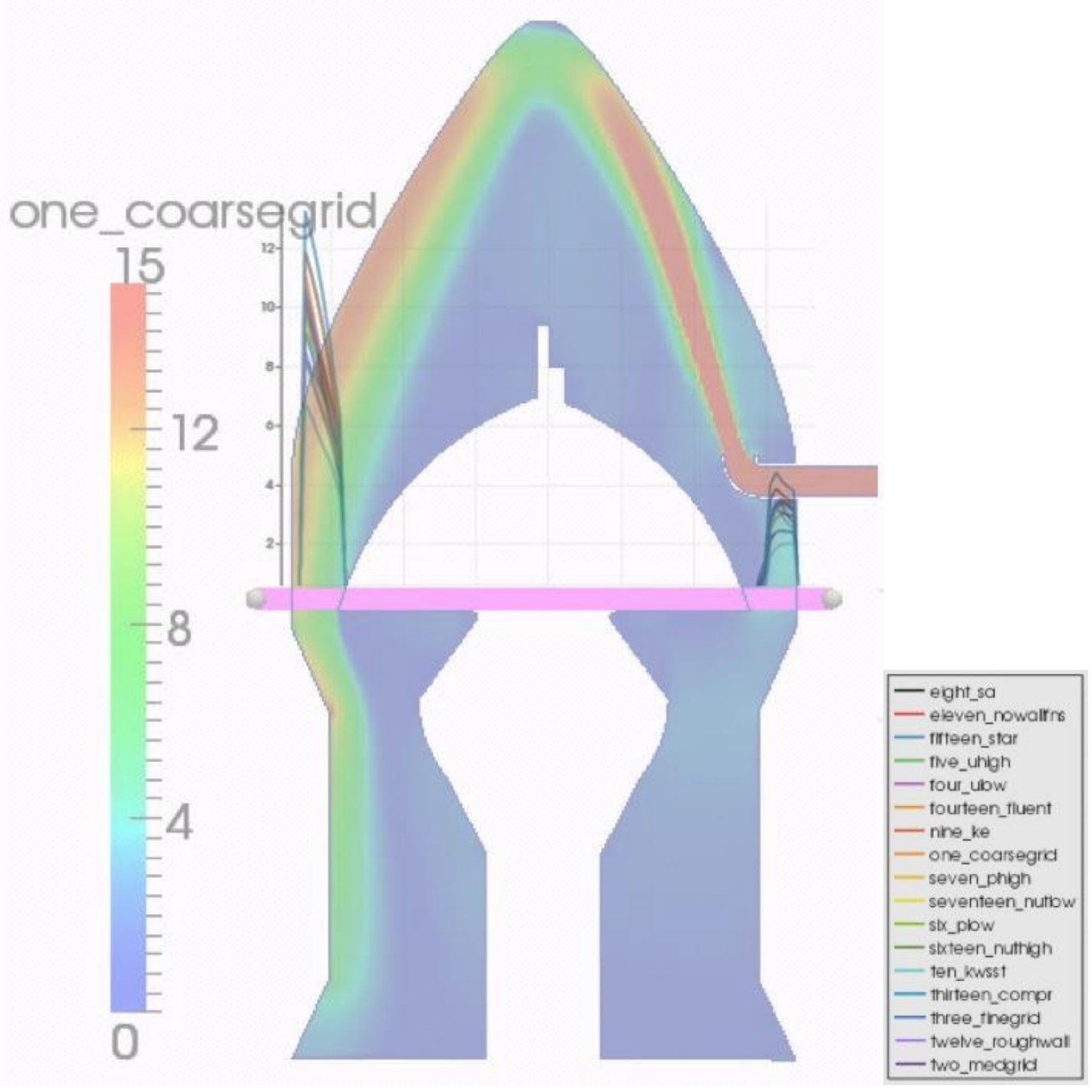

Figure 72: CFD Results for 3.5m Configuration - Line Plot (m/s) 


\subsubsection{Uncertainty}

The uncertainty was calculated using equation 29. The process was to find the minimum, maximum, and middle for each point in the domain and add or subtract the uncertainty calculated from equation 29. Figure 73 plots the results in contour plot with the corresponding uncertainty contour plot.
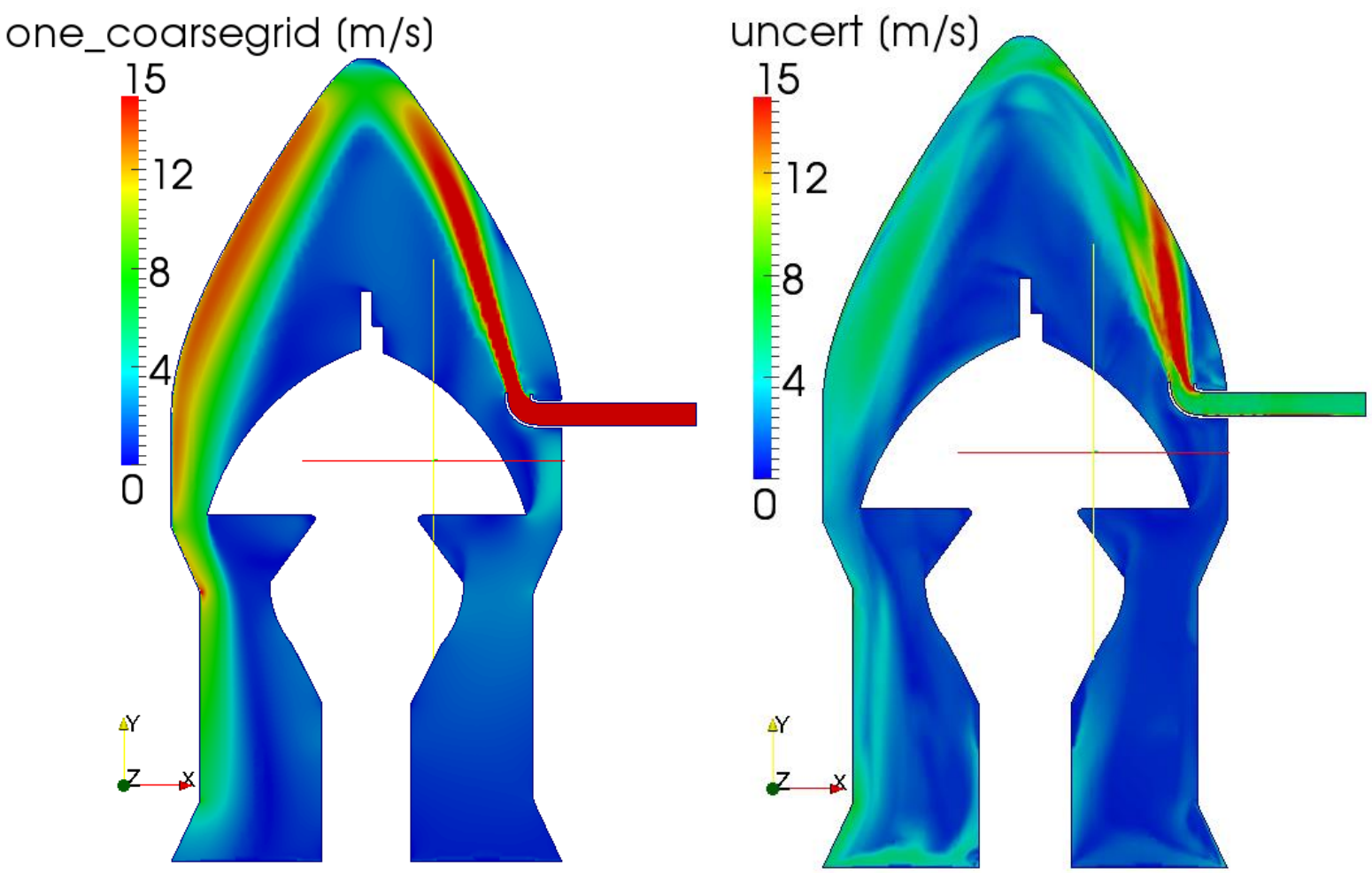

Figure 73: CFD Results and Uncertainty for 3.5m Configuration - Contour Plot (m/s) 


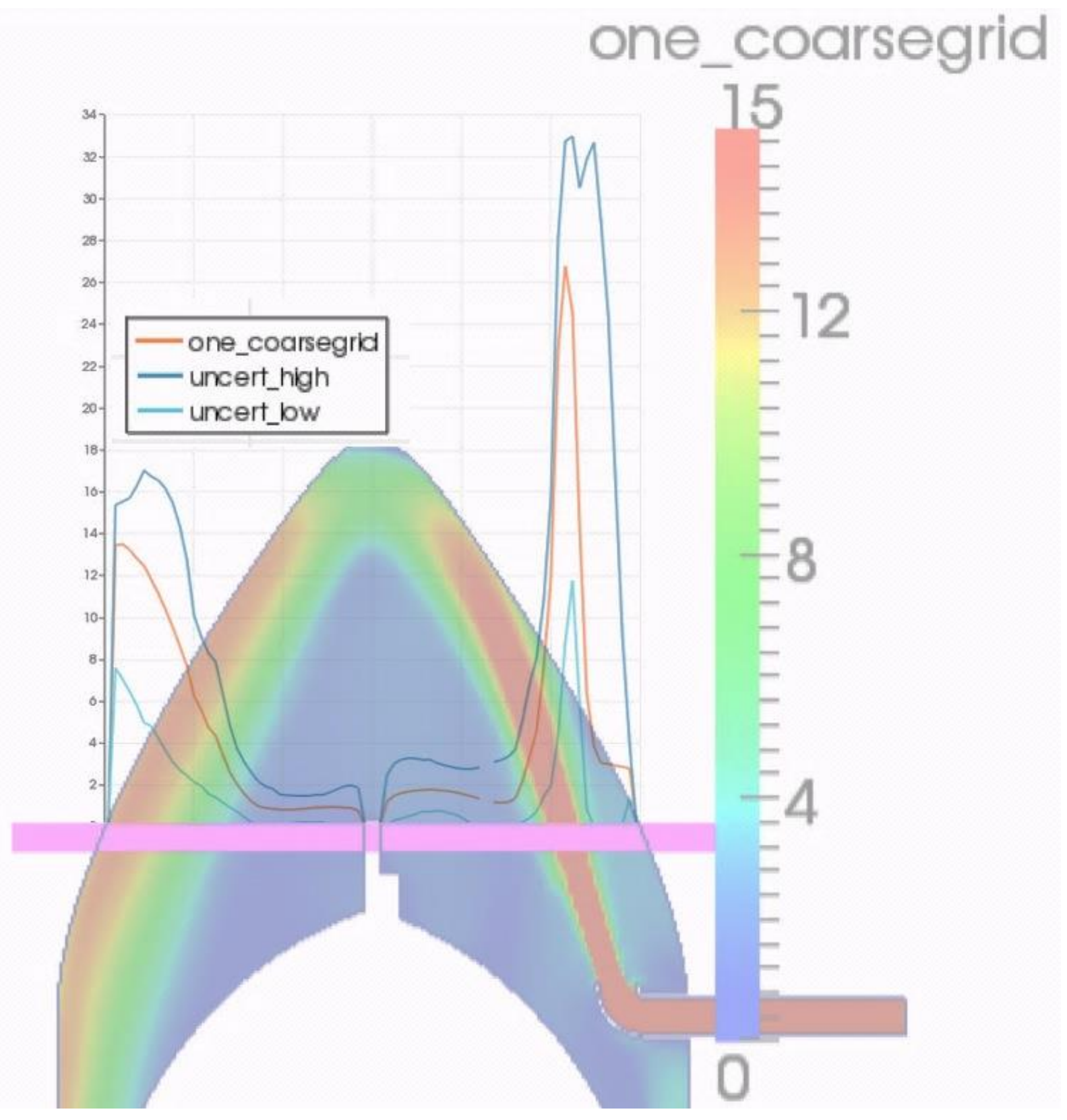

Figure 74: CFD Uncertainty Bounds for 3.5m Configuration - Line Plot (m/s) 


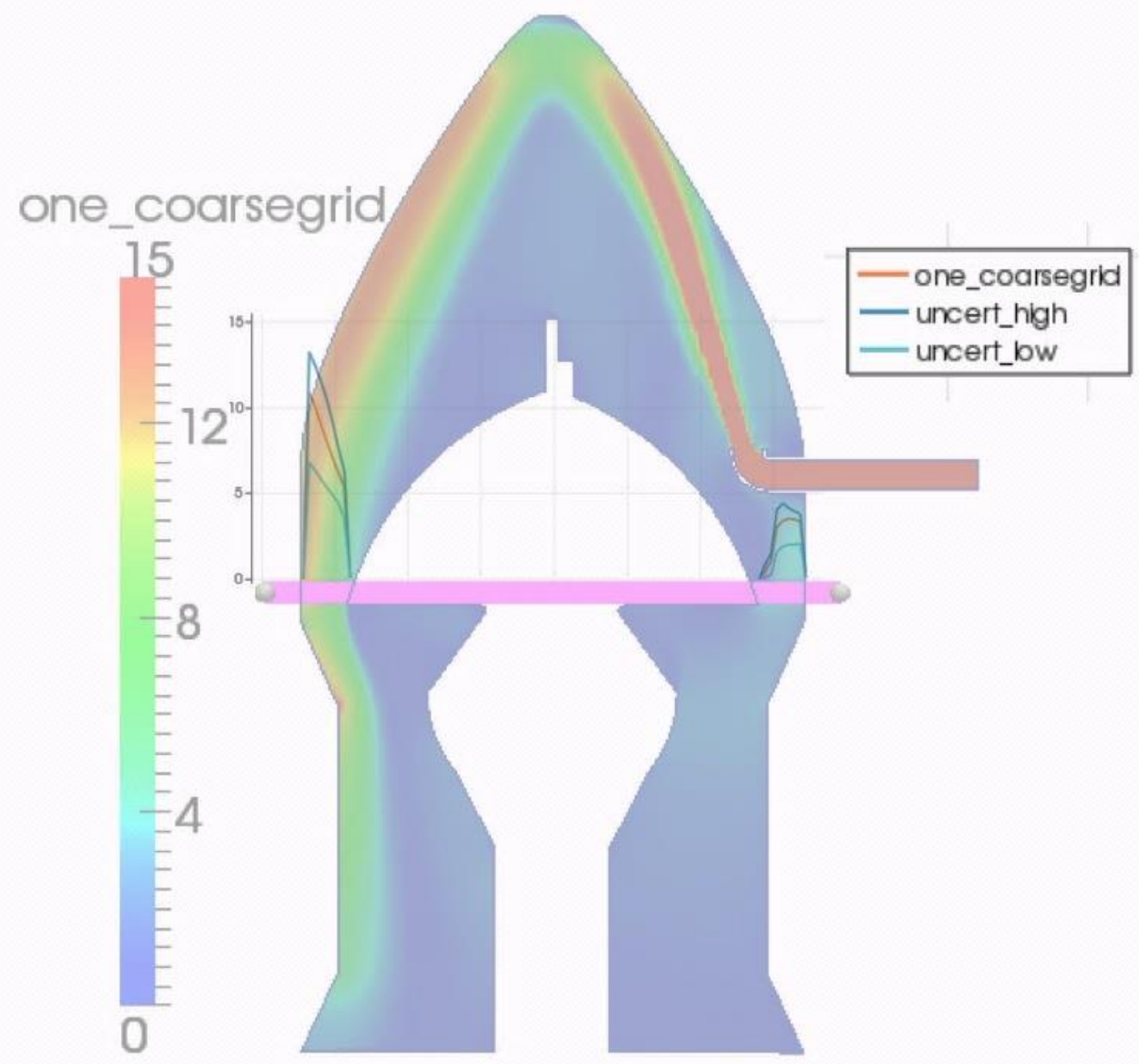

Figure 75: CFD Uncertainty Bounds for 3.5m Configuration - Line Plot (m/s) 


\subsubsection{Ranking}

The uncertainty for each of the input variables were ranked by the non-dimensionalizing the difference in the results by the freestream value and ranking from greatest uncertainty to least uncertainty. The results are provided in Table 14 .

Table 14: 3.5m Configuration - Non-Dimensional Ranking of Uncertainty

\begin{tabular}{|c|c|c|c|c|c|c|}
\hline $\begin{array}{c}\text { Input } \\
\text { Variable } \\
\end{array}$ & Description & Bias & $\begin{array}{c}\text { Mean Velocity } \\
\text { Uncertainty } \\
(\mathbf{m} / \mathbf{s})\end{array}$ & $\begin{array}{c}\text { Mean Non- } \\
\text { Dimensionali } \\
\text { zed } \\
\text { Uncertainty }\end{array}$ & $\begin{array}{c}\begin{array}{c}\text { Normaliz } \\
\text { ed } \\
\text { Ranking } \\
\%\end{array} \\
\end{array}$ & $\begin{array}{c}\text { Numbe } \\
\text { red } \\
\text { Rankin } \\
\text { g } \\
\end{array}$ \\
\hline Grid & $\begin{array}{c}3 \text { grids } \\
\text { considered }\end{array}$ & & 0.6829 & 0.0228 & 8.28 & 7 \\
\hline $\begin{array}{c}\text { Inlet } \\
\text { Velocity }\end{array}$ & $\begin{array}{l}\text { Boundary } \\
\text { Condition }\end{array}$ & $10 \%$ & 0.7919 & 0.0264 & 9.59 & 6 \\
\hline $\begin{array}{c}\text { Outlet } \\
\text { Pressure }\end{array}$ & $\begin{array}{l}\text { Boundary } \\
\text { Condition } \\
\end{array}$ & $2 \%$ & 1.4606 & 0.0487 & 17.70 & 1 \\
\hline $\begin{array}{l}\text { Turbulen } \\
\text { ce Model }\end{array}$ & $\begin{array}{l}\text { SA, ke- } \\
\text { realizable, } \\
\text { kwSST }\end{array}$ & & 1.3487 & 0.045 & 16.35 & 2 \\
\hline $\begin{array}{c}\text { Wall } \\
\text { Function } \\
\mathrm{s} \\
\end{array}$ & $\begin{array}{l}\text { with and } \\
\text { without }\end{array}$ & & 0.6139 & 0.0205 & 7.45 & 9 \\
\hline $\begin{array}{c}\text { Rough } \\
\text { Wall } \\
\text { Function } \\
\end{array}$ & $\begin{array}{l}\text { smooth vs. } \\
\text { rough }\end{array}$ & & 1.0531 & 0.0351 & 12.75 & 3 \\
\hline $\begin{array}{c}\text { Compres } \\
\text { sibility }\end{array}$ & $\begin{array}{l}\text { incompressi } \\
\text { ble vs. } \\
\text { compressibl } \\
\text { e }\end{array}$ & & 0.8252 & 0.0275 & 9.99 & 5 \\
\hline Solver & $\begin{array}{c}\text { OpenFoam, } \\
\text { Fluent, } \\
\text { STARCCM } \\
+ \\
\end{array}$ & & 0.841 & 0.028 & 10.17 & 4 \\
\hline $\begin{array}{c}\text { Fluid } \\
\text { Propertie } \\
\text { s } \\
\end{array}$ & $\begin{array}{c}\text { kinematic } \\
\text { viscosity nu } \\
\text { represents } \\
\text { air [0-50- } \\
100] \text { deg C }\end{array}$ & $\begin{array}{c}1.36,1.5 \\
2.306 \mathrm{e}- \\
05\end{array}$ & 0.6345 & 0.0212 & 7.70 & 8 \\
\hline
\end{tabular}




\subsubsection{Discussion}

The ranking analysis of the $3.5 \mathrm{~m}$ configuration shows a different trend of input variables as being most significant to least significant when compared to the $0.75 \mathrm{~m}$ configuration. The most significant for this configuration was the outlet pressure boundary condition, followed by turbulence model, wall functions, and solver. There is not a significant difference in the uncertainty for the different input variables. Each variable is of the same order of magnitude and cannot be regarded as insignificant to the overall uncertainty calculation.

\section{$6.3 \quad \underline{5.5 m \text { Configuration }}$}

The $5.5 \mathrm{~m}$ configuration was analyzed according to the methodology laid out above and a solution iterated on three separate grids and all input variables considered in the uncertainty analysis. The solution is shown in section 6.3.1, the uncertainty in section 6.3.2, the ranking of input parameters in section 6.3.3. Section 6.3.4 is a discussion of the data presented.

\subsubsection{Solution}

Contour plots of the $5.5 \mathrm{~m}$ configuration are shown in this section along with corresponding line plots. The line plots include all of the models considered in the uncertainty analysis. 


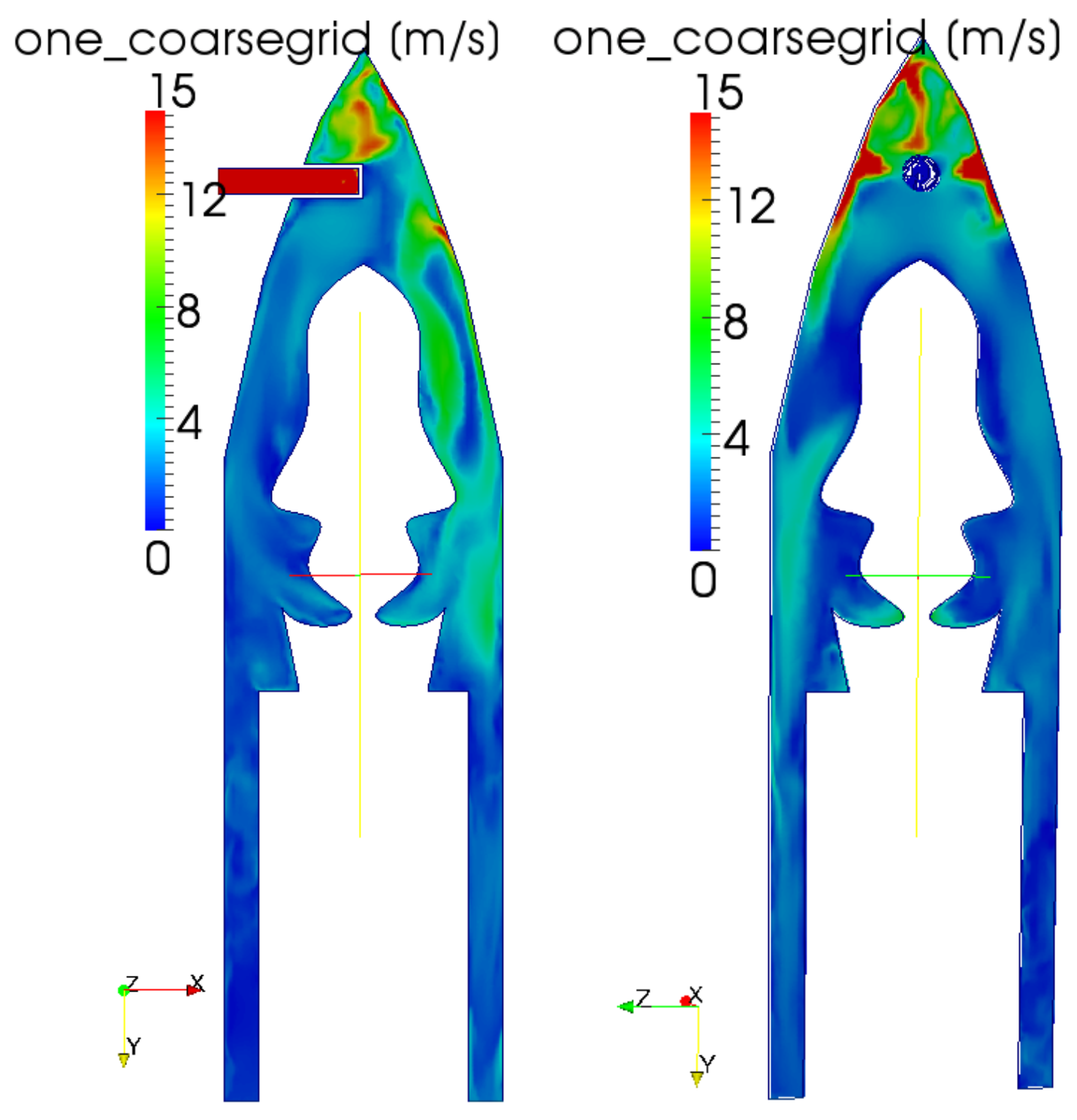

Figure 76: CFD Results for 5.5m Configuration - Contour Plot (m/s) 


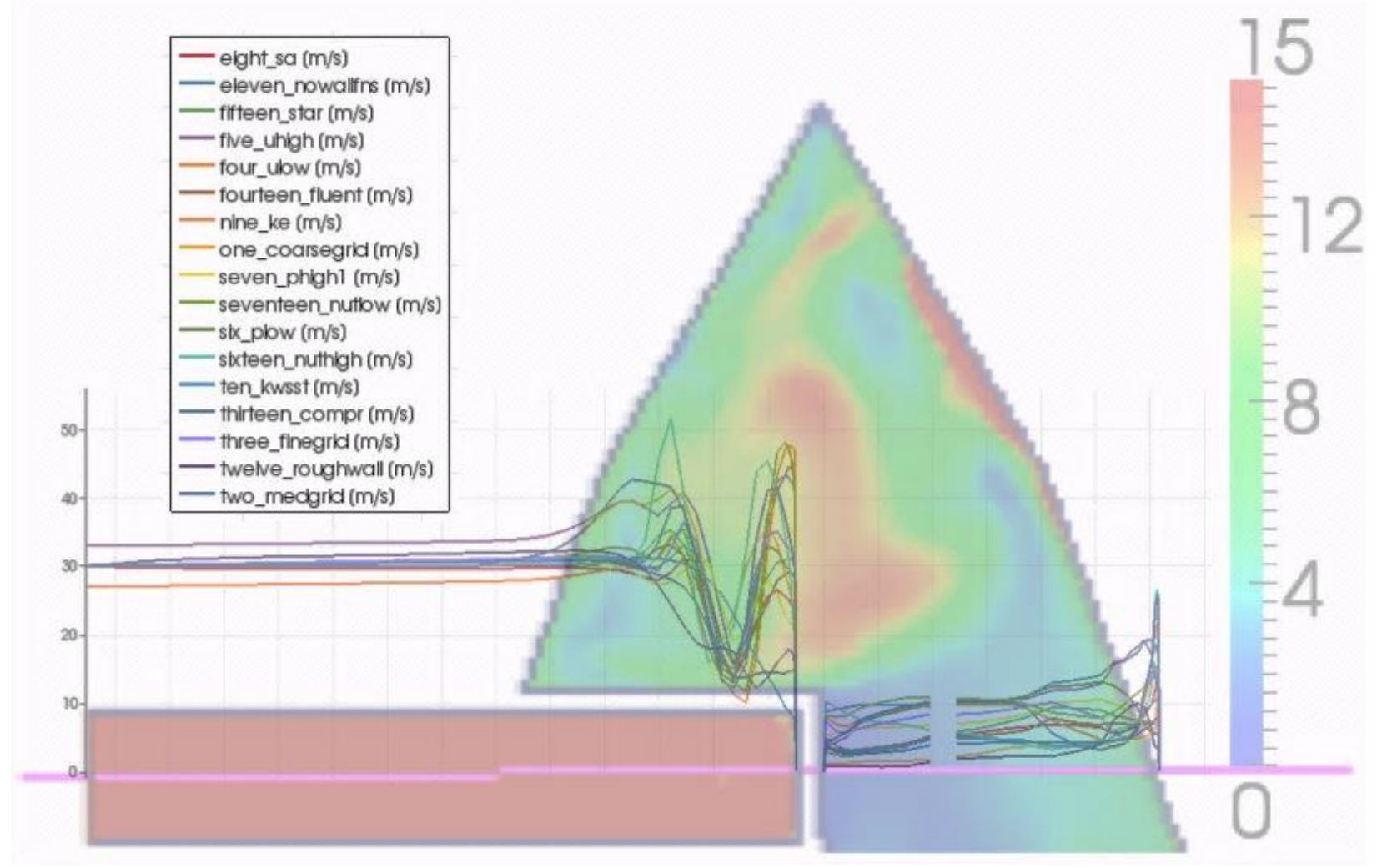

Figure 77: CFD Results for 5.5m Configuration - Line Plot (m/s)

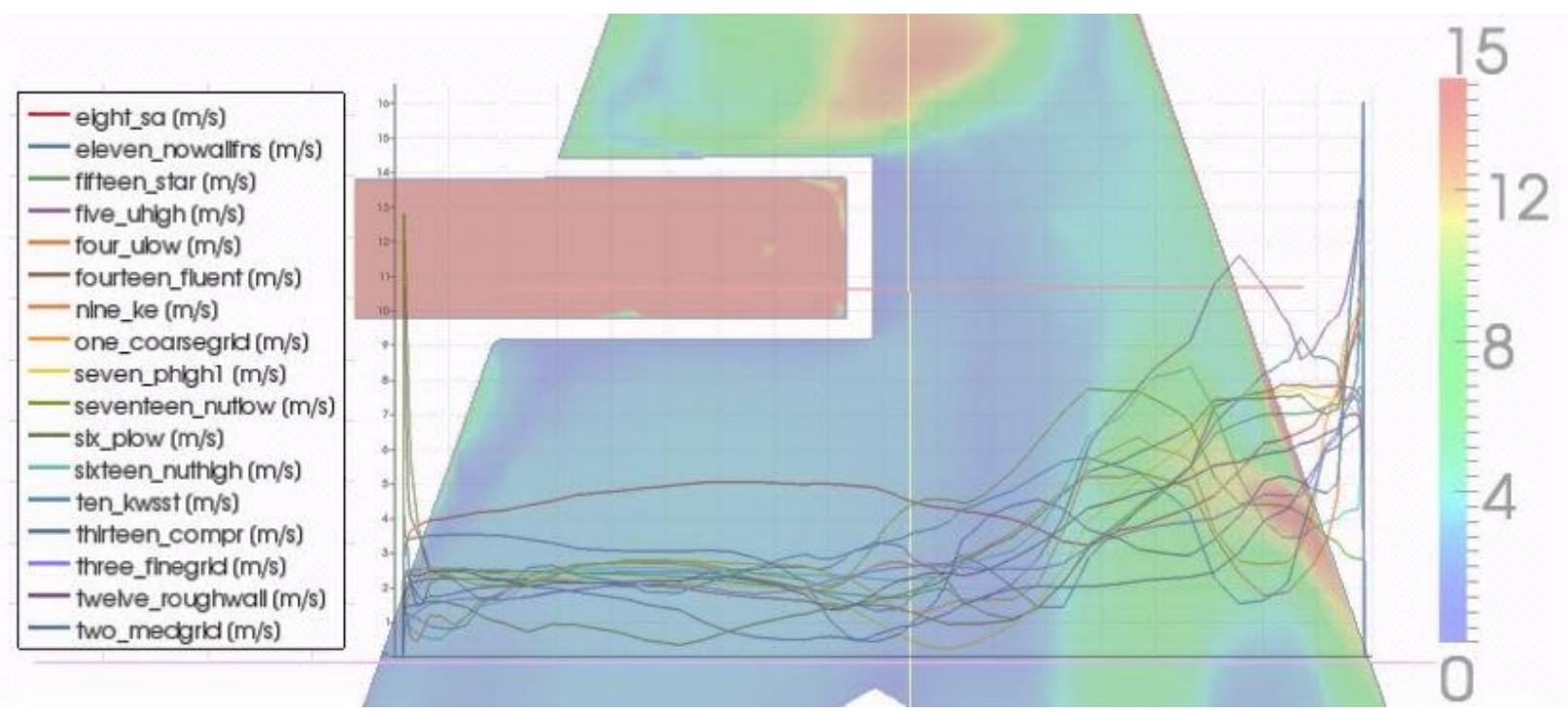

Figure 78: CFD Results for 5.5m Configuration - Line Plot (m/s) 


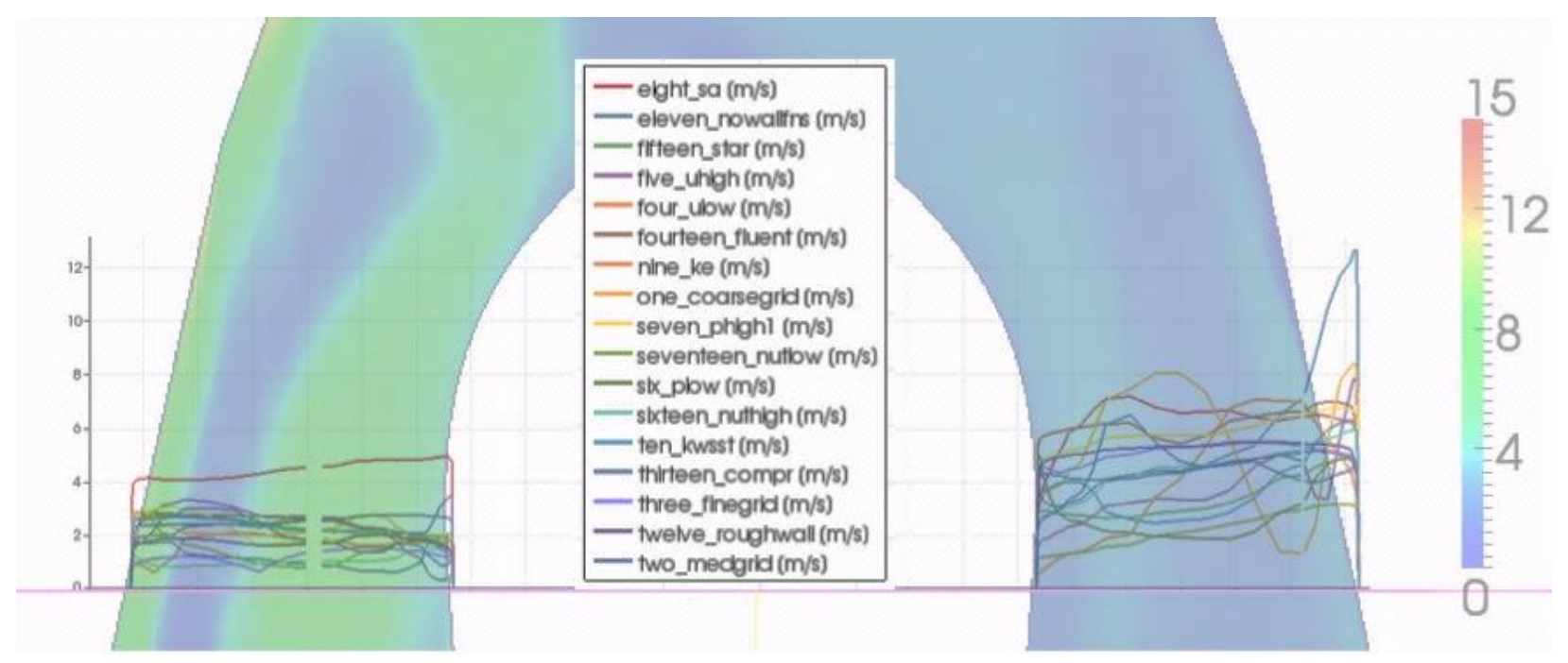

Figure 79: CFD Results for 5.5m Configuration - Line Plot (m/s)

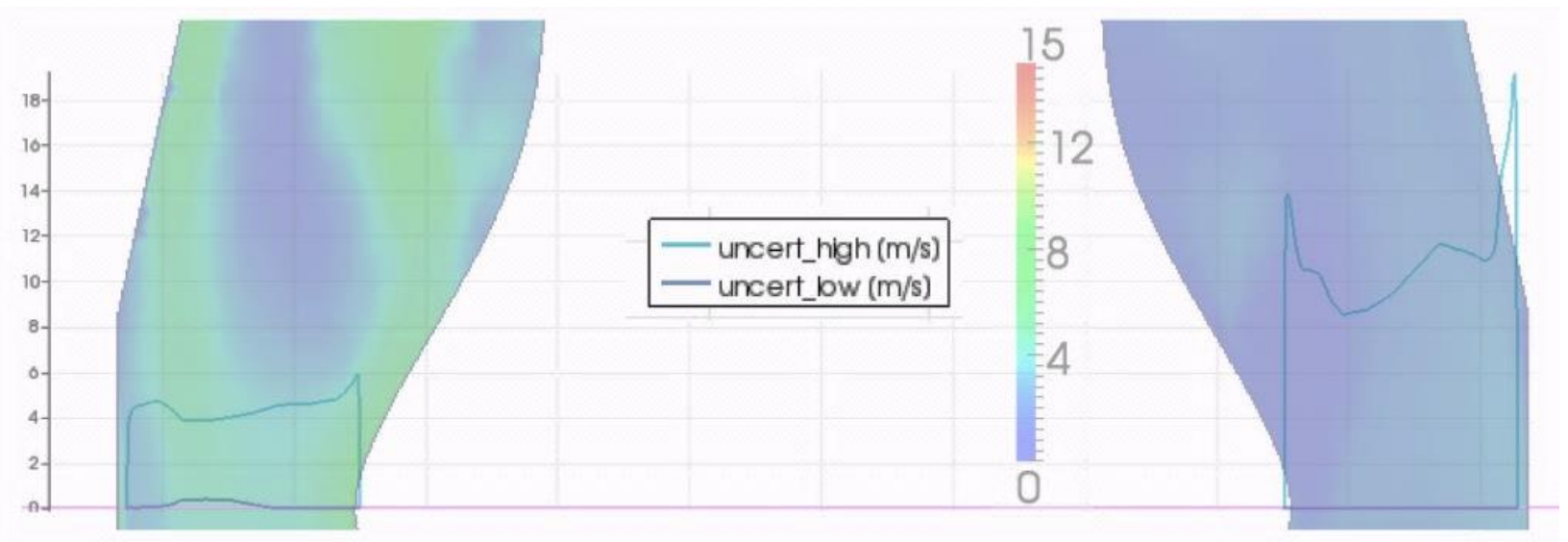

Figure 80: CFD Results for 5.5m Configuration - Line Plot (m/s)

\subsubsection{Uncertainty}

The uncertainty was calculated using equation 29 . The process was to find the minimum, maximum, and middle for each point in the domain and add or subtract the uncertainty calculated 
from equation 29. Figure 81 plots the results in contour plot with the corresponding uncertainty contour plot.

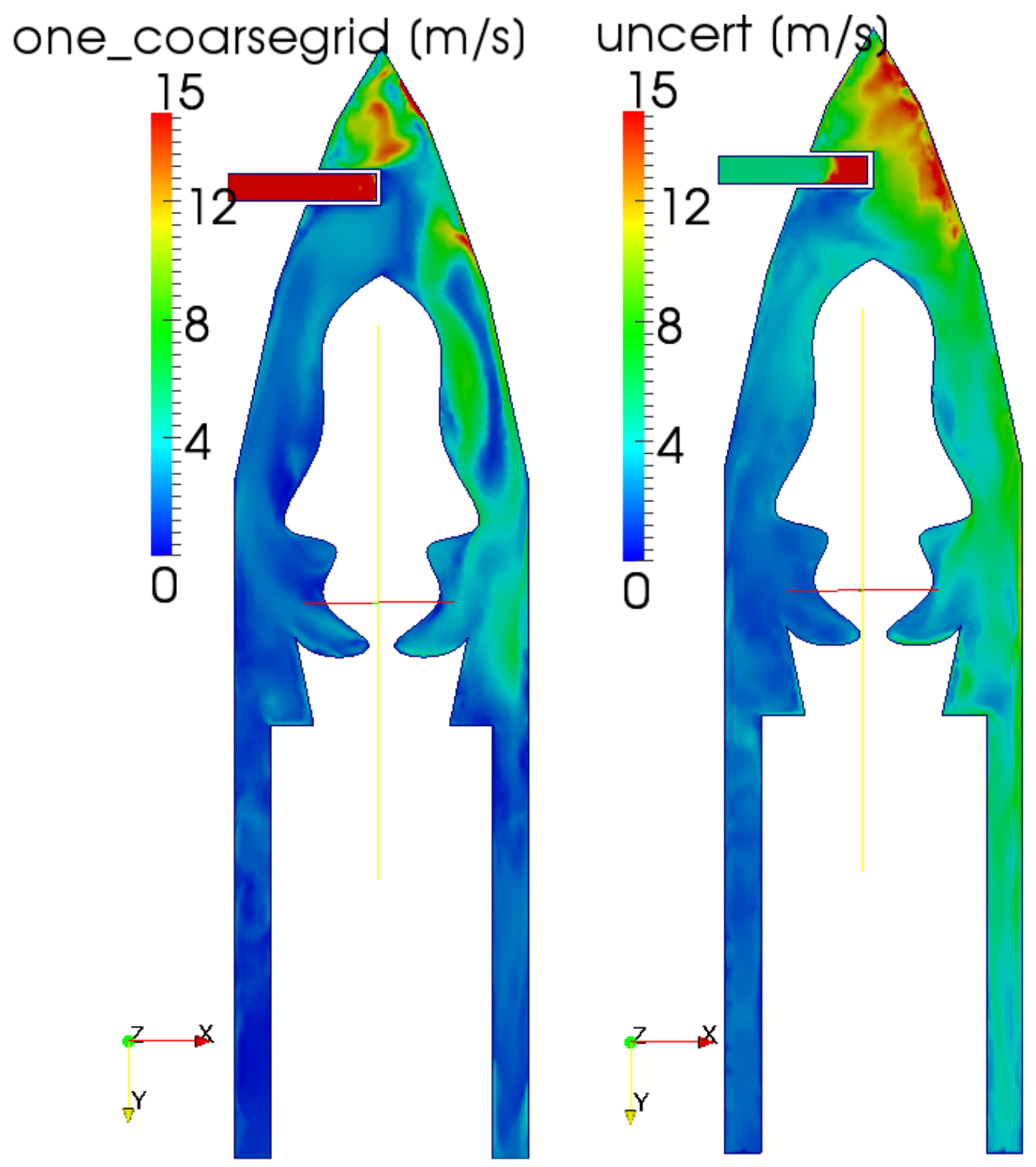

Figure 81: CFD Results and Uncertainty for 5.5m Configuration - Contour Plot (m/s) 
A more qualitative way to view the results is to plot the uncertainty minimum and maximum for the locations of interest in the domain on a line plot. This is shown in Figure 82 to Figure 85.

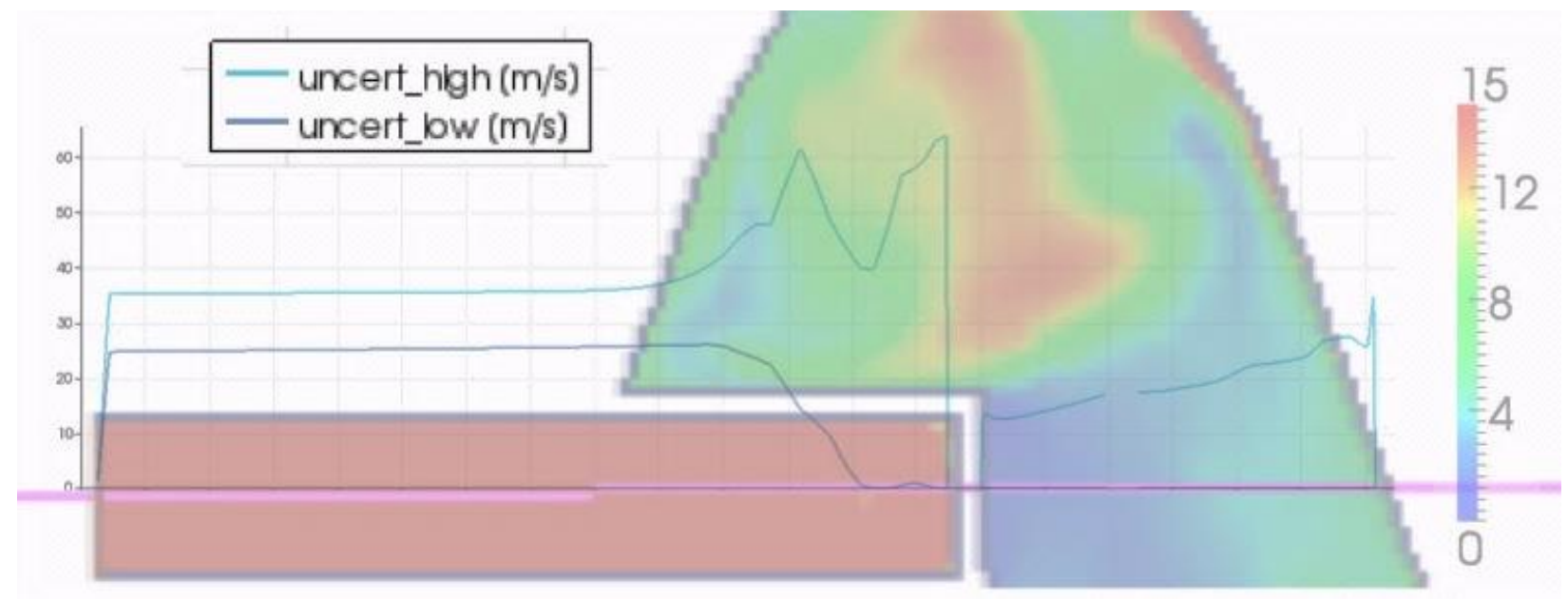

Figure 82: CFD Results and Uncertainty for 5.5m Configuration - Line Plot (m/s)

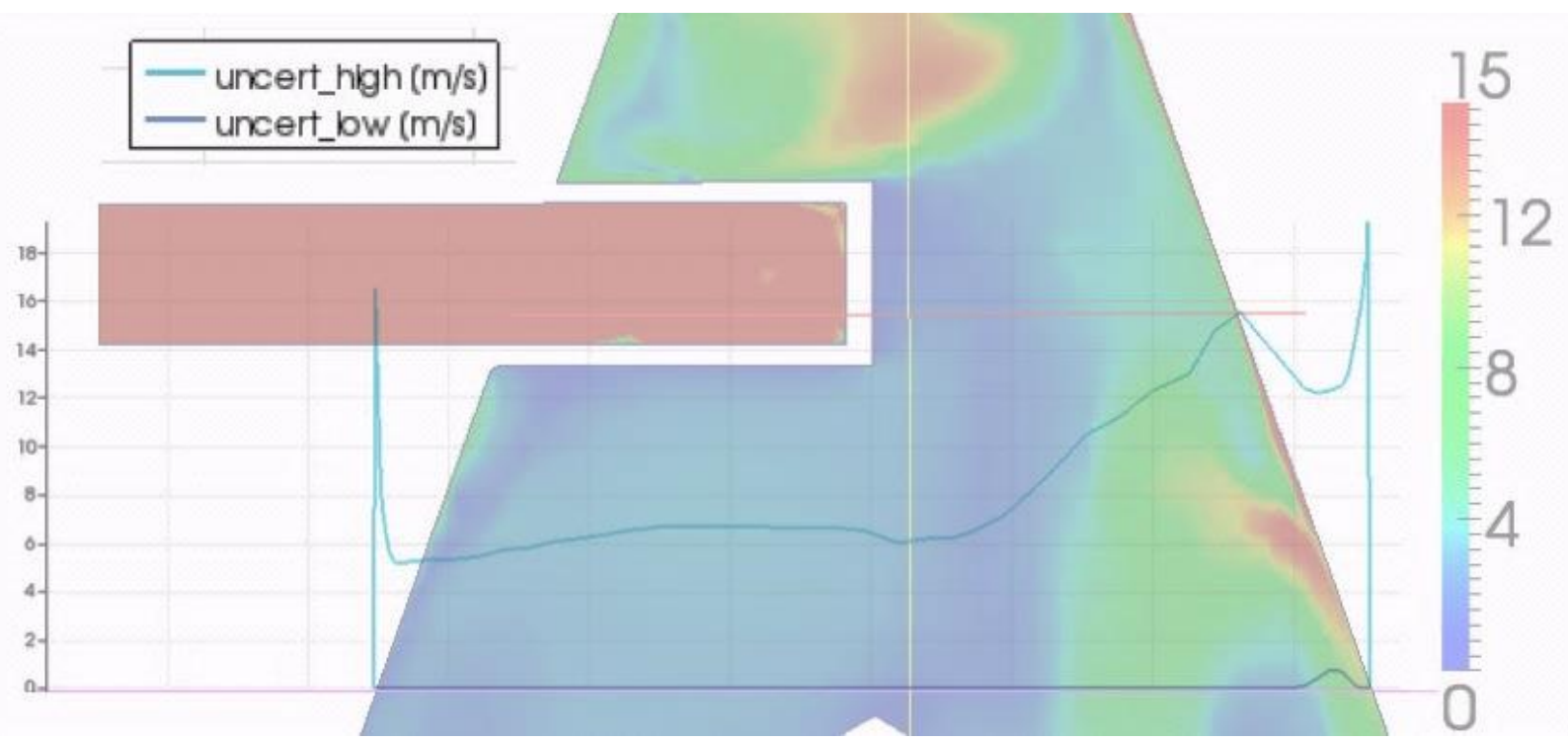

Figure 83: CFD Results and Uncertainty for 5.5m Configuration - Line Plot (m/s) 


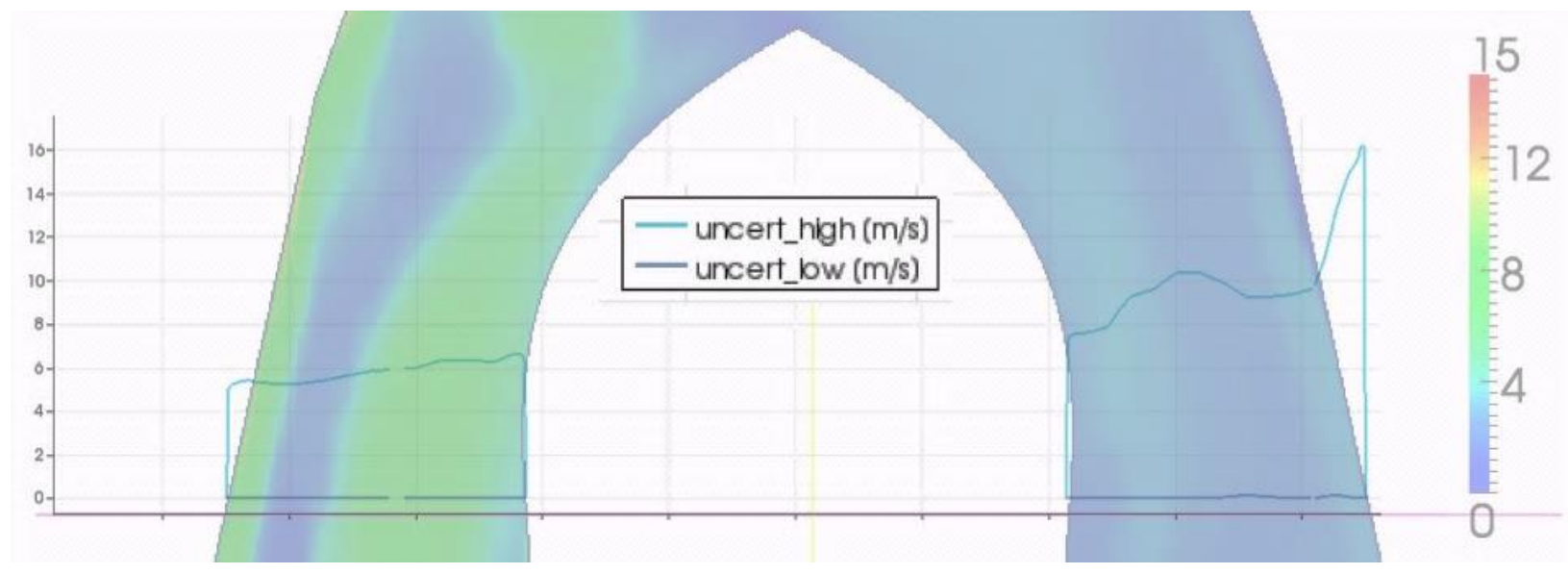

Figure 84: CFD Results and Uncertainty for 5.5m Configuration - Line Plot (m/s)

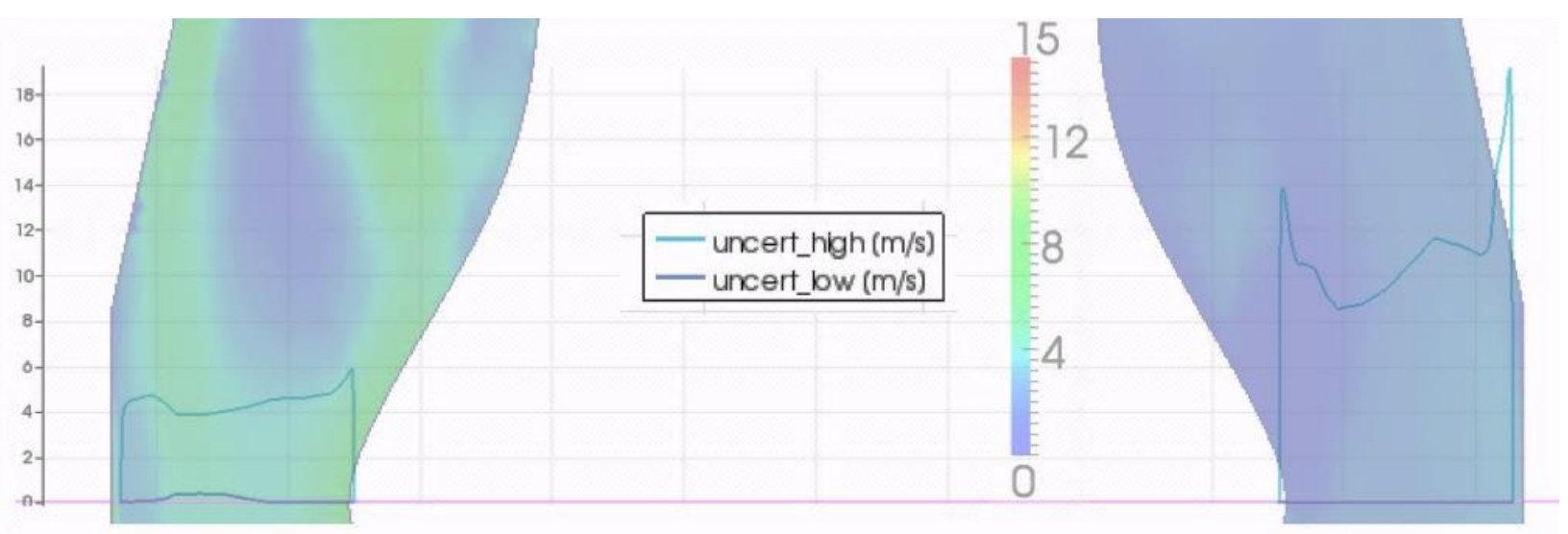

Figure 85: CFD Results and Uncertainty for 5.5m Configuration - Line Plot (m/s)

\subsubsection{Ranking}

The uncertainty for each of the input variables were ranked by the non-dimensionalizing the difference in the results by the freestream value and ranking from greatest uncertainty to least uncertainty. The results are provided in Table 15. 
Table 15: 5.5m Configuration - Non-Dimensional Ranking of Uncertainty

\begin{tabular}{|c|c|c|c|c|c|c|}
\hline $\begin{array}{c}\text { Input } \\
\text { Variable }\end{array}$ & Description & Bias & $\begin{array}{c}\text { Mean } \\
\text { Velocity } \\
\text { Uncertai } \\
\text { nty }(\mathrm{m} / \mathrm{s})\end{array}$ & $\begin{array}{c}\text { Mean Non- } \\
\text { Dimensionali } \\
\text { zed } \\
\text { Uncertainty }\end{array}$ & $\begin{array}{l}\text { Normalized } \\
\text { Ranking \% }\end{array}$ & $\begin{array}{c}\text { Numbered } \\
\text { Ranking }\end{array}$ \\
\hline Grid & $\begin{array}{c}3 \text { grids } \\
\text { considered }\end{array}$ & & 2.0203 & 0.0673 & 12.44 & 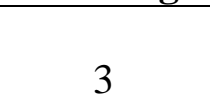 \\
\hline $\begin{array}{c}\text { Inlet } \\
\text { Velocity }\end{array}$ & $\begin{array}{l}\text { Boundary } \\
\text { Condition }\end{array}$ & $10 \%$ & 1.6198 & 0.054 & 9.98 & 6 \\
\hline $\begin{array}{c}\text { Outlet } \\
\text { Pressure }\end{array}$ & $\begin{array}{l}\text { Boundary } \\
\text { Condition }\end{array}$ & $2 \%$ & 2.0173 & 0.0672 & 12.42 & 4 \\
\hline $\begin{array}{l}\text { Turbulenc } \\
\text { e Model }\end{array}$ & $\begin{array}{l}\text { SA, ke- } \\
\text { realizable, } \\
\text { kwSST }\end{array}$ & & 2.3049 & 0.0768 & 14.19 & 1 \\
\hline $\begin{array}{c}\text { Wall } \\
\text { Functions }\end{array}$ & $\begin{array}{l}\text { with and } \\
\text { without }\end{array}$ & & 1.4902 & 0.0497 & 9.18 & 7 \\
\hline $\begin{array}{c}\text { Rough } \\
\text { Wall } \\
\text { Function }\end{array}$ & $\begin{array}{l}\text { smooth vs. } \\
\text { rough }\end{array}$ & & 1.4901 & 0.0497 & 9.18 & 8 \\
\hline $\begin{array}{c}\text { Compress } \\
\text { ibility }\end{array}$ & $\begin{array}{l}\text { incompressi } \\
\text { ble vs. } \\
\text { compressibl } \\
\text { e }\end{array}$ & & 1.4256 & 0.0475 & 8.78 & 9 \\
\hline Solver & $\begin{array}{c}\text { OpenFoam, } \\
\text { Fluent, } \\
\text { STARCCM } \\
+\end{array}$ & & 1.8172 & 0.0606 & 11.20 & 5 \\
\hline $\begin{array}{c}\text { Fluid } \\
\text { Properties }\end{array}$ & $\begin{array}{l}\text { kinematic } \\
\text { viscosity nu } \\
\text { represents } \\
\text { air [0-50- } \\
100] \text { deg C }\end{array}$ & $\begin{array}{c}1.36,1.5 \\
, 2.306 \mathrm{e}- \\
05\end{array}$ & 2.05 & 0.0683 & 12.62 & 2 \\
\hline
\end{tabular}

\subsubsection{Discussion}

The top five most significant input variables in regard to the uncertainty calculation were turbulence model, fluid properties, grid, outlet pressure boundary condition, and solver. This is a 
different trend than the $0.75 \mathrm{~m}$ or $3.5 \mathrm{~m}$ configurations, however the numerical values on the same order of magnitude.

\subsection{ECS System Experimental Comparison}

The problem shown in references [1], [2], [23], and [24] is the only published result prior to this research of the ECS airflow problem. The author was able to receive the CAD models and test data from reference [1], [2], [23], and [24]. CFD models were constructed from the provided CAD models via Pro/ENGINEER, SnappyHex Mesh, and OpenFOAM. The CFD results of the proposed uncertainty methodology were compared to the test data 'blind'. The variables considered in the uncertainty analysis were inlet velocity, viscosity, outlet pressure, and turbulence. The inlet velocity and outlet pressure were assumed to have a bias error of 3 percent. The kinematic viscosity used was varied from $1.36 \mathrm{e}-5$ to $2.306 \mathrm{e}-5$ to account for all temperature ranges between 0 and 100 degrees Celsius. The turbulence models considered were Spalart Allmaras, ke-realizable, and kwsst. The confidence level was assumed to be 90 percent and a k factor of 1.86 was used. The results are presented for the measurement locations in reference 15 and 16. The Uy and Uz velocity components were compared at three locations of constant $\mathrm{x}$ and four locations of constant $\mathrm{z}(\mathrm{x}=7 \mathrm{in}, 8.5 \mathrm{in}$, and $9 \mathrm{in})$ as shown in Figure 86 and $(\mathrm{z}=-5$ in, $-3 \mathrm{in},-2 \mathrm{in}$, and 0 in) as shown in Figure 87. 

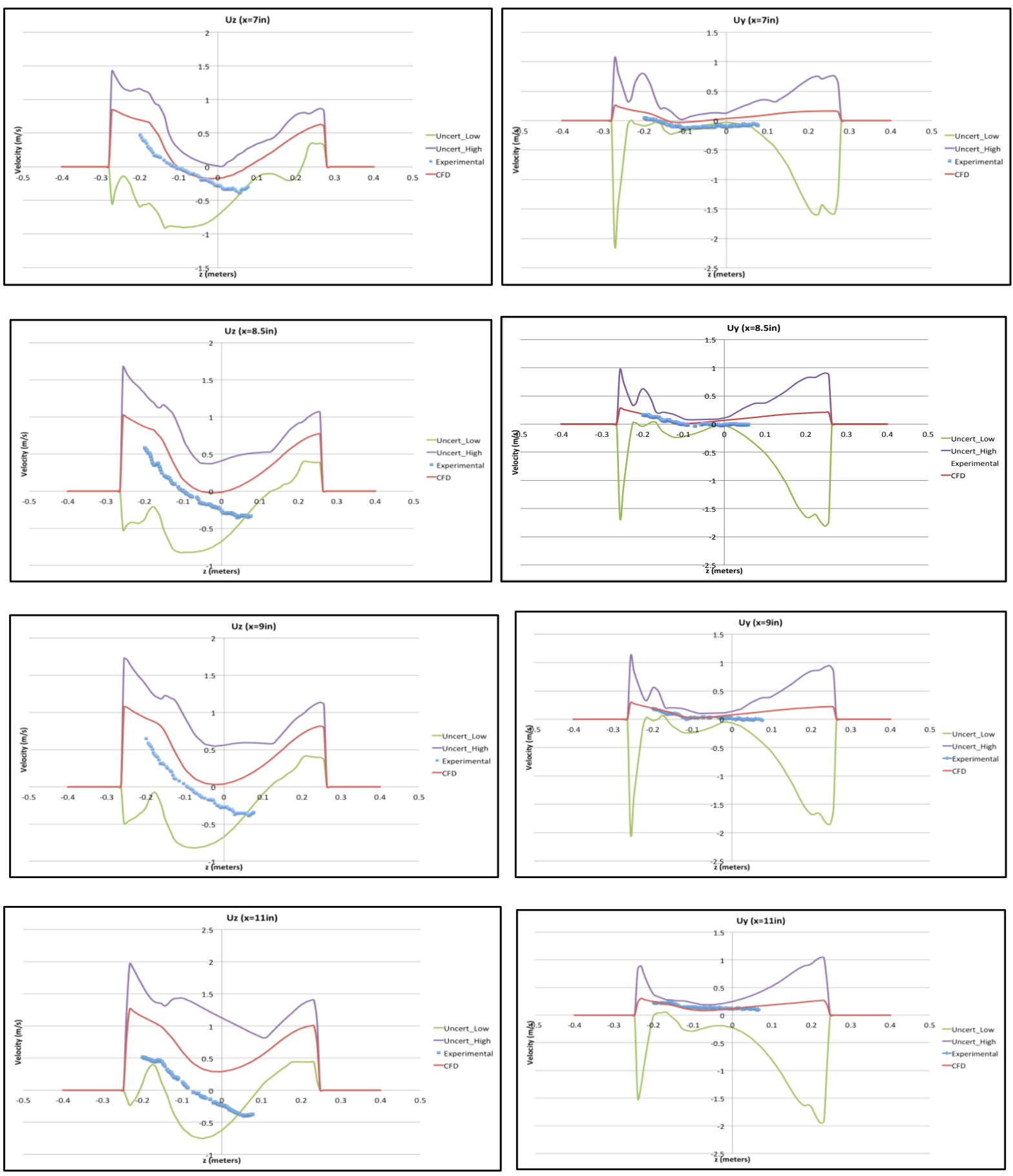

Figure 86: Proposed CFD Uncertainty Method vs. Experimental Data constant x 

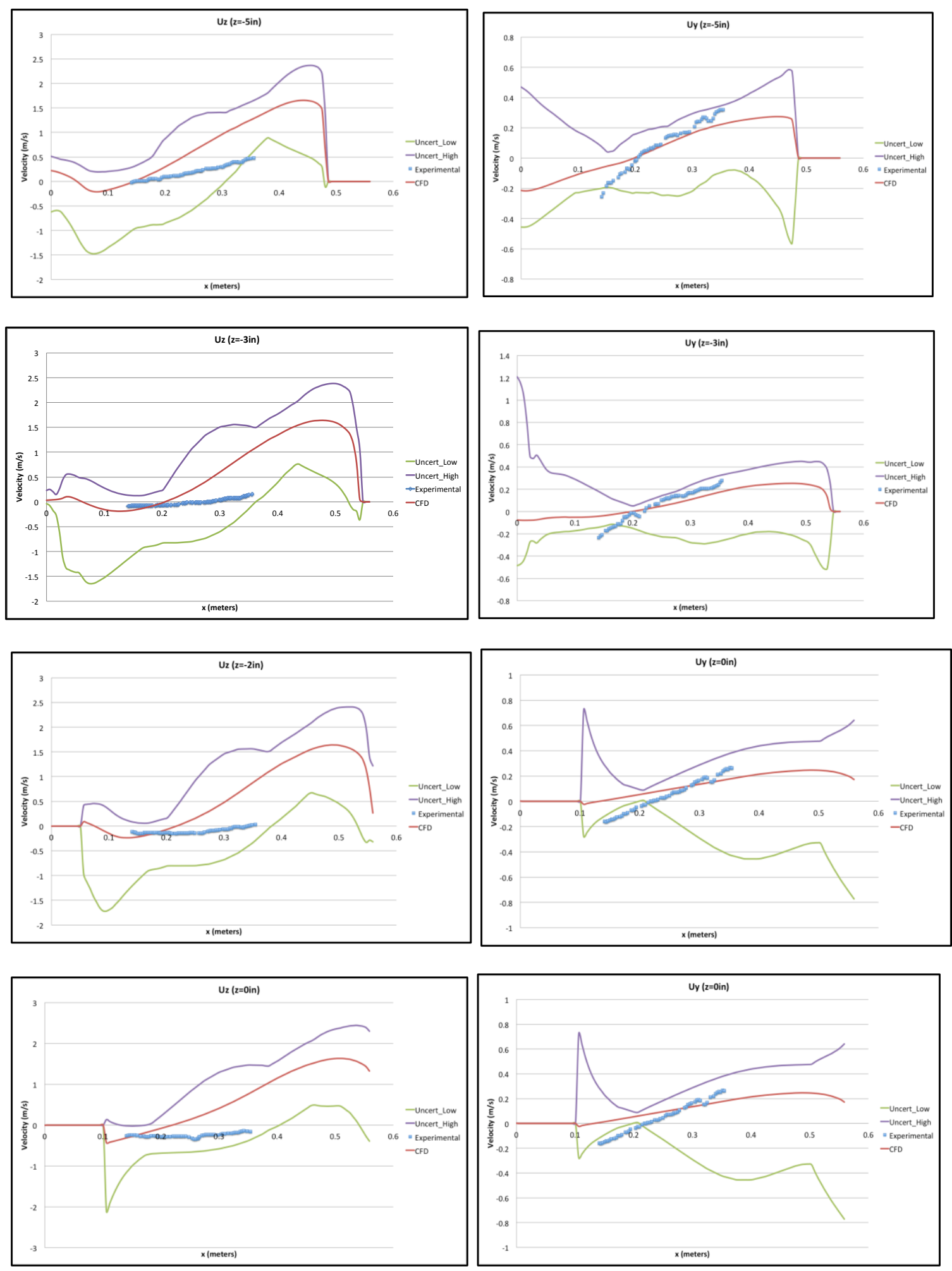

Figure 87: Proposed CFD Uncertainty Method vs. Experimental Data constant z 
The proposed methodology for the limited set of test data encompassed most of the results. There were a total of 1,085 points measured and 977 of those were inside the $90 \%$ CFD Uncertainty methodology. This further validates the proposed methodology. Nighty percent of the experimental data lies within the $90 \%$ proposed CFD methodology. The CFD model and testing apparatus is shown in Figure 88.

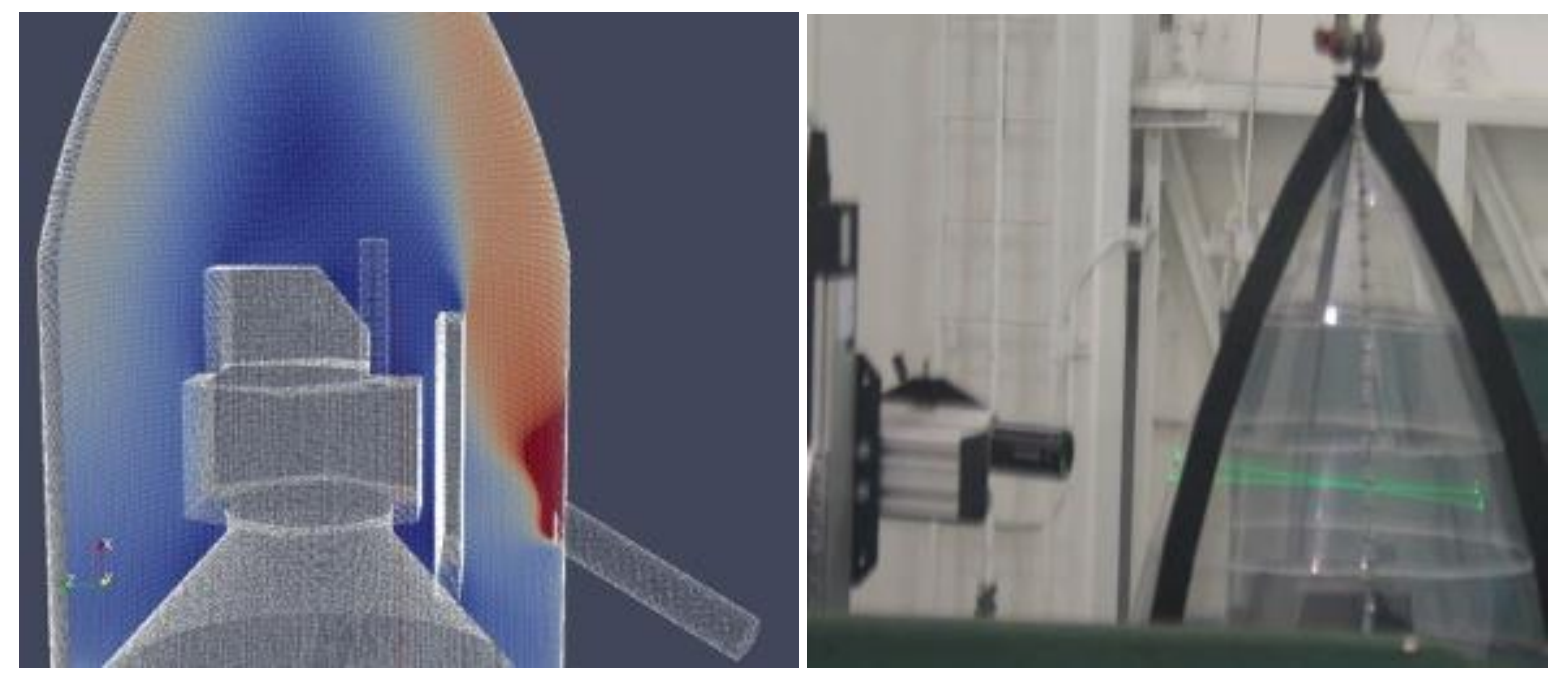

Figure 88: CFD Model and Experimental Apparatus [24]

This section provided uncertainty estimates for the three generic configurations, demonstrated the methodology, and proved the approach as compared to experimental data. 


\section{CHAPTER SEVEN: CONCLUSIONS}

Industry is using Computational Fluid Dynamics to predict airflow fields without proper uncertainty calculations or validations. The purpose of this research is to propose a method to estimate the uncertainty in a Computational Fluid Dynamics (CFD) model using only numerical simulations and without any test data. This method is not designed to eliminate the need for testing or proper validation. The method strictly provides an uncertainty estimate when test data and validation are not obtainable.

The proposed method draws from industry standards in experimental and numerical model validation and couples the methods to provide a calculation parallel to traditional uncertainty predictions. There were two items learned from performing the uncertainty calculation of flow over a backward facing step. The first, Richardson's extrapolation method cannot be used for the entire computational domain since the flow velocity is not always monotonically increasing or decreasing. Two, when interpolating between grids, a 'linear' interpolation method must be used.

The following two simple examples were considered: the exact solution of laminar flow between parallel plates and experimentally derived turbulent heat transfer coefficients. The proposed methodology encompassed the uncertainty prediction from the 'traditional' way of performing these calculations using only CFD models for both cases considered. Additionally, it was shown by interpolating all solutions onto the coarse grid can provided higher order of accuracy and less error in the extrapolated solution. 
The problem of interest is environmental control systems which have spacecraft impingement requirements. CFD can be used to verify these requirements, however proper validation and experimental data should be used to anchor the CFD predictions. If the data is not available or obtainable, the proposed methodology couples the Student-T distribution to the number of CFD models and input parameters to provide a realistic uncertainty prediction. The cases considered are representative of the EELV fleet, however the methodology has only been experimentally compared to a single set of data. Future work should consider performing an experiment of one of the configurations considered and compare the experimental data to the proposed methodology. Additionally, the flow regime considered in this paper was internal, low speed, steady, incompressible air. The method has not been verified for other flow regimes which are dominated by compressibility or any other factor. Future work should consider extending the proposed methodology to external flows, compressible flows, unsteady flows, and other disciplines. It is believed the method could be applied to any discipline or numerical simulation.

It was hypothesized for the ECS spacecraft configurations that certain input variables would be negligible in the overall uncertainty calculation and through an order of magnitude analysis may be neglected. This hypothesis was proved false for the following input parameters: Grid, Inlet Velocity, Outlet Pressure, Turbulence Model, Wall Functions, Compressibility, Solver, and Fluid Properties. Each of these inputs should be considered and have the same order of magnitude importance to the overall uncertainty estimate.

Based off of all the models and data considered, it is recommended all input variables in Table 11 and all seventeen cases in Table 12 be considered along with the corresponding $\mathrm{k}$ factor in Table 
1 to provide an uncertainty estimate for CFD when proper testing and validation cannot be performed. 


\section{APPENDIX A: INTERPOPENFOAM.M}




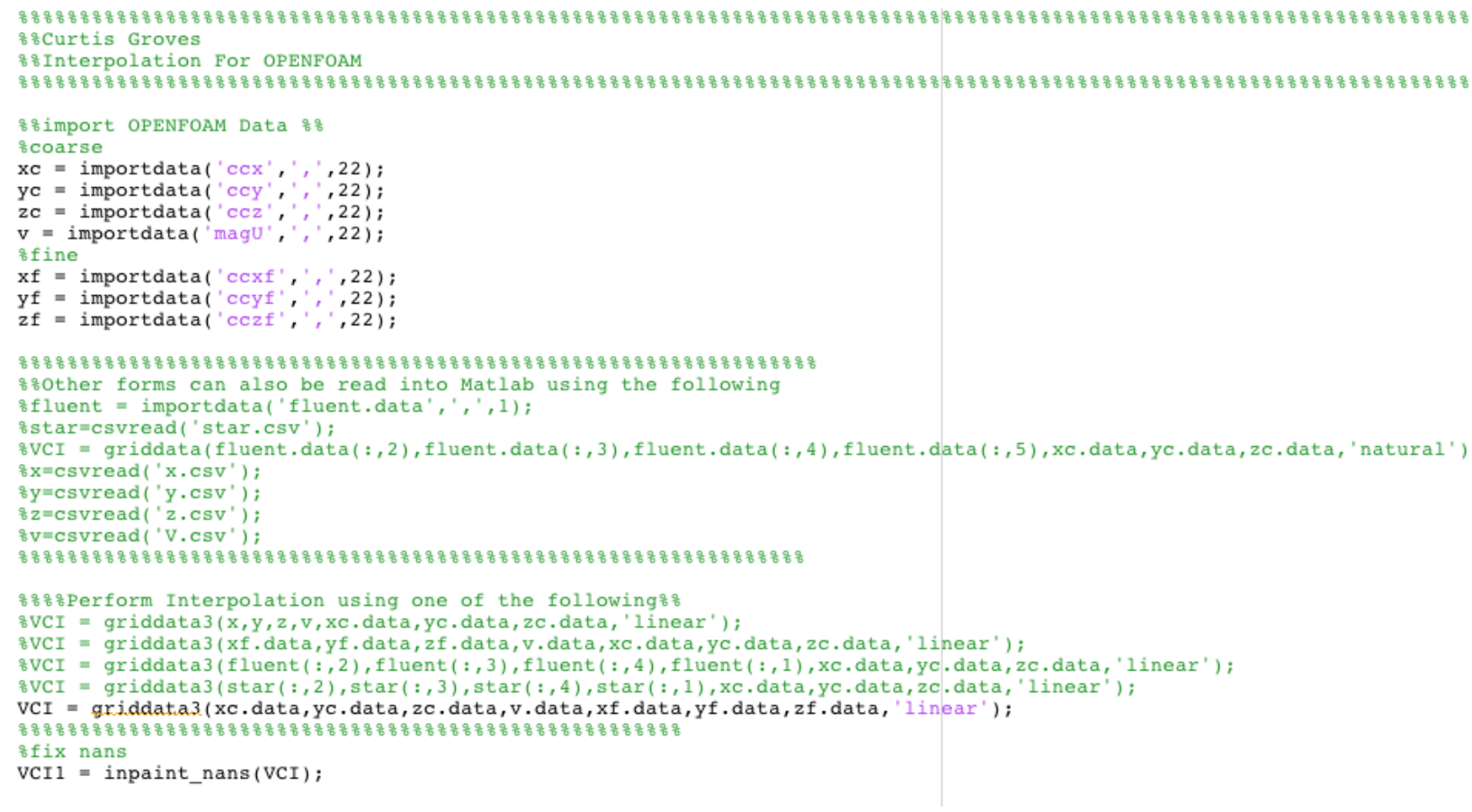




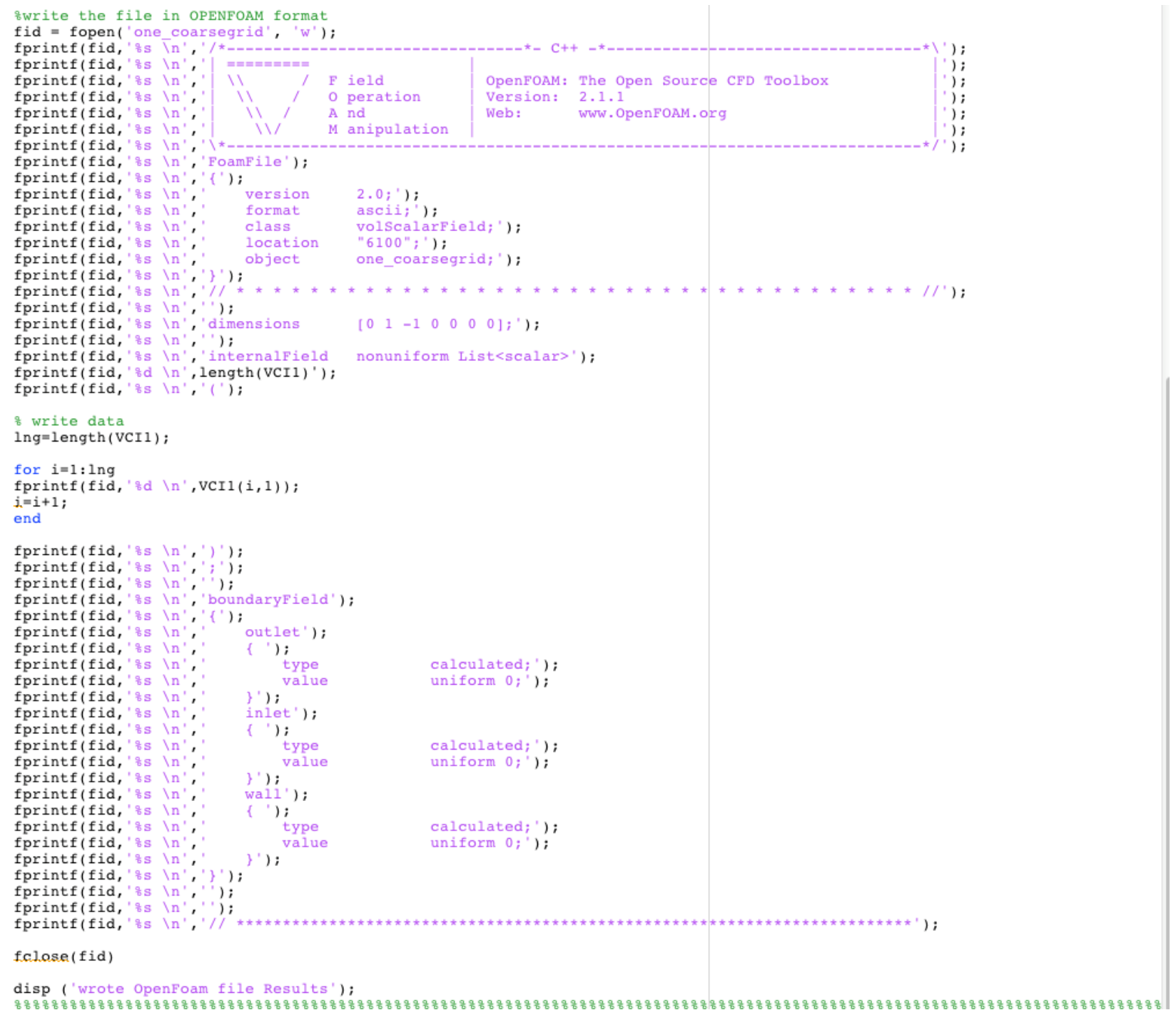


APPENDIX B: UNCERTOPENFOAM.M 


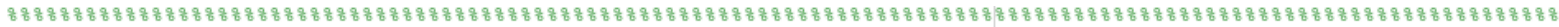
은

을 Uncertainty Analysis For OPENFOAM

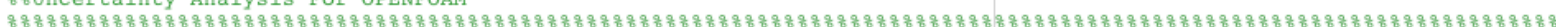

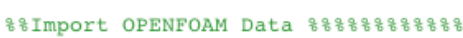

one_coarsegrid = importdata('one_coarsegrid' ,' ', '22);

two_medgrid = importdata('two_medgrid',', '22); '

three_finegrid = importdata('three finegrid',',22);

four_ulow = importdata('four ulow', ', ',22);

five_uhigh = importdata('five_uhigh', ', ',22);

six plow = importdata('six plow', ', 22$)$;

seven_phigh $=$ importdata ('seven_phigh', ', ',22):

eight_sa = importdata('eight_sa', ', ',22);

nine_ke = importdata ('nine_ke', ',', 22);

ten kwsst = importdata('ten kwsst', ', 22);

eleven nowallfns $=$ importdata ('eleven nowailfns', ', ', 22)

twelve_roughwall = importdata ('twelve_roughwall', ', ', 22)

thirteen_compr = importdata ('thirteen_compr',', ', 22);

fourteen_fluent importdata ( fourteen_fluent,', ', 22),

fifteen_star = importdata ('fifteen_star, ', 22), '

sixteen_nuthigh = importdata('sixteen_nuthigh', ', 22);

seventeen_nutlow = importdata ('seventeen_nutlow', ', ',22);

save

은 load matlab.mat

of

data $(:, 1)=$ one coarsegrid.data $(:, 1)$

data $(:, 2)=$ two medgrid.data $(:, 1)$;

data $(:, 3)=$ three finegrid.data $(:, 1)$;

data $(:, 4)=$ four ulow.data $(:, 1)$;

data $(:, 5)=$ five uhigh.data $(:, 1)$

data $(:, 6)=\operatorname{six}$ plow.data $(:, 1)$;

data $(:, 7)=$ seven_phigh.data $(:, 1)$

data $(:, 8)=$ eight_sa.data $(:, 1)$

data $(:, 9)=$ nine_ke.data $(:, 1)$;

$\operatorname{data}(:, 10)=$ ten_kwsst.data $(:, 1)$;

data $(:, 11)=$ eleven nowallins.data $(:, 1)$;

data $(:, 12)=$ twelve roughwall.data $(:, 1)$

data $(:, 13)=$ thirteen compr.data $(:, 1)$;

data $(:, 14)=$ fourteen fluent.data $(:, 1)$

data $(:, 15)=$ fifteen_star.data $(:, 1)$;

data $(:, 16)=$ sixteen_nuthigh.data $(:, 1)$;

data $(:, 17)=$ seventeen_nutlow.data $(:, 1)$;

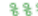


Appendix B.1 Uncertopenfoam.M -Max,Min,Uncert 


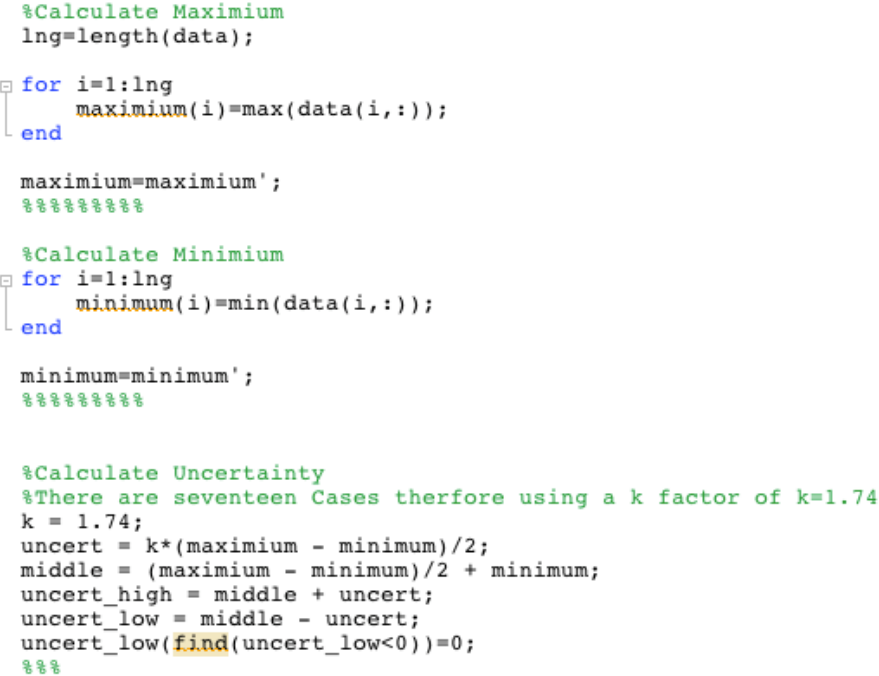


Appendix B.2 Uncertopenfoam.M - Write Min 


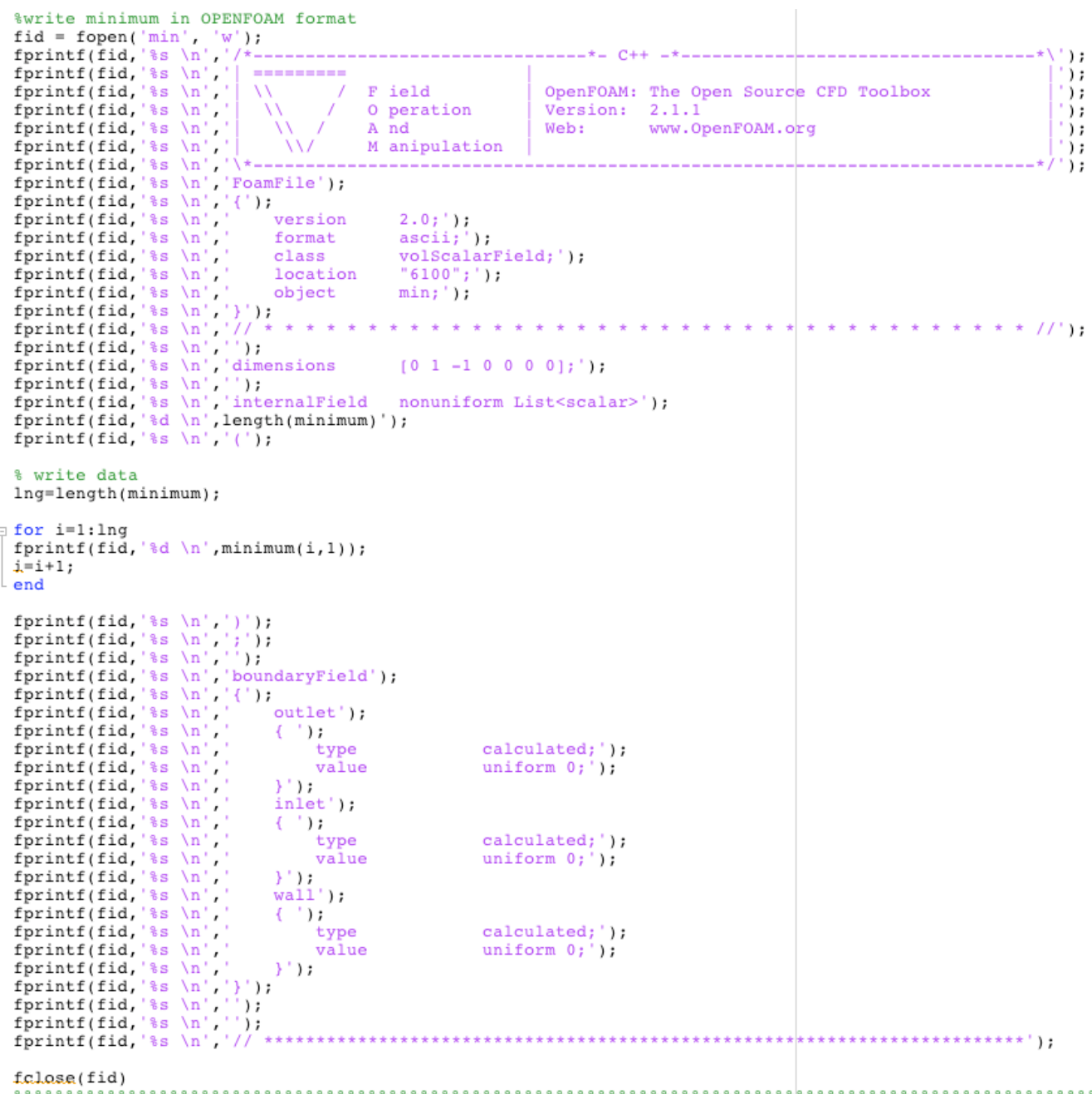

fclose(fid)

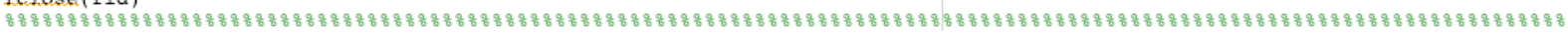


Appendix B.3 Unceropenfoam.M - Write Max 


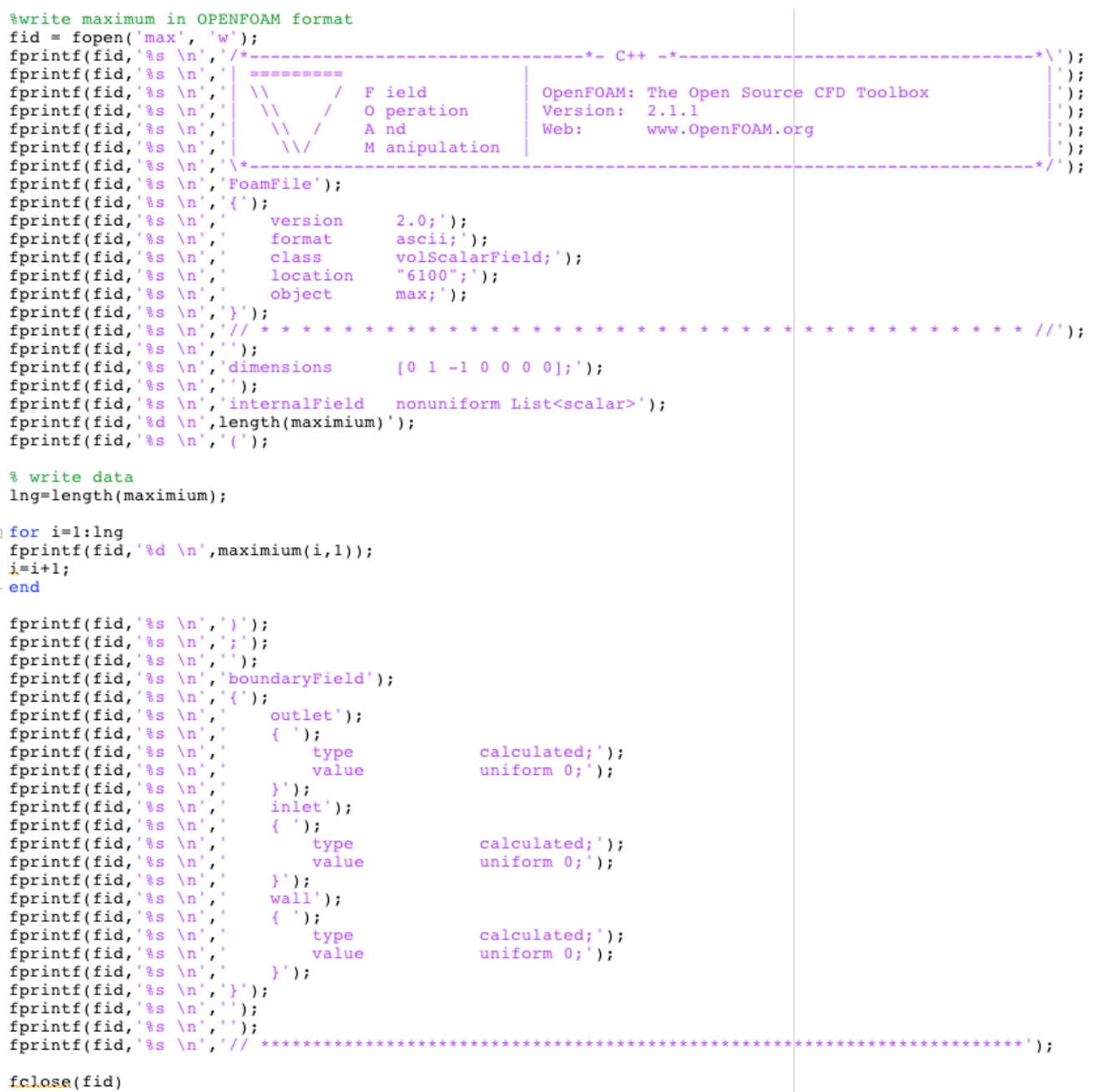

fclose(fid)

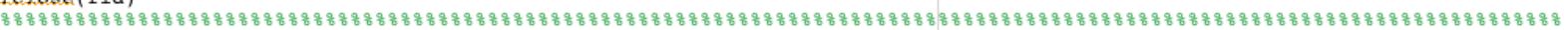


Appendix B.4 Uncertopenfoam.M -Write Middle 


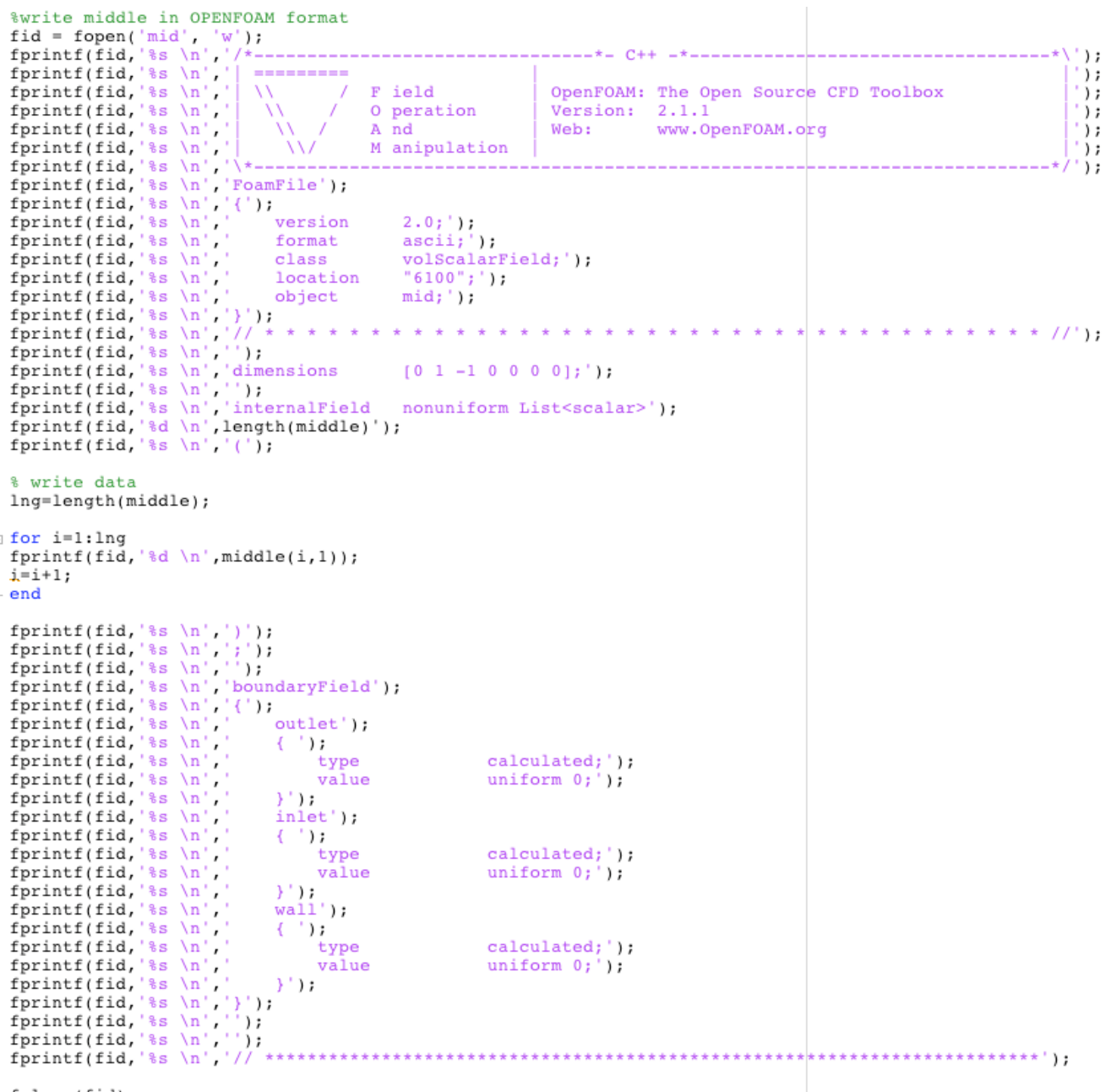

falose(fid)

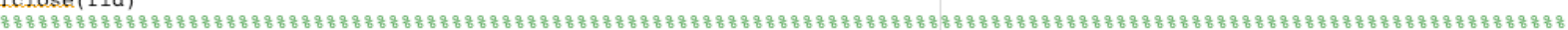


Appendix B.5 Uncertopenfoam.M - Write Uncertainty 


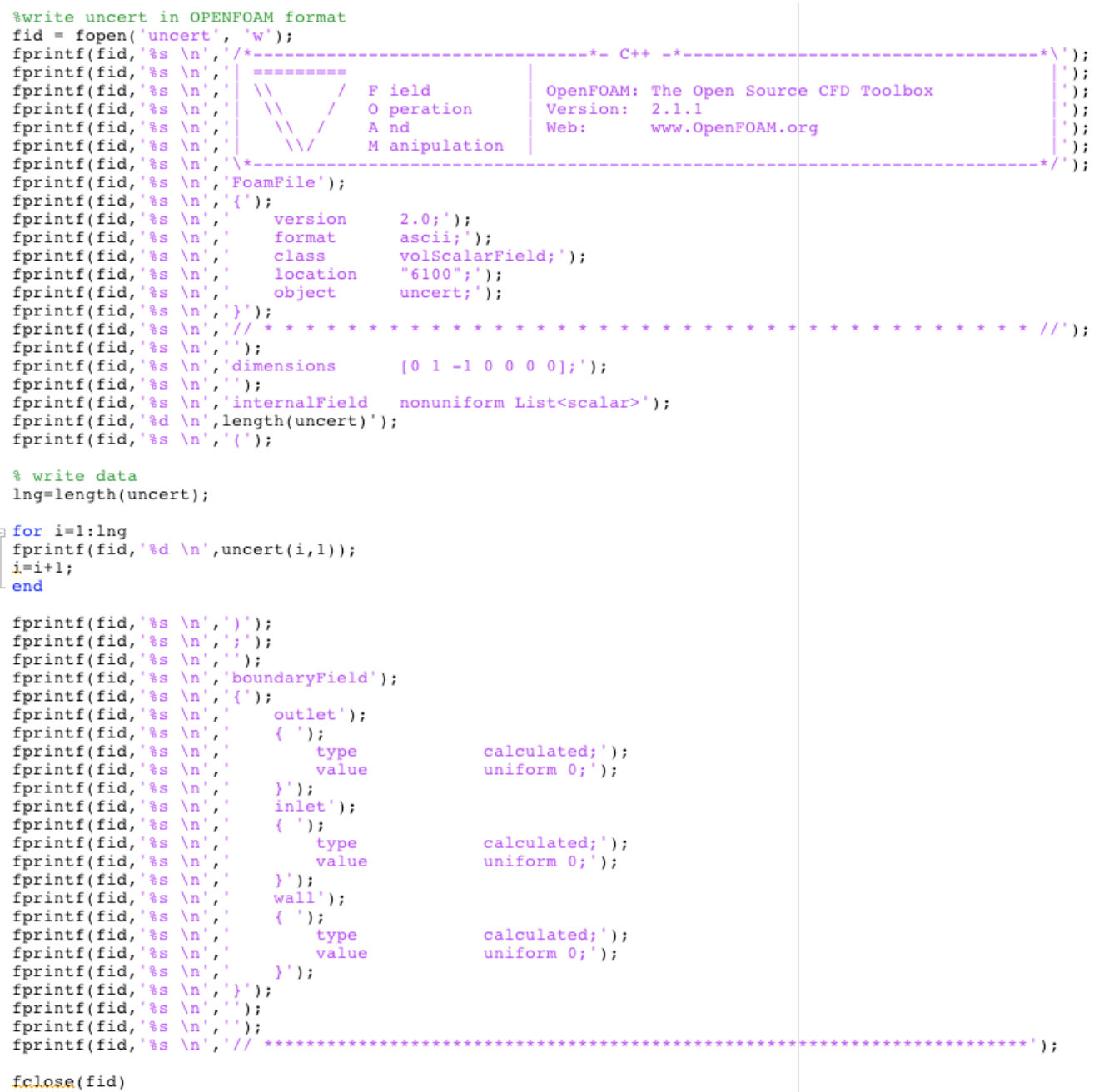

falose(fid)

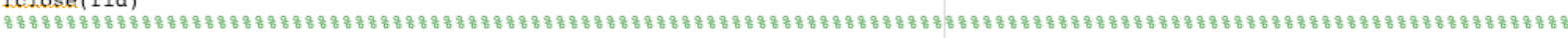


Appendix B.6 Uncertopenfoam.M - Write Uncertainty Low 


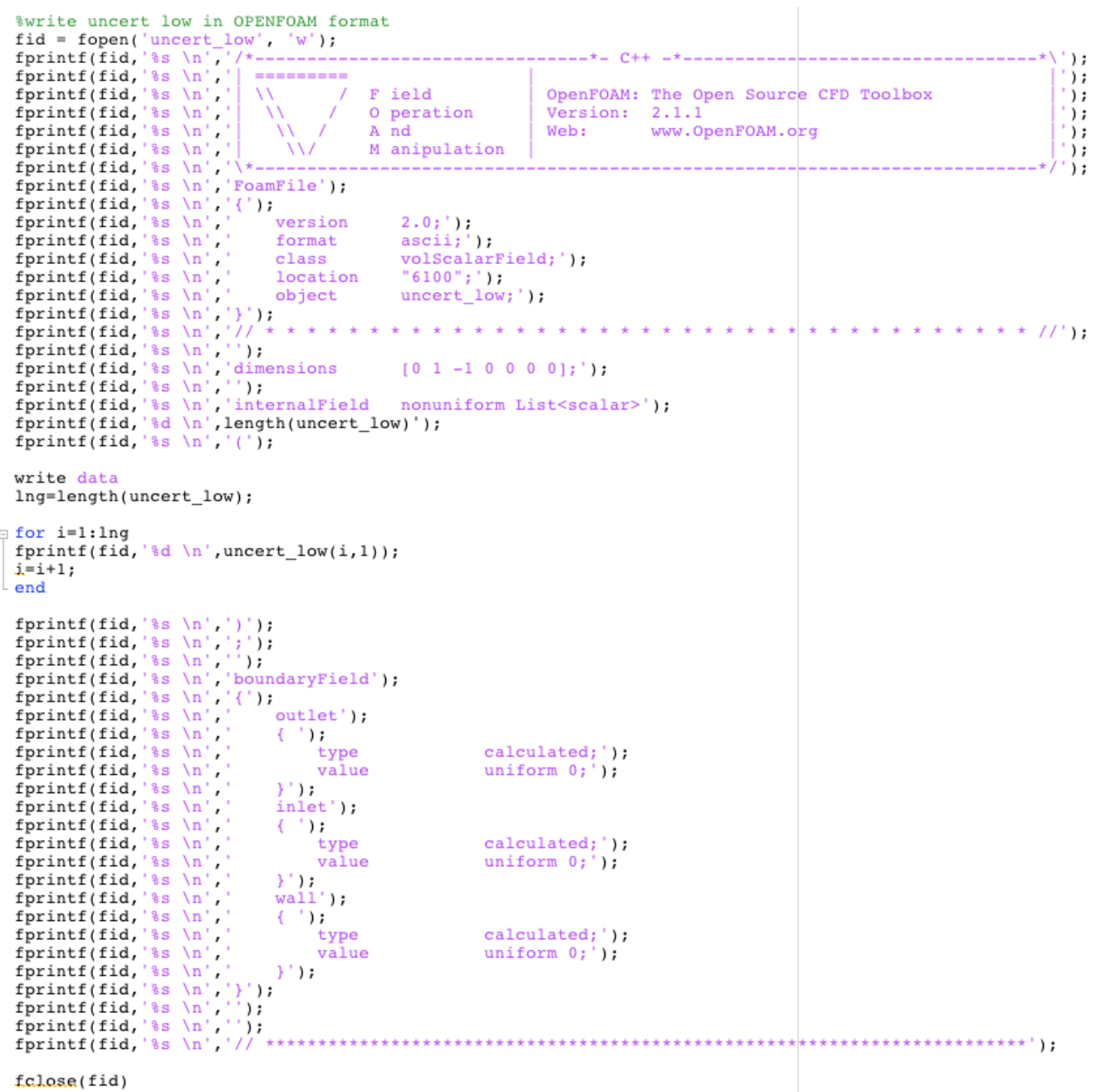

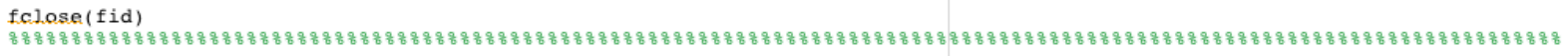


Appendix B.7 Uncertopenfoam.M - Write Uncertainty High 


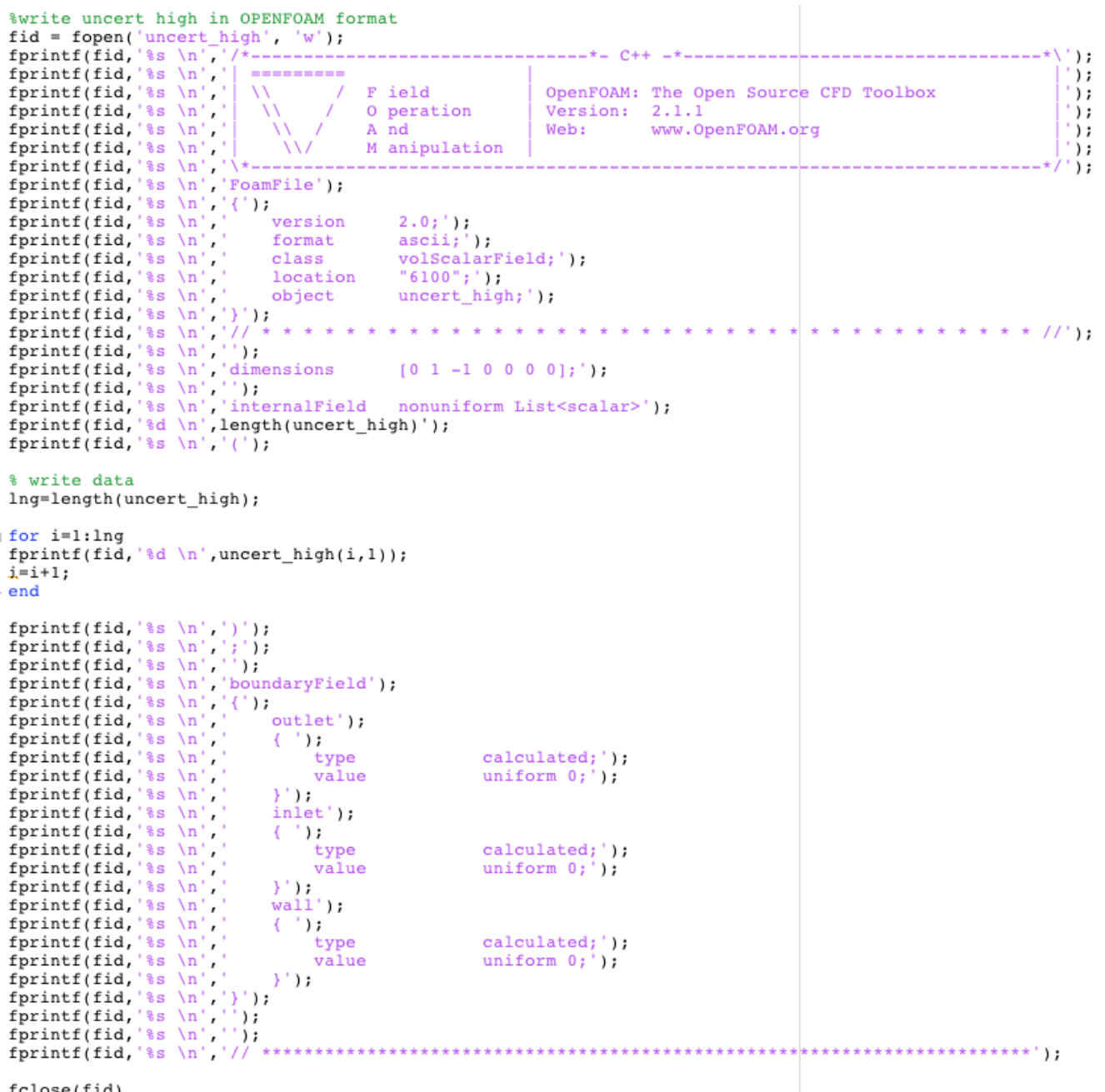

fclose(fid)

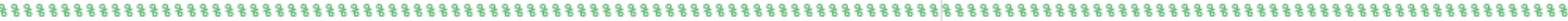




\section{APPENDIX C: RKOPENFOAM.M - CALCULATE RK AND WRITE OPENFOAM}


은 Compute Rk Values are write OPENFOAM RK File

load matlab.mat

e_21 = two_medgrid.data $(:, 1)$ - three_finegrid.data $(:, 1)$

$e^{-} 32$ one_coarsegrid.data $(:, 1)$ - two_medgrid.data $(:, 1)$;

$\mathrm{R} \overline{\mathrm{k}}=\mathrm{e} 21 . \mathrm{e} 32 ;$

$\operatorname{Rk}(\sim \operatorname{is} \bar{f}$ inite $\overline{(R k}))=0$;

swrite uncert low in OPENFOAM format

fid = fopen('Rk', 'W');

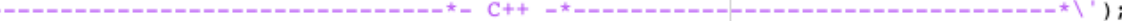

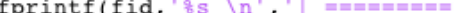

fprintf(fid, 'zs $\backslash n$ '

,

fprintf(fid,' 's $\backslash n$

fprintf(fid, ' to $\backslash \mathrm{n}$ ',

fprintf(fid, '

fprintf(fid, os

version $2,0,1,{ }^{\prime}$

fprintf(fid, 'os $\backslash n^{\prime}$, format ascii;')

fprintf(fid, 'o $\backslash \mathrm{n}^{\prime}, \mathrm{y}^{\prime}$ class volscalarField;')

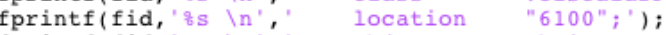

fprintf(fid, 'ㅇs $\backslash \mathrm{n}^{\prime},{ }^{\prime}$ object Rk;')

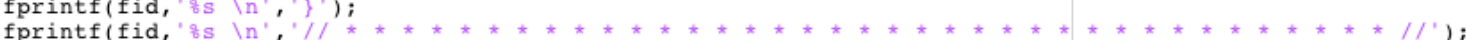

fprintf(fid, 'os $\backslash \mathrm{n}$ ', '');

fprintf(fid, 'os $\backslash \mathrm{n}$ ', 'dimensions $\quad\left[\begin{array}{lllllll}0 & 1 & -1 & 0 & 0 & 0 & 0\end{array}\right] ;$ ');

fprintf(fid, 'os $\backslash n$ ', '');

fprintf(fid, ' ts $\backslash \mathrm{n}^{\prime}$,' internalField nonuniform List<scalar>');

fprintf(fid, ' o d $\backslash \mathrm{n}^{\prime}$, length(Rk)');

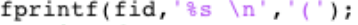

\% write data

lng=length (Rk),

for $i=1:$ lng

fprintf(fid, ' od $\left.\backslash n^{\prime}, \operatorname{Rk}(i, 1)\right)$;

$j .=i+1$

end

fprintf(fid, 'os $\left.\backslash \mathrm{n}^{\prime},{ }^{\prime}\right)$ ')

fprintf(fid, 'ots $\left.\backslash \mathrm{n}^{\prime},{ }^{\prime} ;{ }^{\prime}\right)$;

fprintf(fid, 'os $\backslash \mathrm{n}$ ', '');

fprintf(fid,' 's $\backslash \mathrm{n}^{\prime}$ ', 'boundaryField');

fprintf(fid, 'os $\backslash \mathrm{n}^{\prime}$, ' $^{\prime}$ ');

fprintf(fid, 'os $\backslash \mathrm{n}^{\prime}$, , $^{\prime}$ outlet');

fprintf(fid, o⿱ $\mathrm{s} \backslash \mathrm{n}, \quad\{$ );

fprintf(fid, 'os $\backslash n^{\prime}, a^{\prime}$ ( ) ;

calculated; '):

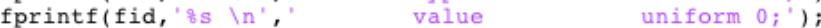

fprintf (fid, ' o s $\left.\backslash n^{\prime}, ' \quad\right\}$ ');

fprintf(fid, 'os $\backslash \mathrm{n}^{\prime}$, ' inlet');

fprintf(fid, ' os $\backslash \mathrm{n}^{\prime}$, ' $\{$ ');

fprintf(fid, 'o $\mathrm{s} \backslash \mathrm{n}^{\prime}$,'

fprintf(fid, 'ㅇs $\backslash \mathrm{n}^{\prime}$, ',

fprintf(fid, ' $\mathrm{t} s \backslash \mathrm{n}$ ',

fprintf(fid, ' ts $\backslash \mathrm{n}^{\prime}$, '

fprintf(fid, ' ts $\backslash \mathrm{n}^{\prime}$,'

fprintf(fid, ' os $\backslash n^{\prime}$,'

fprintf(fid, 'os $\backslash \mathrm{n}^{\prime}$,'

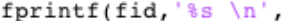

fprintf(fid, 'os $\left.\left.\backslash \mathrm{n}^{\prime},{ }^{\prime}\right\}^{\prime}\right)$;

fprintf(fid, '호 $\backslash \mathrm{n}^{\prime}, \mathrm{n}^{\prime}$ ');

fprintf(fid, 'os $\backslash \mathrm{n}$ ', ',

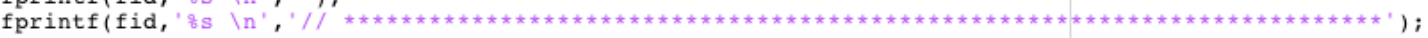

falose (fid)

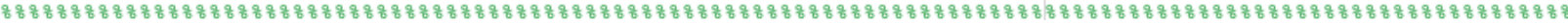




\section{APPENDIX D: NONDIMRANK.M - NON-DIMENSIONALIZATION AND}

\section{RANKING OF INPUT UNCERTAINTY}




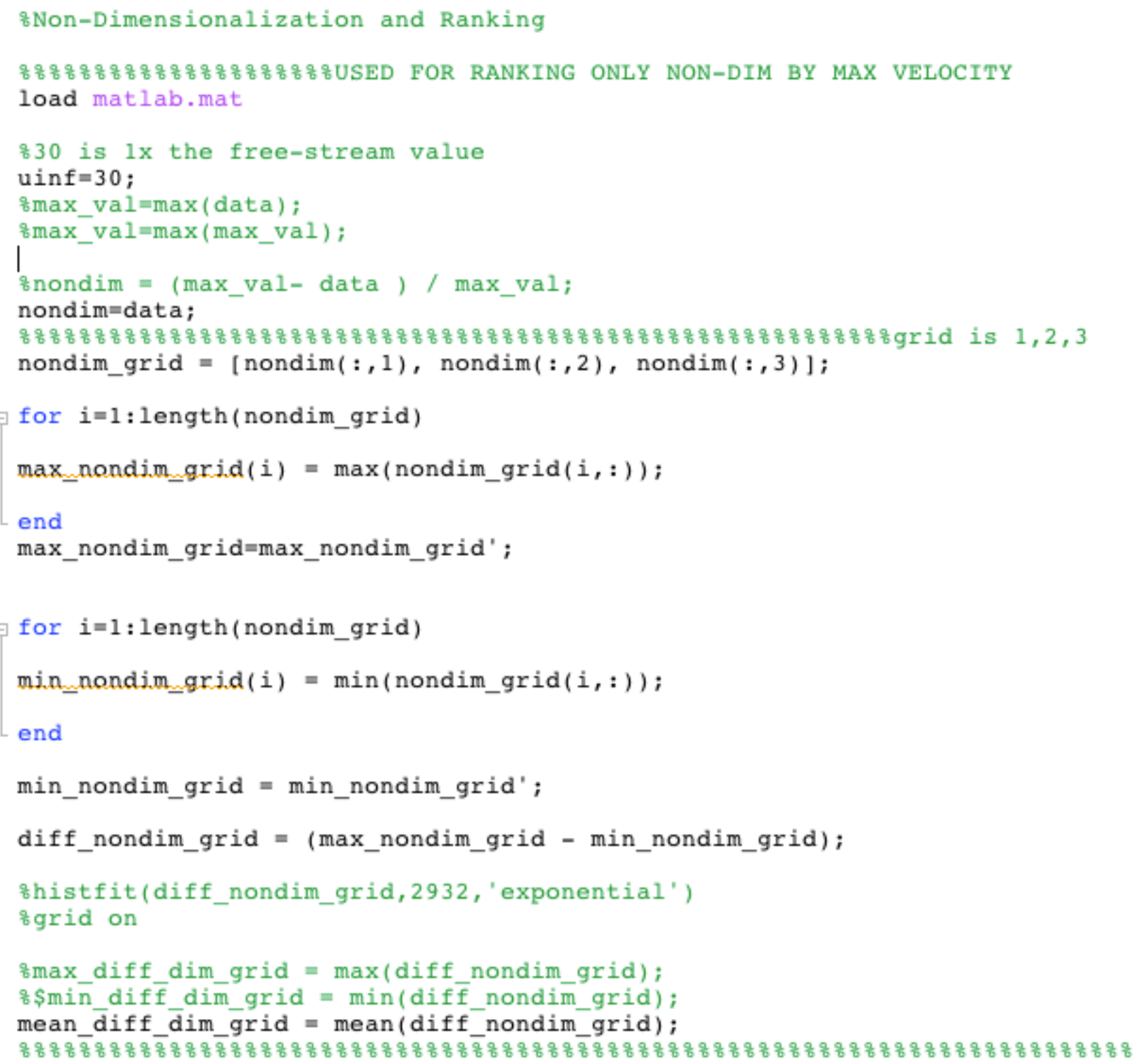




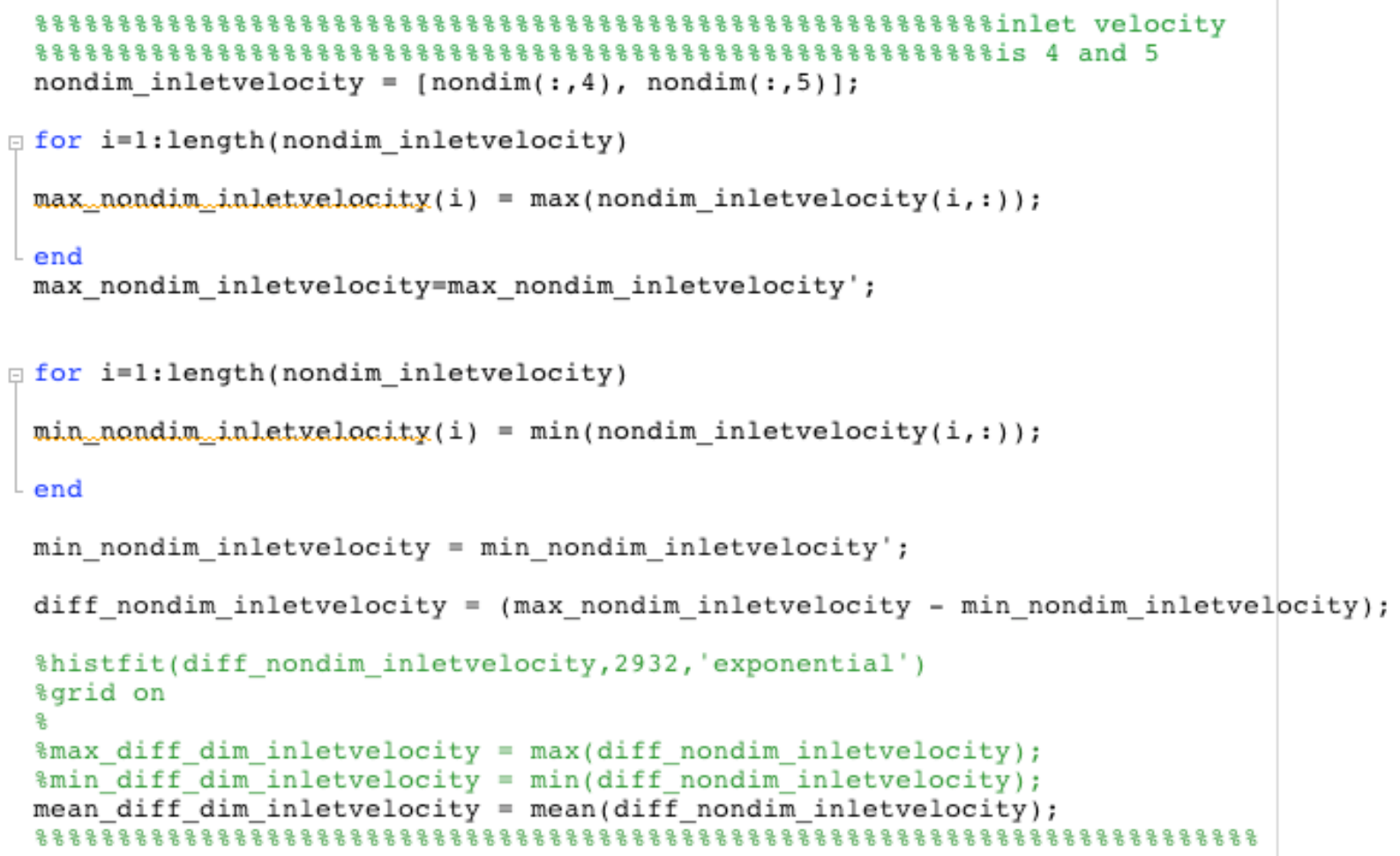




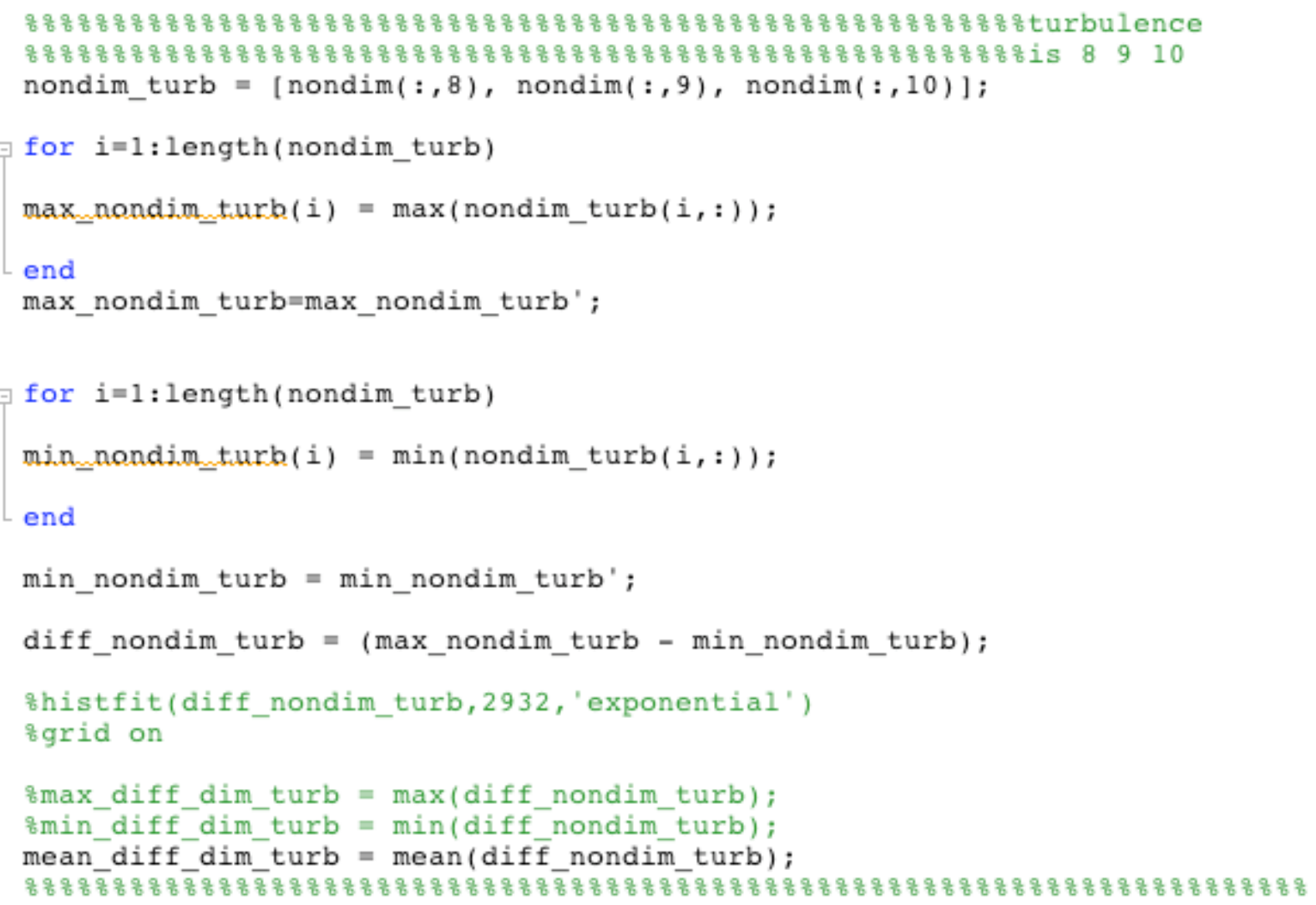




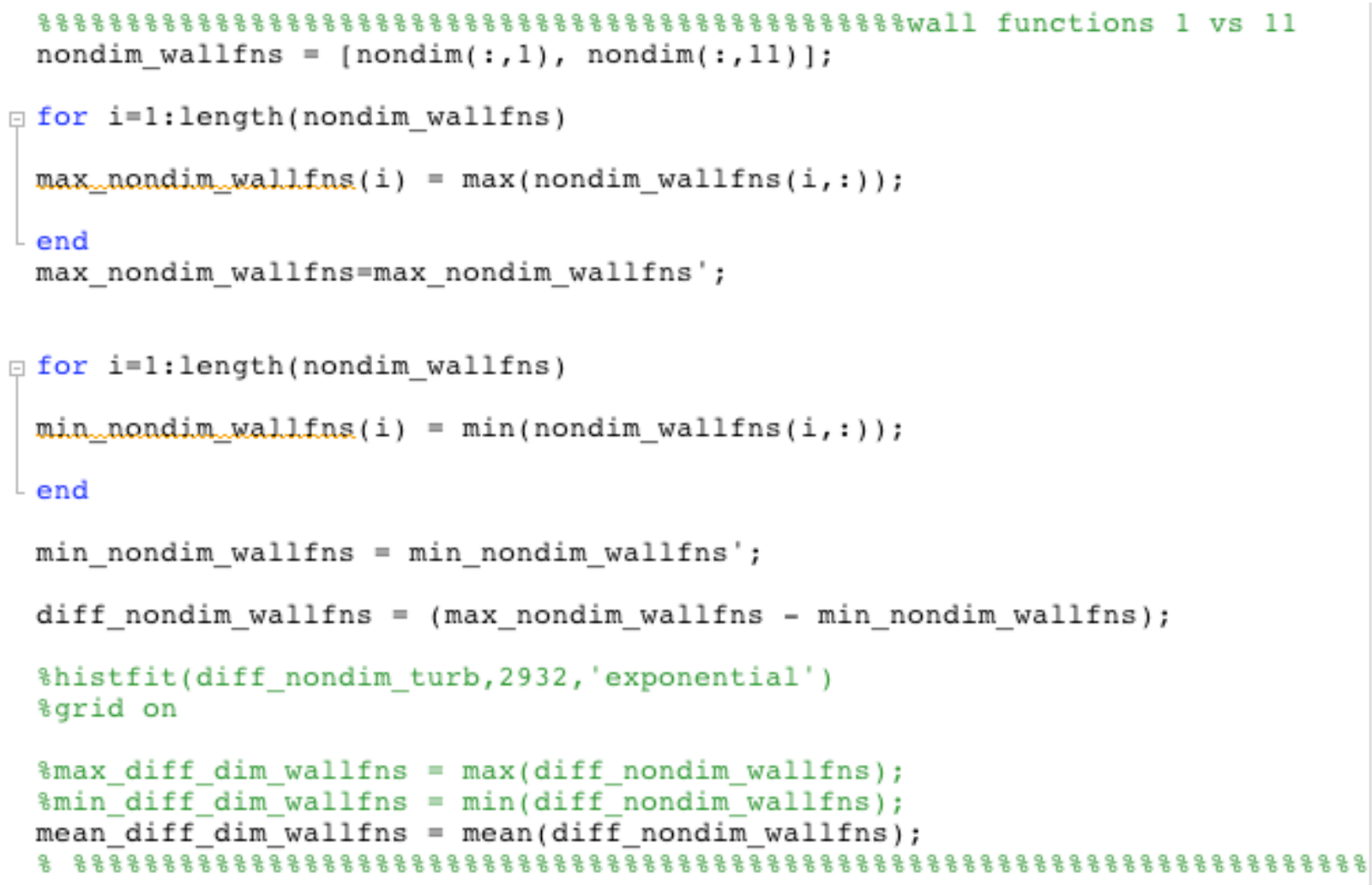




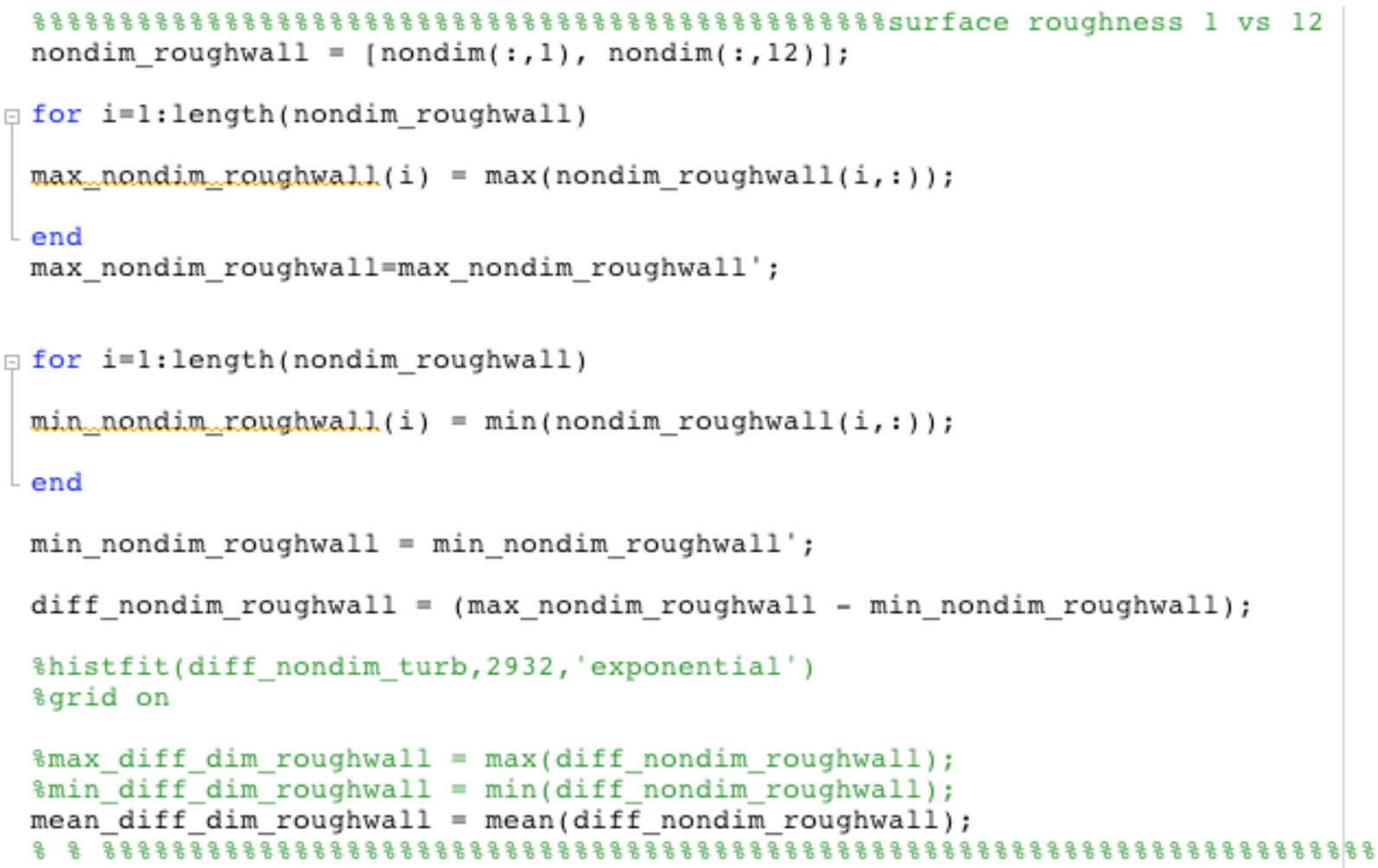




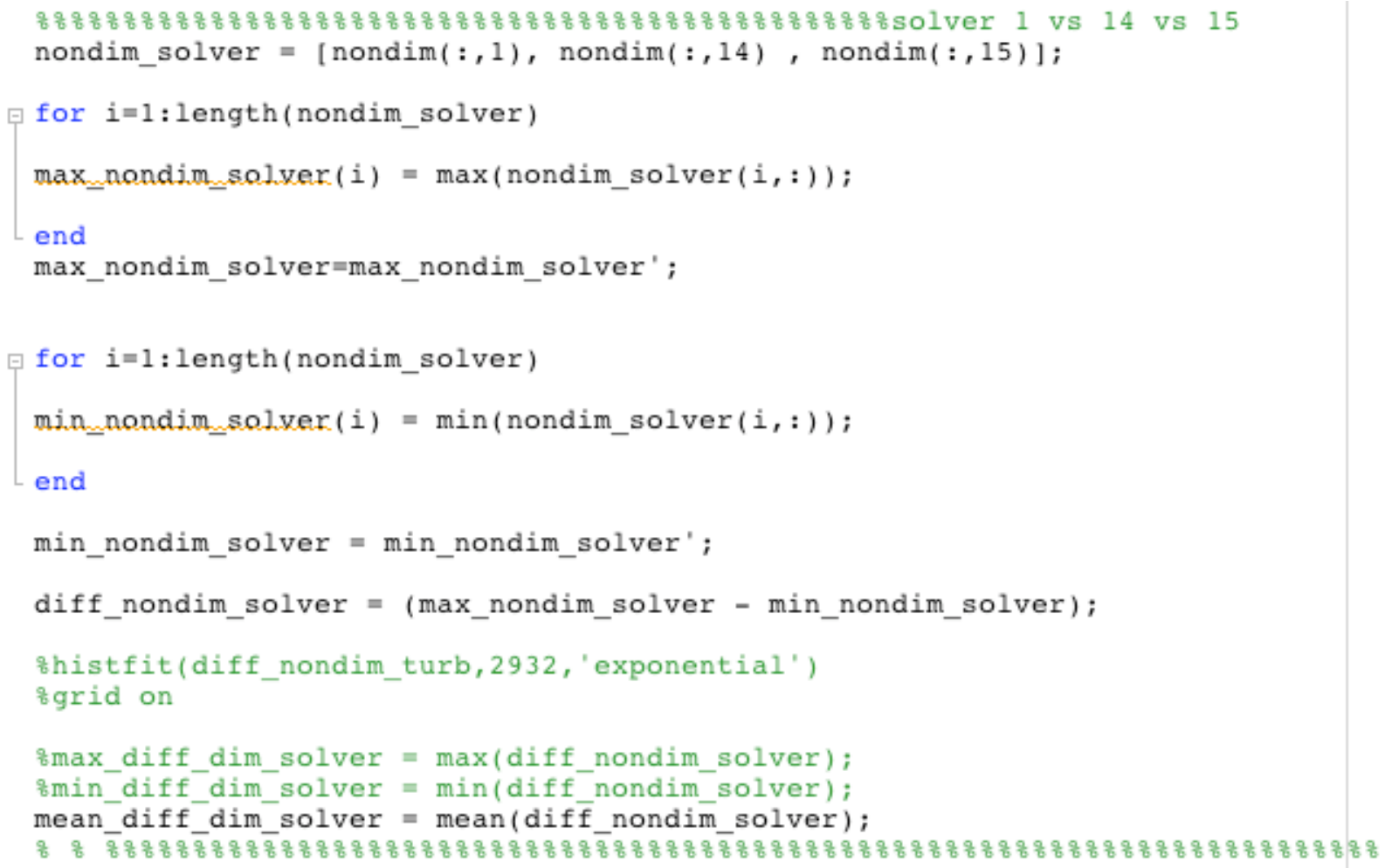




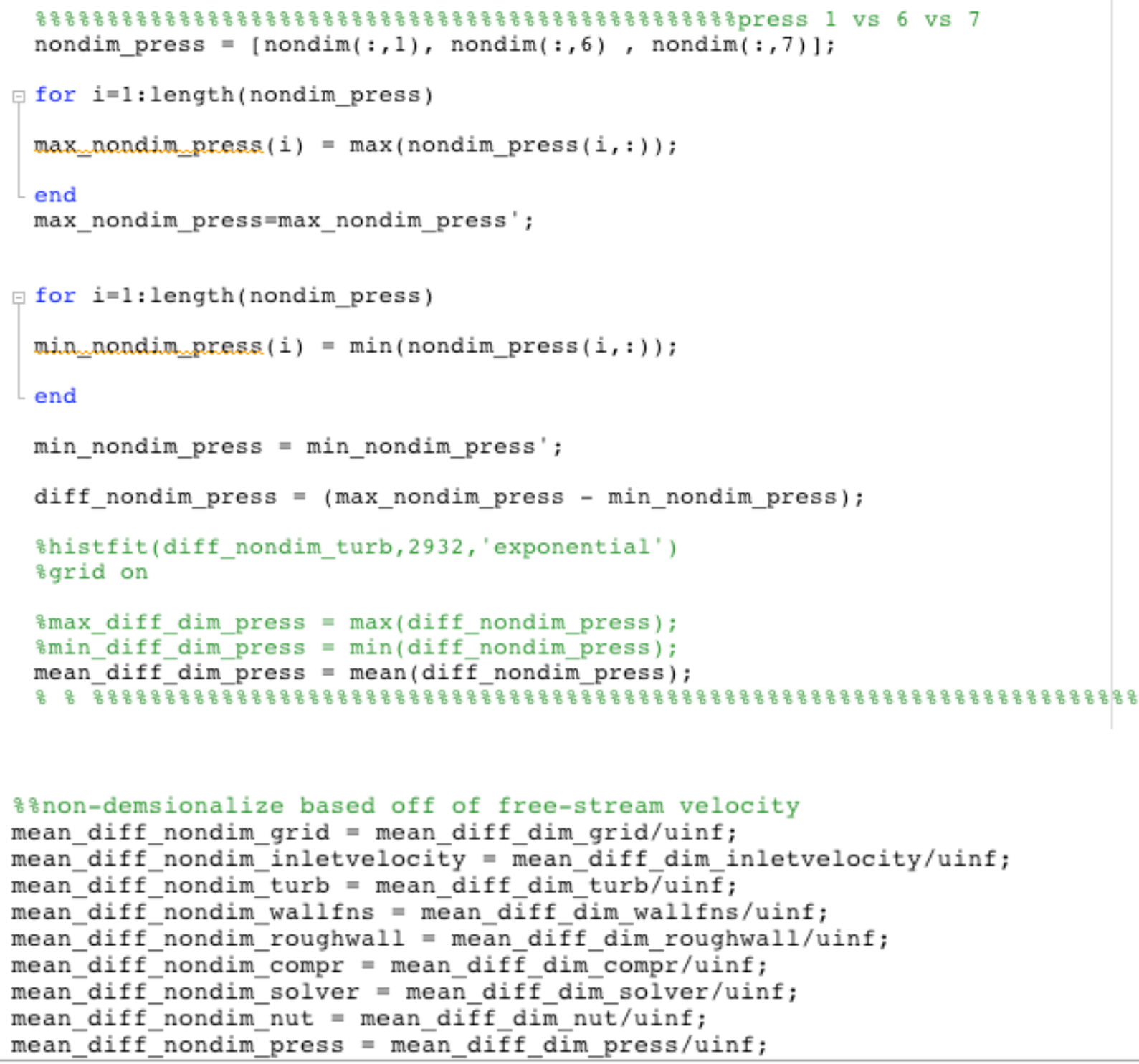


APPENDIX E: FLAT PLATE UNCERTAINTY MATLAB (FLATPLAT.M) 


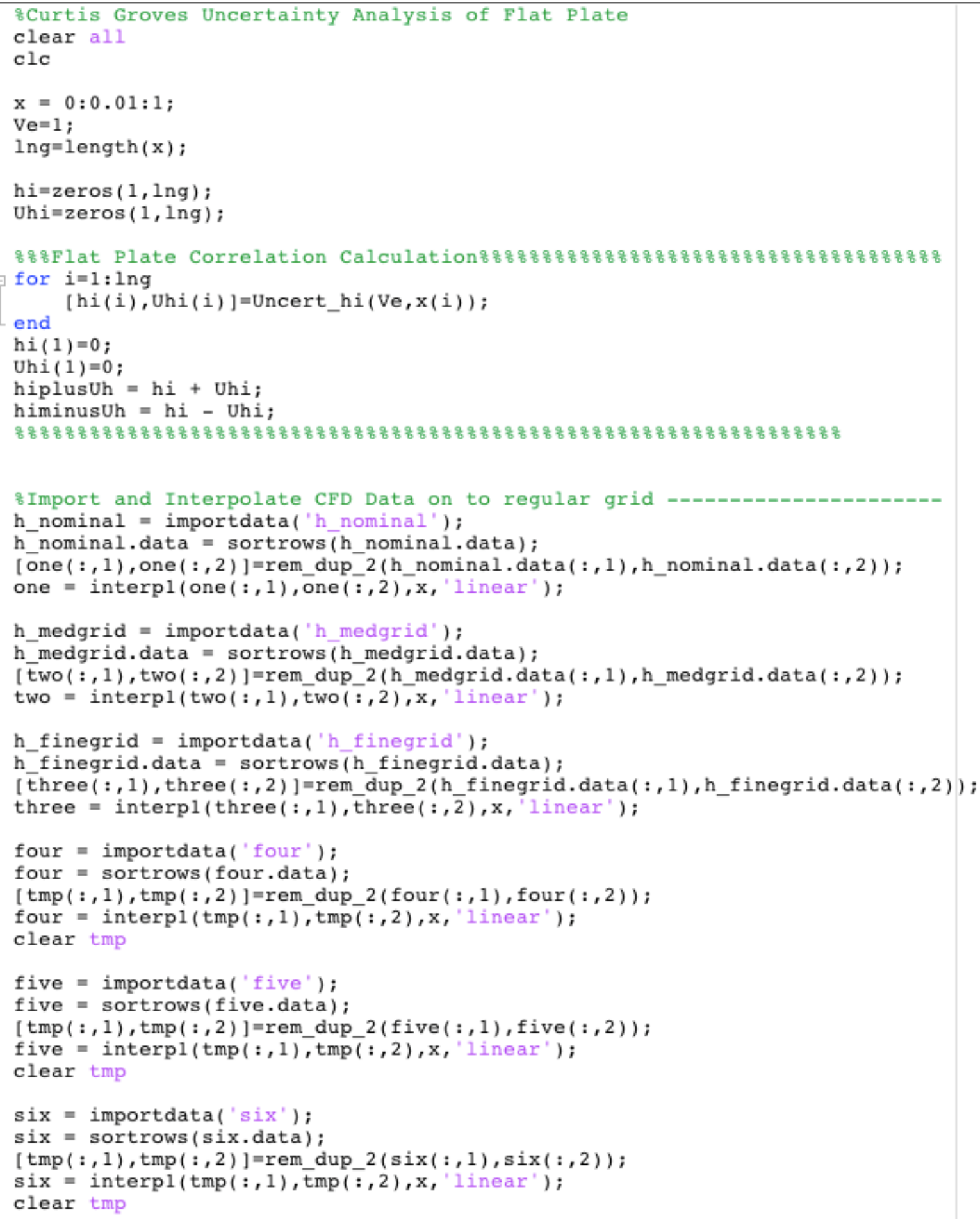




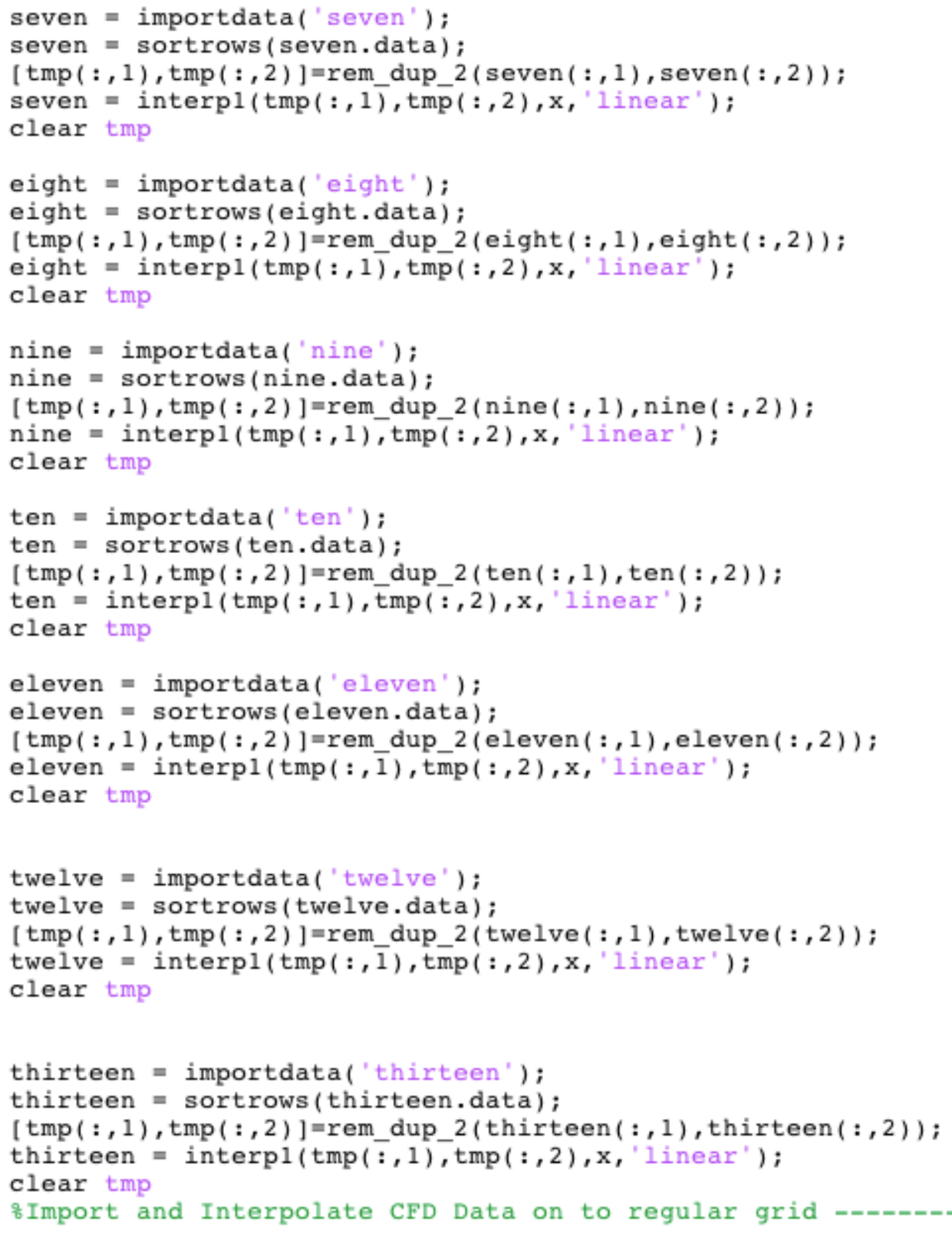




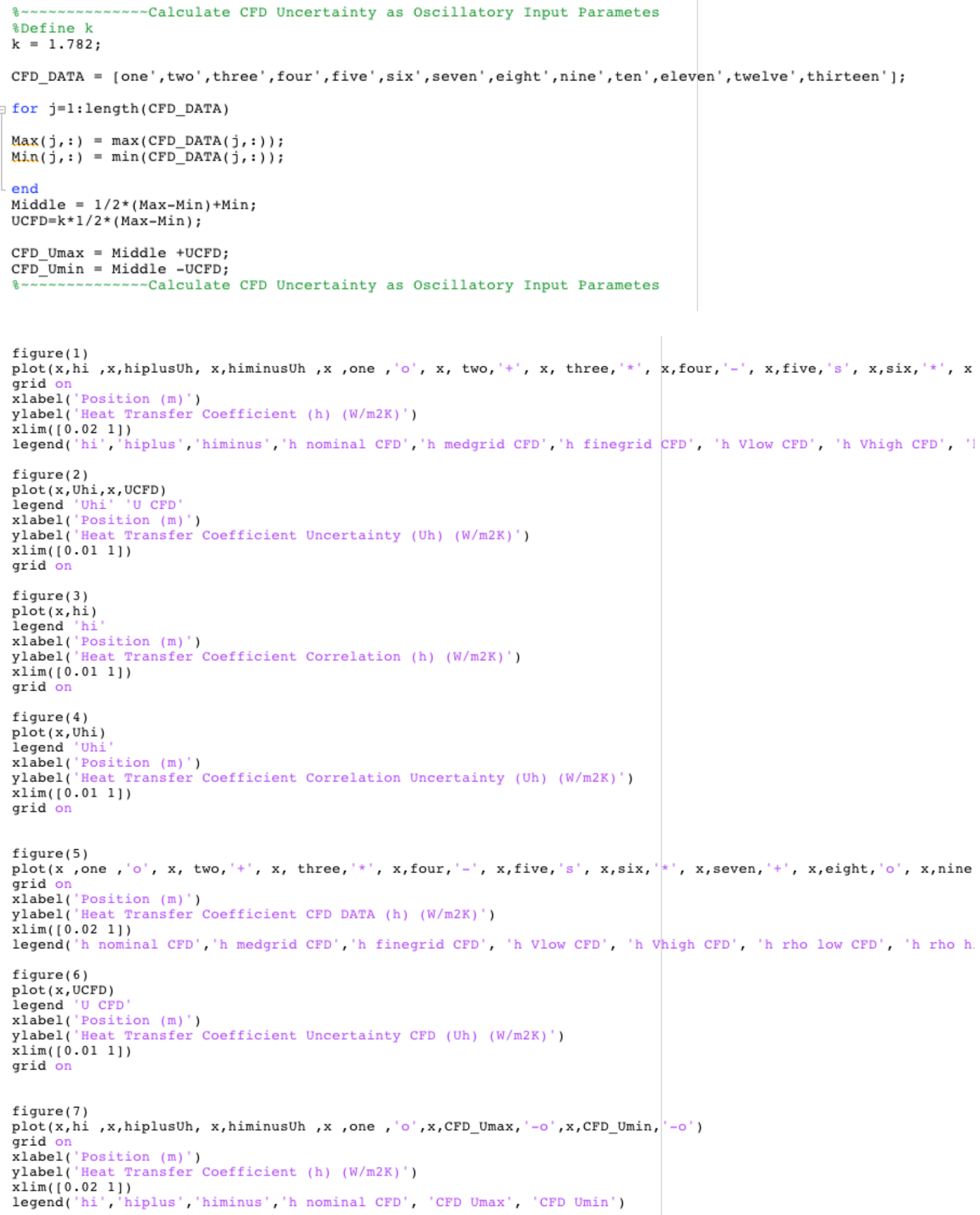


Appendix E.1 Heat Transfer Correlation Uncertainty Calculation (Uncert_Hi.M) 


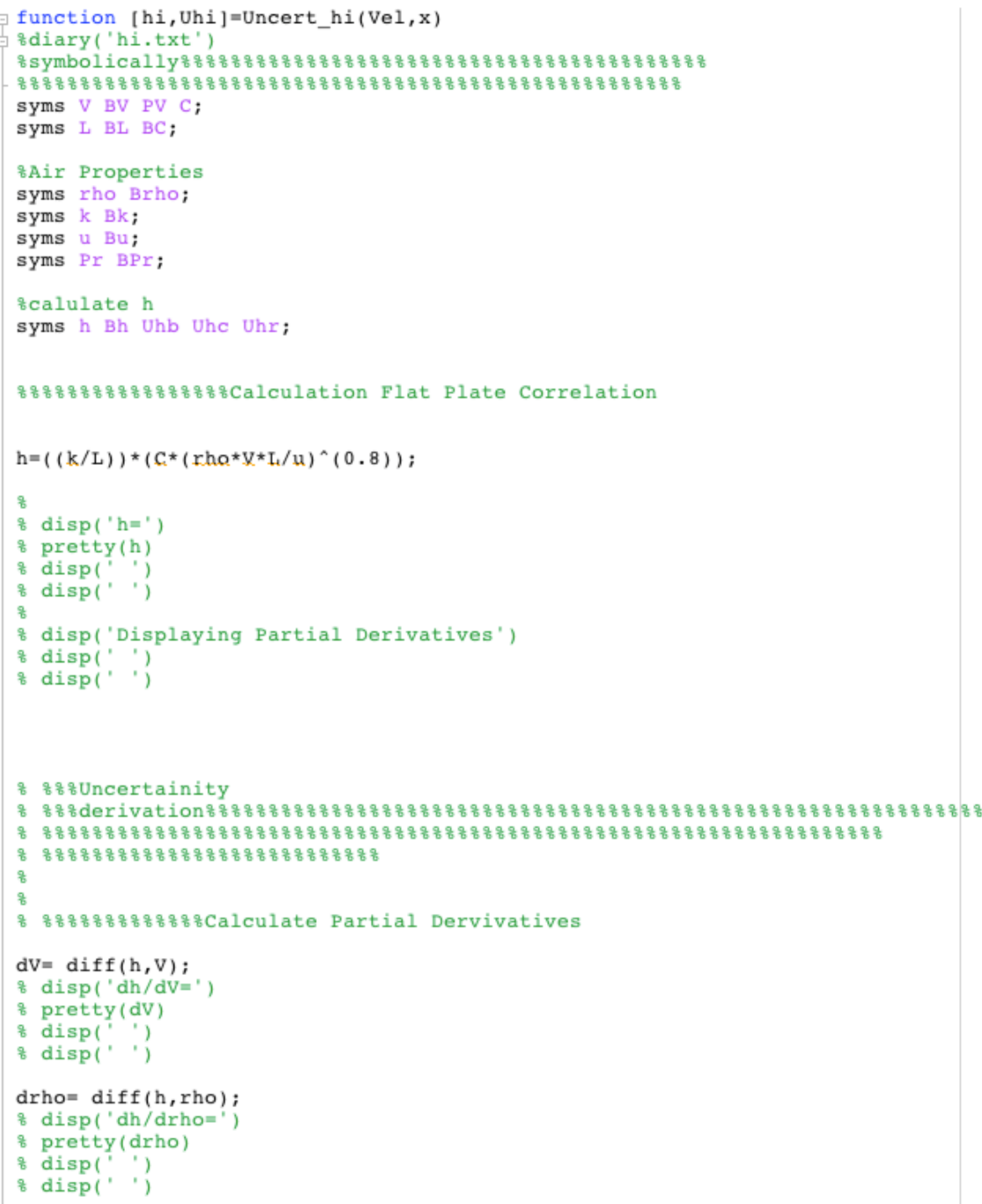




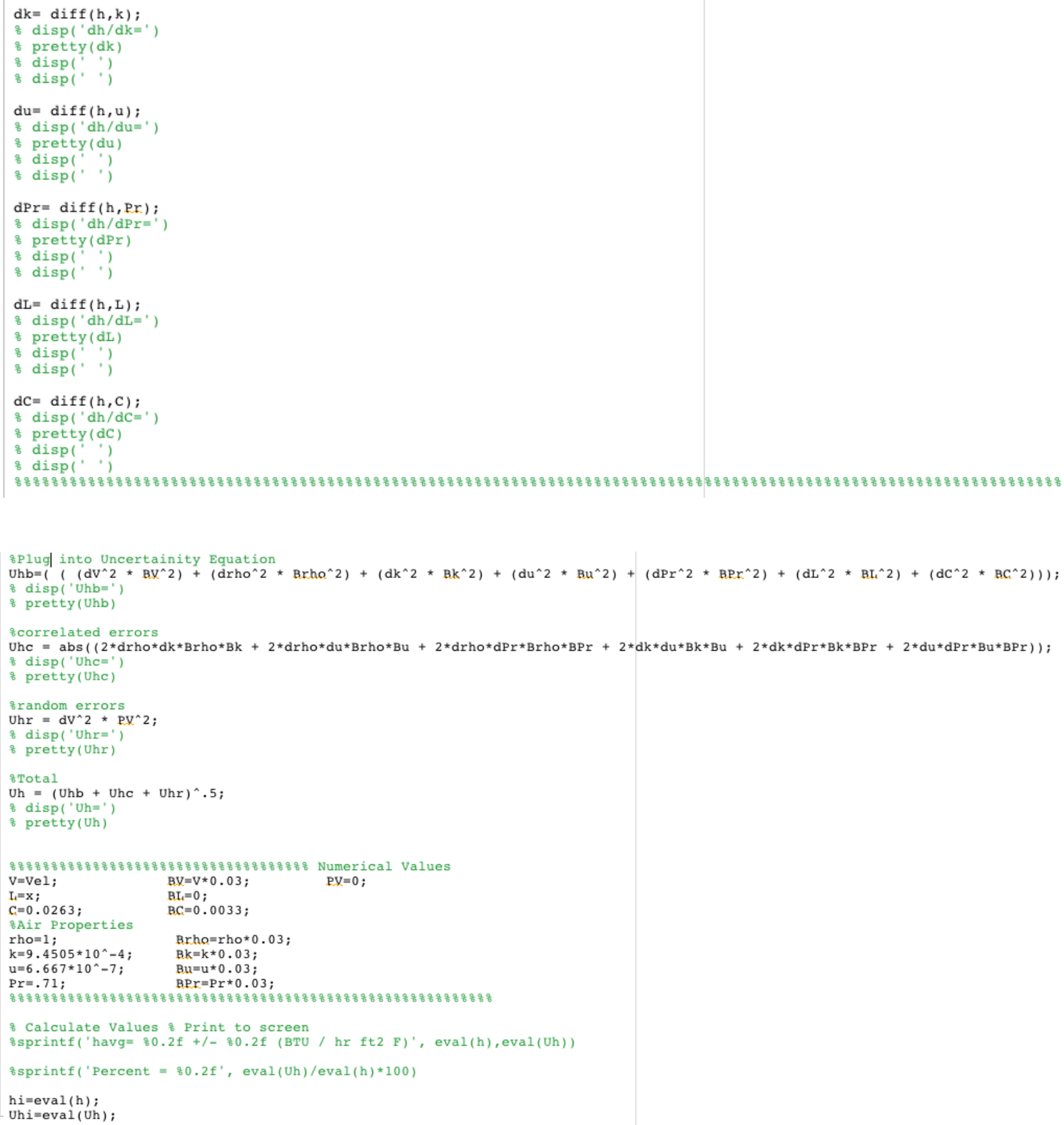


Appendix E.2 Remove Duplicates Script (Rem_Dup_2.M) 


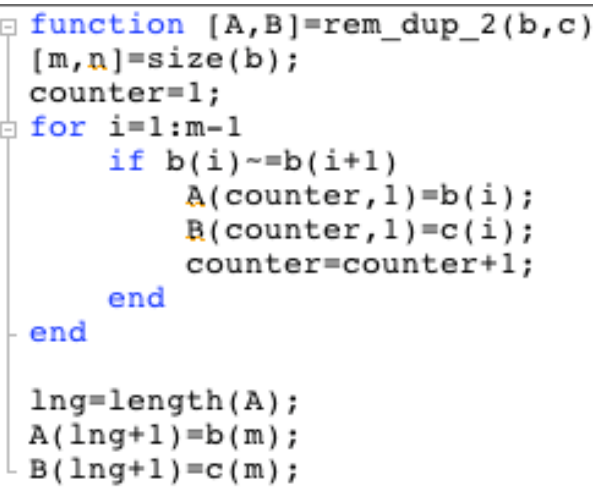




\section{APPENDIX F: SUMMARY OF AUTHORS PRIOR WORK AND}

BACKGROUND 


\section{Curtis E. Groves}

$\underline{\text { Research Interests: }}$

Computational Fluid Dynamics, Turbulence Modeling, Uncertainty Analysis, External Aerodynamics, Spacecraft Venting, Environmental Control Systems, Heat Transfer.

\section{$\underline{\text { Degrees: }}$}

- Ph.D., Mechanical Engineering, University of Central Florida, Expected Completion Date 2014.

- M.S., Aerospace Engineering, University of Central Florida, May 2012.

- B.S., Aerospace Engineering, West Virginia University, May 2008.

- B.S., Mechanical Engineering, West Virginia University, May 2008.

Recent Work:

- Mars Science Laboratory Mission, NASA, Environmental Control System Impingement Analysis

- Mars Science Laboratory Mission, NASA, Nuclear Launch Approval Processes

- NASA, Source Evaluation Board

- Tracking and Data Relay Satellites (TDRS K/L), NASA, Environmental Control System Impingement Analysis

- Tracking and Data Relay Satellites (TDRS K/L) Missions, NASA, Spacecraft Venting Analysis

- Interface Region Imaging Spectrograph (IRIS), NASA, Environmental Control System Impingement Analysis

- GLORY Mission, NASA, Environmental Control System Impingement Analysis, Testing, Uncertainty Analysis

$\underline{\text { Honors and Awards: }}$

- Group Achievement Award, ELVIS 2 Source Evaluation Board Team, NASA Kennedy Space Center, 2013.

- Group Achievement Award, Mars Science Lab, NASA Kennedy Space Center, 2012.

- Space Flight Awareness Award, NASA Kennedy Space Center, 2012.

- Certificate of Appreciation, NASA Kennedy Space Center, 2011.

- Distinguished Performance Rating, NASA, Launch Services Program, 2011.

- Completion of Accelerated Training Program, NASA Kennedy Space Center, 2008.

- NASA Cooperative Education Program, NASA Kennedy Space Center, 2006-2008.

- NASA West Virginia Space Grant Scholar, 2004-2008.

- Summa Cum Laude, West Virginia University, 2008. 
- Ralph. M Barnes Senior Scholastic Achievement Award, West Virginia University (Graduate with highest GPA during the junior and senior years), 2008.

- Promise Scholar, West Virginia University, 2003-2008.

- President's List, West Virginia University (4.0 GPA), 2003-2008.

$\underline{\text { Publications: }}$

1. Brink, J., Godfrey, G., Wittenborn, D., O’Keefe, D., Groves. C. (2005). OrbiterExternal Tank (ET) Mate Simulation. National Aeronautics and Space Administration: John F Kennedy Space Center 2005 Technology, Development and Application Annual Report. 129-130.

2. Godfrey, G., O’Keefe, D., Whittenborn, D., Groves, C., \& Kapr, F. (2006, December) Orbiter to External Fuel Tank Mating Simulation. Paper presented at Engineering Design Graphics Division Mid-Year Meeting in Ft. Lauderdale, Florida.

3. Groves, C., Ilie, M., Schallhorn, Paul. "Comprehensive Approach to Verification and Validation of CFD Simulations Applied to Backward Facing Step," AIAA-2013-0258, 2013.

4. Groves, C., Ilie, M., Schallhorn, Paul. "Computational Fluid Dynamics Uncertainty Analysis applied to Heat Transfer over a Flat Plate," American Physical Society Division of Fluid Dynamics, 2013. DFD13-2013-00087

5. Groves, C., Ilie, M., Schallhorn, Paul. "Computational Fluid Dynamics Uncertainty Analysis for Payload Fairing Spacecraft Environmental Control Systems," AIAA2014-0440, 2014.

6. Groves, C., Ilie, M., Schallhorn, Paul. "Interpolation Method needed for Numerical Uncertainty Analysis of Computational Fluid Dynamics," AIAA-2014-1433, 2014. 


\section{LIST OF REFERENCES}

[1] M. Kandula, K. Hammad and P. Schallhorn, "CFD Validation with LDV Test Data for Payload/Fairing Internal Flow," in AIAA-2005-4910, 2005.

[2] M. Kandula and L. Walls, "An application of overset grids to payload/fairing internal flow CFD analysis," in 20th AIAA Applied Aerodynamics Conference, AIAA-20023253, June 2002.

[3] P. J. Roache, K. N. Ghia and F. M. White, "Editorial Policy Statement on the Control of Numerical Accuracy," ASME Journal of Fluids Engineering, vol. 108, p. 2, 1986.

[4] J. Slater, "Uncertainty and Error in CFD Applications," NPARC Alliance CFD Verification and Validation, July 2008. [Online]. Available: http://www.grc.nasa.gov/WWW/wind/valid/tutorial/errors.html.

[5] F. Iudicello, "Guide for Verification and Validation of Comutational Fluid Dynamics Simulations," American Institute of Aeronautics and Astronautics (AIAA), no. AIAA G-077-1998, 1998.

[6] I. Celik and W. Zhang, "Calculation of Numerical Uncertainty Using Richardson Extrapolation: Application to Some Simple Turbulent Flow Calculations," American Society of Mechanical Engineers Journal of Fluid Mechanics, 1995. 
[7] P. J. Roache, Verification and Validation in Computational Science and Engineering, Hermosa Publishers, 1998.

[8] F. Stern, R. V. Wilson, H. W. Coleman and E. G. Patterson, "Verification and Validation of CFD Simulations," Iowa Institute of Hydraulic Research No.407, Septermber 1999.

[9] F. Stern, R. V. Wilson, H. W. Coleman and E. G. Patterson, "Comprehensive Approach to Verification anD Validation of CFD Simulations - Part 1: Methodology and Procedures," ASME Journal of Fluids Engineering, no. 123, pp. 793-802, 2001.

[10] F. Stern, R. V. Wilson, H. W. Coleman and E. G. Patterson, "Comprehensive Approach to Verification and Validation of CFD Simulations - Part 2: Application for Rans Simulation of a Cargo/Container Ship," ASME Journal of Fluids Engineering, vol. 123, pp. 803-810, 2001.

[11] "Uncertainty Analysis in CFD Verification and Validation Methodology and Procedures," in International Towing Tank Conference - Recommended Procedures and Guidelines, September 2008.

[12] I. Celik, U. Ghia, P. Roache, C. Freitas, H. Coleman and P. Raad, "“'Procedure for Estimating and Reporting of Uncertainity Due to Discretization in CFD Applications," American Society of Mechanical Enigneers Journal of Fluids Engineering, vol. 130, 2008. 
[13] I. Celik, Critical Issues With Quantification of Discretization Uncertainty in CFD, National Energy Technology Laboratory Workshop on Multiphase Flow Science, August 2011.

[14] "Standard for Verification and Validation in Computational Fluid Dynamics and Heat Transfer," The American Society of Mechanical Engineers, vol. An American National Standard, no. ASME V\&V 20-2009, 2009.

[15] C. Roy, "Review of Discretization Error Estimators in Scientific Computing," American Institute of Aeronautics and Astronautics, Vols. AIAA 2010-126.

[16] P. J. Roache, "Perspective: A Method for Uniform Reporting of Grid Refinement Studies," Journal of Fluids Engineering, vol. 116, pp. 405-413, 1994.

[17] L. F. Richardson, "'The Approximate Arithmetical Solution by Finite Differences of Physical Problems involving Differential Equations, with an Application to the Stresses in a Masonry Dam," Philosophical Transactions of the Royal Society of London. Series A, Containing Papers of a Mathematical or Physical Character, vol. 210, pp. 307-357, 1911.

[18] L. F. Richardson, "The Deferred Approach to the Limit, PartI. Single Lattice," Philosophical Transactions of the Royal Society of London. Series A, Containing Papers of a Mathematical or Physical Character, vol. 226, pp. 299-349, 1927. 
[19] C. Groves, M. Ilie and P. Schallhorn, "“'Comprehensive Approach to Verification and Validation of CFD Simulations Applied to Backward Facing Step," in AIAA-20130258, 2013.

[20] H. W. Coleman and W. G. Steele, Experimentation and Uncertainty Analysis for Engineers, 2nd Ed, New York: John Wiley \& Sons, Inc, 1999.

[21] C. E. Groves, P. A. Schallhorn and M. Ilie, "Computational Fluid Dynamics Uncertainty Analysis for Payload Fairing Spacecraft Enviornmental Control Systems," in AIAA-2014-0440, 2014.

[22] M. Kandula, R. Nallasamy, P. Schallhorn and L. Duncil, "An Application of Overset Grids to Payload / Fairing Three-Dimensional Internal Flow CFD Analysis," KSC-2007215, 2007.

[23] M. Kandula, R. Nallasamy, P. Schallhorn and L. Duncil, "'An Application of Overset Grids to Payload/Fairing Three-Dimensional Internal Flow CFD Analysis," Engineering Applications of Computational Fluid Mechanics, vol. 2, no. 1, pp. 119-129, 2008.

[24] M. Kandula, K. Hammad and P. Schallhorn, "CFD Validation with LDV Test Data for Payload/Fairing Internal Flow," KSC-2005-4910, 2005. 
[25] "Launching Rockets," NASA, [Online]. Available:

http://www.nasa.gov/centers/kennedy/launchingrockets/. [Accessed 28 January 2013].

[26] "Delta II Payload Planners Guide," United Launch Alliance, [Online]. Available: http://www.ulalaunch.com/site/docs/product_cards/guides/DeltaIIPayloadPlanners Guide2007.pdf. [Accessed 28 January 2013].

[27] "Delta IV Payload Planners Guide," [Online]. Available: http://www.ulalaunch.com/site/docs/product_cards/guides/DeltaIVPayloadPlanners Guide2007.pdf. [Accessed 28 January 2013].

[28] "Atlas 5 Payload Planners Guide," [Online]. Available: http://www.ulalaunch.com/site/docs/product_cards/guides/AtlasVUsersGuide2010. pdf. [Accessed 28 January 2013].

[29] "Pegasus Users Guide.," Orbital Sciences Coorporation, [Online]. Available: http://www.orbital.com/NewsInfo/Publications/Pegasus_UG.pdf. [Accessed 28 January 2013].

[30] "Taurus Users Guide.," [Online]. Available: http://www.orbital.com/NewsInfo/Publications/taurus-user-guide.pdf. [Accessed 28 January 2013]. 
[31] "Space X Falcon 9 Users Guide," Space X, [Online]. Available:

http://www.spacex.com/Falcon9UsersGuide_2009.pdf. [Accessed 28 January 2013].

[32] C. E. Groves, P. A. Schallhorn and M. Ilie, "Interpolation Method needed for Numerical Uncertainty Analysis of Computational Fluid Dynamics," in AIAA-2014-1433, 2014.

[33] I. Celik and O. Karatekin,, "Numerical Experiments on Application of Richardson Extrapolation With Nonuniform Grids," ASME Journal of Fluids Engineering, vol. 119, no. 3, p. 584, 1997.

[34] ANSYS FLUENT 12.0 User's Guide.

[35] "OpenFOAM User Guide," The OpenFOAM Foundation, [Online]. Available: http://www.openfoam.org/docs/user/.

[36] "Matlab," Mathworks Product Website, [Online]. Available: http://www.mathworks.com/products/matlab.

[37] R. Fox and A. McDonald, Introduction to Fluid Mechanics, 3rd Edition, John Wiley \& Sons, 1985.

[38] P. L. Roache and P. M. Knupp, "Completed Richardson Extrapolation," Communications in Numerical Methods in Engineering, vol. 9, pp. 365-374, 1993. 
[39] J. S. Brock, "Bounded Numerical Error Estimates for Oscillatory Convergence of Simulation Data," in AIAA-2007-4091, 2007.

[40] I. Celik, J. Li, G. Hu and C. Shaffer, "Limitations of Richardson's Extrapolation and Some Possible Remedies," in 2004 ASME Heat Transfer/Fluids Engineering Summer Conference, Charlotte, North Carolina, 2004.

[41] H. W. Coleman, F. Stern, A. Di Mascio and E. Campana, "The Problem with Oscillatory Behavior in Grid Convergence Studies," ASME Journal of Fluids Engineering, vol. 123, pp. 438-439, 2001.

[42] L. Eca and M. Hoekstra, "An Evaluation of Verification Procedures for CFD Applications," in 24th Symposium on Naval Hydrodynamics, Fukuoka, Japan, 2002.

[43] C. E. Groves, P. A. Schallhorn and M. Ilie, "Computational Fluid Dynamics Uncertainty Analysis applied to Heat Transfer over a Flat Plate," in American Physical Society Division of Fluid Dynamics DFD13-2013-00087, 2013.

[44] "FLUENT - Forced Convection over a Flat Plate," Cornell University, [Online]. Available: https://confluence.cornell.edu/display/SIMULATION/FLUENT++Forced+Convection+over+a+Flat+Plate. 